\title{
Double Thiol-Chiral Brønsted Base Catalysis: Asymmetric Cross Rauhut-Currier Reaction and Sequential [4 + 2] Annulation for Assembly of Different Activated Olefins
}

Zhi Zhou, ${ }^{\dagger}{ }^{\S}$ Qing He ${ }^{\dagger, \S}$ Ying Jiang, ${ }^{\dagger}$ Qin Ouyang, ${ }^{\dagger}$ Wei Du, ${ }^{* \dagger}$ and Ying-Chun Chen $*^{\dagger, \ddagger}$

${ }^{\dagger}$ Key Laboratory of Drug-Targeting and Drug Delivery System of the Ministry of Education and Sichuan Research Center for Drug Precision Industrial Technology, West China School of Pharmacy, Sichuan University, Chengdu 610041, China ¥College of Pharmacy, Third Military Medical University, Chongqing 400038, China

e-mail: duweiyb@scu.edu.cn; ycchen@scu.edu.cn

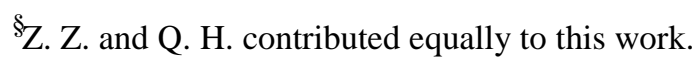

\section{Supporting Information}

1. General methods S2

2. More screening conditions for the cross $\mathrm{RC}$ reaction of enone 1a with isatin-derived alkylidene malononitrile $2 \mathrm{a}$. . .52

3. Screening conditions for the three-component domino $[2+2+2]$ annulation and substrate scope.

4. More screening conditions for the cross RC reaction of 2-cyclohexenone with 2a and onepot $[4+2]$ annulation. . .56

5. General procedure for the cross $\mathrm{RC}$ reactions of enones 1 with isatin-derived alkylidene malononitriles 2 and annulations S7

6. More screening conditions for the cross $\mathrm{RC}$ reaction of enone 1a with $\alpha$-cyano chalcone 11a and annulation. S21

7. General procedure for the cross RC reactions of enone 1a with $\alpha$-cyano chalcones 11 and annulations S22

8. Reaction at a $1.0 \mathrm{mmol}$ scale S29

9. Procedure for the cross $\mathrm{RC}$ reaction-initiated [4 + 2] annulation .S30

10. More attempts for the cross $\mathrm{RC}$ reaction-initiated $[4+2]$ annulations S30

11. Synthetic transfomations of the annulation products $5 \mathrm{a}$ and 12a S31

12. Crystal data and structure refinement for enantiopure $5 \mathrm{~g}$ and $12 \mathrm{f}$ S35

13. DFT calculations of the key intermediates for the asymmetric cross Rauhut-Currier reaction and proposed catalytic mechanism .S38

14. NMR spectra and HPLC chromatograms S57 


\section{General methods}

NMR data were obtained for ${ }^{1} \mathrm{H}$ at $400 \mathrm{MHz}$ or $600 \mathrm{MHz}$, and for ${ }^{13} \mathrm{C}$ at $100 \mathrm{MHz}$ or $150 \mathrm{MHz}$. Chemical shifts were reported in ppm from tetramethylsilane with the solvent resonance as the internal standard in $\mathrm{CDCl}_{3}$ solution. ESI HRMS was recorded on a Waters SYNAPT G2. In each case, enantiomeric ratio was determined by HPLC analysis on a chiral column in comparison with the authentic racemate, using a Daicel Chiralpak AD-H Column $(250 \times 4.6 \mathrm{~mm})$, Chiralcel OD-H Column $(250 \times 4.6 \mathrm{~mm})$, Chiralpak IB Column $(250 \times 4.6 \mathrm{~mm})$, Chiralpak ID Column $(250 \times 4.6$ $\mathrm{mm}$ ) UV detection was monitored at $220 \mathrm{~nm}$ or $254 \mathrm{~nm}$. Optical rotation was measured in $\mathrm{CHCl}_{3}$ solution at $25^{\circ} \mathrm{C}$ or $20^{\circ} \mathrm{C}$. Column chromatography was performed on silica gel (200-300 mesh) eluting with EtOAc and petroleum ether. TLC was performed on glass-backed silica plates. UV light, $\mathrm{I}_{2}$, and solution of potassium permanganate were used to visualize products or starting materials. All chemicals were used without purification as commercially available unless otherwise noted. Petroleum ether and EtOAc were distilled. THF was freshly distilled from sodium/benzophenone before use. Experiments involving moisture and/or air sensitive components were performed under a positive pressure of argon in oven-dried glassware equipped with a rubber septum inlet. Dried solvents and liquid reagents were transferred by oven-dried syringes. The substrates were synthesized according to the literature procedures. ${ }^{1}$

(1) (a) Peng, J.; Ran, G.-Y.; Du, W.; Chen, Y.-C. Org. Lett. 2015, 17, 4490-4493. (b) Shi, M.-L.; Zhan, G.; Zhou, S.-L.; Du, W.; Chen, Y.-C. Org. Lett. 2016, 18, 6480-6483.

\section{More screening conditions for the cross $\mathrm{RC}$ reaction of enone 1a with isatin- derived alkylidene malononitrile $2 \mathrm{a}$}

As noted in the text, the reaction was performed with 2-mercaptobenzoic acid T1, cinchonidine derived PTC and $\mathrm{K}_{2} \mathrm{CO}_{3}$ in toluene at $25{ }^{\circ} \mathrm{C}$ for $24 \mathrm{~h}$ to $48 \mathrm{~h}$, giving the RC adduct with 83:17 er, $78 \%$ yield. Then an array of PTCs were investigated, and the cinchonine derived PTC C2 gave the best enantioselectivity and reactivity (92\% yield, 93:7 er). 


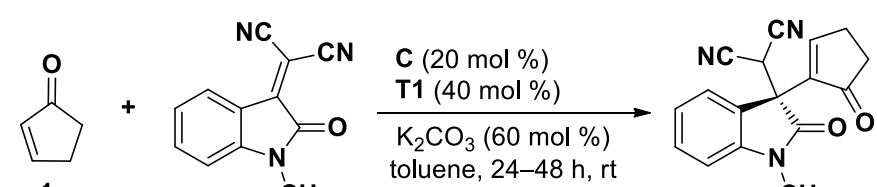

$1 \mathrm{a}$

2a $\stackrel{\mathrm{C}}{\mathrm{C}} \mathrm{H}_{3}$

${ }_{3 a} \mathrm{CH}_{3}$

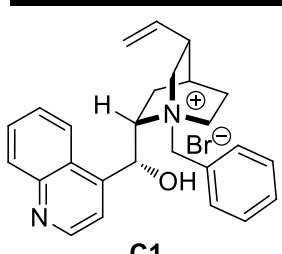

C1

$83: 17$ er, $78 \%$ yield

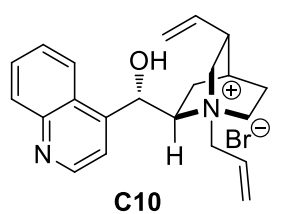

$72: 28$ er, $65 \%$ yield

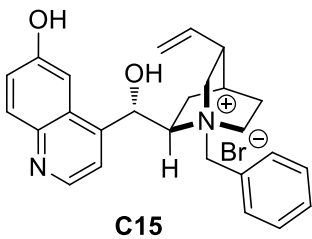

$65: 35$ er, $45 \%$ yield

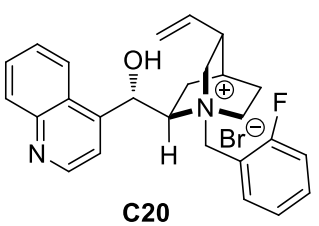

$60: 40$ er, $60 \%$ yield

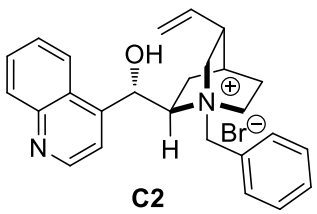

93:7 er, $92 \%$ yield

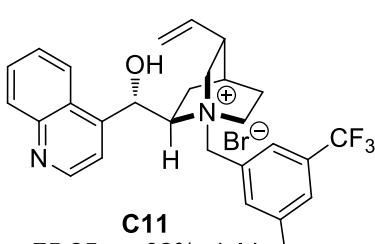

$75: 25$ er, $62 \%$ yield $\mathrm{CF}_{3}$

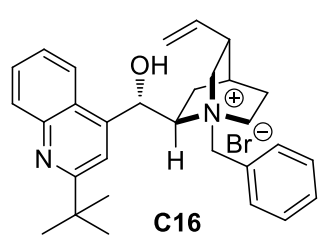

$68: 32$ er, $72 \%$ yield

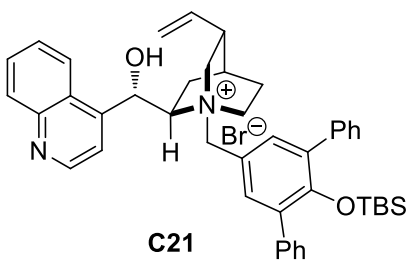

$58: 42$ er, $65 \%$ yield

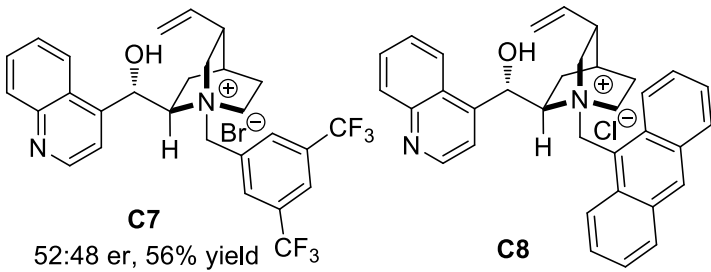

$30: 70$ er, $88 \%$ yield

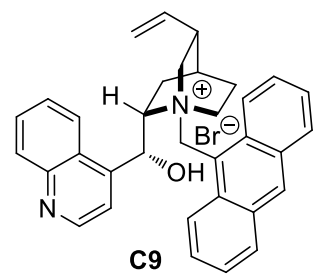

$68: 32$ er, $85 \%$ yield

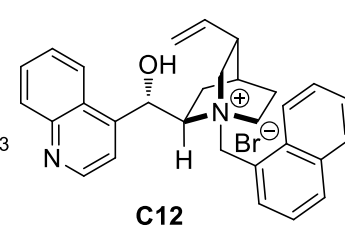

$78: 22$ er, $76 \%$ yield

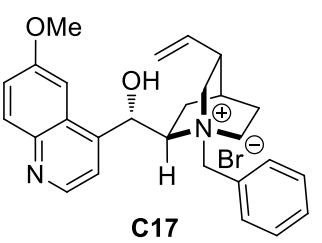

$67: 33$ er, $68 \%$ yield

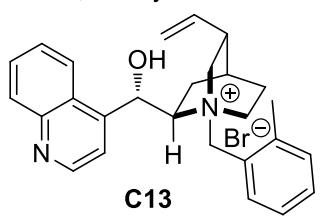

$75: 25$ er, $53 \%$ yield

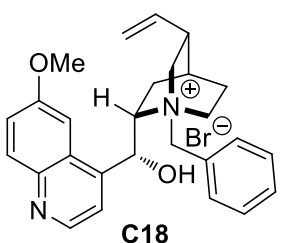

$34: 66$ er, $75 \%$ yield

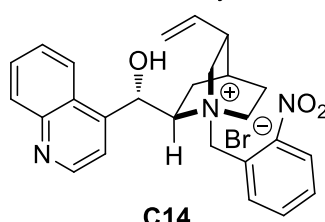

$55: 45 \%$ er, $67 \%$ yield

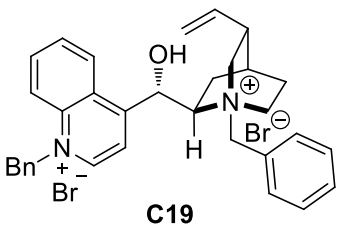

N.R.

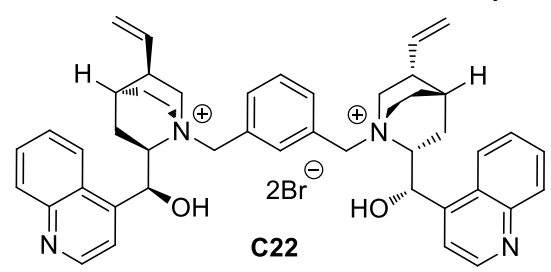

$51: 49$ er, $62 \%$ yield

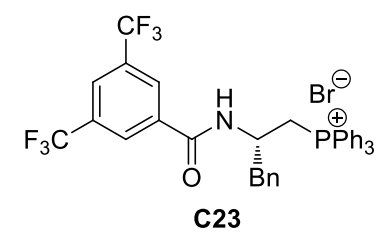

$54: 46$ er, $66 \%$ yield

After screening many parameters, we paid our attention to structural modifications of the catalysts.

Some bifunctional PTCs were prepared and investigated.

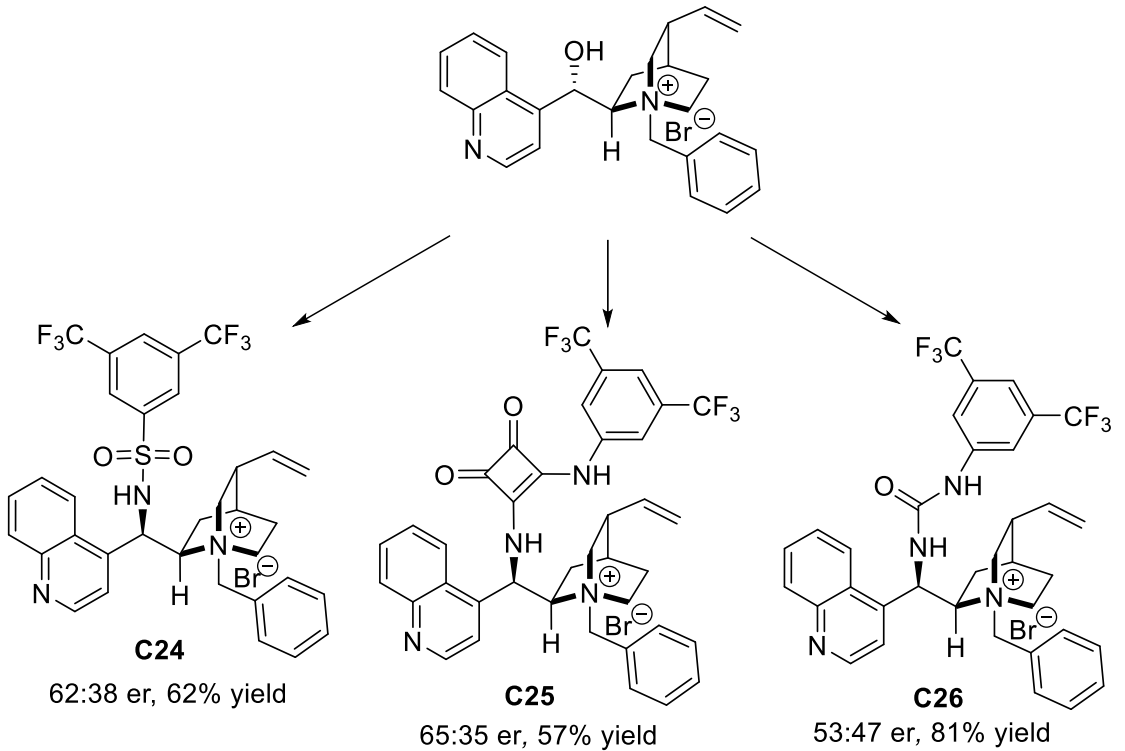


More screening studies on other electrophiles resulted in no success.<smiles>O=C1C=CC[CH+]1</smiles>

$1 \mathrm{a}$<smiles>N#CC(C#N)=Cc1ccccc1</smiles>

N.R.

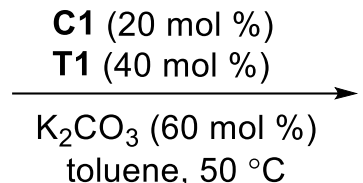

toluene, $50{ }^{\circ} \mathrm{C}$<smiles>[R]CC([R])C1=CCCC1=O</smiles>

$\mathrm{R}$<smiles>C1CCCCC1</smiles><smiles>O=C1C(=Cc2ccccc2)C(=O)c2ccccc21</smiles>

N.R.

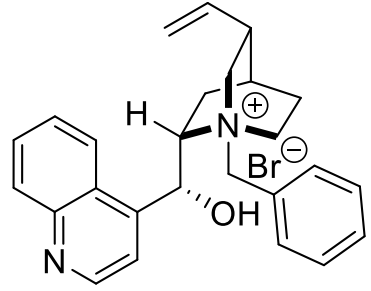

C1<smiles>O=C(/C=C/c1ccc(Br)cc1)c1ccccc1</smiles>

N.R.

\section{Screening conditions for the three-component domino $[2+2+2]$ annulation}

\section{and substrate scope}

3.1 Screening conditions for the three-component domino $[2+2+2]$ annulation $^{a}$

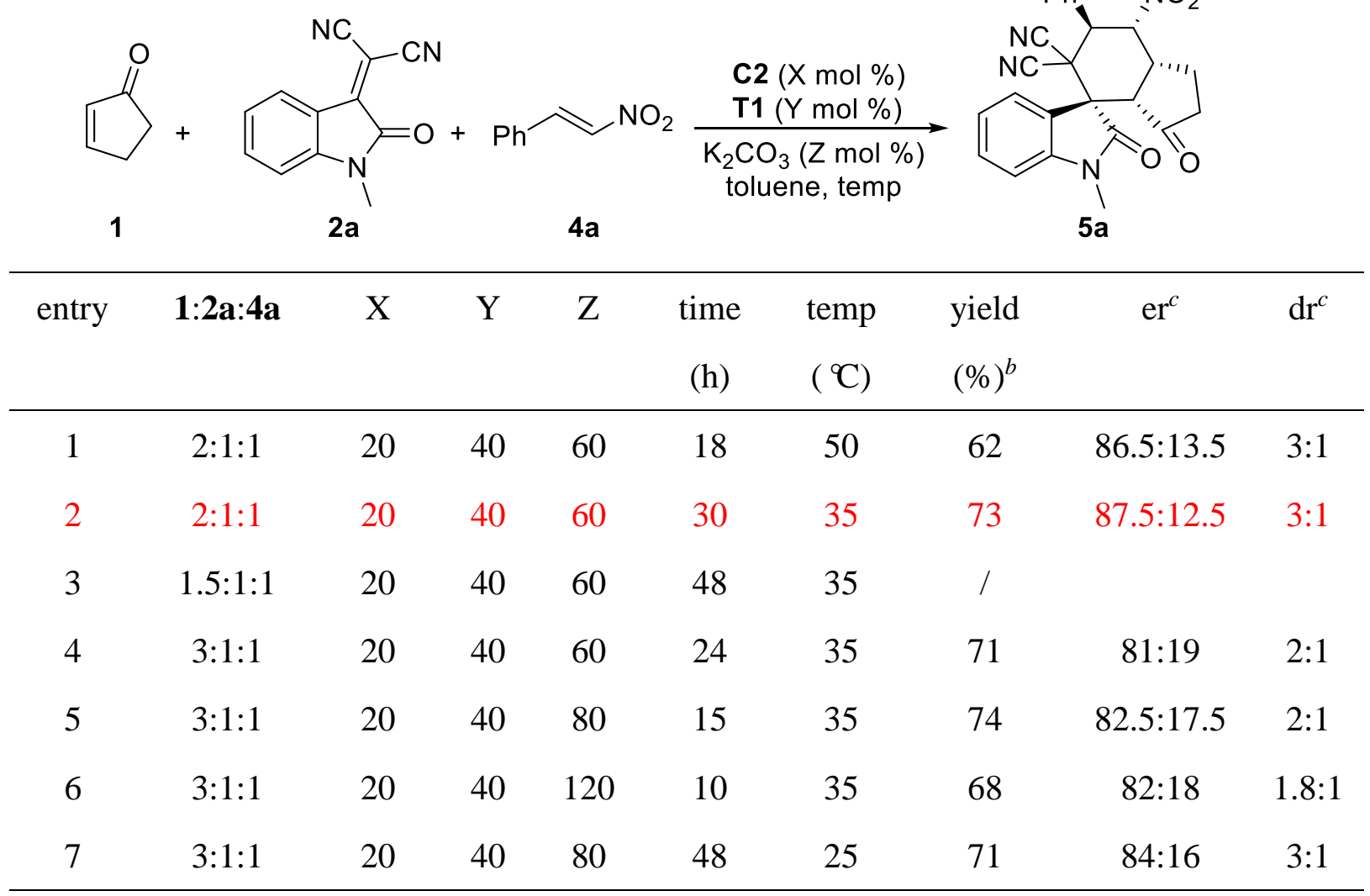

${ }^{a}$ Unless noted otherwise, reactions were performed with 2-cyclopentenone 1a $(0.2 \mathrm{mmol})$, activated olefin $2 \mathbf{a}(0.1 \mathrm{mmol})$, nitroolefin $4 \mathbf{a}(0.1 \mathrm{mmol}), \mathrm{PTC} \mathbf{C 2}$, thiol T1 and $\mathrm{K}_{2} \mathrm{CO}_{3}$ in toluene $(1.0 \mathrm{~mL})$ for $10-48 \mathrm{~h} .{ }^{b}$ Isolated yield. ${ }^{c}$ Determined by HPLC analysis on a chiral stationary phase. 
It was found that chemoselective assembly of the three different activated alkenes could be conducted under the catalysis of thiol T1 and PTC C2. However, the reaction would quickly become dark after the early addition of nitroolefin $\mathbf{4 a}$, which might probably result from its aggregation promoted by thiol addition. As summarized in the above table, the yield together with stereoselectivity generally decreased significantly compared to those in the sequential one-pot process, even under the optimized conditions.

3.2 Substrate scope of the three-component domino $[2+2+2]$ annulation $^{a}$

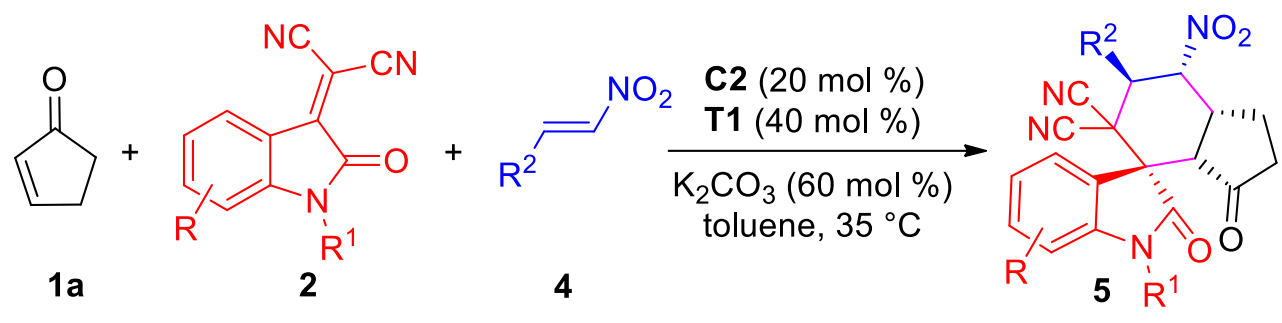

\begin{tabular}{|c|c|c|c|c|c|}
\hline entry & $\mathrm{R}, \mathrm{R}^{1}$ & $\mathrm{R}^{2}$ & yield $(\%)^{b}$ & $\mathrm{er}^{c}$ & $\mathrm{dr}^{c}$ \\
\hline 1 & $\mathrm{H}, \mathrm{Me}$ & $\mathrm{Ph}$ & 5a, 73 & $87.5: 12.5$ & $3: 1$ \\
\hline 2 & $\mathrm{H}, \mathrm{Bn}$ & $\mathrm{Ph}$ & $\mathbf{5 b}, 70$ & $82: 18$ & $3.2: 1$ \\
\hline 3 & $\mathrm{H}$, allyl & $\mathrm{Ph}$ & 5c, 63 & $82: 18$ & 4.5:1 \\
\hline 4 & $\mathrm{H}, \mathrm{MOM}$ & $\mathrm{Ph}$ & 5d, 68 & $80.5: 19.5$ & $3.3: 1$ \\
\hline 5 & 7-Me, Me & $\mathrm{Ph}$ & 5f, 62 & $80: 20$ & 2.5:1 \\
\hline 6 & 5-MeO, Me & $\mathrm{Ph}$ & 5g, 71 & $78.5: 21.5$ & $2: 1$ \\
\hline 7 & $5,7-\mathrm{Me}_{2}, \mathrm{Me}$ & $\mathrm{Ph}$ & 5h, 72 & $85.5: 14.5$ & $4: 1$ \\
\hline 8 & $5,7-\mathrm{Me}_{2}, \mathrm{Me}$ & $2-\mathrm{BrC}_{6} \mathrm{H}_{4}$ & 5o, 61 & $85: 15$ & $1: 1$ \\
\hline 9 & $5,7-\mathrm{Me}_{2}, \mathrm{Me}$ & 2-naphthyl & $5 r, 62$ & $87.5: 12.5$ & $5: 1$ \\
\hline 10 & $5,7-\mathrm{Me}_{2}, \mathrm{Me}$ & 2-thienyl & $\mathbf{5 s}, 59$ & $86.5: 13.5$ & $3: 1$ \\
\hline
\end{tabular}

${ }^{a}$ Unless noted otherwise, reactions were performed with 2-cyclopentenone 1a $(0.2 \mathrm{mmol})$, activated olefin 2 (0.1 mmol), nitroolefin 4 (0.1 mmol), PTC C2 $(20 \mathrm{~mol} \%)$, thiol T1 $(40 \mathrm{~mol} \%)$ and $\mathrm{K}_{2} \mathrm{CO}_{3}(60 \mathrm{~mol} \%)$ in toluene $(1.0 \mathrm{~mL})$ at $35{ }^{\circ} \mathrm{C}$ for $24-48 \mathrm{~h} .{ }^{b}$ Isolated yield. ${ }^{c}$ Determined by HPLC analysis on a chiral stationary phase.

Some other activated olefins or nitroolefins were applied to the three-component reaction as well, and the corresponding products $\mathbf{5}$ were generally obtained with moderate yields and stereoselectivity. Therefore, better data were obtained by conducting the $\mathrm{RC} /[4+2]$ annulation in a sequential process. 


\section{More screening conditions for the cross $\mathrm{RC}$ reaction of 2-cyclohexenone with}

\section{2a and one-pot $[4+2]$ annulation $^{a}$}

(12)

${ }^{a}$ Unless noted otherwise, reactions were performed with 2-cyclohexenone $(0.8 \mathrm{mmol})$, activated alkene $\mathbf{2}(0.1 \mathrm{mmol})$, PTC C2 $(20 \mathrm{~mol} \%)$, thiol T (40 mol \%), base $(60 \mathrm{~mol} \%)$ in toluene $(0.5 \mathrm{~mL})$ at $30^{\circ} \mathrm{C}$ for $48-72 \mathrm{~h}$. After completion, nitroolefin $4(0.1 \mathrm{mmol})$ was added, and stirred at $50{ }^{\circ} \mathrm{C}$ for $4 \mathrm{~h} .{ }^{b}$ Isolated yield. ${ }^{c}$ Determined by HPLC analysis on a chiral stationary phase; $\mathrm{dr}>19: 1$.

As outlined in the above table, we further screened a number of thiol compounds for the reaction of 2-cyclohexenone, but the results could not be further improved. 


\section{General procedure for the cross RC reactions of enones 1 with isatin-derived}

\section{alkylidene malononitriles 2 and annulations}

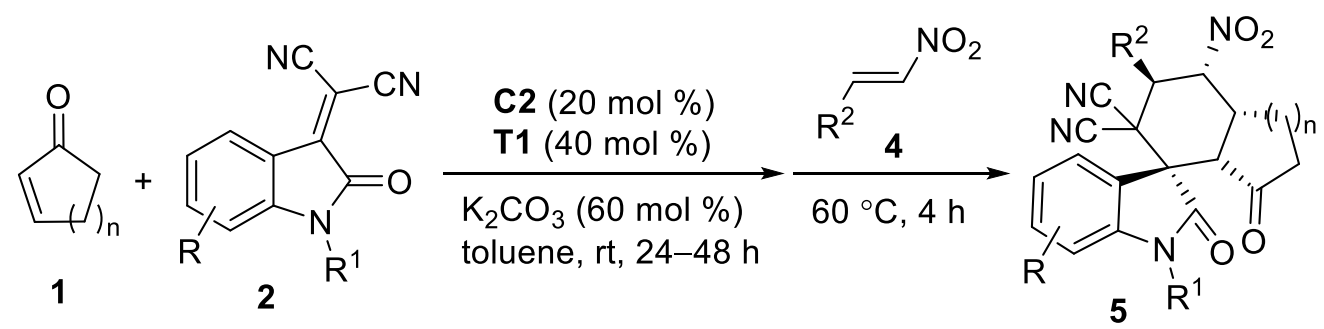

A mixture of cyclic enone 1 ( $0.2 \mathrm{mmol}, 2.0$ equiv), activated olefin 2 ( $0.1 \mathrm{mmol}, 1.0$ equiv), PTC C2 $(20 \mathrm{~mol} \%)$, thiol T1 $(40 \mathrm{~mol} \%)$ and $\mathrm{K}_{2} \mathrm{CO}_{3}(60 \mathrm{~mol} \%)$ in toluene $(1.0 \mathrm{~mL})$ was stirred at room temperature for $24 \mathrm{~h}$. After completion, nitroolefin 4 ( $0.1 \mathrm{mmol}, 1.0$ equiv) was added and the reaction was stirred at $60{ }^{\circ} \mathrm{C}$ for $4 \mathrm{~h}$. Then the annulation product 5 was obtained by flash chromatography on silica gel $($ EtOAc/petroleum ether $=1 / 8-1 / 5)$.

Most racemates could be obtained by using triethylamine and thiol T1 as the catalysts, but a few reactions failed. So two peaks of these enantiomers were assigned by HPLC analysis on a chiral stationary phase with the mixture of two enantiomers, which were produced by using quinine and quindine as the catalyst, respectively.

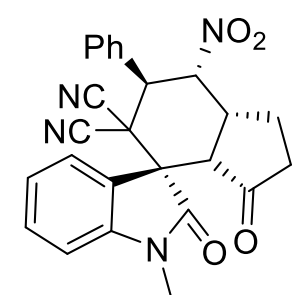

Synthesis of 5a: 2-Cyclopentenone 1a (16.4 mg, $0.2 \mathrm{mmol}), 2-(1-\mathrm{methyl}-2-$ oxoindolin-3-ylidene)malononitrile $2 \mathbf{a}(20.9 \mathrm{mg}, 0.1 \mathrm{mmol})$, PTC C2 (9.3 mg, $0.02 \mathrm{mmol}), 2$-mercaptobenzoic acid T1 (6.2 mg, $0.04 \mathrm{mmol})$ and $\mathrm{K}_{2} \mathrm{CO}_{3}(8.3 \mathrm{mg}$, $0.06 \mathrm{mmol})$ were stirred in distilled toluene $(1.0 \mathrm{~mL})$ at $\mathrm{rt}$ for $24 \mathrm{~h}$. Then $(E)-\beta$ nitrostyrene 4a $(14.9 \mathrm{mg}, 0.1 \mathrm{mmol})$ was added and the reaction was stirred at $60{ }^{\circ} \mathrm{C}$ for $4 \mathrm{~h}$. After completion, purification by flash chromatography on silica gel $($ EtOAc/petroleum ether $=1 / 8-1 / 5)$ gave product 5a: $38.7 \mathrm{mg}$, as a white solid, yield $88 \% ;[\alpha]_{\mathrm{D}}^{20}:+52.0\left(c=0.32\right.$ in $\left.\mathrm{CHCl}_{3}\right) ; 93: 7$ er, determined by HPLC analysis [Daicel Chiralpak AD-H, $n$-hexane $/ i$-PrOH $=60 / 40,1.0 \mathrm{~mL} \mathrm{~min}^{-1}, \lambda$ $=254 \mathrm{~nm}]: \mathrm{t}($ major $)=8.08 \mathrm{~min}, \mathrm{t}($ minor $)=9.84 \mathrm{~min} ;{ }^{1} \mathrm{H} \mathrm{NMR}\left(600 \mathrm{MHz}, \mathrm{CDCl}_{3}\right): \delta(\mathrm{ppm}) 7.68(\mathrm{~d}$, $J=8.4 \mathrm{~Hz}, 1 \mathrm{H}), 7.55-7.53(\mathrm{~m}, 2 \mathrm{H}), 7.49(\mathrm{t}, J=8.6 \mathrm{~Hz}, 1 \mathrm{H}), 7.45-7.43(\mathrm{~m}, 3 \mathrm{H}), 7.27(\mathrm{t}, J=8.6 \mathrm{~Hz}$, $1 \mathrm{H}), 6.96(\mathrm{~d}, J=7.2 \mathrm{~Hz}, 1 \mathrm{H}), 5.93(\mathrm{dd}, J=12.6,7.2 \mathrm{~Hz}, 1 \mathrm{H}), 5.34(\mathrm{~d}, J=12.6 \mathrm{~Hz}, 1 \mathrm{H}), 3.55-3.52$ (m, 1H), 3.34-3.28 (m, 1H), $3.26(\mathrm{~s}, 3 \mathrm{H}), 2.95-2.90(\mathrm{~m}, 1 \mathrm{H}), 2.50(\mathrm{dd}, J=19.2,9.0 \mathrm{~Hz}, 1 \mathrm{H}), 2.33-$ $2.26(\mathrm{~m}, 1 \mathrm{H}), 1.82-1.77(\mathrm{~m}, 1 \mathrm{H}) ;{ }^{13} \mathrm{C}$ NMR $\left(150 \mathrm{MHz}, \mathrm{CDCl}_{3}\right): \delta(\mathrm{ppm}) 212.3,172.2,143.4,131.8$, 
131.3, 130.1, 129.5, 125.1, 124.5, 124.3, 111.6, 110.3, 109.4, 84.2, 53.1, 48.4, 48.3, 39.7, 38.4, 38.3, 27.0, 22.6; ESI-HRMS: calcd. for $\mathrm{C}_{25} \mathrm{H}_{20} \mathrm{~N}_{4} \mathrm{O}_{4}+\mathrm{Na}^{+} 463.1377$, found 463.1375 .

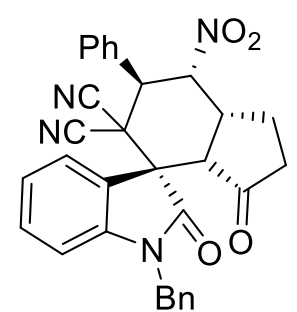

Synthesis of 5b: 2-Cyclopentenone 1a (16.4 mg, $0.2 \mathrm{mmol})$, 2-(1-benzyl-2oxoindolin-3-ylidene)malononitrile (28.5 mg, $0.1 \mathrm{mmol})$, PTC C2 (9.3 mg, 0.02 mmol), 2-mercaptobenzoic acid T1 (6.2 mg, $0.04 \mathrm{mmol})$ and $\mathrm{K}_{2} \mathrm{CO}_{3}(8.3 \mathrm{mg}, 0.06$ mmol) were stirred in distilled toluene $(1.0 \mathrm{~mL})$ at $\mathrm{rt}$ for $24 \mathrm{~h}$. Then $(E)-\beta-$ nitrostyrene $4 \mathbf{a}(14.9 \mathrm{mg}, 0.1 \mathrm{mmol})$ was added and the reaction was stirred at $60{ }^{\circ} \mathrm{C}$ for $4 \mathrm{~h}$. After completion, purification by flash chromatography on silica gel $($ EtOAc/petroleum ether $=1 / 8-1 / 5)$ gave product 5b: $41.2 \mathrm{mg}$, as a white solid, yield $80 \%$; $[\alpha]_{\mathrm{D}}{ }^{20}:+38.5\left(c=0.26\right.$ in $\left.\mathrm{CHCl}_{3}\right)$; $90.5: 9.5 \mathrm{er}$, determined by HPLC analysis [Daicel Chiralpak AD-H, $n$-hexane $/ i$-PrOH $=60 / 40,1.0 \mathrm{~mL} \mathrm{~min}^{-1}, \lambda$ $=254 \mathrm{~nm}]: \mathrm{t}($ major $)=6.28 \mathrm{~min}, \mathrm{t}($ minor $)=8.62 \mathrm{~min} ;{ }^{1} \mathrm{H} \mathrm{NMR}\left(600 \mathrm{MHz}, \mathrm{CDCl}_{3}\right): \delta(\mathrm{ppm}) 7.71(\mathrm{~d}$, $J=7.2 \mathrm{~Hz}, 1 \mathrm{H}), 7.57-7.55(\mathrm{~m}, 2 \mathrm{H}), 7.46-7.45(\mathrm{~m}, 3 \mathrm{H}), 7.42-7.38(\mathrm{~m}, 3 \mathrm{H}), 7.30-7.22(\mathrm{~m}, 4 \mathrm{H}), 6.79$ (d, $J=7.8 \mathrm{~Hz}, 1 \mathrm{H}), 5.95$ (dd, $J=13.2,7.2 \mathrm{~Hz}, 1 \mathrm{H}), 5.41$ (d, $J=12.6 \mathrm{~Hz}, 1 \mathrm{H}), 5.05$ (d, $J=16.2 \mathrm{~Hz}$, $1 \mathrm{H}), 4.88(\mathrm{~d}, J=16.2 \mathrm{~Hz}, 1 \mathrm{H}), 3.56-3.53(\mathrm{~m}, 1 \mathrm{H}), 3.33(\mathrm{~d}, J=9.0 \mathrm{~Hz}, 1 \mathrm{H}), 2.95-2.93(\mathrm{~m}, 1 \mathrm{H}), 2.45$ $(\mathrm{dd}, J=19.2,8.4 \mathrm{~Hz}, 1 \mathrm{H}), 2.32-2.30(\mathrm{~m}, 1 \mathrm{H}), 1.81-1.79(\mathrm{~m}, 1 \mathrm{H}) ;{ }^{13} \mathrm{C} \mathrm{NMR}\left(150 \mathrm{MHz}, \mathrm{CDCl}_{3}\right): \delta$ (ppm) 212.3, 172.4, 142.7, 134.0, 131.7, 131.2, 130.0, 129.5, 128.8, 127.9, 126.9, 125.0, 124.5, 124.3, 111.6, 110.5, 110.4, 84.1, 52.7, 48.8, 48.3, 44.8, 39.8, 38.5, 38.2, 22.5; ESI-HRMS: calcd. for $\mathrm{C}_{31} \mathrm{H}_{24} \mathrm{~N}_{4} \mathrm{O}_{4}+\mathrm{Na}^{+}$539.1690, found 539.1692.

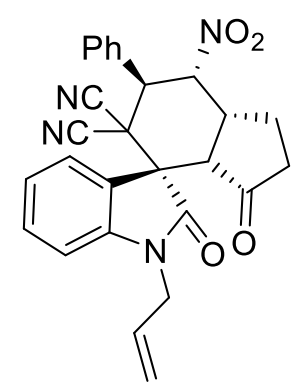

Synthesis of 5c: 2-Cyclopentenone $1 \mathrm{a}(16.4 \mathrm{mg}, 0.2 \mathrm{mmol})$, 2-(1-allyl-2oxoindolin-3-ylidene)malononitrile (20.9 mg, $0.1 \mathrm{mmol})$, PTC C2 (9.3 mg, 0.02 mmol), 2-mercaptobenzoic acid T1 (6.2 mg, $0.04 \mathrm{mmol})$ and $\mathrm{K}_{2} \mathrm{CO}_{3}(8.3 \mathrm{mg}, 0.06$ $\mathrm{mmol})$ were stirred in distilled toluene $(1.0 \mathrm{~mL})$ at $\mathrm{rt}$ for $24 \mathrm{~h}$. Then $(E)-\beta-$ nitrostyrene $4 \mathbf{a}(14.9 \mathrm{mg}, 0.1 \mathrm{mmol})$ was added and the reaction was stirred at 60 ${ }^{\circ} \mathrm{C}$ for $4 \mathrm{~h}$. After completion, purification by flash chromatography on silica gel (EtOAc/petroleum ether $=1 / 8-1 / 5)$ gave product $5 \mathbf{c}: 40.1 \mathrm{mg}$, as a white solid, yield $86 \% ;[\alpha]_{\mathrm{D}}^{20}:+62.3(c=0.41$ in $\mathrm{CHCl}_{3}$ ); 90.5:9.5 er, determined by HPLC analysis [Daicel Chiralpak AD-H, $n$-hexane/i-PrOH = 60/40, $\left.1.0 \mathrm{~mL} \min ^{-1}, \lambda=254 \mathrm{~nm}\right]: \mathrm{t}$ (major) $=5.98 \mathrm{~min}, \mathrm{t}$ (minor) $=7.72 \mathrm{~min} ;{ }^{1} \mathrm{H} \mathrm{NMR}(600 \mathrm{MHz}$, $\left.\mathrm{CDCl}_{3}\right): \delta(\mathrm{ppm}) 7.69(\mathrm{~d}, J=7.8 \mathrm{~Hz}, 1 \mathrm{H}), 7.56-7.53(\mathrm{~m}, 2 \mathrm{H}), 7.47-7.42(\mathrm{~m}, 4 \mathrm{H}), 7.26-7.25(\mathrm{~m}, 1 \mathrm{H})$, 
7.17-7.15 (m, 1H), $6.94(\mathrm{~d}, J=7.8 \mathrm{~Hz}, 1 \mathrm{H}), 5.94(\mathrm{dd}, J=12.0,7.2 \mathrm{~Hz}, 1 \mathrm{H}), 5.81-5.79(\mathrm{~m}, 1 \mathrm{H})$, 5.37-5.26 (m, 3H), 4.38-4.36 (m, 2H), 3.55-3.53 (m, 1H), 3.32-3.30 (m, 1H), 2.93-2.91 (m, 1H), $2.48(\mathrm{dd}, J=19.2,9.0 \mathrm{~Hz}, 1 \mathrm{H}), 2.33-2.31(\mathrm{~m}, 1 \mathrm{H}), 1.82-1.80(\mathrm{~m}, 1 \mathrm{H}) ;{ }^{13} \mathrm{C} \mathrm{NMR}\left(150 \mathrm{MHz}, \mathrm{CDCl}_{3}\right)$ : $\delta$ (ppm) 212.1, 172.0, 142.7, 131.8, 131.2, 130.1, 129.9, 129.5, 125.0, 124.5, 124.3, 118.6, 111.6, 110.4, 84.2, 52.7, 48.7, 48.3, 43.2, 39.8, 38.5, 38.2, 22.6; ESI-HRMS: calcd. for $\mathrm{C}_{27} \mathrm{H}_{22} \mathrm{~N}_{4} \mathrm{O}_{4}+\mathrm{Na}^{+}$ 489.1533, found 489.1532 .

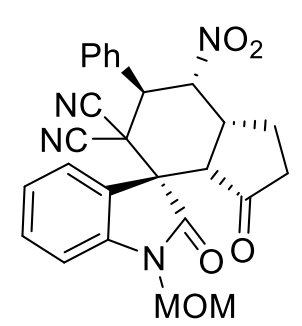

Synthesis of 5d: 2-Cyclopentenone 1a (16.4 $\mathrm{mg}, 0.2 \mathrm{mmol})$, 2-(1(methoxymethyl)-2-oxoindolin-3-ylidene)malononitrile (23.9 $\mathrm{mg}, 0.1 \mathrm{mmol}$ ), PTC C2 (9.3 mg, $0.02 \mathrm{mmol})$, 2-mercaptobenzoic acid T1 (6.2 mg, $0.04 \mathrm{mmol})$ and $\mathrm{K}_{2} \mathrm{CO}_{3}(8.3 \mathrm{mg}, 0.06 \mathrm{mmol})$ were stirred in distilled toluene $(1.0 \mathrm{~mL})$ at $\mathrm{rt}$ for $24 \mathrm{~h}$. Then $(E)-\beta$-nitrostyrene $\mathbf{4 a}(14.9 \mathrm{mg}, 0.1 \mathrm{mmol})$ was added and the reaction was stirred at $60{ }^{\circ} \mathrm{C}$ for $4 \mathrm{~h}$. After completion, purification by flash chromatography on silica gel $($ EtOAc/petroleum ether $=1 / 8-1 / 5)$ gave product 5d: $38.5 \mathrm{mg}$, as a white solid, yield $82 \% ;[\alpha]_{\mathrm{D}}{ }^{20}$ : +45.6 $\left(c=0.35\right.$ in $\left.\mathrm{CHCl}_{3}\right) ; 89.5: 10.5$ er, determined by HPLC analysis [Daicel Chiralpak AD-H, $n$ hexane $\left./ i-\mathrm{PrOH}=60 / 40,1.0 \mathrm{~mL} \min ^{-1}, \lambda=254 \mathrm{~nm}\right]: \mathrm{t}($ major $)=6.16 \mathrm{~min}, \mathrm{t}($ minor $)=7.44 \mathrm{~min} ;{ }^{1} \mathrm{H}$ NMR (600 MHz, $\left.\mathrm{CDCl}_{3}\right): \delta(\mathrm{ppm}) 7.71(\mathrm{~d}, J=8.8 \mathrm{~Hz}, 1 \mathrm{H}), 7.55-7.53(\mathrm{~m}, 2 \mathrm{H}), 7.49$ (t, $J=8.8 \mathrm{~Hz}$, 1H), 7.45-7.43 (m, 3H), $7.29(\mathrm{t}, J=8.8 \mathrm{~Hz}, 1 \mathrm{H}), 7.18(\mathrm{~d}, J=8.8 \mathrm{~Hz}, 1 \mathrm{H}), 5.95(\mathrm{dd}, J=12.6,7.2 \mathrm{~Hz}$, $1 \mathrm{H}), 5.29(\mathrm{~d}, J=12.6 \mathrm{~Hz}, 1 \mathrm{H}), 5.16(\mathrm{dd}, J=20.4,11.4 \mathrm{~Hz}, 2 \mathrm{H}), 3.56-3.54(\mathrm{~m}, 1 \mathrm{H}), 3.36(\mathrm{~s}, 3 \mathrm{H})$, 3.34-3.32 (m, 1H), 2.90-2.88 (m, 1H), $2.47(\mathrm{dd}, J=19.2,9.0 \mathrm{~Hz}, 1 \mathrm{H}), 2.35-2.33(\mathrm{~m}, 1 \mathrm{H}), 1.82-1.80$ $(\mathrm{m}, 1 \mathrm{H}) ;{ }^{13} \mathrm{C} \mathrm{NMR}\left(150 \mathrm{MHz}, \mathrm{CDCl}_{3}\right): \delta(\mathrm{ppm}) 212.3,173.2,142.0,131.7,131.5,130.1,129.5,124.7$, 124.5, 111.5, 110.9, 110.5, 84.2, 72.5, 56.8, 53.6, 48.8, 48.4, 39.9, 38.4, 38.4, 22.6; ESI-HRMS: calcd. for $\mathrm{C}_{26} \mathrm{H}_{22} \mathrm{~N}_{4} \mathrm{O}_{5}+\mathrm{Na}^{+}$493.1482, found 493.1485.

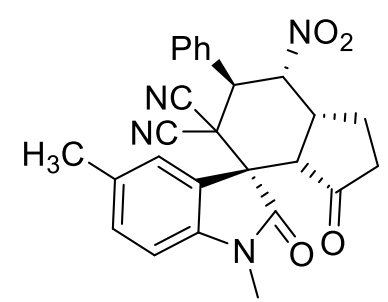

Synthesis of 5e: 2-Cyclopentenone $1 \mathrm{a}(16.4 \mathrm{mg}, 0.2 \mathrm{mmol})$, 2-(1,5dimethyl-2-oxoindolin-3-ylidene)malononitrile (22.3 mg, $0.1 \mathrm{mmol})$, PTC C2 (9.3 mg, $0.02 \mathrm{mmol}), 2$-mercaptobenzoic acid T1 (6.2 mg, $0.04 \mathrm{mmol})$ and $\mathrm{K}_{2} \mathrm{CO}_{3}(8.3 \mathrm{mg}, 0.06 \mathrm{mmol})$ were stirred in distilled toluene $(1.0 \mathrm{~mL})$ at rt for $24 \mathrm{~h}$. Then $(E)-\beta$-nitrostyrene $4 \mathbf{a}(14.9 \mathrm{mg}, 0.1 \mathrm{mmol})$ was added and the reaction was stirred at $60{ }^{\circ} \mathrm{C}$ for $4 \mathrm{~h}$. After completion, purification by flash chromatography on silica gel 
$($ EtOAc/petroleum ether $=1 / 8-1 / 5)$ gave product 5e: $36.3 \mathrm{mg}$, as a white solid, yield $80 \% ;[\alpha]_{\mathrm{D}}{ }^{20}$ : +73.4 ( $c=0.52$ in $\left.\mathrm{CHCl}_{3}\right)$; $91: 9$ er, determined by HPLC analysis [Daicel Chiralpak AD-H, $n$ hexane $\left./ i-\mathrm{PrOH}=60 / 40,1.0 \mathrm{~mL} \min ^{-1}, \lambda=254 \mathrm{~nm}\right]: \mathrm{t}($ major $)=6.44 \mathrm{~min}, \mathrm{t}($ minor $)=8.13 \mathrm{~min} ;{ }^{1} \mathrm{H}$ NMR (600 MHz, CDCl $): \delta(\mathrm{ppm}) 7.56-7.53(\mathrm{~m}, 2 \mathrm{H}), 7.47(\mathrm{~s}, 1 \mathrm{H}), 7.45-7.42(\mathrm{~m}, 3 \mathrm{H}), 7.28(\mathrm{~d}, J=$ $7.2 \mathrm{~Hz}, 1 \mathrm{H}), 6.84(\mathrm{~d}, J=7.2 \mathrm{~Hz}, 1 \mathrm{H}), 5.93(\mathrm{dd}, J=12.6,7.2 \mathrm{~Hz}, 1 \mathrm{H}), 5.36(\mathrm{~d}, J=12.0 \mathrm{~Hz}, 1 \mathrm{H}), 3.53-$ $3.51(\mathrm{~m}, 1 \mathrm{H}), 3.29(\mathrm{~d}, J=8.4 \mathrm{~Hz}, 1 \mathrm{H}), 3.23(\mathrm{~s}, 3 \mathrm{H}), 2.94-2.92(\mathrm{~m}, 1 \mathrm{H}), 2.50(\mathrm{dd}, J=19.2,9.0 \mathrm{~Hz}$, 1H), 2.41 (s, 3H), 2.31-2.29 (m, 1H), 1.81-1.79 (m, 1H); ${ }^{13} \mathrm{C}$ NMR (150 MHz, $\left.\mathrm{CDCl}_{3}\right): \delta(\mathrm{ppm})$ 212.3, 172.0, 141.0, 134.0, 131.9, 131.7, 130.6, 129.5, 125.2, 125.0, 111.6, 110.4, 109.1, 84.3, 53.1, 48.5, 48.4, 39.7, 38.5, 38.3, 27.0, 22.6, 21.4; ESI-HRMS: calcd. for $\mathrm{C}_{26} \mathrm{H}_{22} \mathrm{~N}_{4} \mathrm{O}_{4}+\mathrm{Na}^{+} 477.1533$, found 477.1532 .

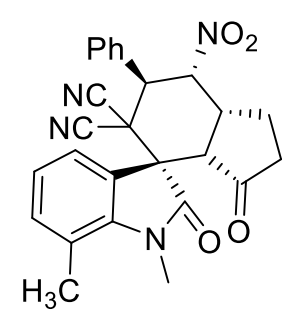

Synthesis of 5f: 2-Cyclopentenone 1a (16.4 mg, $0.2 \mathrm{mmol}), 2$-(1,7-dimethyl-2oxoindolin-3-ylidene)malononitrile (22.3 mg, $0.1 \mathrm{mmol})$, PTC C2 (9.3 mg, 0.02 mmol), 2-mercaptobenzoic acid T1 (6.2 mg, $0.04 \mathrm{mmol})$ and $\mathrm{K}_{2} \mathrm{CO}_{3}(8.3 \mathrm{mg}, 0.06$ mmol) were stirred in distilled toluene $(1.0 \mathrm{~mL})$ at $\mathrm{rt}$ for $24 \mathrm{~h}$. Then $(E)-\beta-$ nitrostyrene $4 \mathbf{a}(14.9 \mathrm{mg}, 0.1 \mathrm{mmol})$ was added and the reaction was stirred at 60 ${ }^{\circ} \mathrm{C}$ for $4 \mathrm{~h}$. After completion, purification by flash chromatography on silica gel (EtOAc/petroleum ether $=1 / 8-1 / 5)$ gave product $\mathbf{5 f}: 38.6 \mathrm{mg}$, as a white solid, yield $85 \% ;[\alpha]_{\mathrm{D}}{ }^{20}:+61.7(c=0.45$ in $\mathrm{CHCl}_{3}$ ); 92:8 er, determined by HPLC analysis [Daicel Chiralpak AD-H, $n$-hexane/ $i$-PrOH $=60 / 40$, $\left.1.0 \mathrm{~mL} \min ^{-1}, \lambda=254 \mathrm{~nm}\right]: \mathrm{t}$ (major) $=8.04 \mathrm{~min}, \mathrm{t}($ minor $)=9.47 \mathrm{~min} ;{ }^{1} \mathrm{H} \mathrm{NMR}\left(600 \mathrm{MHz}, \mathrm{CDCl}_{3}\right)$ : $\delta$ (ppm) 7.54-7.50 (m, 3H), 7.44-7.40 (m, 3H), 7.20 (d, J = 7.8 Hz, 1H), $7.13(\mathrm{t}, J=7.2 \mathrm{~Hz}, 1 \mathrm{H})$, $5.93(\mathrm{dd}, J=12.6,7.2 \mathrm{~Hz}, 1 \mathrm{H}), 5.36(\mathrm{~d}, J=12.0 \mathrm{~Hz}, 1 \mathrm{H}), 3.54-3.49(\mathrm{~m}, 4 \mathrm{H}), 3.29-3.26(\mathrm{~m}, 1 \mathrm{H})$, 2.95-2.93 (m, 1H), 2.59 (s, 3H), $2.50(\mathrm{dd}, J=19.2,9.0 \mathrm{~Hz}, 1 \mathrm{H}), 2.31-2.28(\mathrm{~m}, 1 \mathrm{H}), 1.81-1.78(\mathrm{~m}$, $1 \mathrm{H}) ;{ }^{13} \mathrm{C} \mathrm{NMR}\left(150 \mathrm{MHz}, \mathrm{CDCl}_{3}\right): \delta$ (ppm) 212.4, 172.9, 141.2, 135.2, 131.8, 130.0, 129.5, 125.6, 124.0, 122.3, 121.0, 111.6, 110.5, 84.2, 52.6, 48.7, 48.5, 39.8, 38.5, 38.3, 30.5, 22.6, 19.1; ESI-HRMS: calcd. for $\mathrm{C}_{26} \mathrm{H}_{22} \mathrm{~N}_{4} \mathrm{O}_{4}+\mathrm{Na}^{+} 477.1533$, found 477.1534. 


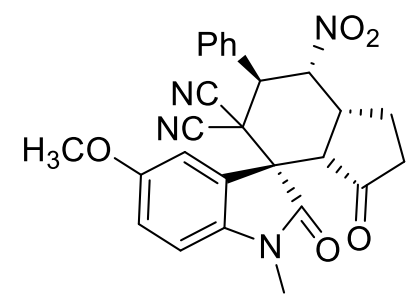

Synthesis of 5g: 2-Cyclopentenone 1a (16.4 mg, $0.2 \mathrm{mmol}), 2-(1-\mathrm{methyl}-$ 2-oxoindolin-3-ylidene)malononitrile (23.9 mg, $0.1 \mathrm{mmol})$, PTC C2 (9.3 $\mathrm{mg}, 0.02 \mathrm{mmol}), 2$-mercaptobenzoic acid T1 (6.2 $\mathrm{mg}, 0.04 \mathrm{mmol})$ and $\mathrm{K}_{2} \mathrm{CO}_{3}(8.3 \mathrm{mg}, 0.06 \mathrm{mmol})$ were stirred in distilled toluene $(1.0 \mathrm{~mL})$ at $\mathrm{rt}$ for $24 \mathrm{~h}$. Then $(E)-\beta$-nitrostyrene $\mathbf{4 a}(14.9 \mathrm{mg}, 0.1 \mathrm{mmol})$ was added and the reaction was stirred at $60{ }^{\circ} \mathrm{C}$ for $4 \mathrm{~h}$. After completion, purification by flash chromatography on silica gel (EtOAc/petroleum ether $=1 / 8-1 / 5)$ gave product $\mathbf{5 g}: 38.5 \mathrm{mg}$, as a white solid, yield $82 \% ;[\alpha]_{\mathrm{D}}{ }^{20}:+87.2(c=0.48$ in $\mathrm{CHCl}_{3}$ ); 96:4 er, determined by HPLC analysis [Daicel Chiralpak AD-H, $n$-hexane/i-PrOH = 60/40, $\left.1.0 \mathrm{~mL} \mathrm{~min}^{-1}, \lambda=254 \mathrm{~nm}\right]: \mathrm{t}($ major $)=11.23 \mathrm{~min}, \mathrm{t}($ minor $)=12.74 \mathrm{~min} ;{ }^{1} \mathrm{H} \mathrm{NMR}\left(600 \mathrm{MHz}, \mathrm{CDCl}_{3}\right)$ : $\delta(\mathrm{ppm}) 7.55-7.53(\mathrm{~m}, 2 \mathrm{H}), 7.47-7.43(\mathrm{~m}, 3 \mathrm{H}), 7.28(\mathrm{~s}, 1 \mathrm{H}), 7.00(\mathrm{t}, J=6.6 \mathrm{~Hz}, 1 \mathrm{H}), 6.86(\mathrm{~d}, J=6.6$ $\mathrm{Hz}, 1 \mathrm{H}), 5.93(\mathrm{dd}, J=12.6,7.2 \mathrm{~Hz}, 1 \mathrm{H}), 5.37(\mathrm{~d}, J=12.6 \mathrm{~Hz}, 1 \mathrm{H}), 3.84(\mathrm{~s}, 3 \mathrm{H}), 3.57-3.55(\mathrm{~m}, 1 \mathrm{H})$, 3.29-3.27 (m, 1H), $3.24(\mathrm{~s}, 3 \mathrm{H}), 2.95-2.93(\mathrm{~m}, 1 \mathrm{H}), 2.52(\mathrm{dd}, J=19.2,9.0 \mathrm{~Hz}, 1 \mathrm{H}), 2.32-2.30(\mathrm{~m}$, 1H), $1.83-1.80(\mathrm{~m}, 1 \mathrm{H}) ;{ }^{13} \mathrm{C} \mathrm{NMR}\left(150 \mathrm{MHz}, \mathrm{CDCl}_{3}\right): \delta$ (ppm) 212.2, 171.7, 156.9, 136.6, 131.8, 130.0, 129.5, 126.2, 115.5, 111.9, 111.5, 110.3, 109.9, 84.2, 55.8, 53.3, 48.5, 48.4, 39.7, 38.4, 38.3, 27.1, 22.6; ESI-HRMS: calcd. for $\mathrm{C}_{26} \mathrm{H}_{22} \mathrm{~N}_{4} \mathrm{O}_{5}+\mathrm{Na}^{+}$493.1482, found 493.1485 .

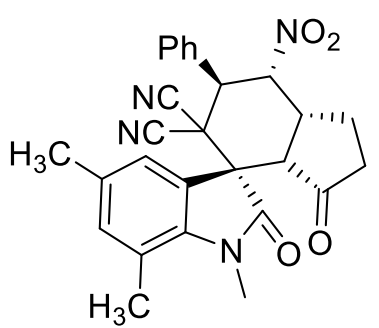

Synthesis of 5h: 2-Cyclopentenone 1a (16.4 mg, $0.2 \mathrm{mmol})$, 2-(1,5,7trimethyl-2-oxoindolin-3-ylidene)malononitrile (23.7 mg, $0.1 \mathrm{mmol})$, PTC C2 (9.3 mg, $0.02 \mathrm{mmol}), 2$-mercaptobenzoic acid T1 (6.2 mg, $0.04 \mathrm{mmol})$ and $\mathrm{K}_{2} \mathrm{CO}_{3}(8.3 \mathrm{mg}, 0.06 \mathrm{mmol})$ were stirred in distilled toluene $(1.0 \mathrm{~mL})$ at $\mathrm{rt}$ for $24 \mathrm{~h}$. Then $(E)-\beta$-nitrostyrene $4 \mathbf{a}(14.9 \mathrm{mg}, 0.1 \mathrm{mmol})$ was added and the reaction was stirred at $60{ }^{\circ} \mathrm{C}$ for $4 \mathrm{~h}$. After completion, purification by flash chromatography on silica gel $($ EtOAc/petroleum ether $=1 / 8-1 / 5)$ gave product $\mathbf{5 h}: 38.8 \mathrm{mg}$, as a white solid, yield 83\%; $[\alpha]_{\mathrm{D}}^{20}:+72.8\left(c=0.36\right.$ in $\left.\mathrm{CHCl}_{3}\right) ; 95.5: 4.5 \mathrm{er}$, determined by HPLC analysis [Daicel Chiralpak AD$\mathrm{H}, n$-hexane $/ i$-PrOH $\left.=60 / 40,1.0 \mathrm{~mL} \mathrm{~min}^{-1}, \lambda=254 \mathrm{~nm}\right]: \mathrm{t}$ (major) $=6.04 \mathrm{~min}, \mathrm{t}($ minor $)=8.03 \mathrm{~min}$; ${ }^{1} \mathrm{H}$ NMR (400 MHz, $\mathrm{CDCl}_{3}$ ): $\delta(\mathrm{ppm})$ 7.56-7.53 (m, 2H), 7.45-7.42 (m, 3H), 7.30 (s, 1H), 7.00 (s, 1H), $5.92(\mathrm{dd}, J=12.4,7.6 \mathrm{~Hz}, 1 \mathrm{H}), 5.37$ (d, $J=12.4 \mathrm{~Hz}, 1 \mathrm{H}), 3.55-3.48$ (m, 4H), 3.27-3.24 (m, 1H), 2.94-2.92 (m, 1H), 2.59 (s, 3H), 2.50-2.48 (m, 1H), 2.35 (s, 3H), 2.33-2.23 (m, 1H), 1.80-1.77 $(\mathrm{m}, 1 \mathrm{H}) ;{ }^{13} \mathrm{C} \mathrm{NMR}\left(150 \mathrm{MHz}, \mathrm{CDCl}_{3}\right): \delta(\mathrm{ppm}) 212.4,172.8,138.8,135.7,133.6,131.9,130.0,129.4$, $125.6,122.9,120.6,111.5,110.5,84.3,52.6$, 48.8, 48.5, 39.8, 38.4, 38.3, 30.3, 22.6, 21.1, 18.9; ESI- 
HRMS: calcd. for $\mathrm{C}_{27} \mathrm{H}_{24} \mathrm{~N}_{4} \mathrm{O}_{4}+\mathrm{Na}^{+} 491.1690$, found 491.1692 .

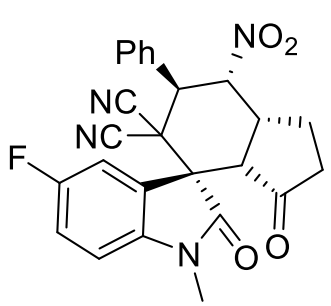

Synthesis of 5i: 2-Cyclopentenone 1a $(16.4 \mathrm{mg}, 0.2 \mathrm{mmol}), 2-(5-f l u o r o-1-$ methyl-2-oxoindolin-3-ylidene)malononitrile (22.7 mg, $0.1 \mathrm{mmol})$, PTC C2 (9.3 mg, $0.02 \mathrm{mmol})$, 2-mercaptobenzoic acid T1 (6.2 $\mathrm{mg}, 0.04 \mathrm{mmol})$ and $\mathrm{K}_{2} \mathrm{CO}_{3}(8.3 \mathrm{mg}, 0.06 \mathrm{mmol})$ were stirred in distilled toluene $(1.0 \mathrm{~mL})$ at $\mathrm{rt}$ for $24 \mathrm{~h}$. Then $(E)$ - $\beta$-nitrostyrene $4 \mathbf{a}(14.9 \mathrm{mg}, 0.1 \mathrm{mmol})$ was added and the reaction was stirred at 60 ${ }^{\circ} \mathrm{C}$ for $4 \mathrm{~h}$. After completion, purification by flash chromatography on silica gel (EtOAc/petroleum ether $=1 / 8-1 / 5)$ gave product $\mathbf{5 i}: 39.4 \mathrm{mg}$, as a white solid, yield $86 \% ;[\alpha]_{\mathrm{D}}{ }^{20}:+31.5(c=0.27$ in $\mathrm{CHCl}_{3}$ ); 83:17 er, determined by HPLC analysis [Daicel Chiralpak AD-H, $n$-hexane/i-PrOH $=60 / 40$, $\left.1.0 \mathrm{~mL} \min ^{-1}, \lambda=254 \mathrm{~nm}\right]: \mathrm{t}$ (major) $=7.81 \mathrm{~min}, \mathrm{t}($ minor $)=8.34 \mathrm{~min} ;{ }^{1} \mathrm{H} \mathrm{NMR}\left(600 \mathrm{MHz}, \mathrm{CDCl}_{3}\right)$ : $\delta(\mathrm{ppm}) 7.55-7.51(\mathrm{~m}, 2 \mathrm{H}), 7.46-7.43(\mathrm{~m}, 4 \mathrm{H}), 7.20(\mathrm{t}, J=8.6 \mathrm{~Hz}, 1 \mathrm{H}), 6.90(\mathrm{~d}, J=7.2 \mathrm{~Hz}, 1 \mathrm{H})$, $5.92(\mathrm{dd}, J=12.6,7.2 \mathrm{~Hz}, 1 \mathrm{H}), 5.35(\mathrm{~d}, J=12.6 \mathrm{~Hz}, 1 \mathrm{H}), 3.55-3.53(\mathrm{~m}, 1 \mathrm{H}), 3.25-3.23(\mathrm{~m}, 4 \mathrm{H})$, 2.94-2.92 (m, 1H), $2.50(\mathrm{dd}, J=19.2,9.0 \mathrm{~Hz}, 1 \mathrm{H}), 2.33-2.31(\mathrm{~m}, 1 \mathrm{H}), 1.82-1.80(\mathrm{~m}, 1 \mathrm{H}) ;{ }^{13} \mathrm{C} \mathrm{NMR}$ $\left(150 \mathrm{MHz}, \mathrm{CDCl}_{3}\right): \delta(\mathrm{ppm}) 212.1,171.9,159.8\left(\mathrm{~d},{ }^{1} J_{\mathrm{FC}}=240.7 \mathrm{~Hz}\right), 139.5,131.7\left(\mathrm{~d},{ }^{3} J_{\mathrm{FC}}=7.8 \mathrm{~Hz}\right)$, $130.2,129.6,129.5,126.7,126.6,118.0\left(\mathrm{~d},{ }^{2} J_{\mathrm{FC}}=23.4 \mathrm{~Hz}\right), 113.1\left(\mathrm{~d},{ }^{3} J_{\mathrm{FC}}=7.8 \mathrm{~Hz}\right), 111.5,110.3$, 110.2, 84.1, 53.3, 48.5, 48.2, 39.7, 38.4, 38.3, 27.2, 22.7; ESI-HRMS: calcd. for $\mathrm{C}_{25} \mathrm{H}_{19} \mathrm{FN}_{4} \mathrm{O}_{4}+\mathrm{Na}^{+}$ 481.1283 , found 481.1285 .

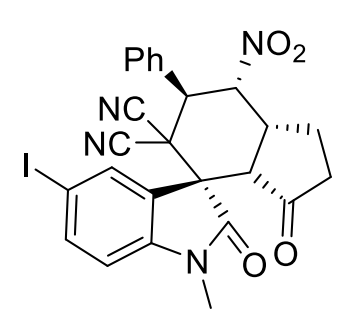

Synthesis of 5j: 2-Cyclopentenone 1a (16.4 mg, $0.2 \mathrm{mmol})$, 2-(5-iodo-1methyl-2-oxoindolin-3-ylidene)malononitrile (33.5 mg, $0.1 \mathrm{mmol})$, PTC C2 (9.3 mg, $0.02 \mathrm{mmol})$, 2-mercaptobenzoic acid T1 (6.2 mg, $0.04 \mathrm{mmol})$ and $\mathrm{K}_{2} \mathrm{CO}_{3}(8.3 \mathrm{mg}, 0.06 \mathrm{mmol})$ were stirred in distilled toluene $(1.0 \mathrm{~mL})$ at $\mathrm{rt}$ for $24 \mathrm{~h}$. Then $(E)$ - $\beta$-nitrostyrene $4 \mathbf{a}(14.9 \mathrm{mg}, 0.1 \mathrm{mmol})$ was added and the reaction was stirred at 60 ${ }^{\circ} \mathrm{C}$ for $4 \mathrm{~h}$. After completion, purification by flash chromatography on silica gel (EtOAc/petroleum ether $=1 / 8-1 / 5)$ gave product $\mathbf{5 j}: 50.9 \mathrm{mg}$, as a white solid, yield $90 \%$; $[\alpha]_{\mathrm{D}}{ }^{20}:+39.3(c=0.34$ in $\mathrm{CHCl}_{3}$ ); 88:12 er, determined by HPLC analysis [Daicel Chiralpak AD-H, $n$-hexane/i-PrOH $=60 / 40$, $\left.1.0 \mathrm{~mL} \min ^{-1}, \lambda=254 \mathrm{~nm}\right]: \mathrm{t}$ (major) $=12.11 \mathrm{~min}, \mathrm{t}($ minor $)=9.03 \mathrm{~min} ;{ }^{1} \mathrm{H} \mathrm{NMR}\left(600 \mathrm{MHz}, \mathrm{CDCl}_{3}\right)$ : $\delta(\mathrm{ppm}) 7.94(\mathrm{~s}, 1 \mathrm{H}), 7.81(\mathrm{~d}, J=7.2 \mathrm{~Hz}, 1 \mathrm{H}), 7.55-7.51(\mathrm{~m}, 2 \mathrm{H}), 7.46-7.43(\mathrm{~m}, 3 \mathrm{H}), 6.73(\mathrm{~d}, J=$ $7.2 \mathrm{~Hz}, 1 \mathrm{H}), 5.90(\mathrm{dd}, J=12.6,7.2 \mathrm{~Hz}, 1 \mathrm{H}), 5.28(\mathrm{~d}, J=12.6 \mathrm{~Hz}, 1 \mathrm{H}), 3.55-3.53(\mathrm{~m}, 1 \mathrm{H}), 3.31-3.23$ 
(m, 4H), 2.89-2.87 (m, 1H), 2.50 (dd, $J=19.2,9.0 \mathrm{~Hz}, 1 \mathrm{H}), 2.33-2.31$ (m, 1H), 1.81-1.79 (m, 1H); ${ }^{13} \mathrm{C}$ NMR (150 MHz, $\mathrm{CDCl}_{3}$ ): $\delta$ (ppm) 212.1, 171.7, 140.2, 133.3, 131.6, 130.2, 129.6, 127.4, 111.3, 110.6, 84.1, 48.3, 39.8, 38.4, 38.3, 29.7, 27.0, 22.8; ESI-HRMS: calcd. for $\mathrm{C}_{25} \mathrm{H}_{19} \mathrm{IN}_{4} \mathrm{O}_{4}+\mathrm{Na}^{+}$ 589.0343 , found 589.0346 .

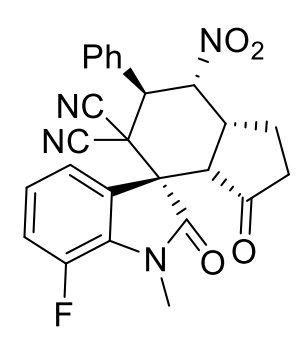

Synthesis of 5k: 2-Cyclopentenone 1a (16.4 mg, $0.2 \mathrm{mmol})$, 2-(7-fluoro-1methyl-2-oxoindolin-3-ylidene)malononitrile (22.7 mg, $0.1 \mathrm{mmol})$, PTC C2 (9.3 mg, $0.02 \mathrm{mmol}$ ), 2-mercaptobenzoic acid T1 (6.2 mg, $0.04 \mathrm{mmol})$ and $\mathrm{K}_{2} \mathrm{CO}_{3}(8.3$ $\mathrm{mg}, 0.06 \mathrm{mmol})$ were stirred in distilled toluene $(1.0 \mathrm{~mL})$ at $\mathrm{rt}$ for $24 \mathrm{~h}$. Then $(E)-$ $\beta$-nitrostyrene $4 \mathbf{a}(14.9 \mathrm{mg}, 0.1 \mathrm{mmol})$ was added and the reaction was stirred at $60{ }^{\circ} \mathrm{C}$ for $4 \mathrm{~h}$. After completion, purification by flash chromatography on silica gel (EtOAc/petroleum ether $=1 / 8-1 / 5)$ gave product 5k: $39.8 \mathrm{mg}$, as a white solid, yield $87 \%$; $[\alpha]_{\mathrm{D}}{ }^{20}:+43.6(c=0.31$ in $\mathrm{CHCl}_{3}$ ); 89:11 er, determined by HPLC analysis [Daicel Chiralpak AD-H, $n$-hexane/i-PrOH = 60/40, $\left.1.0 \mathrm{~mL} \min ^{-1}, \lambda=254 \mathrm{~nm}\right]: \mathrm{t}$ (major) $=6.36 \mathrm{~min}, \mathrm{t}($ minor $)=7.50 \mathrm{~min} ;{ }^{1} \mathrm{H} \mathrm{NMR}\left(600 \mathrm{MHz}, \mathrm{CDCl}_{3}\right)$ : $\delta(\mathrm{ppm}) 7.55-7.53(\mathrm{~m}, 2 \mathrm{H}), 7.49-7.43(\mathrm{~m}, 4 \mathrm{H}), 7.24-7.20(\mathrm{~m}, 2 \mathrm{H}), 5.92(\mathrm{dd}, J=12.0,7.2 \mathrm{~Hz}, 1 \mathrm{H})$, $5.32(\mathrm{~d}, J=12.0 \mathrm{~Hz}, 1 \mathrm{H}), 3.53-3.51(\mathrm{~m}, 1 \mathrm{H}), 3.46(\mathrm{~s}, 3 \mathrm{H}), 3.29-3.27(\mathrm{~m}, 1 \mathrm{H}), 2.93-2.91(\mathrm{~m}, 1 \mathrm{H})$, $2.52(\mathrm{dd}, J=19.2,9.0 \mathrm{~Hz}, 1 \mathrm{H}), 2.31-2.29(\mathrm{~m}, 1 \mathrm{H}), 1.81-1.79(\mathrm{~m}, 1 \mathrm{H}) ;{ }^{13} \mathrm{C} \mathrm{NMR}\left(100 \mathrm{MHz}, \mathrm{CDCl}_{3}\right)$ : $\delta(\mathrm{ppm}) 212.2,171.9,147.8\left(\mathrm{~d},{ }^{1} J_{\mathrm{FC}}=244.8 \mathrm{~Hz}\right), 131.6,130.4\left(\mathrm{~d},{ }^{2} J_{\mathrm{FC}}=9.1 \mathrm{~Hz}\right), 130.2,129.5,127.8$ $\left(\mathrm{d},{ }^{3} J_{\mathrm{FC}}=2.9 \mathrm{~Hz}\right), 124.9,124.8,120.3\left(\mathrm{~d},{ }^{3} J_{\mathrm{FC}}=3.4 \mathrm{~Hz}\right), 119.4\left(\mathrm{~d},{ }^{2} J_{\mathrm{FC}}=18.9 \mathrm{~Hz}\right), 111.4,110.2,84.1$, 53.3, 48.5, 48.3, 39.7, 38.3, 38.3, 29.6, 22.7; ESI-HRMS: calcd. for $\mathrm{C}_{25} \mathrm{H}_{19} \mathrm{FN}_{4} \mathrm{O}_{4}+\mathrm{Na}^{+} 481.1283$, found 481.1286 .

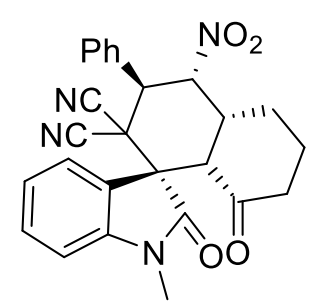

Synthesis of 5l: 2-Cyclohexenone $\mathbf{1 b}(19.2 \mathrm{mg}, 0.2 \mathrm{mmol}), 2-(1-\mathrm{methyl}-2-$ oxoindolin-3-ylidene)malononitrile $\mathbf{2 a}(20.9 \mathrm{mg}, 0.1 \mathrm{mmol})$, PTC C2 (9.3 mg, $0.02 \mathrm{mmol}), 2$-mercaptobenzoic acid $\mathrm{T1}(6.2 \mathrm{mg}, 0.04 \mathrm{mmol})$ and $\mathrm{K}_{2} \mathrm{CO}_{3}(8.3$ $\mathrm{mg}, 0.06 \mathrm{mmol})$ were stirred in distilled toluene $(1.0 \mathrm{~mL})$ at $\mathrm{rt}$ for $24 \mathrm{~h}$. Then $(E)-$ $\beta$-nitrostyrene $4 \mathbf{a}(14.9 \mathrm{mg}, 0.1 \mathrm{mmol})$ was added and the reaction was stirred at $60{ }^{\circ} \mathrm{C}$ for $4 \mathrm{~h}$. After completion, purification by flash chromatography on silica gel (EtOAc/petroleum ether $=1 / 8-1 / 5)$ gave product 5l: $19.0 \mathrm{mg}$, as a white solid, yield $42 \%$; $[\alpha]_{\mathrm{D}}{ }^{20}:+25.4\left(c=0.22\right.$ in $\left.\mathrm{CHCl}_{3}\right)$; 68:32 er, determined by HPLC analysis [Daicel Chiralpak AD-H, $n$-hexane $/ i$-PrOH $=60 / 40,1.0 \mathrm{~mL} \mathrm{~min}^{-1}, \lambda$ 
$=254 \mathrm{~nm}]: \mathrm{t}($ major $)=9.68 \mathrm{~min}, \mathrm{t}($ minor $)=16.5 \mathrm{~min} ;{ }^{1} \mathrm{H} \mathrm{NMR}\left(600 \mathrm{MHz}, \mathrm{CDCl}_{3}\right): \delta(\mathrm{ppm}) 7.74(\mathrm{~d}$, $J=7.8 \mathrm{~Hz}, 1 \mathrm{H}), 7.53-7.51(\mathrm{~m}, 2 \mathrm{H}), 7.47-7.42(\mathrm{~m}, 4 \mathrm{H}), 7.27(\mathrm{t}, J=8.6 \mathrm{~Hz}, 1 \mathrm{H}), 6.91(\mathrm{~d}, J=7.2 \mathrm{~Hz}$, $1 \mathrm{H}), 5.68(\mathrm{dd}, J=12.6,7.2 \mathrm{~Hz}, 1 \mathrm{H}), 5.45(\mathrm{~d}, J=12.0 \mathrm{~Hz}, 1 \mathrm{H}), 3.53-3.51(\mathrm{~m}, 1 \mathrm{H}), 3.32(\mathrm{~s}, 3 \mathrm{H}), 3.25-$ $3.23(\mathrm{~m}, 1 \mathrm{H}), 3.04-3.02(\mathrm{~m}, 1 \mathrm{H}), 2.29-2.18(\mathrm{~m}, 3 \mathrm{H}), 1.65-1.63(\mathrm{~m}, 1 \mathrm{H}), 1.35-1.32(\mathrm{~m}, 1 \mathrm{H}) ;{ }^{13} \mathrm{C}$ NMR (150 MHz, $\left.\mathrm{CDCl}_{3}\right): \delta$ (ppm) 203.5, 172.5, 142.4, 132.2, 131.3, 129.9, 129.4, 125.8, 124.2, 123.3, 111.5, 110.7, 109.3, 85.3, 53.3, 52.9, 49.0, 40.7, 40.4, 38.9, 27.1, 24.0, 20.7; ESI-HRMS: calcd. for $\mathrm{C}_{26} \mathrm{H}_{22} \mathrm{~N}_{4} \mathrm{O}_{4}+\mathrm{Na}^{+} 477.1533$, found 477.1532.

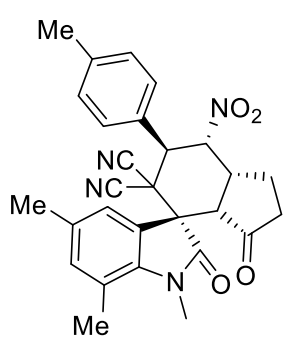

Synthesis of 5m: 2-Cyclopentenone 1a (16.4 mg, $0.2 \mathrm{mmol})$, 2-(1,5,7-trimethyl2-oxoindolin-3-ylidene)malononitrile (23.7 mg, $0.1 \mathrm{mmol})$, PTC C2 (9.3 mg, 0.02 mmol), 2-mercaptobenzoic acid T1 (6.2 mg, $0.04 \mathrm{mmol})$ and $\mathrm{K}_{2} \mathrm{CO}_{3}(8.3 \mathrm{mg}, 0.06$ $\mathrm{mmol})$ were stirred in distilled toluene $(1.0 \mathrm{~mL})$ at $\mathrm{rt}$ for $24 \mathrm{~h}$. Then $(E)-1$-methyl4-(2-nitrovinyl)benzene $(16.3 \mathrm{mg}, 0.1 \mathrm{mmol})$ was added and the reaction was stirred at $60{ }^{\circ} \mathrm{C}$ for $4 \mathrm{~h}$. After completion, purification by flash chromatography on silica gel $($ EtOAc/petroleum ether $=1 / 8-1 / 5)$ gave product 5m: $39.8 \mathrm{mg}$, as a white solid, yield $82 \% ;[\alpha]_{\mathrm{D}}{ }^{20}$ : $+83.7\left(c=0.35\right.$ in $\left.\mathrm{CHCl}_{3}\right)$; 93:7 er, determined by HPLC analysis [Daicel Chiralpak AD-H, $n$ hexane $\left./ i-\mathrm{PrOH}=60 / 40,1.0 \mathrm{~mL} \min ^{-1}, \lambda=254 \mathrm{~nm}\right]: \mathrm{t}($ major $)=6.57 \mathrm{~min}, \mathrm{t}($ minor $)=9.30 \mathrm{~min} ;{ }^{1} \mathrm{H}$ NMR (600 MHz, $\left.\mathrm{CDCl}_{3}\right): \delta(\mathrm{ppm}) 7.41(\mathrm{~d}, J=11.4 \mathrm{~Hz}, 2 \mathrm{H}), 7.30(\mathrm{~s}, 1 \mathrm{H}), 7.23(\mathrm{~d}, J=11.4 \mathrm{~Hz}, 1 \mathrm{H})$, $7.0(\mathrm{~s}, 1 \mathrm{H}), 5.90(\mathrm{dd}, J=12.0,7.2 \mathrm{~Hz}, 1 \mathrm{H}), 5.32(\mathrm{~d}, J=12.0 \mathrm{~Hz}, 1 \mathrm{H}), 3.53-3.45(\mathrm{~m}, 4 \mathrm{H}), 3.25-3.23$ (m, 1H), 2.95-2.93 (m, 1H), 2.54-2.46 (m, 4H), 2.35-2.25 (m, 7H), 1.77-1.75 (m, 1H); ${ }^{13} \mathrm{C}$ NMR (150 MHz, $\left.\mathrm{CDCl}_{3}\right): \delta$ (ppm) 212.5, 172.8, 140.0, 138.8, 135.7, 133.6, 130.1, 128.8, 125.7, 123.0, 120.6, 111.6, 110.6, 84.4, 52.6, 48.9, 48.5, 39.5, 38.5, 38.3, 30.4, 22.6, 21.2, 21.1, 18.9; ESI-HRMS: calcd. for $\mathrm{C}_{28} \mathrm{H}_{26} \mathrm{~N}_{4} \mathrm{O}_{4}+\mathrm{Na}^{+}$505.1846, found 505.1848.

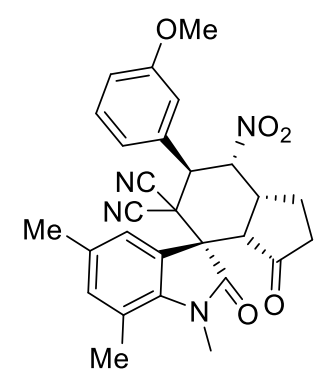

Synthesis of 5n: 2-Cyclopentenone 1a (16.4 mg, $0.2 \mathrm{mmol})$, 2-(1,5,7-trimethyl2-oxoindolin-3-ylidene)malononitrile (23.7 mg, $0.1 \mathrm{mmol})$, PTC C2 $(9.3 \mathrm{mg}$, $0.02 \mathrm{mmol}), 2$-mercaptobenzoic acid $\mathrm{T1}(6.2 \mathrm{mg}, 0.04 \mathrm{mmol})$ and $\mathrm{K}_{2} \mathrm{CO}_{3}(8.3$ $\mathrm{mg}, 0.06 \mathrm{mmol})$ were stirred in distilled toluene $(1.0 \mathrm{~mL})$ at $\mathrm{rt}$ for $24 \mathrm{~h}$. Then $(E)$ 1-methoxy-3-(2-nitrovinyl)benzene $(17.9 \mathrm{mg}, 0.1 \mathrm{mmol})$ was added and the reaction was stirred at $60{ }^{\circ} \mathrm{C}$ for $4 \mathrm{~h}$. After completion, purification by flash chromatography on silica 
gel $($ EtOAc/petroleum ether $=1 / 8-1 / 5)$ gave product $5 \mathrm{n}: 40.8 \mathrm{mg}$, as a white solid, yield $82 \%$; $[\alpha]_{\mathrm{D}}{ }^{20}$ : +76.2 ( $c=0.28$ in $\left.\mathrm{CHCl}_{3}\right)$; 94:6 er, determined by HPLC analysis [Daicel Chiralpak AD-H, $n$ hexane $\left./ i-\mathrm{PrOH}=60 / 40,1.0 \mathrm{~mL} \min ^{-1}, \lambda=254 \mathrm{~nm}\right]: \mathrm{t}($ major $)=6.16 \mathrm{~min}, \mathrm{t}($ minor $)=20.20 \mathrm{~min} ;{ }^{1} \mathrm{H}$ $\operatorname{NMR}\left(600 \mathrm{MHz}, \mathrm{CDCl}_{3}\right): \delta(\mathrm{ppm}) 7.37-7.30(\mathrm{~m}, 2 \mathrm{H}), 7.16-7.13(\mathrm{~m}, 1 \mathrm{H}), 7.05-7.03(\mathrm{~m}, 2 \mathrm{H}), 6.97-$ $6.95(\mathrm{~m}, 1 \mathrm{H}), 5.90(\mathrm{dd}, J=11.4,7.2 \mathrm{~Hz}, 1 \mathrm{H}), 5.35$ (d, $J=12.0 \mathrm{~Hz}, 1 \mathrm{H}), 3.85(\mathrm{~s}, 3 \mathrm{H}), 3.55-3.50(\mathrm{~m}$, 4H), 3.27-3.25 (m, 1H), 2.96-2.94 (m, 1H), $2.58(\mathrm{~s}, 3 \mathrm{H}), 2.55(\mathrm{dd}, J=18.6,8.4 \mathrm{~Hz}, 1 \mathrm{H}), 2.39$ (s, 3H), 2.32-2.30 (m, 1H), 1.81-1.79 (m, 1H); $\left.{ }^{13} \mathrm{C} \mathrm{NMR} \mathrm{(150} \mathrm{MHz,} \mathrm{CDCl}_{3}\right): \delta$ (ppm) 212.5, 172.8, $140.1,138.8,135.8,133.4,130.5,125.7,123.0,120.6,115.6,84.4,55.3,52.7,48.6,39.8,38.5,38.3$, 30.4, 22.6, 21.1, 18.9; ESI-HRMS: calcd. for $\mathrm{C}_{28} \mathrm{H}_{26} \mathrm{~N}_{4} \mathrm{O}_{5}+\mathrm{Na}^{+}$521.1795, found 521.1796.

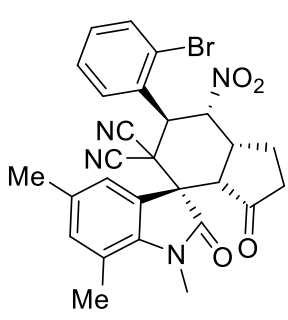

Synthesis of 5o: 2-Cyclopentenone 1a (16.4 mg, $0.2 \mathrm{mmol})$, 2-(1,5,7-trimethyl2-oxoindolin-3-ylidene)malononitrile (23.7 mg, $0.1 \mathrm{mmol})$, PTC C2 (9.3 mg, 0.02 mmol), 2-mercaptobenzoic acid $\mathbf{T 1}(6.2 \mathrm{mg}, 0.04 \mathrm{mmol})$ and $\mathrm{K}_{2} \mathrm{CO}_{3}(8.3 \mathrm{mg}, 0.06$ $\mathrm{mmol})$ were stirred in distilled toluene $(1.0 \mathrm{~mL})$ at $\mathrm{rt}$ for $24 \mathrm{~h}$. Then $(E)$-1-bromo2-(2-nitrovinyl)benzene $(22.7 \mathrm{mg}, 0.1 \mathrm{mmol})$ was added and the reaction was stirred at $60{ }^{\circ} \mathrm{C}$ for $4 \mathrm{~h}$. After completion, purification by flash chromatography on silica gel $($ EtOAc/petroleum ether $=1 / 8-1 / 5)$ gave product 5o: $40.9 \mathrm{mg}$, as a white solid, yield $75 \% ;[\alpha]_{\mathrm{D}}{ }^{20}$ : $+85.3\left(c=0.42\right.$ in $\left.\mathrm{CHCl}_{3}\right)$; 92.5:7.5 er, determined by HPLC analysis [Daicel Chiralpak AD-H, $n$ hexane $\left./ i-\mathrm{PrOH}=60 / 40,1.0 \mathrm{~mL} \min ^{-1}, \lambda=254 \mathrm{~nm}\right]: \mathrm{t}$ (major) $=6.15 \mathrm{~min}, \mathrm{t}($ minor $)=8.19 \mathrm{~min} ;{ }^{1} \mathrm{H}$ NMR (600 MHz, $\left.\mathrm{CDCl}_{3}\right): \delta(\mathrm{ppm}) 7.74(\mathrm{~d}, J=8.4 \mathrm{~Hz}, 1 \mathrm{H}), 7.70(\mathrm{~d}, J=8.4 \mathrm{~Hz}, 1 \mathrm{H}), 7.43(\mathrm{t}, J=8.4$ $\mathrm{Hz}, 1 \mathrm{H}), 7.32-7.29(\mathrm{~m}, 2 \mathrm{H}), 7.03(\mathrm{~s}, 1 \mathrm{H}), 6.28(\mathrm{~d}, J=7.2 \mathrm{~Hz}, 1 \mathrm{H}), 5.88(\mathrm{dd}, J=12.6,7.2 \mathrm{~Hz}, 1 \mathrm{H})$, 3.59-3.54 (m, 1H), 3.52 (s, 3H), 3.30-3.28 (m, 1H), 3.06-3.04 (m, 1H), 2.61-2.51 (m, 4H), 2.37-2.30 (m, 4H), 1.84-1.82 (m, 1H); $\left.{ }^{13} \mathrm{C} \mathrm{NMR} \mathrm{(150} \mathrm{MHz,} \mathrm{CDCl}_{3}\right): \delta(\mathrm{ppm}) 212.6,172.3,139.0,135.8,134.7$, 133.5, 131.8, 131.1, 128.3, 127.9, 126.8, 125.5, 122.9, 120.6, 111.6, 109.8, 84.9, 52.8, 48.4, 47.9, 38.6, 38.3, 37.6, 30.4, 22.7, 21.1, 18.9; ESI-HRMS: calcd. for $\mathrm{C}_{27} \mathrm{H}_{23} \mathrm{Br}^{79} \mathrm{~N}_{4} \mathrm{O}_{4}+\mathrm{Na}^{+}$569.0795, found 569.0798, calcd. for $\mathrm{C}_{27} \mathrm{H}_{23} \mathrm{Br}^{81} \mathrm{~N}_{4} \mathrm{O}_{4}+\mathrm{Na}^{+}$571.0774, found 571.0776. 


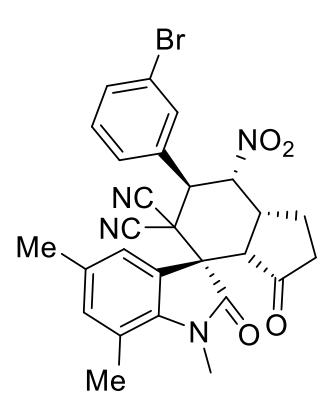

Synthesis of 5p: 2-Cyclopentenone 1a (16.4 mg, $0.2 \mathrm{mmol})$, 2-(1,5,7-trimethyl2-oxoindolin-3-ylidene)malononitrile (23.7 mg, $0.1 \mathrm{mmol})$, PTC C2 (9.3 mg, $0.02 \mathrm{mmol}), 2$-mercaptobenzoic acid $\mathbf{T 1}(6.2 \mathrm{mg}, 0.04 \mathrm{mmol})$ and $\mathrm{K}_{2} \mathrm{CO}_{3}(8.3$ $\mathrm{mg}, 0.06 \mathrm{mmol})$ were stirred in distilled toluene $(1.0 \mathrm{~mL})$ at $\mathrm{rt}$ for $24 \mathrm{~h}$. Then (E)-1-bromo-3-(2-nitrovinyl)benzene $(22.7 \mathrm{mg}, 0.1 \mathrm{mmol})$ was added and the reaction was stirred at $60{ }^{\circ} \mathrm{C}$ for $4 \mathrm{~h}$. After completion, purification by flash chromatography on silica gel (EtOAc/petroleum ether $=1 / 8-1 / 5)$ gave product $5 \mathbf{p}$ : $41.5 \mathrm{mg}$, as a white solid, yield $76 \% ;[\alpha]_{\mathrm{D}}^{20}:+70.0\left(c=0.34\right.$ in $\left.\mathrm{CHCl}_{3}\right)$; 94:6 er, determined by HPLC analysis [Daicel Chiralpak AD-H, $n$-hexane $/ i-\mathrm{PrOH}=60 / 40,1.0 \mathrm{~mL} \mathrm{~min}^{-1}, \lambda=254 \mathrm{~nm}$ ]: $\mathrm{t}$ (major) $=5.60 \mathrm{~min}$, $\mathrm{t}($ minor $)=8.60 \mathrm{~min} ;{ }^{1} \mathrm{H} \mathrm{NMR}\left(600 \mathrm{MHz}, \mathrm{CDCl}_{3}\right): \delta(\mathrm{ppm}) 7.69(\mathrm{~s}, 1 \mathrm{H}), 7.59-7.57(\mathrm{~m}, 1 \mathrm{H}), 7.52-$ $7.50(\mathrm{~m}, 1 \mathrm{H}), 7.35-7.33(\mathrm{~m}, 2 \mathrm{H}), 7.04(\mathrm{~s}, 1 \mathrm{H}), 5.86(\mathrm{dd}, J=12.6,7.2 \mathrm{~Hz}, 1 \mathrm{H}), 5.39(\mathrm{~d}, J=12.6 \mathrm{~Hz}$, $1 \mathrm{H}), 3.56-3.53(\mathrm{~m}, 4 \mathrm{H}), 3.27-3.25(\mathrm{~m}, 1 \mathrm{H}), 2.93-2.91(\mathrm{~m}, 1 \mathrm{H}), 2.58(\mathrm{~s}, 3 \mathrm{H}), 2.52(\mathrm{dd}, J=19.2,9.0$ $\mathrm{Hz}, 1 \mathrm{H}), 2.40-2.38(\mathrm{~s}, 3 \mathrm{H}), 2.37-2.27(\mathrm{~m}, 1 \mathrm{H}), 1.81-1.79(\mathrm{~m}, 1 \mathrm{H}) ;{ }^{13} \mathrm{C} \mathrm{NMR}\left(150 \mathrm{MHz}, \mathrm{CDCl}_{3}\right): \delta$ (ppm) 212.2, 172.7, 138.6, 135.9, 134.3, 133.7, 133.3, 130.9, 125.5, 123.5, 122.9, 120.7, 111.3, 110.3, $84.2, \quad 52.5,48.5,48.5,39.4,38.4,38.3,30.4,22.6,21.1,18.9$; ESI-HRMS: calcd. for $\mathrm{C}_{27} \mathrm{H}_{23} \mathrm{Br}^{79} \mathrm{~N}_{4} \mathrm{O}_{4}+\mathrm{Na}^{+}$569.0795, found 569.0796, calcd. for $\mathrm{C}_{27} \mathrm{H}_{23} \mathrm{Br}^{81} \mathrm{~N}_{4} \mathrm{O}_{4}+\mathrm{Na}^{+}$571.0774, found 571.0773.

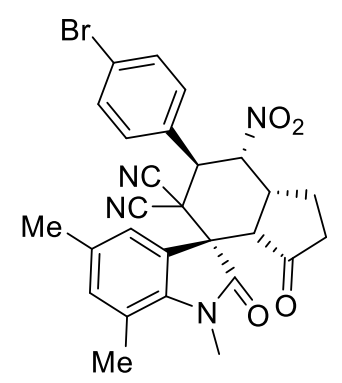

Synthesis of 5q: 2-Cyclopentenone 1a (16.4 mg, $0.2 \mathrm{mmol})$, 2-(1,5,7-trimethyl2-oxoindolin-3-ylidene)malononitrile (23.7 mg, $0.1 \mathrm{mmol})$, PTC C2 (9.3 mg, $0.02 \mathrm{mmol}), 2$-mercaptobenzoic acid $\mathbf{T 1}(6.2 \mathrm{mg}, 0.04 \mathrm{mmol})$ and $\mathrm{K}_{2} \mathrm{CO}_{3}(8.3$ $\mathrm{mg}, 0.06 \mathrm{mmol})$ were stirred in distilled toluene $(1.0 \mathrm{~mL})$ at $\mathrm{rt}$ for $24 \mathrm{~h}$. Then (E)-1-bromo-4-(2-nitrovinyl)benzene $(22.7 \mathrm{mg}, 0.1 \mathrm{mmol})$ was added and the reaction was stirred at $60{ }^{\circ} \mathrm{C}$ for $4 \mathrm{~h}$. After completion, purification by flash chromatography on silica gel (EtOAc/petroleum ether =1/8-1/5) gave product 5q: $42.6 \mathrm{mg}$, as a white solid, yield 78\%; $[\alpha]_{\mathrm{D}}{ }^{20}:+75.8\left(c=0.37\right.$ in $\left.\mathrm{CHCl}_{3}\right)$; 93.5:6.5 er, determined by HPLC analysis [Daicel Chiralpak AD-H, $n$-hexane $/ i$-PrOH $=60 / 40,1.0 \mathrm{~mL} \mathrm{~min}^{-1}, \lambda=254 \mathrm{~nm}$ ]: $\mathrm{t}$ (major) $=15.87$ min, $\mathrm{t}($ minor $)=17.88 \mathrm{~min} ;{ }^{1} \mathrm{H}$ NMR $\left(600 \mathrm{MHz}, \mathrm{CDCl}_{3}\right): \delta(\mathrm{ppm}) 7.58(\mathrm{~d}, J=8.4 \mathrm{~Hz}, 1 \mathrm{H}), 7.41(\mathrm{~d}$, $J=8.4 \mathrm{~Hz}, 1 \mathrm{H}), 7.29(\mathrm{~s}, 1 \mathrm{H}), 7.01(\mathrm{~s}, 1 \mathrm{H}), 5.85(\mathrm{dd}, J=12.0,7.2 \mathrm{~Hz}, 1 \mathrm{H}), 5.36(\mathrm{~d}, J=12.0 \mathrm{~Hz}, 1 \mathrm{H})$, 3.56-3.45 (m, 4H), 3.25-3.23 (m, 1H), 2.93-2.91 (m, 1H), 2.59-2.46 (m, 4H), 2.35-2.23 (m, 4H), 
1.80-1.78 (m, 1H); ${ }^{13} \mathrm{C}$ NMR (100 MHz, $\left.\mathrm{CDCl}_{3}\right): \delta(\mathrm{ppm}) 212.2,172.7,138.7,135.8,133.7,132.7$, $131.0,125.5,124.4,122.9,120.7,111.4,110.4,84.2,52.5,48.5,48.4,39.4,38.3,38.2,30.3,22.5$, 21.1, 18.9; ESI-HRMS: calcd. for $\mathrm{C}_{27} \mathrm{H}_{23} \mathrm{Br}^{79} \mathrm{~N}_{4} \mathrm{O}_{4}+\mathrm{Na}^{+}$569.0795, found 569.0797, calcd. for $\mathrm{C}_{27} \mathrm{H}_{23} \mathrm{Br}^{81} \mathrm{~N}_{4} \mathrm{O}_{4}+\mathrm{Na}^{+}$571.0774, found 571.0775.

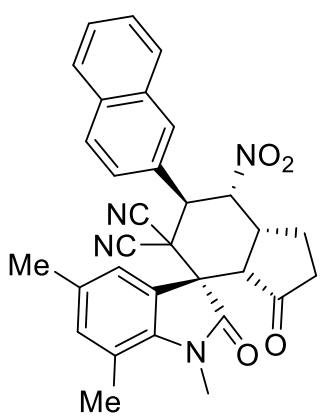

Synthesis of 5r: 2-Cyclopentenone 1a (16.4 mg, 0.2 mmol), 2-(1,5,7-trimethyl2-oxoindolin-3-ylidene)malononitrile (23.7 mg, $0.1 \mathrm{mmol})$, PTC C2 (9.3 mg, $0.02 \mathrm{mmol}), 2-$ mercaptobenzoic acid $\mathrm{T1}(6.2 \mathrm{mg}, 0.04 \mathrm{mmol})$ and $\mathrm{K}_{2} \mathrm{CO}_{3}(8.3$ $\mathrm{mg}, 0.06 \mathrm{mmol})$ were stirred in distilled toluene $(1.0 \mathrm{~mL})$ at $\mathrm{rt}$ for $24 \mathrm{~h}$. Then (E)-2-(2-nitrovinyl)naphthalene (19.9 $\mathrm{mg}, 0.1 \mathrm{mmol})$ was added and the reaction was stirred at $60{ }^{\circ} \mathrm{C}$ for $4 \mathrm{~h}$. After completion, purification by flash chromatography on silica gel $($ EtOAc/petroleum ether $=1 / 8-1 / 5)$ gave product $5 \mathbf{r}: 36.7 \mathrm{mg}$, as a white solid, yield 71\%; $[\alpha]_{\mathrm{D}}^{20}:+68.5\left(c=0.29\right.$ in $\left.\mathrm{CHCl}_{3}\right)$; 94:6 er, determined by HPLC analysis [Daicel Chiralpak AD-H, $n$-hexane $\left./ i-\mathrm{PrOH}=60 / 40,1.0 \mathrm{~mL} \mathrm{~min}^{-1}, \lambda=254 \mathrm{~nm}\right]: \mathrm{t}$ (major) $=8.00 \mathrm{~min}, \mathrm{t}$ (minor) $=17.7 \mathrm{~min} ;{ }^{1} \mathrm{H}$ NMR $\left(600 \mathrm{MHz}, \mathrm{CDCl}_{3}\right): \delta(\mathrm{ppm}) 8.06(\mathrm{~s}, 1 \mathrm{H}), 7.92-7.90(\mathrm{~m}, 2 \mathrm{H}), 7.87-7.85(\mathrm{~m}$, 1H), 7.64-7.62 (m, 1H), 7.56-7.54 (m, 2H), $7.35(\mathrm{~s}, 1 \mathrm{H}), 7.03(\mathrm{~s}, 1 \mathrm{H}), 6.08(\mathrm{dd}, J=12.0,7.2 \mathrm{~Hz}$, $1 \mathrm{H}), 5.58(\mathrm{~d}, J=12.0 \mathrm{~Hz}, 1 \mathrm{H}), 3.58-3.56(\mathrm{~m}, 1 \mathrm{H}), 3.53(\mathrm{~s}, 3 \mathrm{H}), 3.33-3.31(\mathrm{~m}, 1 \mathrm{H}), 3.03-3.01(\mathrm{~m}$, 1H), 2.56-2.51 (m, 4H), 2.38-2.30 (m, 4H), 1.83-1.81 (m, 1H); $\left.{ }^{13} \mathrm{C} \mathrm{NMR} \mathrm{(150} \mathrm{MHz,} \mathrm{CDCl}_{3}\right): \delta$ (ppm) 212.4, 172.9, 138.9, 135.8, 133.8, 133.7, 133.2, 129.5, 128.4, 127.7, 127.2, 126.8, 125.7, 123.0, 120.7, 111.7, 110.6, 84.6, 52.8, 48.8, 48.6, 39.9, 38.6, 38.4, 30.4, 22.7, 21.1, 18.9; ESI-HRMS: calcd. for $\mathrm{C}_{31} \mathrm{H}_{26} \mathrm{~N}_{4} \mathrm{O}_{4}+\mathrm{Na}^{+}$541.1846, found 541.1845.

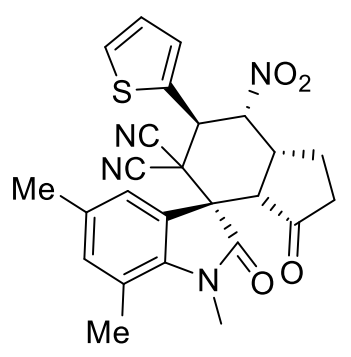

Synthesis of 5s: 2-Cyclopentenone 1a (16.4 mg, $0.2 \mathrm{mmol}), 2-(1,5,7-$ trimethyl-2-oxoindolin-3-ylidene)malononitrile (23.7 mg, $0.1 \mathrm{mmol})$, PTC C2 (9.3 mg, $0.02 \mathrm{mmol})$, 2-mercaptobenzoic acid T1 (6.2 mg, $0.04 \mathrm{mmol})$ and $\mathrm{K}_{2} \mathrm{CO}_{3}(8.3 \mathrm{mg}, 0.06 \mathrm{mmol})$ were stirred in distilled toluene $(1.0 \mathrm{~mL})$ at $\mathrm{rt}$ for 24 h. Then (E)-2-(2-nitrovinyl)thiophene (15.5 mg, $0.1 \mathrm{mmol})$ was added and the reaction was stirred at $60^{\circ} \mathrm{C}$ for $4 \mathrm{~h}$. After completion, purification by flash chromatography on silica gel $($ EtOAc/petroleum ether $=1 / 8-1 / 5)$ gave product 5s: $35.1 \mathrm{mg}$, as a white solid, yield 74\%; $[\alpha]_{\mathrm{D}}^{20}:+72.2\left(c=0.31\right.$ in $\left.\mathrm{CHCl}_{3}\right) ; 92: 8$ er, determined by HPLC analysis [Daicel Chiralpak AD-H, 
$n$-hexane $\left./ \mathrm{i}-\mathrm{PrOH}=60 / 40,1.0 \mathrm{~mL} \min ^{-1}, \lambda=254 \mathrm{~nm}\right]: \mathrm{t}($ major $)=6.20 \mathrm{~min}, \mathrm{t}($ minor $)=8.33 \mathrm{~min} ;{ }^{1} \mathrm{H}$ NMR (600 MHz, $\left.\mathrm{CDCl}_{3}\right): \delta(\mathrm{ppm}) 7.39-7.35(\mathrm{~m}, 2 \mathrm{H}), 7.31(\mathrm{~s}, 1 \mathrm{H}), 7.06(\mathrm{t}, J=8.0 \mathrm{~Hz}, 1 \mathrm{H}), 7.01(\mathrm{~s}$, $1 \mathrm{H}), 5.77(\mathrm{dd}, J=12.0,7.2 \mathrm{~Hz}, 1 \mathrm{H}), 5.67(\mathrm{~d}, J=12.0 \mathrm{~Hz}, 1 \mathrm{H}), 3.56-3.44(\mathrm{~m}, 4 \mathrm{H}), 3.26-3.24(\mathrm{~m}$, 1H), $2.90(\mathrm{~s}, 1 \mathrm{H}), 2.54-2.44(\mathrm{~m}, 4 \mathrm{H}), 2.35-2.22(\mathrm{~m}, 4 \mathrm{H}), 1.78-1.76(\mathrm{~m}, 1 \mathrm{H}) ;{ }^{13} \mathrm{C} \mathrm{NMR}(150 \mathrm{MHz}$, $\left.\mathrm{CDCl}_{3}\right): \delta(\mathrm{ppm}) 212.2,172.7,138.8,135.8,133.8,133.7,128.7,127.5,127.4,125.6,122.9,120.7$, 111.7, 110.6, 86.1, 52.6, 49.4, 48.4, 38.5, 38.2, 36.6, 30.4, 22.7, 21.1, 18.9; ESI-HRMS: calcd. for $\mathrm{C}_{25} \mathrm{H}_{22} \mathrm{~N}_{4} \mathrm{O}_{4} \mathrm{~S}+\mathrm{Na}^{+}$497.1254, found 497.1255.

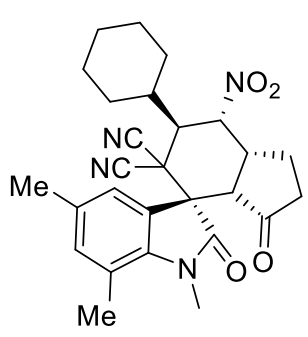

Synthesis of 5t: 2-Cyclopentenone 1a (16.4 mg, $0.2 \mathrm{mmol})$, 2-(1,5,7-trimethyl-2oxoindolin-3-ylidene)malononitrile (23.7 mg, $0.1 \mathrm{mmol})$, PTC C2 (9.3 mg, 0.02 mmol), 2-mercaptobenzoic acid T1 (6.2 mg, $0.04 \mathrm{mmol})$ and $\mathrm{K}_{2} \mathrm{CO}_{3}(8.3 \mathrm{mg}, 0.06$ mmol) were stirred in distilled toluene $(1.0 \mathrm{~mL})$ at $\mathrm{rt}$ for $24 \mathrm{~h}$. Then $(E)-(2-$ nitrovinyl)cyclohexane $(15.5 \mathrm{mg}, 0.1 \mathrm{mmol})$ was added and the reaction was stirred at $60{ }^{\circ} \mathrm{C}$ for $4 \mathrm{~h}$. After completion, purification by flash chromatography on silica gel $($ EtOAc/petroleum ether $=1 / 8-1 / 5)$ gave product 5 t: $34.1 \mathrm{mg}$, as a white solid, yield $72 \% ;[\alpha]_{\mathrm{D}}^{20}$ : $+83.4\left(c=0.43\right.$ in $\left.\mathrm{CHCl}_{3}\right)$; 92.5:7.5 er, determined by HPLC analysis [Daicel Chiralpak AD-H, $n$ hexane $\left./ i-\mathrm{PrOH}=60 / 40,1.0 \mathrm{~mL} \mathrm{~min}^{-1}, \lambda=254 \mathrm{~nm}\right]: \mathrm{t}$ (major) $=4.70 \mathrm{~min}, \mathrm{t}$ (minor) $=5.94 \mathrm{~min} ;{ }^{1} \mathrm{H}$ NMR (600 MHz, $\left.\mathrm{CDCl}_{3}\right): \delta(\mathrm{ppm}) 7.27(\mathrm{~s}, 1 \mathrm{H}), 7.01(\mathrm{~s}, 1 \mathrm{H}), 5.40(\mathrm{dd}, J=11.4,6.6 \mathrm{~Hz}, 1 \mathrm{H}), 4.01(\mathrm{~d}$, $J=11.4 \mathrm{~Hz}, 1 \mathrm{H}), 3.44(\mathrm{~s}, 3 \mathrm{H}), 3.31-3.29(\mathrm{~m}, 1 \mathrm{H}), 3.09-3.07(\mathrm{~m}, 1 \mathrm{H}), 2.81-2.79(\mathrm{~m}, 1 \mathrm{H}), 2.59$ (s, $3 \mathrm{H}), 2.43-2.35(\mathrm{~m}, 4 \mathrm{H}), 2.23-2.21(\mathrm{~m}, 1 \mathrm{H}), 1.99-1.92(\mathrm{~m}, 2 \mathrm{H}), 1.83-1.55(\mathrm{~m}, 6 \mathrm{H}), 1.31-1.14(\mathrm{~m}$, $4 \mathrm{H}) ;{ }^{13} \mathrm{C} \mathrm{NMR}\left(150 \mathrm{MHz}, \mathrm{CDCl}_{3}\right): \delta(\mathrm{ppm}) 212.5,172.5,139.0,135.7,133.5,125.8,122.8,120.5$, 112.2, 111.8, 83.9, 52.2, 48.3, 47.3, 41.8, 38.4, 38.0, 33.0, 30.3, 28.6, 26.9, 25.7, 23.0, 21.1, 19.0; ESI-HRMS: calcd. for $\mathrm{C}_{27} \mathrm{H}_{30} \mathrm{~N}_{4} \mathrm{O}_{4}+\mathrm{Na}^{+} 497.2159$, found 497.2158 .

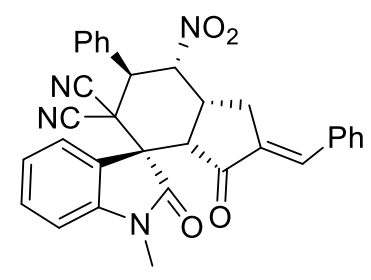

Synthesis of 7: (E)-5-benzylidenecyclopent-2-en-1-one 6 (20.4 mg, 0.12 mmol), 2-(1-methyl-2-oxoindolin-3-ylidene)malononitrile $2 \mathbf{2 a}(20.9 \mathrm{mg}, 0.1$ mmol), PTC C2 (9.3 mg, $0.02 \mathrm{mmol}), 2-$ mercaptobenzoic acid T1 (6.2 mg, $0.04 \mathrm{mmol})$ and $\mathrm{K}_{2} \mathrm{CO}_{3}(8.3 \mathrm{mg}, 0.06 \mathrm{mmol})$ were dissolved in distilled toluene $(1.0 \mathrm{~mL})$. The mixture was stirred at $25{ }^{\circ} \mathrm{C}$ for $12 \mathrm{~h}$. Then $(E)$-(2-nitrovinyl)benzene $4 \mathbf{a}(14.9$ $\mathrm{mg}, 0.1 \mathrm{mmol}$ ) was added and the reaction was moved to $50^{\circ} \mathrm{C}$ for $4 \mathrm{~h}$. After completion, purification 
by flash chromatography on silica gel (EtOAc/petroleum ether $=1 / 8-1 / 5)$ gave product 7: 48.6 mg, as a white solid, yield $92 \% ;[\alpha]_{\mathrm{D}}^{20}:+25.6\left(c=0.27\right.$ in $\left.\mathrm{CHCl}_{3}\right) ; 72: 28 \mathrm{er}$, determined by HPLC analysis [Daicel Chiralpak AD-H, $n$-hexane $/ i-\mathrm{PrOH}=60 / 40,1.0 \mathrm{~mL} \mathrm{~min}^{-1}, \lambda=254 \mathrm{~nm}$ ]: $\mathrm{t}$ (major) $=7.42 \mathrm{~min}$, $\mathrm{t}($ minor $)=8.78 \mathrm{~min} ;{ }^{1} \mathrm{H}$ NMR $\left(600 \mathrm{MHz}, \mathrm{CDCl}_{3}\right): \delta(\mathrm{ppm}) 7.78(\mathrm{~d}, J=8.4 \mathrm{~Hz}, 1 \mathrm{H}), 7.60-7.58(\mathrm{~m}$, 2H), 7.53-7.39 (m, 10H), $7.31(\mathrm{t}, J=8.4 \mathrm{~Hz}, 1 \mathrm{H}), 6.94(\mathrm{~d}, J=7.2 \mathrm{~Hz}, 1 \mathrm{H}), 5.98(\mathrm{dd}, J=12.0,7.2$ $\mathrm{Hz}, 1 \mathrm{H}), 5.60(\mathrm{~d}, J=12.0 \mathrm{~Hz}, 1 \mathrm{H}), 3.84-3.82(\mathrm{~m}, 1 \mathrm{H}), 3.55-3.53(\mathrm{~m}, 1 \mathrm{H}), 3.45-3.43$ (m, $1 \mathrm{H}), 3.19$ (s, 3H), $2.80(\mathrm{dd}, J=19.2,9.0 \mathrm{~Hz}, 1 \mathrm{H}) ;{ }^{13} \mathrm{C} \mathrm{NMR}\left(150 \mathrm{MHz}, \mathrm{CDCl}_{3}\right): \delta(\mathrm{ppm}) 199.6,171.5,143.4$, 136.9, 134.2, 132.8, 131.8, 131.4, 131.1, 130.6, 130.2, 129.6, 129.0, 124.8, 124.6, 124.4, 111.7, 110.6, 109.4, 84.0, 53.2, 49.1, 48.2, 39.9, 36.5, 29.6, 27.0; ESI-HRMS: calcd. for $\mathrm{C}_{32} \mathrm{H}_{24} \mathrm{~N}_{4} \mathrm{O}_{4}+\mathrm{Na}^{+}$551.1690, found 551.1693 .

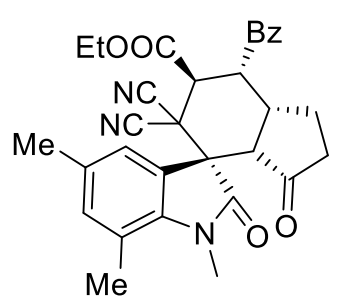

Synthesis of 8: 2-Cyclopentenone 1a (16.4 mg, $0.2 \mathrm{mmol}), 2-(1,5,7-t$ trimethyl2-oxoindolin-3-ylidene)malononitrile (23.7 mg, $0.1 \mathrm{mmol})$, PTC C2 (9.3 mg, $0.02 \mathrm{mmol})$, 2-mercaptobenzoic acid $\mathrm{T1}(6.2 \mathrm{mg}, 0.04 \mathrm{mmol})$ and $\mathrm{K}_{2} \mathrm{CO}_{3}(8.3$ $\mathrm{mg}, 0.06 \mathrm{mmol})$ were stirred in distilled toluene $(1.0 \mathrm{~mL})$ at $\mathrm{rt}$ for $24 \mathrm{~h}$. Then ethyl (E)-4-oxo-4-phenylbut-2-enoate $(20.4 \mathrm{mg}, 0.1 \mathrm{mmol})$ was added and the reaction was stirred at $60{ }^{\circ} \mathrm{C}$ for $4 \mathrm{~h}$. After completion, purification by flash chromatography on silica gel (EtOAc/petroleum ether $=1 / 8-1 / 5)$ gave product 8: $40.8 \mathrm{mg}$, as a white solid, yield $78 \% ;[\alpha]_{\mathrm{D}}^{20}:+88.5(c=0.46$ in $\mathrm{CHCl}_{3}$ ); 94:6 er, determined by HPLC analysis [Daicel Chiralpak AD-H, $n$-hexane/ $i$-PrOH $=60 / 40$,

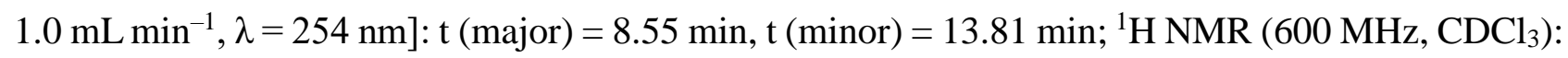
$\delta(\mathrm{ppm}) 8.06(\mathrm{~d}, J=8.4 \mathrm{~Hz}, 2 \mathrm{H}), 7.68-7.66(\mathrm{~m}, 1 \mathrm{H}), 7.56(\mathrm{t}, J=8.4 \mathrm{~Hz}, 2 \mathrm{H}), 7.36(\mathrm{~s}, 1 \mathrm{H}), 7.02(\mathrm{~s}$, 1H), 4.89-4.87 (m, 1H), 4.70-4.68 (m, 1H), 4.29-4.27 (m, 2H), 3.46 (s, 3H), 3.11-3.09 (m, 2H), 2.60-2.51 (m, 4H), $2.37(\mathrm{~s}, 3 \mathrm{H}), 2.31-2.29(\mathrm{~m}, 1 \mathrm{H}), 2.04-2.02(\mathrm{~m}, 1 \mathrm{H}), 1.45-1.43(\mathrm{~m}, 1 \mathrm{H}), 1.27(\mathrm{t}$, $J=12 \mathrm{~Hz}, 3 \mathrm{H}) ;{ }^{13} \mathrm{C} \mathrm{NMR}\left(150 \mathrm{MHz}, \mathrm{CDCl}_{3}\right): \delta(\mathrm{ppm}) 213.9,197.6,172.7,169.1,139.0,135.6,135.5$, 133.9, 133.4, 129.1, 128.2, 126.1, 123.1, 120.4, 111.3, 110.9, 62.6, 52.3, 48.2, 44.5, 44.1, 40.1, 38.6, 36.7, 30.3, 23.0, 21.1, 18.9, 13.9; ESI-HRMS: calcd. for $\mathrm{C}_{31} \mathrm{H}_{29} \mathrm{~N}_{3} \mathrm{O}_{5}+\mathrm{Na}^{+}$546.1999, found 546.1998. 


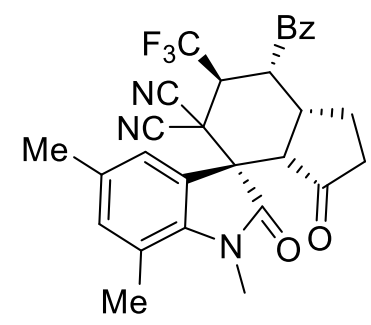

Synthesis of 9: 2-Cyclopentenone 1a $(16.4 \mathrm{mg}, 0.2 \mathrm{mmol}), 2-(1,5,7-$ trimethyl-2-oxoindolin-3-ylidene)malononitrile (23.7 mg, $0.1 \mathrm{mmol})$, PTC C2 (9.3 mg, $0.02 \mathrm{mmol}), 2$-mercaptobenzoic acid T1 (6.2 mg, $0.04 \mathrm{mmol})$ and $\mathrm{K}_{2} \mathrm{CO}_{3}(8.3 \mathrm{mg}, 0.06 \mathrm{mmol})$ were stirred in distilled toluene $(1.0 \mathrm{~mL})$ at rt for $24 \mathrm{~h}$. Then $(E)-4,4,4$-trifluoro-1-phenylbut-2-en-1-one $(20.0 \mathrm{mg}, 0.1$ mmol) was added and the reaction was stirred at $60^{\circ} \mathrm{C}$ for $4 \mathrm{~h}$. After completion, purification by flash chromatography on silica gel (EtOAc/petroleum ether $=1 / 8-1 / 5)$ gave product 9: $31.6 \mathrm{mg}$, as a white solid, yield 61\%; $[\alpha]_{\mathrm{D}}^{20}:+81.2\left(c=0.38\right.$ in $\left.\mathrm{CHCl}_{3}\right)$; 93:7 er, determined by HPLC analysis [Daicel Chiralpak AD-H, $n$-hexane $/ i$-PrOH $\left.=60 / 40,1.0 \mathrm{~mL} \mathrm{~min}^{-1}, \lambda=254 \mathrm{~nm}\right]: \mathrm{t}$ (major) $=6.06 \mathrm{~min}, \mathrm{t}$ (minor) $=7.46 \mathrm{~min} ;{ }^{1} \mathrm{H}$ NMR $\left(600 \mathrm{MHz}, \mathrm{CDCl}_{3}\right): \delta(\mathrm{ppm}) 8.06(\mathrm{~d}, J=8.4 \mathrm{~Hz}, 2 \mathrm{H}), 7.70-7.68(\mathrm{~m}, 1 \mathrm{H}), 7.58$ (t, $J=8.4 \mathrm{~Hz}, 2 \mathrm{H}), 7.40(\mathrm{~s}, 1 \mathrm{H}), 7.04(\mathrm{~s}, 1 \mathrm{H}), 5.23-5.21(\mathrm{~m}, 1 \mathrm{H}), 4.61-4.59(\mathrm{~m}, 1 \mathrm{H}), 3.47(\mathrm{~s}, 3 \mathrm{H})$, 3.16-3.14 (m, 1H), 3.05-3.03 (m, 1H), 2.67-2.65 (m, 1H), $2.56(\mathrm{~s}, 3 \mathrm{H}), 2.38(\mathrm{~s}, 3 \mathrm{H}), 2.31-2.29(\mathrm{~m}$, 1H), 2.07-2.05 (m, 1H), 1.46-1.44 (m, 1H); $\left.{ }^{13} \mathrm{C} \mathrm{NMR} \mathrm{(150} \mathrm{MHz,} \mathrm{CDCl}_{3}\right): \delta$ (ppm) 212.7, 194.3, $172.3,139.2,135.9,135.2,134.3,133.6,129.3,129.2,128.6,124.5$ (q, $\left.J_{\mathrm{CF}}=279.2 \mathrm{~Hz}\right), 122.9,111.5$, 110.5, 53.4, 52.1, 47.8, $41.7\left(\mathrm{q}, J_{\mathrm{CF}}=28.6 \mathrm{~Hz}\right), 38.2,35.9$, 30.3, 29.7, 24.3, 21.1, 19.0 ; ESI-HRMS: calcd. for $\mathrm{C}_{29} \mathrm{H}_{24} \mathrm{~F}_{3} \mathrm{~N}_{3} \mathrm{O}_{3}+\mathrm{Na}^{+}$542.1662, found 542.1665.

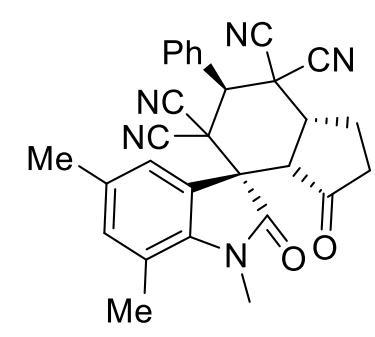

Synthesis of 10: 2-Cyclopentenone $1 \mathrm{a}(16.4 \mathrm{mg}, 0.2 \mathrm{mmol}), 2-(1,5,7-$ trimethyl-2-oxoindolin-3-ylidene)malononitrile (23.7 mg, $0.1 \mathrm{mmol})$, PTC C2 (9.3 mg, $0.02 \mathrm{mmol}), 2$-mercaptobenzoic acid T1 (6.2 mg, $0.04 \mathrm{mmol})$ and $\mathrm{K}_{2} \mathrm{CO}_{3}(8.3 \mathrm{mg}, 0.06 \mathrm{mmol})$ were stirred in distilled toluene $(1.0 \mathrm{~mL})$ at $\mathrm{rt}$ for $24 \mathrm{~h}$. Then 2-benzylidenemalononitrile $(15.4 \mathrm{mg}, 0.1 \mathrm{mmol})$ was added and the reaction was stirred at $60{ }^{\circ} \mathrm{C}$ for $4 \mathrm{~h}$. After completion, purification by flash chromatography on silica gel $($ EtOAc/petroleum ether $=1 / 8-1 / 5)$ gave product 10: $30.7 \mathrm{mg}$, as a white solid, yield $65 \% ;[\alpha]_{\mathrm{D}}^{20}:+93.4\left(c=0.52\right.$ in $\left.\mathrm{CHCl}_{3}\right) ; 94: 6 \mathrm{er}$, determined by HPLC analysis [Daicel Chiralpak AD-H, $n$-hexane $/ i$-PrOH $\left.=60 / 40,1.0 \mathrm{~mL} \mathrm{~min}^{-1}, \lambda=254 \mathrm{~nm}\right]: \mathrm{t}$ (major) $=5.35 \mathrm{~min}, \mathrm{t}($ minor $)=6.45$ min; ${ }^{1} \mathrm{H}$ NMR (600 MHz, $\left.\mathrm{CDCl}_{3}\right): \delta$ (ppm) 7.91-7.88 (m, 2H), 7.58-7.55 (m, 3H), 7.33 (s, 1H), 7.03 (s, 1H), $4.75(\mathrm{~s}, 1 \mathrm{H}), 3.96-3.94(\mathrm{~m}, 1 \mathrm{H}), 3.52(\mathrm{~s}, 3 \mathrm{H}), 3.19(\mathrm{~d}, J=18,1 \mathrm{H}), 2.60-2.54(\mathrm{~m}, 5 \mathrm{H})$, 2.37-2.29 (m, 4H), 2.19-2.17 (m, 1H); $\left.{ }^{13} \mathrm{C} \mathrm{NMR} \mathrm{(150} \mathrm{MHz,} \mathrm{CDCl}_{3}\right): \delta(\mathrm{ppm}) 205.2,172.5,135.7$, $133.5,131.3,130.2,129.9,129.8,123.5,123.2,120.8,112.2,111.9,110.8,110.3,51.3,49.9,47.2$, 
46.3, 42.0, 41.9, 35.6, 30.3, 22.7, 20.9, 19.0; ESI-HRMS: calcd. for $\mathrm{C}_{29} \mathrm{H}_{23} \mathrm{~N}_{5} \mathrm{O}_{2}+\mathrm{Na}^{+} 496.1744$, found 496.1745 .

6. More screening conditions for the cross $\mathrm{RC}$ reaction of enone 1a with $\alpha$-cyano chalcone 11a and annulation ${ }^{a}$

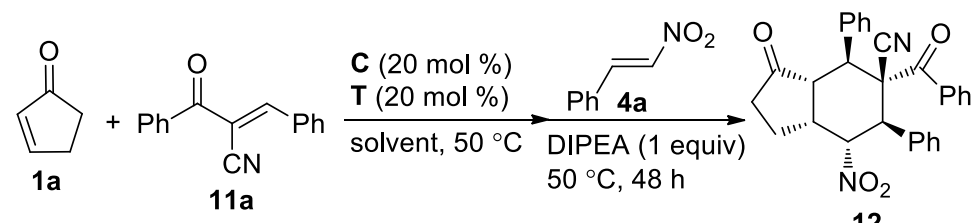

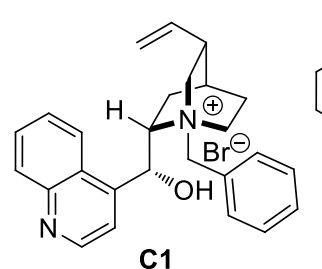

C1

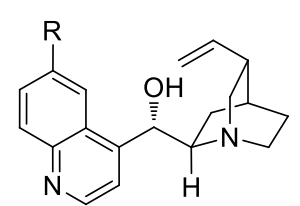

C29 R $=O \mathrm{OMe}$ C30 R = H

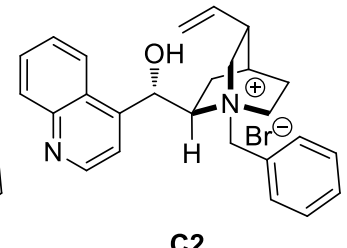

C2

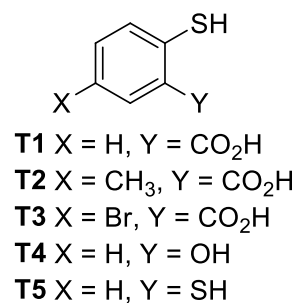

T5 $\mathrm{X}=\mathrm{H}, \mathrm{Y}=\mathrm{SH}$
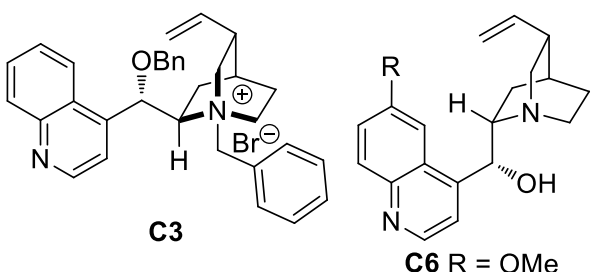

C28 R = H

\begin{tabular}{cccccc}
\hline entry & $\mathbf{C}$ & $\mathbf{T}$ & solvent & yield $(\%)^{b}$ & er $(\%)^{c}$ \\
\hline $1^{d}$ & $\mathbf{C 1}$ & $\mathbf{T 1}$ & toluene & 47 & $55.5: 44.5$ \\
$2^{d}$ & $\mathbf{C 2}$ & $\mathbf{T 1}$ & toluene & 45 & $58: 42$ \\
$3^{d}$ & $\mathbf{C 3}$ & $\mathbf{T 1}$ & toluene & 41 & $57: 43$ \\
5 & $\mathbf{C 6}$ & $\mathbf{T 1}$ & toluene & 52 & $85.5: 14.5$ \\
6 & $\mathbf{C 2 8}$ & $\mathbf{T 1}$ & toluene & 36 & $62.5: 37.5$ \\
7 & $\mathbf{C 2 9}$ & $\mathbf{T 1}$ & toluene & 42 & $63: 36$ \\
8 & $\mathbf{C 3 0}$ & $\mathbf{T 1}$ & toluene & 47 & $54.5: 45.5$ \\
9 & $\mathbf{C 6}$ & $\mathbf{T 1}$ & $o-x y l e n e$ & 79 & $89: 11$ \\
10 & $\mathbf{C 6}$ & $\mathbf{T 1}$ & $m-x y l e n e$ & 80 & $88: 12$ \\
11 & $\mathbf{C 6}$ & $\mathbf{T 1}$ & PhCF & 69 & $83.5: 16.5$ \\
12 & $\mathbf{C 6}$ & $\mathbf{T 1}$ & mesitylene & 75 & $88: 12$ \\
13 & $\mathbf{C 6}$ & $\mathbf{T 1}$ & CHCl & 62 & $78.5: 21.5$ \\
14 & $\mathbf{C 6}$ & $\mathbf{T 2}$ & $o-x y l e n e$ & 72 & $85.5: 14.5$ \\
\hline & $\mathbf{C 6}$ & $\mathbf{T 3}$ & $o-x y l e n e$ & 74 & $84.5: 15.5$ \\
\hline & & & s21 & &
\end{tabular}




\begin{tabular}{|c|c|c|c|c|c|}
\hline 15 & C6 & T4 & $o$-xylene & 64 & $74: 26$ \\
\hline 16 & C6 & T5 & $o$-xylene & 42 & $57: 43$ \\
\hline 17 & C6 & T6 & $o$-xylene & $<10$ & I \\
\hline $18^{e}$ & C6 & T7 & $o$-xylene & 51 & $51.5: 48.5$ \\
\hline $19^{d}$ & C6 & T1 & $o$-xylene & 80 & $90.5: 9.5$ \\
\hline $20^{f, g}$ & C6 & T1 & $o$-xylene & 82 & $91.5: 8.5$ \\
\hline $21^{f, g, h}$ & C6 & T1 & $o$-xylene & 82 & $91.5: 8.5$ \\
\hline $22^{f, g, h, i}$ & C6 & T1 & $o$-xylene & 77 & 89:11 \\
\hline \multicolumn{6}{|c|}{${ }^{a}$ Unless noted otherwise, reactions were performed with 2-cyclopentenone $1 \mathbf{a}(8.6 \mathrm{mg}, 0.1 \mathrm{mmol})$, } \\
\hline \multicolumn{6}{|c|}{ 11a $(11.7 \mathrm{mg}, 0.05 \mathrm{mmol}), \mathbf{C}(6.4 \mathrm{mg}, 20 \mathrm{~mol} \%)$, and thiol $\mathbf{T}(3.1 \mathrm{mg}, 20 \mathrm{~mol} \%)$ in solvent $(0.5$} \\
\hline \multicolumn{6}{|c|}{$\mathrm{mL}$ ) at $50{ }^{\circ} \mathrm{C}$ for $48-72 \mathrm{~h}$. After completion, the intermediate was obtained by flash } \\
\hline \multicolumn{6}{|c|}{ chromatography on silica gel. After that, the activated alkene $\mathbf{4 a}$ (1.0 equiv), DIPEA (1.0 equiv) } \\
\hline \multicolumn{6}{|c|}{ and $o$-xylene $(0.5 \mathrm{~mL})$ was added and the reaction was stirred at $50^{\circ} \mathrm{C}$ for $48 \mathrm{~h} .{ }^{b}$ Isolated yield for } \\
\hline \multicolumn{6}{|c|}{ two steps. ${ }^{c}$ Determined by HPLC analysis on a chiral stationary phase; $\mathrm{dr}>19: 1 .{ }^{d}$ Thiol $\mathbf{T}$ (6.4 mg, } \\
\hline \multicolumn{6}{|c|}{$40 \mathrm{~mol} \%)$ and $\mathrm{K}_{2} \mathrm{CO}_{3}(8.3 \mathrm{mg}, 60 \mathrm{~mol} \%)$ were added. ${ }^{e} \mathrm{Dr}=4: 1 .{ }^{f} \mathrm{At} 30{ }^{\circ} \mathrm{C}$ for $72 \mathrm{~h} .{ }^{g}$ With 10} \\
\hline & & & & & \\
\hline
\end{tabular}

\section{General procedure for the cross $\mathrm{RC}$ reactions of enone 1a with $\alpha$-cyano} chalcones 11 and annulations

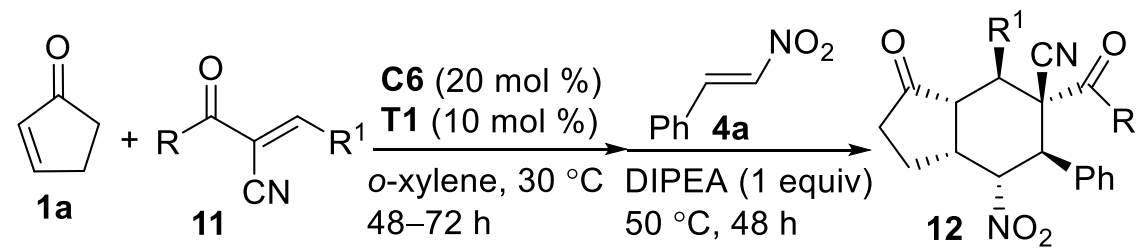

A solution of 2-cyclopentenone $\mathbf{1 a}(0.2 \mathrm{mmol}, 2.0$ equiv), $\alpha$-cyano chalcone $\mathbf{1 1}(0.1 \mathrm{mmol}, 1.0$ equiv), quinine C6 (20 mol \%), thiol T1 $(10 \mathrm{~mol} \%)$ in $o$-xylene $(1.0 \mathrm{~mL})$ was stirred at $30{ }^{\circ} \mathrm{C}$ for 48-72 h. The reaction was monitored by TLC. After completion, the intermediate was obtained by flash chromatography on silica gel (EtOAc/petroleum ether $=1 / 12-1 / 9)$. Then, the activated alkene $4 \mathbf{a}$ (0.1 mmol, 1.0 equiv), $N, N$-Diisopropylenthylamine (DIPEA, $0.1 \mathrm{mmol}, 1.0$ equiv) and $o$-xylene $(1.0 \mathrm{~mL})$ were added and the reaction was stirred at $50{ }^{\circ} \mathrm{C}$ for $48 \mathrm{~h}$ (monitored by TLC). After completion, the product $\mathbf{1 2}$ was obtained by flash chromatography on silica gel (EtOAc/petroleum 
ether $=1 / 9-1 / 7)$.

The racemates could not be obtained by using DABCO as the catalyst. So two peaks of these enantiomers were assigned by HPLC analysis on a chiral stationary phase with the mixture of two enantiomers, which were produced by using quinine and cinchonine as the catalyst, respectively.

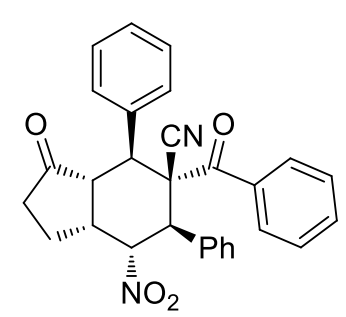

Synthesis of 12a: A solution of 2-cyclopentenone 1a (16.4 mg, $0.2 \mathrm{mmol}),(E)-$ 2-benzoyl-3-phenylacrylonitrile 11a (23.3 mg, $0.1 \mathrm{mmol})$, quinine C6 (6.4 mg, $0.02 \mathrm{mmol})$, thiol T1 $(1.5 \mathrm{mg}, 0.01 \mathrm{mmol})$ in $o$-xylene $(1.0 \mathrm{~mL})$ was stirred at $30{ }^{\circ} \mathrm{C}$ for $72 \mathrm{~h}$. The reaction was monitored by TLC. After completion, the intermediate was obtained by flash chromatography on silica gel $($ EtOAc/petroleum ether =1/12-1/9). After that, the activated alkene 4a $(14.9 \mathrm{mg}, 0.1 \mathrm{mmol})$, DIPEA $(12.9 \mathrm{mg}, 0.1 \mathrm{mmol})$ and $o$-xylene $(1.0 \mathrm{~mL})$ was added and the reaction were stirred at $50{ }^{\circ} \mathrm{C}$ for 48 h. After completion, purification by flash chromatography on silica gel $($ EtOAc/petroleum ether $=$ 1/9-1/7) gave product 12a: $39.0 \mathrm{mg}$, as a white solid, yield $84 \%$; $[\alpha]_{\mathrm{D}}{ }^{25}=+58.8\left(c=0.32\right.$ in $\left.\mathrm{CHCl}_{3}\right)$; 91.5:8.5 er, determined by HPLC analysis [Daicel chiralcel OD-H, $n$-hexane $/ i-\mathrm{PrOH}=60 / 40,1.0 \mathrm{~mL}$ $\left.\min ^{-1}, \lambda=254 \mathrm{~nm}\right]: \mathrm{t}$ (minor) $=13.77 \mathrm{~min}, \mathrm{t}$ (major) $\left.=26.67 \mathrm{~min}\right] ;{ }^{1} \mathrm{H}$ NMR $\left(400 \mathrm{MHz}, \mathrm{CDCl}_{3}\right): \delta$ (ppm) 7.43-7.42 (m, 2H), 7.33-7.26 (m, 8H), $7.20(\mathrm{t}, J=7.6 \mathrm{~Hz}, 1 \mathrm{H}), 6.97-6.94(\mathrm{~m}, 2 \mathrm{H}), 6.31(\mathrm{~d}, J$ $=7.6 \mathrm{~Hz}, 2 \mathrm{H}), 5.92(\mathrm{dd}, J=12.8,5.6 \mathrm{~Hz}, 1 \mathrm{H}), 4.45(\mathrm{~d}, J=8.8 \mathrm{~Hz}, 1 \mathrm{H}), 3.75-3.72(\mathrm{~m}, 1 \mathrm{H}), 3.45-3.37$ (m, 2H), 2.69-2.51 (m, 2H), 2.41-2.32 (m, 1H), 2.03-1.96 (m, 1H); $\left.{ }^{13} \mathrm{C} \mathrm{NMR} \mathrm{(100} \mathrm{MHz,} \mathrm{CDCl}_{3}\right): \delta$ (ppm) 211.6, 196.4, 137.0, 133.2, 132.7, 132.1, 129.3, 129.2, 129.0, 128.9, 127.5, 126.6, 118.0, 85.4, 62.0, 49.5, 47.4, 46.9, 39.5, 36.4, 21.1; ESI-HRMS: calcd. for $\mathrm{C}_{29} \mathrm{H}_{24} \mathrm{~N}_{2} \mathrm{O}_{4}+\mathrm{Na}^{+} 487.1628$, found 487.1630.

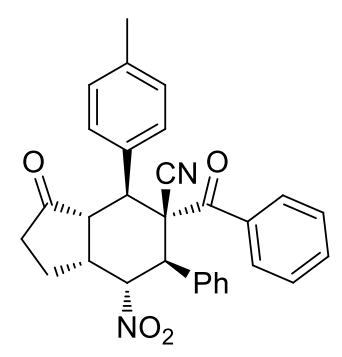

Synthesis of 12b: A solution of 2-cyclopentenone 1a (16.4 mg, $0.2 \mathrm{mmol}),(E)$ 2-benzoyl-3-(p-tolyl)acrylonitrile (24.7 mg, $0.1 \mathrm{mmol})$, quinine C6 (6.4 mg, $0.02 \mathrm{mmol})$, thiol $\mathbf{T 1}(1.5 \mathrm{mg}, 0.01 \mathrm{mmol})$ was stirred at $30^{\circ} \mathrm{C}$ for $72 \mathrm{~h}$. The reaction was monitored by TLC. After completion, the intermediate was obtained by flash chromatography on silica gel (EtOAc/petroleum ether $=1 / 12$ 1/9). After that, the activated alkene $4 \mathbf{a}(14.9 \mathrm{mg}, 0.1 \mathrm{mmol})$, DIPEA (12.9 $\mathrm{mg}, 0.1 \mathrm{mmol})$ and $o$ xylene $(1.0 \mathrm{~mL})$ were added and the reaction was stirred at $50{ }^{\circ} \mathrm{C}$ for $48 \mathrm{~h}$. After completion, 
purification by flash chromatography on silica gel (EtOAc/petroleum ether $=1 / 9-1 / 7)$ gave product 12b: $37.3 \mathrm{mg}$, as a white solid, yield $78 \% ;[\alpha]_{\mathrm{D}}^{25}=+90.0\left(c=0.24\right.$ in $\left.\mathrm{CHCl}_{3}\right) ; 90.5: 9.5 \mathrm{er}$, determined by HPLC analysis [Daicel chiralcel OD-H, $n$-hexane/ $i-\mathrm{PrOH}=60 / 40,1.0 \mathrm{~mL} \mathrm{m^{-1 }}, \lambda=254 \mathrm{~nm}$ ]: $\mathrm{t}$ $($ minor $)=14.23 \mathrm{~min}, \mathrm{t}($ major $)=40.04 \mathrm{~min}] ;{ }^{1} \mathrm{H}$ NMR $\left(400 \mathrm{MHz}, \mathrm{CDCl}_{3}\right): \delta(\mathrm{ppm}) 7.42-7.41(\mathrm{~m}$, 2H), 7.31-7.18 (m, 6H), 7.10-7.08 (m, 2H), 6.98-6.94 (m, 2H), 6.33 (d, J = 8.0 Hz, 2H), 5.92 (dd, $J$ $=12.4,5.2 \mathrm{~Hz}, 1 \mathrm{H}), 4.44(\mathrm{~d}, J=12.4 \mathrm{~Hz}, 1 \mathrm{H}), 3.72-3.69(\mathrm{~m}, 1 \mathrm{H}), 3.42-3.34(\mathrm{~m}, 2 \mathrm{H}), 2.68-2.50(\mathrm{~m}$, 2H), 2.39-2.30 (m, 1H), $2.26(\mathrm{~s}, 3 \mathrm{H}), 2.01-1.94(\mathrm{~m}, 1 \mathrm{H}) ;{ }^{13} \mathrm{C} \mathrm{NMR}\left(100 \mathrm{MHz}, \mathrm{CDCl}_{3}\right): \delta(\mathrm{ppm})$ 211.6, 196.4, 138.7, 137.0, 132.7, 132.0, 130.1, 129.6, 129.3, 129.2, 127.5, 126.6, 118.1, 85.5, 62.1, 49.6, 47.4, 46.5, 39.6, 36.3, 21.1, 21.0; ESI-HRMS: calcd. for $\mathrm{C}_{30} \mathrm{H}_{26} \mathrm{~N}_{2} \mathrm{O}_{4}+\mathrm{Na}^{+}$501.1785, found 501.1786.

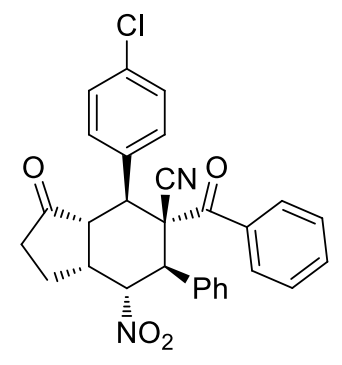

Synthesis of 12c: A solution of 2-cyclopentenone 1a (16.4 mg, $0.2 \mathrm{mmol}),(E)-$ 2-benzoyl-3-(4-chlorophenyl)acrylonitrile (26.7 mg, $0.1 \mathrm{mmol})$, quinine C6 (6.4 mg, $0.02 \mathrm{mmol})$, thiol T1 $(1.5 \mathrm{mg}, 0.01 \mathrm{mmol})$ in $o$-xylene $(1.0 \mathrm{~mL})$ was stirred at $30^{\circ} \mathrm{C}$ for $72 \mathrm{~h}$. The reaction was monitored by TLC. After completion, the intermediate was obtained by flash chromatography on silica gel $($ EtOAc/petroleum ether $=1 / 12-1 / 9)$. After that, the activated alkene 4a $(14.9 \mathrm{mg}, 0.1 \mathrm{mmol})$, DIPEA $(12.9 \mathrm{mg}, 0.1 \mathrm{mmol})$ and $o$-xylene $(1.0 \mathrm{~mL})$ were added and the reaction was stirred at $50{ }^{\circ} \mathrm{C}$ for 48 h. After completion, purification by flash chromatography on silica gel (EtOAc/petroleum ether $=$ 1/9-1/7) gave product 12c: $38.8 \mathrm{mg}$, as a white solid, yield $78 \%$; $[\alpha]_{\mathrm{D}}^{25}=+72.8\left(c=0.5\right.$ in $\left.\mathrm{CHCl}_{3}\right)$; 90:10 er, determined by HPLC analysis [Daicel chiralcel OD-H, $n$-hexane/i-PrOH =60/40, $1.0 \mathrm{~mL}$ $\left.\min ^{-1}, \lambda=254 \mathrm{~nm}\right]: \mathrm{t}$ (minor) $=13.39 \mathrm{~min}, \mathrm{t}$ (major) $\left.=39.98 \mathrm{~min}\right] ;{ }^{1} \mathrm{H} \mathrm{NMR}\left(400 \mathrm{MHz}, \mathrm{CDCl}_{3}\right): \delta$ (ppm) 7.42-7.40 (m, 2H), 7.33-7.23 (m, 8H), $7.01(\mathrm{t}, J=8.0 \mathrm{~Hz}, 2 \mathrm{H}), 6.41(\mathrm{~d}, J=7.6 \mathrm{~Hz}, 2 \mathrm{H}), 5.91$ $(\mathrm{dd}, J=12.8,6.0 \mathrm{~Hz}, 1 \mathrm{H}), 4.40(\mathrm{~d}, J=12.8 \mathrm{~Hz}, 1 \mathrm{H}), 3.74-3.71(\mathrm{~m}, 1 \mathrm{H}), 3.45-3.32(\mathrm{~m}, 2 \mathrm{H}), 2.69-2.48$ (m, 2H), 2.42-2.32 (m, 1H), 2.03-1.98 (m, 1H); $\left.{ }^{13} \mathrm{C} \mathrm{NMR} \mathrm{(100} \mathrm{MHz,} \mathrm{CDCl}_{3}\right): \delta(\mathrm{ppm}) 211.5,196.0$, 136.7, 135.0, 132.6, 132.5, 131.8, 131.0, 129.4, 129.1, 129.0, 127.7, 127.7, 126.8, 117.9, 85.3, 61.7, 49.4, 47.4, 46.2, 39.5, 36.4, 21.1; ESI-HRMS: calcd. for $\mathrm{C}_{29} \mathrm{H}_{23}{ }^{35} \mathrm{ClN}_{2} \mathrm{O}_{4}+\mathrm{Na}^{+} 521.1239$, found 521.1240, $\mathrm{C}_{29} \mathrm{H}_{23}{ }^{37} \mathrm{ClN}_{2} \mathrm{O}_{4}+\mathrm{Na}^{+}$523.1215, found 523.1217. 


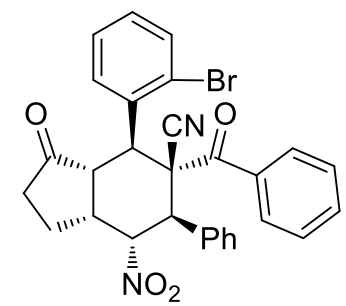

Synthesis of 12d: A solution of 2-cyclopentenone 1a (16.4 mg, $0.2 \mathrm{mmol}),(E)$ 2-benzoyl-3-(2-bromophenyl)acrylonitrile $(31.0 \mathrm{mg}, 0.1 \mathrm{mmol})$, quinine C6 (6.4 mg, $0.02 \mathrm{mmol})$, thiol T1 $(1.5 \mathrm{mg}, 0.01 \mathrm{mmol})$ in $o$-xylene $(1.0 \mathrm{~mL})$ was stirred at $30{ }^{\circ} \mathrm{C}$ for $72 \mathrm{~h}$. The reaction was monitored by TLC. After completion, the intermediate was obtained by flash chromatography on silica gel $($ EtOAc/petroleum ether $=1 / 12-1 / 9)$. After that, the activated alkene 4a $(14.9 \mathrm{mg}, 0.1 \mathrm{mmol})$, DIPEA $(12.9 \mathrm{mg}, 0.1 \mathrm{mmol})$ and $o$-xylene $(1.0 \mathrm{~mL})$ were added and the reaction was stirred at $50{ }^{\circ} \mathrm{C}$ for 48 h. After completion, purification by flash chromatography on silica gel (EtOAc/petroleum ether $=$ 1/9-1/7) gave product 12d: $40.1 \mathrm{mg}$, as a white solid, yield $74 \%$; $[\alpha]_{\mathrm{D}}{ }^{25}=+105.7\left(c=0.14\right.$ in $\left.\mathrm{CHCl}_{3}\right)$; 92:8 er, determined by HPLC analysis [Daicel chiralcel OD-H, $n$-hexane/i-PrOH = 80/20, $1.0 \mathrm{~mL}$ $\left.\min ^{-1}, \lambda=254 \mathrm{~nm}\right]: \mathrm{t}($ minor $)=26.49 \mathrm{~min}, \mathrm{t}($ major $\left.)=33.03 \mathrm{~min}\right] ;{ }^{1} \mathrm{H} \mathrm{NMR}\left(400 \mathrm{MHz}, \mathrm{CDCl}_{3}\right): \delta$ (ppm) 7.89-7.85 (m, 1H), 7.55-7.47 (m, 3H), 7.36-7.29 (m, 3H), 7.25-7.20 (m, 2H), 7.13-7.09 (m, 1H), 7.02-6.98 (m, 2H), $6.43(\mathrm{~d}, J=7.6 \mathrm{~Hz}, 2 \mathrm{H}), 5.95(\mathrm{dd}, J=12.4,6.0 \mathrm{~Hz}, 1 \mathrm{H}), 4.59$ (dd, $J=26.0$, 13.2 Hz, 2H), 3.43-3.35 (m, 1H), $3.14(\mathrm{dd}, J=13.2 \mathrm{~Hz}, J=6.4 \mathrm{~Hz}, 1 \mathrm{H}), 2.86-2.79(\mathrm{~m}, 1 \mathrm{H})$, 2.72-2.60 (m, 1H), 2.41-2.31 (m, 1H), 2.07-2.00 (m, 1H); $\left.{ }^{13} \mathrm{C} \mathrm{NMR} \mathrm{(100} \mathrm{MHz,} \mathrm{CDCl}_{3}\right): \delta(\mathrm{ppm})$ 211.4, 194.6, 136.9, 133.5, 133.4, 132.6, 132.2, 130.2, 129.4, 129.1, 129.0, 128.1, 127.7, 126.6, 118.7, 85.4, 60.4, 51.6, 48.0, 44.2, 39.8, 36.5, 21.4; ESI-HRMS: calcd. for $\mathrm{C}_{29} \mathrm{H}_{23}{ }^{79} \mathrm{BrN}_{2} \mathrm{O}_{4}+\mathrm{Na}^{+}$565.0733, found 565.0737, $\mathrm{C}_{29} \mathrm{H}_{23}{ }^{81} \mathrm{BrN}_{2} \mathrm{O}_{4}+\mathrm{Na}^{+}$567.0713, found 567.0723.

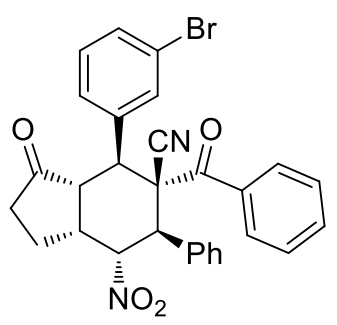

Synthesis of 12e: A solution of 2-cyclopentenone 1a (16.4 mg, $0.2 \mathrm{mmol}),(E)$ 2-benzoyl-3-(3-bromophenyl)acrylonitrile (31.0 mg, $0.1 \mathrm{mmol})$, quinine C6 $(6.4 \mathrm{mg}, 0.02 \mathrm{mmol})$, thiol T1 $(1.5 \mathrm{mg}, 0.01 \mathrm{mmol})$ in $o$-xylene $(1.0 \mathrm{~mL})$ was stirred at $30^{\circ} \mathrm{C}$ for $48 \mathrm{~h}$. The reaction was monitored by TLC. After completion, the intermediate was obtained by flash chromatography on silica gel $($ EtOAc/petroleum ether $=1 / 12-1 / 9)$. After that, the activated alkene 4a $(14.9 \mathrm{mg}, 0.1 \mathrm{mmol})$, DIPEA $(12.9 \mathrm{mg}, 0.1 \mathrm{mmol})$ and $o$-xylene $(1.0 \mathrm{~mL})$ were added and the reaction was stirred at $50{ }^{\circ} \mathrm{C}$ for 48 h. After completion, purification by flash chromatography on silica gel (EtOAc/petroleum ether $=$ 1/9-1/7) gave product 12e: $43.9 \mathrm{mg}$, as a white solid, yield $81 \% ;[\alpha]_{\mathrm{D}}^{25}=+85.0\left(c=0.28\right.$ in $\left.\mathrm{CHCl}_{3}\right)$; 90:10 er, determined by HPLC analysis [Daicel chiralcel OD-H, $n$-hexane/i-PrOH =60/40, $1.0 \mathrm{~mL}$ $\left.\min ^{-1}, \lambda=254 \mathrm{~nm}\right]: \mathrm{t}$ (minor) $=14.70 \mathrm{~min}, \mathrm{t}$ (major) $\left.=28.45 \mathrm{~min}\right] ;{ }^{1} \mathrm{H} \mathrm{NMR}\left(400 \mathrm{MHz}, \mathrm{CDCl}_{3}\right): \delta$ 
(ppm) 7.43-7.23 (m, 9H), $7.16(\mathrm{t}, J=8.0 \mathrm{~Hz}, 1 \mathrm{H}), 7.01(\mathrm{t}, J=8.0 \mathrm{~Hz}, 2 \mathrm{H}), 6.45(\mathrm{~d}, J=7.6 \mathrm{~Hz}, 2 \mathrm{H})$, $5.91(\mathrm{dd}, J=12.8,6.0 \mathrm{~Hz}, 1 \mathrm{H}), 4.40(\mathrm{~d}, J=12.8 \mathrm{~Hz}, 1 \mathrm{H}), 3.70-3.66(\mathrm{~m}, 1 \mathrm{H}), 3.45-3.32(\mathrm{~m}, 2 \mathrm{H})$, $2.67(\mathrm{dd}, J=18.8,8.8 \mathrm{~Hz}, 1 \mathrm{H}), 2.58-2.47(\mathrm{~m}, 1 \mathrm{H}), 2.42-2.33(\mathrm{~m}, 1 \mathrm{H}), 2.04-2.00(\mathrm{~m}, 1 \mathrm{H}) ;{ }^{13} \mathrm{C} \mathrm{NMR}$ $\left(100 \mathrm{MHz}, \mathrm{CDCl}_{3}\right): \delta$ (ppm) 211.3, 195.9, 136.7, 133.5, 132.5, 132.1, 130.3, 129.4, 129.0, 127.7, 126.9, 122.8, 117.8, 85.2, 61.7, 49.2, 47.4, 46.4, 39.5, 36.5, 21.1; ESI-HRMS: calcd. for $\mathrm{C}_{29} \mathrm{H}_{23}{ }^{79} \mathrm{BrN}_{2} \mathrm{O}_{4}+\mathrm{Na}^{+}$565.0733, found 565.0733, $\mathrm{C}_{29} \mathrm{H}_{23}{ }^{81} \mathrm{BrN}_{2} \mathrm{O}_{4}+\mathrm{Na}^{+}$567.0713, found 567.0718.

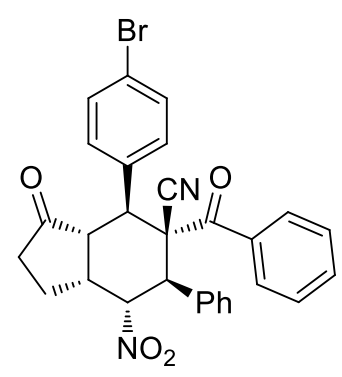

Synthesis of 12f: A solution of 2-cyclopentenone $1 \mathrm{a}(16.4 \mathrm{mg}, 0.2 \mathrm{mmol}),(E)$ 2-benzoyl-3-(4-bromophenyl)acrylonitrile (31.0 mg, $0.1 \mathrm{mmol})$, quinine C6 $(6.4 \mathrm{mg}, 0.02 \mathrm{mmol})$, thiol T1 $(1.5 \mathrm{mg}, 0.01 \mathrm{mmol})$ in $o$-xylene $(1.0 \mathrm{~mL})$ was stirred at $30{ }^{\circ} \mathrm{C}$ for $48 \mathrm{~h}$. The reaction was monitored by TLC. After completion, the intermediate was obtained by flash chromatography on silica gel $($ EtOAc/petroleum ether $=1 / 12-1 / 9)$. After that, the activated alkene 4a $(14.9 \mathrm{mg}, 0.1 \mathrm{mmol})$, DIPEA $(12.9 \mathrm{mg}, 0.1 \mathrm{mmol})$ and $o$-xylene $(1.0 \mathrm{~mL})$ were added and the reaction was stirred at $50{ }^{\circ} \mathrm{C}$ for 48 h. After completion, purification by flash chromatography on silica gel (EtOAc/petroleum ether $=$ 1/9-1/7) gave product 12f: $42.8 \mathrm{mg}$, as a white solid, yield $79 \%$; $[\alpha]_{\mathrm{D}}^{25}=+92.0\left(c=0.15\right.$ in $\left.\mathrm{CHCl}_{3}\right)$; 96.5:3.5 er, determined by HPLC analysis [Daicel chiralcel OD-H, $n$-hexane $/ i$-PrOH = 60/40, $1.0 \mathrm{~mL}$ $\left.\min ^{-1}, \lambda=254 \mathrm{~nm}\right]: \mathrm{t}$ (minor) $=13.98 \mathrm{~min}, \mathrm{t}$ (major) $\left.=49.31 \mathrm{~min}\right] ;{ }^{1} \mathrm{H} \mathrm{NMR}\left(400 \mathrm{MHz}, \mathrm{CDCl}_{3}\right): \delta$ (ppm) 7.43-7.41 (m, 4H), 7.33-7.21 (m, 6H), $7.01(\mathrm{t}, J=8.0 \mathrm{~Hz}, 2 \mathrm{H}), 6.41(\mathrm{~d}, J=8.0 \mathrm{~Hz}, 2 \mathrm{H}), 5.91$ (dd, $J=12.4,5.6 \mathrm{~Hz}, 1 \mathrm{H}), 4.40(\mathrm{~d}, J=12.4 \mathrm{~Hz}, 1 \mathrm{H}), 3.72-3.69(\mathrm{~m}, 1 \mathrm{H}), 3.45-3.32(\mathrm{~m}, 2 \mathrm{H}), 2.69-2.48$ (m, 2H), 2.42-2.33 (m, 1H), 2.04-1.97 (m, 1H); $\left.{ }^{13} \mathrm{C} \mathrm{NMR} \mathrm{(100} \mathrm{MHz,} \mathrm{CDCl}_{3}\right): \delta(\mathrm{ppm}) 211.5,196.0$, $136.7,132.5,132.3,132.1,131.3,129.4,129.4,129.0,127.7,126.8,123.2,117.9$, 85.2, 61.6, 49.3, 47.4, 46.3, 39.5, 36.5, 21.1; ESI-HRMS: calcd. for $\mathrm{C}_{29} \mathrm{H}_{23}{ }^{79} \mathrm{BrN}_{2} \mathrm{O}_{4}+\mathrm{Na}^{+}$565.0733, found 565.0731, $\mathrm{C}_{29} \mathrm{H}_{23}{ }^{81} \mathrm{BrN}_{2} \mathrm{O}_{4}+\mathrm{Na}^{+}$567.0713, found 567.0717.

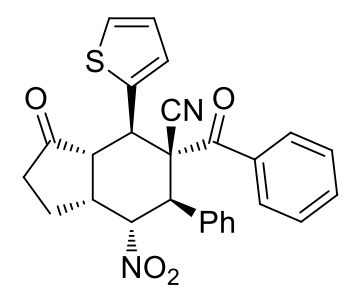

Synthesis of 12g: A solution of 2-cyclopentenone $1 \mathrm{a}(16.4 \mathrm{mg}, 0.2 \mathrm{mmol}),(E)$ 2-benzoyl-3-(thiophen-2-yl)acrylonitrile (23.9 mg, $0.1 \mathrm{mmol})$, quinine C6 (6.4 $\mathrm{mg}, 0.02 \mathrm{mmol})$, thiol T1 $(1.5 \mathrm{mg}, 0.01 \mathrm{mmol})$ in $o$-xylene $(1.0 \mathrm{~mL})$ was stirred at $30{ }^{\circ} \mathrm{C}$ for $72 \mathrm{~h}$. The reaction was monitored by TLC. After completion, the intermediate was obtained by flash chromatography on silica gel $($ EtOAc/petroleum ether $=1 / 12-$ 
1/9). After that, the activated alkene $4 \mathbf{a}(14.9 \mathrm{mg}, 0.1 \mathrm{mmol})$, DIPEA (12.9 $\mathrm{mg}, 0.1 \mathrm{mmol})$ and $o$ xylene $(1.0 \mathrm{~mL})$ were added and the reaction was stirred at $50{ }^{\circ} \mathrm{C}$ for $48 \mathrm{~h}$. After completion, purification by flash chromatography on silica gel (EtOAc/petroleum ether $=1 / 9-1 / 7)$ gave product 12g: $37.1 \mathrm{mg}$, as a white solid, yield 79\%; $[\alpha]_{\mathrm{D}}{ }^{25}=+35.2\left(c=0.75\right.$ in $\left.\mathrm{CHCl}_{3}\right) ; 91.5: 8.5 \mathrm{er}$, determined by HPLC analysis [Daicel chiralcel OD-H, $n$-hexane/ $i$-PrOH $=60 / 40,1.0 \mathrm{~mL} \mathrm{~min}^{-1}, \lambda=254 \mathrm{~nm}$ ]: $\mathrm{t}$ $($ minor $)=18.02 \mathrm{~min}, \mathrm{t}($ major $)=53.62 \mathrm{~min}] ;{ }^{1} \mathrm{H} \mathrm{NMR}\left(400 \mathrm{MHz}, \mathrm{CDCl}_{3}\right): \delta(\mathrm{ppm}) 7.42-7.40(\mathrm{~m}$, 2H), 7.31-7.21 (m, 5H), 7.06-7.01 (m, 3H), $6.91(\mathrm{dd}, J=5.2 \mathrm{~Hz}, J=3.6 \mathrm{~Hz}, 1 \mathrm{H}), 6.51(\mathrm{~d}, J=7.2$ $\mathrm{Hz}, 2 \mathrm{H}), 5.90(\mathrm{dd}, J=12.8,6.0 \mathrm{~Hz}, 1 \mathrm{H}), 4.40(\mathrm{~d}, J=12.4 \mathrm{~Hz}, 1 \mathrm{H}), 4.01(\mathrm{~d}, J=13.2 \mathrm{~Hz}, 1 \mathrm{H})$, 3.41-3.27 (m, 2H), 2.72-2.65 (m, 1H), 2.57-2.46 (m, 1H), 2.41-2.33 (m, 1H), 2.02-1.95 (m, 1H); ${ }^{13} \mathrm{C}$ NMR (100 MHz, $\mathrm{CDCl}_{3}$ ): $\delta$ (ppm) 210.9, 196.3, 136.9, 135.5, 132.6, 132.4, 129.4, 129.3, 128.5, 127.7, 127.0, 126.8, 126.3, 117.9, 85.2, 62.5, 51.4, 47.2, 42.7, 39.7, 36.3, 21.0; ESI-HRMS: calcd. for $\mathrm{C}_{27} \mathrm{H}_{22} \mathrm{~N}_{2} \mathrm{O}_{4} \mathrm{~S}+\mathrm{Na}^{+} 493.1192$, found 493.1188 .

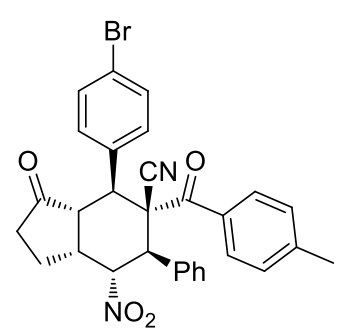

Synthesis of 12h: A solution of 2-cyclopentenone 1a (16.4 mg, $0.2 \mathrm{mmol}),(E)$ 3-(4-bromophenyl)-2-(4-methylbenzoyl)acrylonitrile (32.5 mg, $0.1 \mathrm{mmol}$ ), quinine C6 (6.4 mg, $0.02 \mathrm{mmol})$, thiol T1 (1.5 mg, $0.01 \mathrm{mmol})$ in $o$-xylene (1.0 $\mathrm{mL}$ ) was stirred at $30^{\circ} \mathrm{C}$ for $72 \mathrm{~h}$. The reaction was monitored by TLC. After completion, the intermediate was obtained by flash chromatography on silica gel $($ EtOAc/petroleum ether $=1 / 12-1 / 9)$. After that, the activated alkene 4a $(14.9 \mathrm{mg}, 0.1 \mathrm{mmol})$, DIPEA $(12.9 \mathrm{mg}, 0.1 \mathrm{mmol})$ and $o$-xylene $(1.0 \mathrm{~mL})$ were added and the reaction was stirred at $50{ }^{\circ} \mathrm{C}$ for $48 \mathrm{~h}$. After completion, purification by flash chromatography on silica gel (EtOAc/petroleum ether $=1 / 9-1 / 7)$ gave product $\mathbf{1 2 h}: 45.6 \mathrm{mg}$, as a white solid, yield $82 \% ;[\alpha]_{\mathrm{D}}{ }^{25}=+85.0(c=0.24$ in $\mathrm{CHCl}_{3}$ ); 96:4 er, determined by HPLC analysis [Daicel chiralcel OD-H, $n$-hexane/i-PrOH $=60 / 40$, $\left.1.0 \mathrm{~mL} \mathrm{~min}{ }^{-1}, \lambda=254 \mathrm{~nm}\right]: \mathrm{t}$ (minor) $=13.56 \mathrm{~min}, \mathrm{t}$ (major) $\left.=46.91 \mathrm{~min}\right] ;{ }^{1} \mathrm{H} \mathrm{NMR}(400 \mathrm{MHz}$, $\left.\mathrm{CDCl}_{3}\right): \delta(\mathrm{ppm}) 7.42-7.39(\mathrm{~m}, 4 \mathrm{H}), 7.31-7.20(\mathrm{~m}, 5 \mathrm{H}), 6.81(\mathrm{~d}, J=8.0 \mathrm{~Hz}, 2 \mathrm{H}), 6.40(\mathrm{~d}, J=8.0 \mathrm{~Hz}$, 2H), $5.91(\mathrm{dd}, J=12.8,6.0 \mathrm{~Hz}, 1 \mathrm{H}), 4.40(\mathrm{~d}, J=12.8 \mathrm{~Hz}, 1 \mathrm{H}), 3.72-3.69(\mathrm{~m}, 1 \mathrm{H}), 3.44-3.31(\mathrm{~m}$, 2H), 2.68-2.48 (m, 2H), 2.42-2.32 (m, 1H), $2.19(\mathrm{~s}, 3 \mathrm{H}), 2.03-1.96(\mathrm{~m}, 1 \mathrm{H}) ;{ }^{13} \mathrm{C}$ NMR (100 MHz, $\left.\mathrm{CDCl}_{3}\right): \delta$ (ppm) 211.6, 195.3, 143.7, 134.1, 132.6, 132.3, 132.0, 131.2, 129.4, 129.3, 129.0, 128.5, 127.1, 123.1, 118.0, 85.2, 61.4, 49.3, 47.3, 46.1, 39.5, 36.5, 21.5, 21.1; ESI-HRMS: calcd. for $\mathrm{C}_{30} \mathrm{H}_{25}{ }^{79} \mathrm{BrN}_{2} \mathrm{O}_{4}+\mathrm{Na}^{+} 579.0890$, found 579.0891, $\mathrm{C}_{30} \mathrm{H}_{25}{ }^{81} \mathrm{BrN}_{2} \mathrm{O}_{4}+\mathrm{Na}^{+}$581.0869, found 581.0873. 
<smiles>N#C[C@]1(C(=O)c2cccc(Br)c2)[C@H](c2ccccc2)[C@H]([N+](=O)[O-])[C@H]2CCC(=O)[C@H]21</smiles>

$($ EtOAc/petroleum ether $=1 / 12-1 / 9)$. After that, the activated alkene 4a $(14.9 \mathrm{mg}, 0.1 \mathrm{mmol})$, DIPEA $(12.9 \mathrm{mg}, 0.1 \mathrm{mmol})$ and $o$-xylene $(1.0 \mathrm{~mL})$ were added and the reaction was stirred at $50{ }^{\circ} \mathrm{C}$ for 48 h. After completion, purification by flash chromatography on silica gel (EtOAc/petroleum ether $=$ 1/9-1/7) gave product 12i: $45.0 \mathrm{mg}$, as a white solid, yield $83 \% ; 17: 1 \mathrm{dr},[\alpha]_{\mathrm{D}}{ }^{25}=+55.5(c=0.22 \mathrm{in}$ $\mathrm{CHCl}_{3}$ ); 95:5 er, determined by HPLC analysis [Daicel chiralcel OD-H, $n$-hexane/i-PrOH = 80/20, $\left.1.0 \mathrm{~mL} \min ^{-1}, \lambda=254 \mathrm{~nm}\right]: \mathrm{t}$ (minor) $=27.17 \mathrm{~min}, \mathrm{t}$ (major) $\left.=54.73 \mathrm{~min}\right] ;{ }^{1} \mathrm{H} \mathrm{NMR}(400 \mathrm{MHz}$, $\left.\mathrm{CDCl}_{3}\right): \delta(\mathrm{ppm}) 7.41-7.33(\mathrm{~m}, 11 \mathrm{H}), 6.82(\mathrm{t}, J=8.0 \mathrm{~Hz}, 1 \mathrm{H}), 6.23(\mathrm{~s}, 1 \mathrm{H}), 6.09(\mathrm{~d}, J=7.6 \mathrm{~Hz}, 1 \mathrm{H})$, $5.90(\mathrm{dd}, J=12.4,5.2 \mathrm{~Hz}, 1 \mathrm{H}), 4.41$ (d, $J=12.8 \mathrm{~Hz}, 1 \mathrm{H}), 3.71-3.68(\mathrm{~m}, 1 \mathrm{H}), 3.43-3.37$ (m, 2H), $2.66(\mathrm{dd}, J=18.8,8.8 \mathrm{~Hz}, 1 \mathrm{H}), 2.60-2.49(\mathrm{~m}, 1 \mathrm{H}), 2.42-2.32(\mathrm{~m}, 1 \mathrm{H}), 2.04-1.97(\mathrm{~m}, 1 \mathrm{H}) ;{ }^{13} \mathrm{C} \mathrm{NMR}$ $\left(100 \mathrm{MHz}, \mathrm{CDCl}_{3}\right): \delta$ (ppm) 211.4, 195.5, 138.5, 134.9, 134.9, 133.0, 132.5, 129.5, 129.3, 129.2, 129.1, 124.6, 124.6, 121.8, 117.7, 85.3, 62.5, 49.4, 47.3, 46.9, 39.5, 36.4, 21.1; ESI-HRMS: calcd. for $\mathrm{C}_{29} \mathrm{H}_{23}{ }^{79} \mathrm{BrN}_{2} \mathrm{O}_{4}+\mathrm{Na}^{+}$565.0733, found 565.0734, $\mathrm{C}_{29} \mathrm{H}_{23}{ }^{81} \mathrm{BrN}_{2} \mathrm{O}_{4}+\mathrm{Na}^{+}$567.0713, found 567.0707.

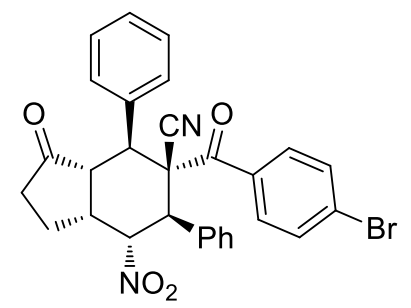

Synthesis of 12j: A solution of 2-cyclopentenone $1 \mathbf{a}(16.4 \mathrm{mg}, 0.2 \mathrm{mmol})$, (E)-2-(4-bromobenzoyl)-3-phenylacrylonitrile $\quad(31.0 \quad \mathrm{mg}, \quad 0.1 \mathrm{mmol})$, quinine C6 (6.4 mg, $0.02 \mathrm{mmol})$, thiol T1 $(1.5 \mathrm{mg}, 0.01 \mathrm{mmol})$ in $o$-xylene $(1.0 \mathrm{~mL})$ was stirred at $30{ }^{\circ} \mathrm{C}$ for $48 \mathrm{~h}$. The reaction was monitored by TLC. After completion, the intermediate was obtained by flash chromatography on silica gel (EtOAc/petroleum ether $=1 / 12-1 / 9)$. After that, the activated alkene 4a $(14.9 \mathrm{mg}, 0.1$ mmol), DIPEA $(12.9 \mathrm{mg}, 0.1 \mathrm{mmol})$ and $o$-xylene $(1.0 \mathrm{~mL})$ were added and the reaction was stirred at $50{ }^{\circ} \mathrm{C}$ for $48 \mathrm{~h}$. After completion, purification by flash chromatography on silica gel $($ EtOAc/petroleum ether $=1 / 9-1 / 7)$ gave product $\mathbf{1 2 j}: 43.9 \mathrm{mg}$, as a white solid, yield $81 \% ;[\alpha]_{\mathrm{D}}^{25}=$ $+56.0\left(c=0.2\right.$ in $\left.\mathrm{CHCl}_{3}\right)$; 91:9 er, determined by HPLC analysis [Daicel chiralcel OD-H, $n$-hexane/ $i$ - 
$\left.\operatorname{PrOH}=60 / 40,1.0 \mathrm{~mL} \mathrm{~min}^{-1}, \lambda=254 \mathrm{~nm}\right]: \mathrm{t}$ (minor) $=15.55 \mathrm{~min}, \mathrm{t}$ (major) $\left.=37.73 \mathrm{~min}\right] ;{ }^{1} \mathrm{H} \mathrm{NMR}$ (400 MHz, $\left.\mathrm{CDCl}_{3}\right): \delta(\mathrm{ppm}) 7.41-7.30(\mathrm{~m}, 10 \mathrm{H}), 7.10(\mathrm{~d}, J=8.4 \mathrm{~Hz}, 2 \mathrm{H}), 6.16(\mathrm{~d}, J=8.4 \mathrm{~Hz}, 2 \mathrm{H})$, $5.91(\mathrm{dd}, J=12.8,5.6 \mathrm{~Hz}, 1 \mathrm{H}), 4.20(\mathrm{~d}, J=12.4 \mathrm{~Hz}, 1 \mathrm{H}), 3.72-3.69(\mathrm{~m}, 1 \mathrm{H}), 3.43-3.37(\mathrm{~m}, 2 \mathrm{H})$, 2.69-2.49 (m, 2H), 2.41-2.32 (m, 1H), 2.03-1.96 (m, 1H); $\left.{ }^{13} \mathrm{C} \mathrm{NMR} \mathrm{(100} \mathrm{MHz,} \mathrm{CDCl}_{3}\right): \delta(\mathrm{ppm})$ 211.4, 195.6, 135.6, 133.0, 132.6, 131.0, 129.4, 129.1, 129.0, 128.1, 127.5, 117.9, 85.3, 62.1, 49.4, 47.3, 46.8, 39.5, 36.4, 21.1; ESI-HRMS: calcd. for $\mathrm{C}_{29} \mathrm{H}_{23}{ }^{79} \mathrm{BrN}_{2} \mathrm{O}_{4}+\mathrm{Na}^{+}$565.0733, found 565.0731, $\mathrm{C}_{29} \mathrm{H}_{23}{ }^{81} \mathrm{BrN}_{2} \mathrm{O}_{4}+\mathrm{Na}^{+}$567.0713, found 567.0717.

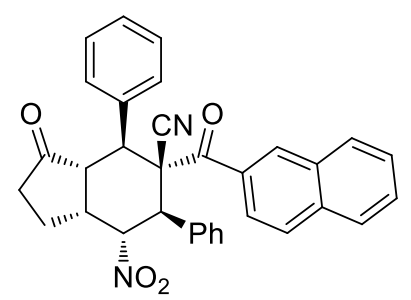

Synthesis of 12k: A solution of 2-cyclopentenone 1a (16.4 mg, $0.2 \mathrm{mmol})$, (E)-2-(2-naphthoyl)-3-phenylacrylonitrile (28.3 mg, $0.1 \mathrm{mmol})$, quinine C6 (6.4 mg, $0.02 \mathrm{mmol})$, thiol T1 $(1.5 \mathrm{mg}, 0.01 \mathrm{mmol})$ in $o$-xylene $(1.0 \mathrm{~mL})$ was stirred at $30{ }^{\circ} \mathrm{C}$ for $48 \mathrm{~h}$. The reaction was monitored by TLC. After completion, the intermediate was obtained by flash chromatography on silica gel (EtOAc/petroleum ether $=1 / 12-1 / 9)$. After that, the activated alkene $4 \mathbf{a}(14.9 \mathrm{mg}, 0.1 \mathrm{mmol})$, DIPEA (12.9 mg, 0.1 mmol) and $o$-xylene $(1.0 \mathrm{~mL})$ were added and the reaction was stirred at $50{ }^{\circ} \mathrm{C}$ for $48 \mathrm{~h}$. After completion, purification by flash chromatography on silica gel (EtOAc/petroleum ether $=1 / 9-1 / 7)$ gave product 12k: $41.6 \mathrm{mg}$, as a white solid, yield $81 \%$; $[\alpha]_{\mathrm{D}}{ }^{25}=+64.0\left(c=0.5\right.$ in $\left.\mathrm{CHCl}_{3}\right) ; 90: 10 \mathrm{er}$, determined by HPLC analysis [Daicel chiralpak AD-H, $n$-hexane $/ i-\mathrm{PrOH}=60 / 40,1.0 \mathrm{~mL} \mathrm{~min}{ }^{-1}, \lambda=$ $254 \mathrm{~nm}]: \mathrm{t}($ minor $)=12.31 \mathrm{~min}, \mathrm{t}($ major $)=21.15 \mathrm{~min}] ;{ }^{1} \mathrm{H} \mathrm{NMR}\left(400 \mathrm{MHz}, \mathrm{CDCl}_{3}\right): \delta(\mathrm{ppm})$ 7.63-7.61 (m, 2H), 7.47-7.36 (m, 7H), 7.34-7.25 (m, 5H), $6.72(\mathrm{~s}, 1 \mathrm{H}), 6.46(\mathrm{dd}, J=8.4,1.6 \mathrm{~Hz}$, 1H), $5.95(\mathrm{dd}, J=12.4,5.6 \mathrm{~Hz}, 1 \mathrm{H}), 4.51(\mathrm{~d}, J=12.8 \mathrm{~Hz}, 1 \mathrm{H}), 3.78$ (d, $J=12.8 \mathrm{~Hz}, 1 \mathrm{H}), 3.49-3.39$ $(\mathrm{m}, 2 \mathrm{H}), 2.72-2.54(\mathrm{~m}, 2 \mathrm{H}), 2.43-2.33(\mathrm{~m}, 1 \mathrm{H}), 2.05-1.98(\mathrm{~m}, 1 \mathrm{H}) ;{ }^{13} \mathrm{C} \mathrm{NMR}\left(100 \mathrm{MHz}, \mathrm{CDCl}_{3}\right): \delta$ (ppm) 211.6, 196.2, 134.5, 134.3, 133.3, 132.9, 131.2, 129.4, 129.3, 129.0, 128.5, 128.4, 127.6, 127.4, 126.7, 122.5, 118.1, 85.4, 62.3, 49.5, 47.4, 46.9, 39.6, 36.4, 21.1; ESI-HRMS: calcd. for $\mathrm{C}_{33} \mathrm{H}_{26} \mathrm{~N}_{2} \mathrm{O}_{4}+\mathrm{Na}^{+}$537.1785, found 537.1786.

\section{Reaction at a $1.0 \mathrm{mmol}$ scale}

A solution of 2-cyclopentenone 1a (164 mg, $2.0 \mathrm{mmol})$, (E)-2-benzoyl-3-(4-bromophenyl) acrylonitrile (310 $\mathrm{mg}, 1.0 \mathrm{mmol})$, quinine C6 (64 mg, $0.2 \mathrm{mmol})$, thiol T1 (15 $\mathrm{mg}, 0.1 \mathrm{mmol})$ in $o$ xylene $(10.0 \mathrm{~mL})$ was stirred at $30^{\circ} \mathrm{C}$ for $72 \mathrm{~h}$. The reaction was monitored by TLC. After completion, the intermediate was obtained by flash chromatography on silica gel (EtOAc/petroleum ether $=1 / 10)$. 
Then the activated alkene 4a (149 mg, $1.0 \mathrm{mmol}$ ), $N, N$-diisopropylenthylamine (DIPEA, $129 \mathrm{mg}, 1.0$ $\mathrm{mmol}$ ) and $o$-xylene $\left(10.0 \mathrm{~mL}\right.$ ) were added and the reaction was stirred at $50{ }^{\circ} \mathrm{C}$ for $48 \mathrm{~h}$ (monitored by TLC). After completion, the product $\mathbf{1 2 f}$ was obtained by flash chromatography on silica gel $($ EtOAc/petroleum ether $=1 / 8): 406 \mathrm{mg}$, as a white solid, yield 75\%, 94.5:5.5 er.

\section{Procedure for the cross $\mathrm{RC}$ reaction-initiated $[4+2]$ annulation}

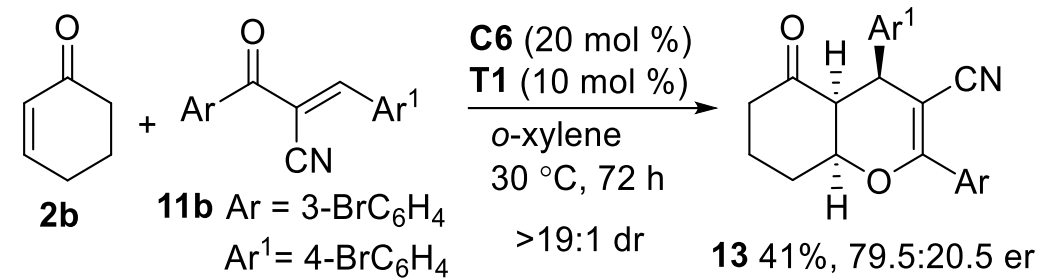

A solution of 2-cyclohexenone $\mathbf{2 b}$ (1.0 mmol, 10.0 equiv), $\alpha$-cyanochalcone $\mathbf{1 1 b}(0.1 \mathrm{mmol}, 1.0$ equiv), quinine C6 (20 mol \%), thiol T1 (10 mol \%) in $o$-xylene $(0.4 \mathrm{~mL})$ was stirred at $30{ }^{\circ} \mathrm{C}$ for 72 h. The reaction was monitored by TLC. The product $\mathbf{1 3}$ was obtained by flash chromatography on silica gel (EtOAc/petroleum ether): $20.8 \mathrm{mg}$, as a white solid, yield $41 \%$; $[\alpha]_{\mathrm{D}}{ }^{25}=+84.0(c=0.3$ in $\mathrm{CHCl}_{3}$ ); 79.5:20.5 er, determined by HPLC analysis [Daicel chiralpak AD-H, $n$-hexane/ $i$-PrOH = 60/40, $\left.1.0 \mathrm{~mL} \mathrm{~min}^{-1}, \lambda=254 \mathrm{~nm}\right]: \mathrm{t}$ (minor) $=8.85 \mathrm{~min}, \mathrm{t}$ (major) $\left.=20.00 \mathrm{~min}\right] ;{ }^{1} \mathrm{H} \mathrm{NMR}(400 \mathrm{MHz}$, $\left.\mathrm{CDCl}_{3}\right): \delta(\mathrm{ppm}) 7.84-7.83(\mathrm{~m}, 1 \mathrm{H}), 7.81-7.78(\mathrm{~m}, 1 \mathrm{H}), 7.62-7.59(\mathrm{~m}, 1 \mathrm{H}), 7.53-7.50(\mathrm{~m}, 2 \mathrm{H}), 7.32$ $(\mathrm{t}, J=8.0 \mathrm{~Hz}, 1 \mathrm{H}), 7.15-7.12(\mathrm{~m}, 2 \mathrm{H}), 4.59(\mathrm{~d}, J=2.8 \mathrm{~Hz}, 1 \mathrm{H}), 4.48(\mathrm{~s}, 1 \mathrm{H}), 2.73(\mathrm{~d}, J=3.2 \mathrm{~Hz}$, 1H), 2.56-2.52 (m, 1H), 2.44-2.36 (m, 2H), 2.07-2.03 (m, 2H), 1.95-1.86 (m, 1H); ${ }^{13} \mathrm{C}$ NMR (100 $\left.\mathrm{MHz}, \mathrm{CDCl}_{3}\right): \delta$ (ppm) 205.2, 164.0, 140.4, 134.3, 134.0, 132.3, 130.9, 129.9, 129.7, 127.0, 122.4, 121.8, 118.9, 85.0, 74.4, 54.3, 40.7, 37.6, 29.2, 21.6; ESI-HRMS: calcd. for $\mathrm{C}_{22} \mathrm{H}_{17}{ }^{79} \mathrm{BrNO}_{2}+\mathrm{Na}^{+}$ 507.9518, found 507.9516. $\mathrm{C}_{22} \mathrm{H}_{17}{ }^{81} \mathrm{BrNO}_{2}+\mathrm{Na}^{+} 509.9498$, found 509.9503.

\section{More attempts for the cross $\mathrm{RC}$ reaction-initiated $[4+2]$ annulations}

The $[4+2]$ annulation reactions between 2-cyclohexenone $\mathbf{2 b}$ and $\alpha$-cyano chalcone derivatives $\mathbf{1 1}$ generally proceeded not well, and excess $\mathbf{2 b}$ (10.0 equiv) was required for better conversions. For simple $\alpha$-cyano chalcone 11a, the desired product could not be isolated due to the low yield and some inseparable impurities. Similar phenomena were observed for some other $\alpha$-cyano chalcone derivatives 11 with electron-withdrawing groups on the phenyl ring. We have made efforts to optimize the reaction conditions by employing 11a as the substrate, and a better yield with a low er value was obtained under the catalysis of $\mathbf{C 2 7}$ and T18. In addition, cyclohept-2-enone 1c and $\alpha^{\prime}$-benzylidene 2-cyclopentenone 6 were also applied to the $[4+2]$ annulation, but the reaction were not satisfactory. 


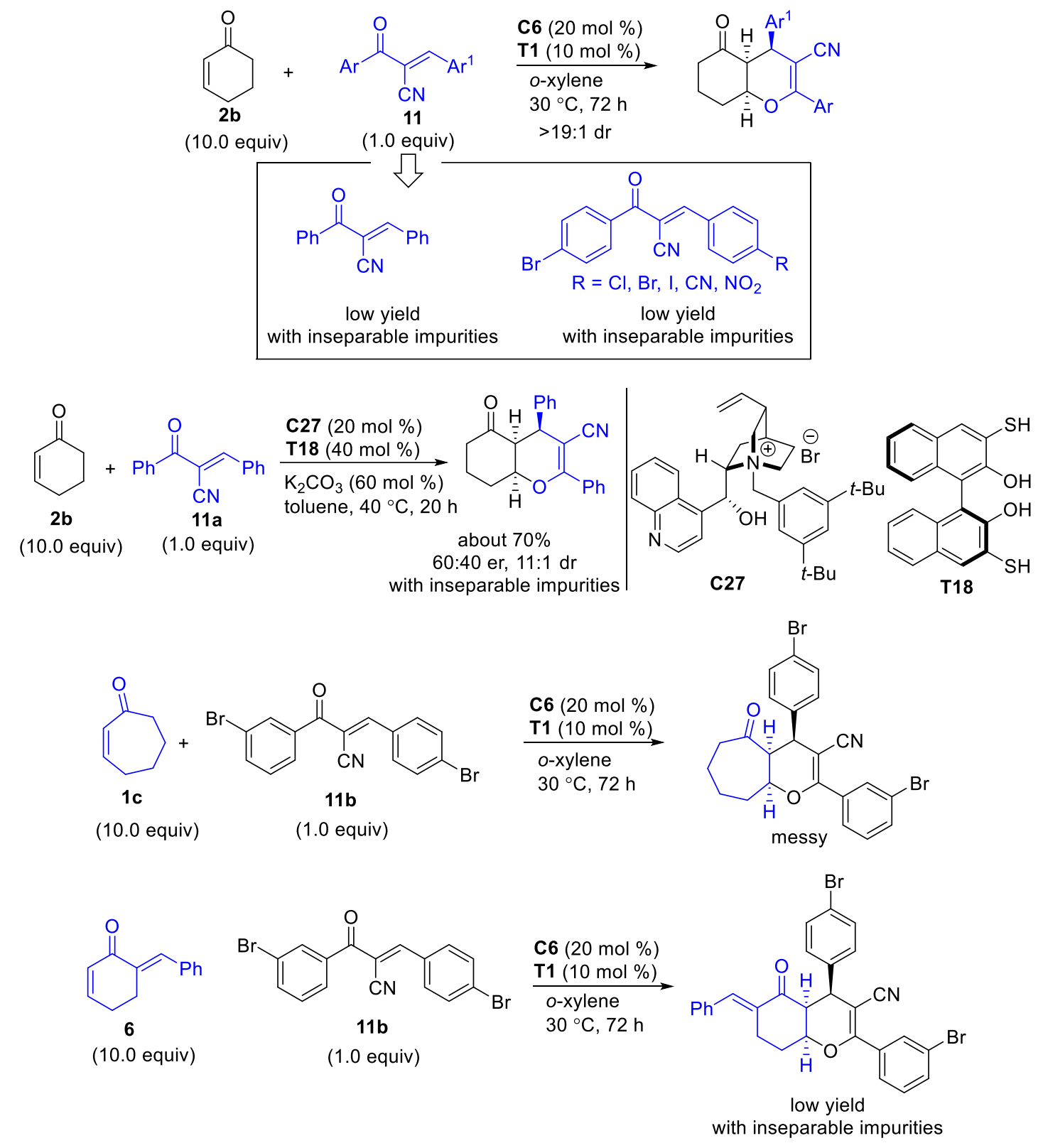

\section{Synthetic transfomations of the annulation products 5a and 12a}<smiles></smiles>

5a $93: 7$ er

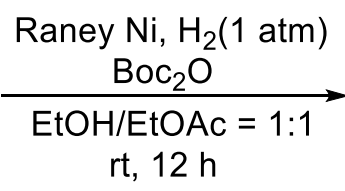

$\mathrm{rt}, 12 \mathrm{~h}$<smiles>CN1C(=O)[C@]23C(=O)CC[C@H]2C(N)C(c2ccccc2)[C@]3(C(C)(C)C)c2ccccc21</smiles>

$1480 \%, 93: 7$ er

A solution of $\mathbf{5 a}(0.1 \mathrm{mmol}, 44.0 \mathrm{mg}, 93: 7 \mathrm{er}), \mathrm{Boc}_{2} \mathrm{O}(0.15 \mathrm{mmol}, 32.7 \mathrm{mg})$ in the solvent of EtOH $(0.5 \mathrm{~mL})$ and EtOAc $(0.5 \mathrm{~mL})$ was combined with Raney Ni (W2; $50.0 \mathrm{mg}$, wet weight). The mixture was stirred at room temperature under hydrogen $(1 \mathrm{~atm})$ for $12 \mathrm{~h}$. The mixture was filtered through a bed of celite and concentrated under reduced pressure. The residue was purified by flash 
chromatography $($ EtOAc/petroleum ether $=1: 5)$ to gived compound 14: $40.8 \mathrm{mg}$, as a white solid, yield 80\%; $[\alpha]_{\mathrm{D}}{ }^{25}=+66.2\left(c=0.65\right.$ in $\left.\mathrm{CHCl}_{3}\right)$; 93:7 er, determined by HPLC analysis [Daicel chiralpak OD-H, $n$-hexane/ $i$-PrOH $\left.=60 / 40,1.0 \mathrm{~mL} \mathrm{~min}^{-1}, \lambda=254 \mathrm{~nm}\right]: \mathrm{t}$ (minor) $=8.04 \mathrm{~min}, \mathrm{t}$ (major) $=9.17 \mathrm{~min}] ;{ }^{1} \mathrm{H} \mathrm{NMR}\left(400 \mathrm{MHz}, \mathrm{CDCl}_{3}\right): \delta(\mathrm{ppm}) 7.72(\mathrm{dd}, J=7.8 \mathrm{~Hz}, 1.1 \mathrm{~Hz}, 1 \mathrm{H}), 7.56-7.50(\mathrm{~m}$, 2H), 7.49-7.35 (m, 4H), 7.28-7.22 (m, 1H), 6.91 (d, J = 7.8 Hz, 1H), 5.14-5.09 (m, 1H), 4.74 (brs, $1 \mathrm{H}), 4.66(\mathrm{~d}, J=12.2 \mathrm{~Hz}, 1 \mathrm{H}), 3.40-3.26(\mathrm{~m}, 1 \mathrm{H}), 3.22$ (d, $J=9.3 \mathrm{~Hz}, 1 \mathrm{H}), 3.18(\mathrm{~s}, 3 \mathrm{H}), 2.67-2.56$ (m, 1H), 2.45-2.38 (m, 1H), 2.31-2.21 (m, 1H), 2.11-2.04 (m, 1H), 1.31 (s, 9H); ${ }^{13} \mathrm{C}$ NMR (100 $\left.\mathrm{MHz}, \mathrm{CDCl}_{3}\right): \delta$ (ppm) 214.8, 173.1, 155.1, 143.3, 133.2, 130.9, 129.7, 129.4, 129.0, 126.2, 124.7, 124.2, 112.1, 111.5, 109.2, 80.1, 53.3, 49.3, 49.1, 48.3, 42.8, 39.4, 38.5, 28.2, 26.9, 22.4; ESI-HRMS: calcd. for $\mathrm{C}_{30} \mathrm{H}_{30} \mathrm{~N}_{4} \mathrm{O}_{4}+\mathrm{Na}^{+}$533.2159, found 533.2158.

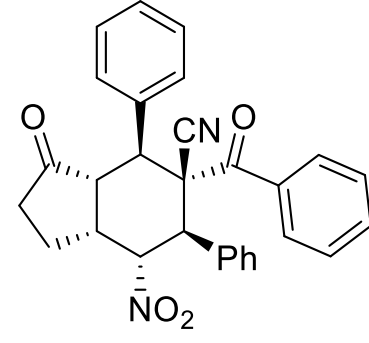

12a $91.5: 8.5$ er

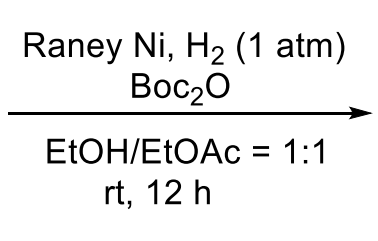

$\mathrm{rt}, 12 \mathrm{~h}$

A solution of 12a $(0.1 \mathrm{mmol}, 46.4 \mathrm{mg}, 91.5: 8.5 \mathrm{er})$, $\mathrm{Boc}_{2} \mathrm{O}(0.15 \mathrm{mmol}, 32.7 \mathrm{mg})$ in the solvent of EtOH (0.5 mL) and EtOAc (0.5 mL) was combined with Raney Ni (W2; $50.0 \mathrm{mg}$, wet weight). The mixture was stirred at room temperature under hydrogen $(1 \mathrm{~atm})$ for $12 \mathrm{~h}$. The mixture was filtered through a bed of celite and condensed under reduced pressure. The residue was purified by flash chromatography (EtOAc/petroleum ether = 1:5) to gived compound 15: $46.4 \mathrm{mg}$, as a white solid, yield 87\%; $[\alpha]_{\mathrm{D}}{ }^{25}=+37.1\left(c=0.75\right.$ in $\left.\mathrm{CHCl}_{3}\right)$; 91.5:8.5 er, determined by HPLC analysis [Daicel chiralpak AD-H, $n$-hexane $\left./ i-\mathrm{PrOH}=80 / 20,1.0 \mathrm{~mL} \mathrm{~min}^{-1}, \lambda=254 \mathrm{~nm}\right]: \mathrm{t}$ (minor) $=8.95 \mathrm{~min}, \mathrm{t}$ (major) $=10.13 \mathrm{~min}] ;{ }^{1} \mathrm{H}$ NMR $\left(400 \mathrm{MHz}, \mathrm{CDCl}_{3}\right): \delta(\mathrm{ppm}) 7.47-7.20(\mathrm{~m}, 10 \mathrm{H}), 7.16(\mathrm{t}, J=7.5 \mathrm{~Hz}, 1 \mathrm{H})$, $6.92(\mathrm{t}, J=7.9 \mathrm{~Hz}, 2 \mathrm{H}), 6.31-6.22(\mathrm{~m}, 2 \mathrm{H}), 5.04(\mathrm{brs}, 1 \mathrm{H}), 4.32$ (d, $J=8.5 \mathrm{~Hz}, 1 \mathrm{H}), 3.69$ (d, $J=12.7$ $\mathrm{Hz}, 1 \mathrm{H}), 3.62(\mathrm{~d}, J=13.3 \mathrm{~Hz}, 1 \mathrm{H}), 3.31(\mathrm{dd}, J=13.3,6.5 \mathrm{~Hz}, 1 \mathrm{H}), 3.17-3.14(\mathrm{~m}, 1 \mathrm{H}), 2.63-2.51(\mathrm{~m}$, 1H), 2.41-2.13 (m, 3H), 1.29 (s, 9H); $\left.{ }^{13} \mathrm{C} \mathrm{NMR} \mathrm{(100} \mathrm{MHz,} \mathrm{CDCl}_{3}\right): \delta$ (ppm) 213.9, 198.0, 155.0, $137.5,134.4,134.3,131.8,129.9,128.9,128.8,128.6,127.4,126.6,118.3,80.0,63.6,50.5,49.8$, 46.9, 40.3, 36.6, 28.2, 20.3; ESI-HRMS: calcd. for $\mathrm{C}_{34} \mathrm{H}_{34} \mathrm{~N}_{2} \mathrm{O}_{4}+\mathrm{Na}^{+}$557.2411, found 557.2409. 


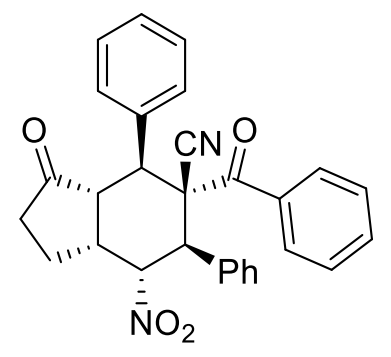

12a $91.5: 8.5$ er

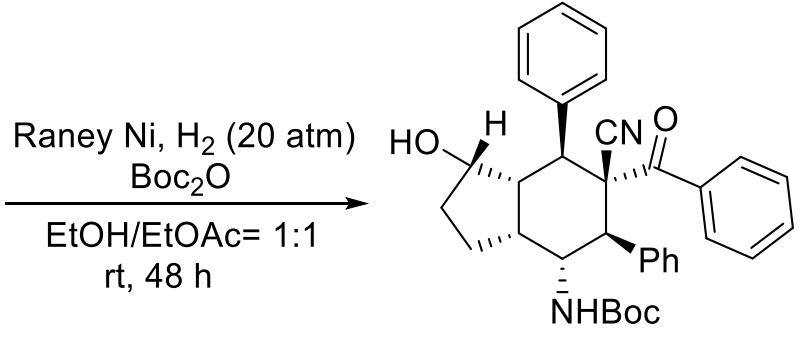

$1682 \%, 91.5: 8.5$ er

A solution of 12a $(0.1 \mathrm{mmol}, 46.4 \mathrm{mg}, 91.5: 8.5 \mathrm{er})$, $\mathrm{Boc}_{2} \mathrm{O}(0.15 \mathrm{mmol}, 32.7 \mathrm{mg})$ in the solvent of $\mathrm{EtOH}(0.5 \mathrm{~mL})$ and EtOAc $(0.5 \mathrm{~mL})$ was combined with Raney Ni (W2; $50.0 \mathrm{mg}$, wet weight). The mixture was stirred at room temperature under hydrogen (20 atm) for $48 \mathrm{~h}$. The mixture was filtered through a bed of celite and condensed under reduced pressure. The residue was purified by flash chromatography $($ EtOAc/petroleum ether $=1: 4)$ to gived compound 16: $44.0 \mathrm{mg}$, as a white solid, yield $82 \% ;[\alpha]_{\mathrm{D}}{ }^{25}=-12.6\left(c=0.35\right.$ in $\left.\mathrm{CHCl}_{3}\right)$; 91.5:8.5 er, $>19: 1 \mathrm{dr}$, determined by HPLC analysis

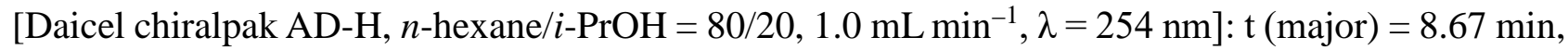
$\mathrm{t}($ minor $)=12.18 \mathrm{~min}] ;{ }^{1} \mathrm{H} \mathrm{NMR}\left(400 \mathrm{MHz}, \mathrm{CDCl}_{3}\right): \delta(\mathrm{ppm}) 7.51-7.00(\mathrm{~m}, 11 \mathrm{H}), 6.85(\mathrm{t}, J=7.7$ $\mathrm{Hz}, 2 \mathrm{H}), 6.18(\mathrm{~d}, J=7.8 \mathrm{~Hz}, 2 \mathrm{H}), 4.81$ (brs, 1H), 4.37-4.33 (m, 2H), 3.69-3.60 (m, 2H), 3.17-3.02 (m, 1H), 2.96-2.82 (m, 1H), 2.10-2.07 (m, 1H), 2.00-1.85 (m, 1H), 1.81-1.72 (m, 2H), 1.22 (s, 9H), 0.67 (brs, 1H); ${ }^{13} \mathrm{C}$ NMR (100 MHz, $\mathrm{CDCl}_{3}$ ): $\delta$ (ppm) 198.9, 155.1, 138.2, 137.9, 135.1, 130.0, 129.1, $128.8,128.3,128.1,127.3,126.6,119.0$, 79.5, 76.0, 64.4, 50.9, 49.7, 47.1, 44.5, 40.0, 28.2, 20.9; ESIHRMS: calcd. for $\mathrm{C}_{34} \mathrm{H}_{37} \mathrm{~N}_{2} \mathrm{O}_{4}+\mathrm{H}^{+} 537.2748$, found 537.2750.<smiles>N#C[C@@]1(c2ccccc2)[C@H](c2ccccc2)[C@@H]2C(=O)CC[C@@H]2[C@H]([N+](=O)[O-])[C@H]1c1ccccc1</smiles>

12a $91.5: 8.5 \mathrm{er}$

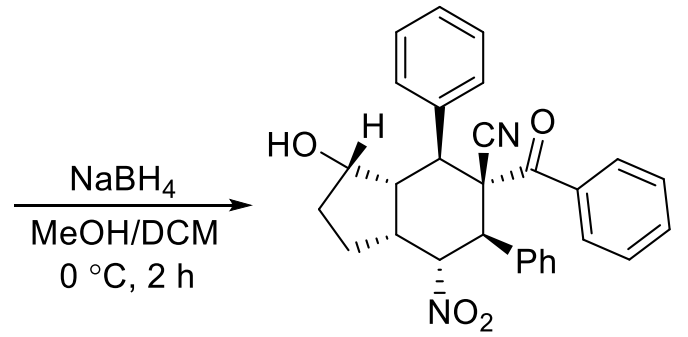

$1777 \%, 91.5: 8.5 \mathrm{er},>19: 1 \mathrm{dr}$

To a solution of 12a $(0.1 \mathrm{mmol}, 46.4 \mathrm{mg}, 91.5: 8.5 \mathrm{er})$ in the solvent of $\mathrm{MeOH}(0.5 \mathrm{~mL})$ and DCM $(0.5 \mathrm{~mL})$ was added $\mathrm{NaBH}_{4}(0.15 \mathrm{mmol}, 5.7 \mathrm{mg})$ at $0{ }^{\circ} \mathrm{C}$. After $2 \mathrm{~h}$, the mixture was quenched with saturated $\mathrm{NH}_{4} \mathrm{Cl}$ solution. The phases were separated and the aqueous phase was extracted with DCM $(2 \times 5 \mathrm{~mL})$. The combined organic phases were washed with brine $(10 \mathrm{~mL})$ before being dried $\left(\mathrm{Na}_{2} \mathrm{SO}_{4}\right)$ and concentrated in vacuum. The residue was purified by flash chromatography $($ EtOAc/petroleum ether $=1: 6)$ to gived compound 17: $35.9 \mathrm{mg}$, as a white solid, yield $77 \% ;[\alpha]_{\mathrm{D}}^{25}=$ -26.7 ( $c=0.15$ in $\left.\mathrm{CHCl}_{3}\right)$; 91.5:8.5 er, >19:1 dr, determined by HPLC analysis [Daicel chiralpak AD- 


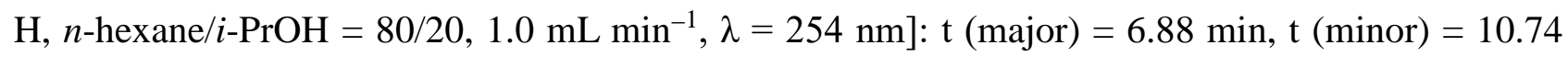
min]; ${ }^{1} \mathrm{H}$ NMR (400 MHz, $\left.\mathrm{CDCl}_{3}\right): \delta(\mathrm{ppm}) 7.38-7.14(\mathrm{~m}, 10 \mathrm{H}), 7.13-7.08(\mathrm{~m}, 1 \mathrm{H}), 6.86$ (t, $J=7.9$ $\mathrm{Hz}, 2 \mathrm{H}), 6.19(\mathrm{dd}, J=8.2,1.4 \mathrm{~Hz}, 2 \mathrm{H}), 5.72(\mathrm{dd}, J=12.6,5.8 \mathrm{~Hz}, 1 \mathrm{H}), 4.48-4.42(\mathrm{~m}, 1 \mathrm{H}), 4.31(\mathrm{~d}$, $J=12.6 \mathrm{~Hz}, 1 \mathrm{H}), 3.79(\mathrm{~d}, J=12.6 \mathrm{~Hz}, 1 \mathrm{H}), 3.30-3.23(\mathrm{~m}, 1 \mathrm{H}), 3.10-3.02(\mathrm{~m}, 1 \mathrm{H}), 2.33-2.22(\mathrm{~m}$, 1H), 2.19-2.10 (m, 1H), 1.88-1.79 (m, 1H), 1.60-1.51 (m, 1H), 0.64 (brs, 1H); ${ }^{13} \mathrm{C} \mathrm{NMR} \mathrm{(100} \mathrm{MHz,}$ $\left.\mathrm{CDCl}_{3}\right): \delta(\mathrm{ppm}) 197.6,137.5,137.6,133.5,132.3,129.7,128.8,127.8,127.0,119.0,86.4,76.1,63.4$, 47.4, 47.4, 44.8, 40.8, 30.6, 21.6; ESI-HRMS: calcd. for $\mathrm{C}_{29} \mathrm{H}_{26} \mathrm{~N}_{2} \mathrm{O}_{4}+\mathrm{Na}^{+} 489.1785$, found 489.1786.
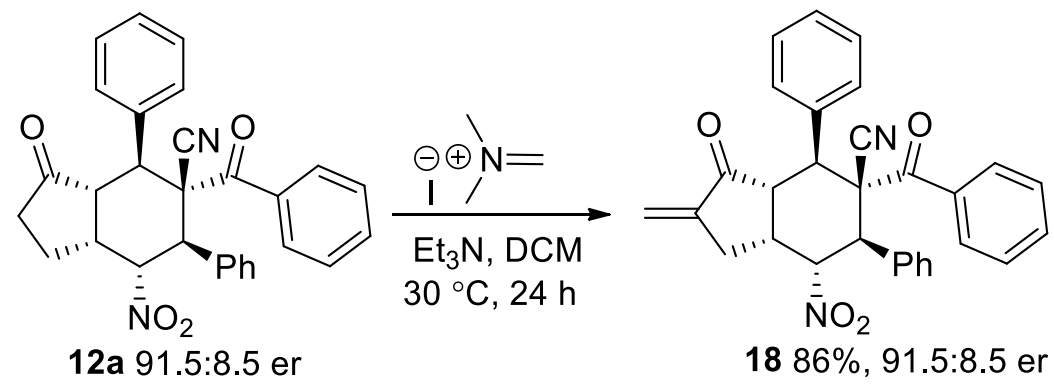

To a solution of $\mathbf{1 2 a}(0.1 \mathrm{mmol}, 46.4 \mathrm{mg}, 91.5: 8.5 \mathrm{er})$ in dry DCM $(1.0 \mathrm{~mL})$ was added $\mathrm{Et}_{3} \mathrm{~N}(0.2$ $\mathrm{mmol}, 20.2 \mathrm{mg})$ at room temperature. The mixture was stirred for $15 \mathrm{~min}$ and Eschensomer's salt (0.2 mmol, $37 \mathrm{mg}$ ) was added, and then continuously stirred for $24 \mathrm{~h}$ at $30{ }^{\circ} \mathrm{C}$. The mixture was quenched with saturated $\mathrm{NH}_{4} \mathrm{Cl}$ solution. The phases were separated and the aqueous phase was extracted with DCM $(2 \times 5 \mathrm{~mL})$. The combined organic phases were washed with brine $(10 \mathrm{~mL})$ before being dried $\left(\mathrm{Na}_{2} \mathrm{SO}_{4}\right)$ and concentrated in vacuum. The residue was purified by flash chromatography $($ EtOAc/petroleum ether $=1: 12)$ to gived compound 18: $41.1 \mathrm{mg}$, as a white solid, yield $86 \% ;[\alpha]_{\mathrm{D}}{ }^{25}$ $=+46.8(c=0.8$ in $\mathrm{MeOH})$; 91.5:8.5 er, determined by HPLC analysis [Daicel chiralpak IE, $n$ hexane $\left./ i-\mathrm{PrOH}=80 / 20,1.0 \mathrm{~mL} \mathrm{~min}^{-1}, \lambda=254 \mathrm{~nm}\right]: \mathrm{t}($ minor $)=12.32 \mathrm{~min}, \mathrm{t}$ (major) $\left.=14.68 \mathrm{~min}\right]$; ${ }^{1} \mathrm{H}$ NMR (400 MHz, CD $\left.3 \mathrm{OD}\right): \delta(\mathrm{ppm})$ 7.47-7.46 (m, 3H), 7.35-7.20 (m, 8H), $6.94(\mathrm{t}, J=8.0 \mathrm{~Hz}$, 2H), $6.30(\mathrm{~d}, J=7.6 \mathrm{~Hz}, 2 \mathrm{H}), 6.09-6.04(\mathrm{~m}, 2 \mathrm{H}), 5.61(\mathrm{~s}, 1 \mathrm{H}), 3.80$ (d, $J=13.2 \mathrm{~Hz}, 1 \mathrm{H}), 3.61(\mathrm{dd}, J$ $=13.2,6.4 \mathrm{~Hz}, 1 \mathrm{H}), 3.49-3.45(\mathrm{~m}, 1 \mathrm{H}), 3.40-3.32(\mathrm{~m}, 1 \mathrm{H}), 2.62(\mathrm{dd}, J=16.4,6.8 \mathrm{~Hz}, 1 \mathrm{H}) ;{ }^{13} \mathrm{C} \mathrm{NMR}$ (100 MHz, CD 3 OD): $\delta$ (ppm) 200.8, 196.2, 142.0, 137.3, 134.0, 133.4, 131.7, 128.8, 128.7, 128.4, 128.3, 127.1, 126.5, 120.3, 117.8, 85.2, 62.1, 53.4, 49.7, 36.8, 27.5; ESI-HRMS: calcd. for $\mathrm{C}_{30} \mathrm{H}_{24} \mathrm{~N}_{2} \mathrm{O}_{4}+\mathrm{Na}^{+} 499.1628$, found 499.1624 . 


\section{Crystal data and structure refinement for enantiopure $5 \mathrm{~g}$ and $12 \mathrm{f}$}
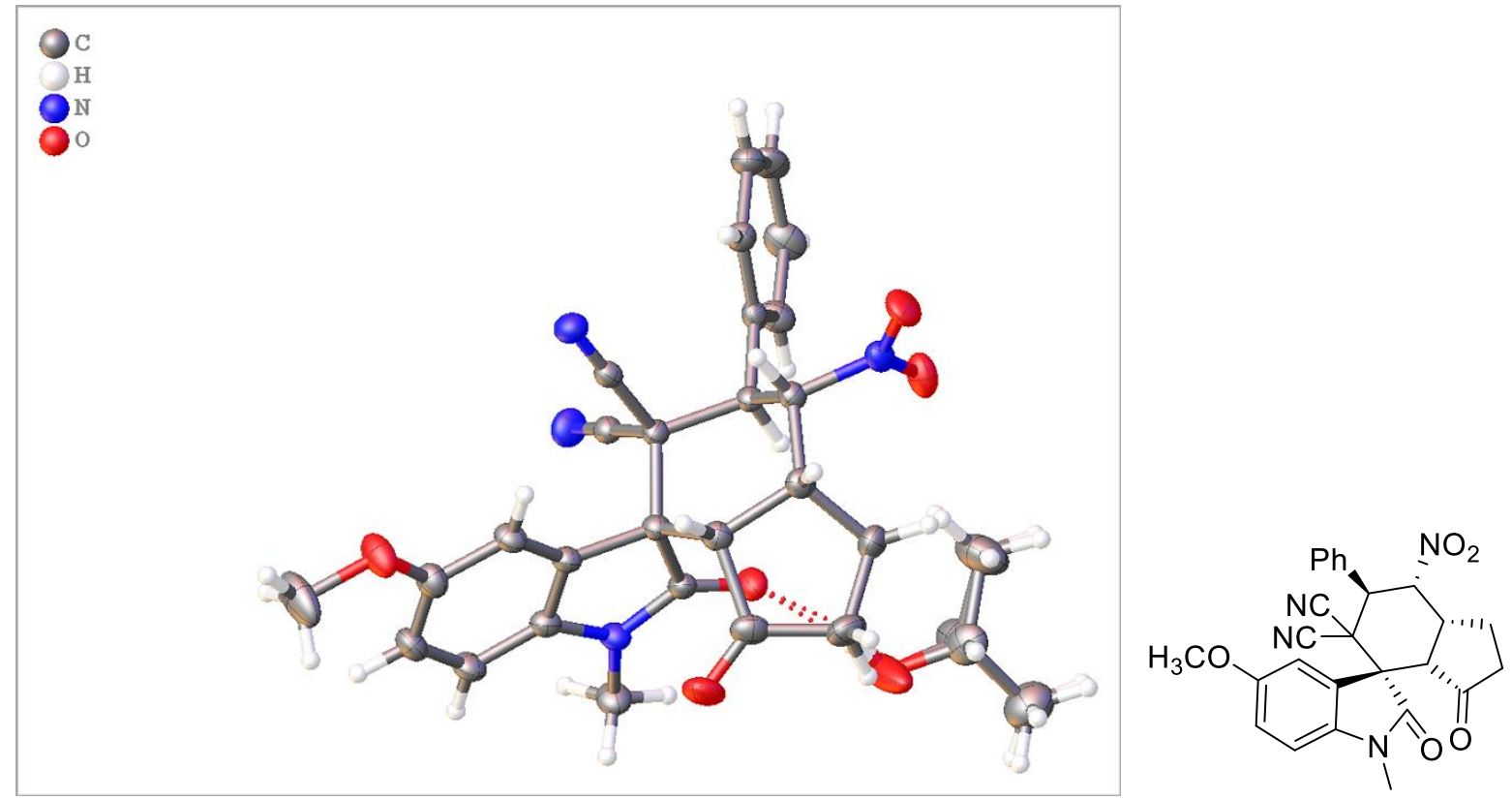

$5 g$

Identification code

Empirical formula

Formula weight

Temperature/K

Crystal system

Space group

$\mathrm{a} / \AA$

$\mathrm{b} / \AA$

$\mathrm{c} / \AA$

$\alpha /{ }^{\circ}$

$\beta /{ }^{\circ}$

$\gamma /{ }^{\circ}$

Volume $/ \AA^{3}$

$\mathrm{Z}$

$\rho_{\text {calc }} \mathrm{g} / \mathrm{cm}^{3}$

$\mu / \mathrm{mm}^{-1}$

$\mathrm{F}(000)$

Crystal size $/ \mathrm{mm}^{3}$

Radiation

$2 \Theta$ range for data collection/ ${ }^{\circ}$

Index ranges

Reflections collected

Independent reflections

Data/restraints/parameters 5g (CCDC 1877057)

$\mathrm{C}_{29} \mathrm{H}_{30} \mathrm{~N}_{4} \mathrm{O}_{6}$

530.57

136

orthorhombic

$\mathrm{P} 22_{1} 2_{1}$

9.25485(18)

9.9771(2)

29.5378(5)

90

90

90

2727.40(9)

4

1.292

0.754

1120.0

$0.5 \times 0.4 \times 0.1$

$\mathrm{CuK} \alpha(\lambda=1.54184)$

9.356 to 134.152

$-10 \leq \mathrm{h} \leq 11,-11 \leq \mathrm{k} \leq 11,-25 \leq 1 \leq 35$

14563

$4852\left[R_{\text {int }}=0.0372, R_{\text {sigma }}=0.0337\right]$

$4852 / 0 / 364$ 
Goodness-of-fit on $\mathrm{F}^{2}$

Final $\mathrm{R}$ indexes $[\mathrm{I}>=2 \sigma(\mathrm{I})]$

Final $\mathrm{R}$ indexes [all data]

Largest diff. peak/hole / e $\AA^{-3}$

Flack parameter
1.034

$\mathrm{R}_{1}=0.0444, \mathrm{wR}_{2}=0.1159$

$\mathrm{R}_{1}=0.0471, \mathrm{wR}_{2}=0.1190$

$0.22 /-0.25$

$0.09(10)$

\section{oc \\ H \\ (2)}
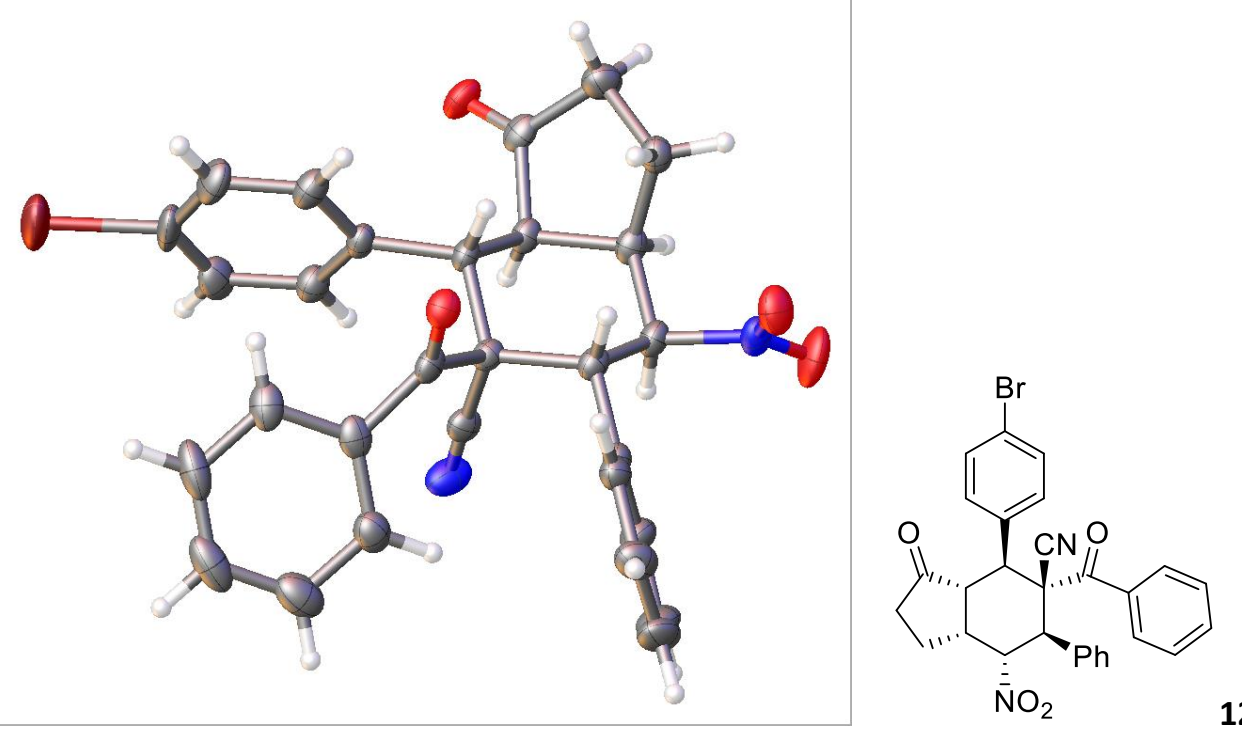

Identification code

Empirical formula

Formula weight

Temperature/K

Crystal system

Space group

$\mathrm{a} / \AA ̊$

$\mathrm{b} / \AA$

$\mathrm{c} / \AA ̊ \AA$

$\alpha /{ }^{\circ}$

$\beta /{ }^{\circ}$

$\gamma /{ }^{\circ}$

Volume/ $/ \AA^{3}$

Z

pcalcg $/ \mathrm{cm}^{3}$

$\mu / \mathrm{mm}^{-1}$

$\mathrm{F}(000)$

Crystal size $/ \mathrm{mm}^{3}$

Radiation

$2 \Theta$ range for data collection/ ${ }^{\circ}$

Index ranges

Reflections collected

Independent reflections 12f (CCDC 1877058)

$\mathrm{C}_{29} \mathrm{H}_{23} \mathrm{BrN}_{2} \mathrm{O}_{4}$

543.40

142

orthorhombic

P212121

7.6578(2)

11.9171(4)

27.6310(7)

90

90

90

2521.56(13)

4

1.431

2.536

1112.0

$0.65 \times 0.4 \times 0.3$

$\mathrm{CuK} \alpha(\lambda=1.54184)$

9.802 to 145.462

$-5 \leq \mathrm{h} \leq 9,-13 \leq \mathrm{k} \leq 14,-34 \leq 1 \leq 22$

11266

$4854\left[R_{\text {int }}=0.0326, R_{\text {sigma }}=0.0371\right]$ 
Data/restraints/parameters

Goodness-of-fit on $\mathrm{F}^{2}$

Final $\mathrm{R}$ indexes $[\mathrm{I}>=2 \sigma(\mathrm{I})]$

Final $\mathrm{R}$ indexes [all data]

Largest diff. peak/hole / e $\AA^{-3}$

Flack parameter
$4854 / 0 / 325$

1.026

$\mathrm{R}_{1}=0.0550, \mathrm{wR}_{2}=0.1472$

$\mathrm{R}_{1}=0.0569, \mathrm{wR}_{2}=0.1501$

1.23/-0.96

$-0.005(12)$ 


\section{DFT calculations of the key intermediates for the asymmetric cross}

\section{Rauhut-Currier reaction and proposed catalytic mechanism}

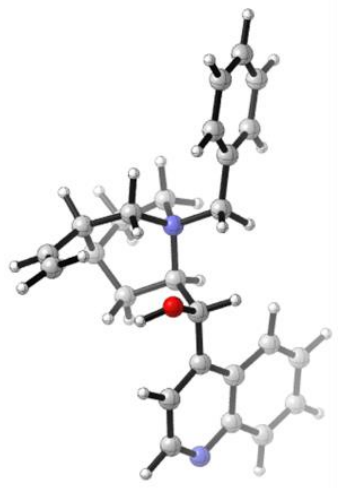

C2-A

$0.0 \mathrm{kcal} / \mathrm{mol}$

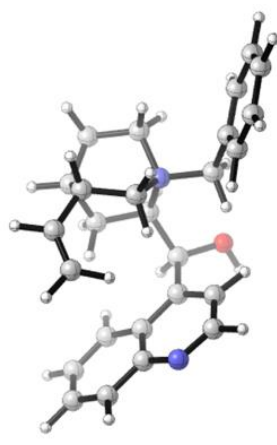

C2-B

$3.1 \mathrm{kcal} / \mathrm{mol}$

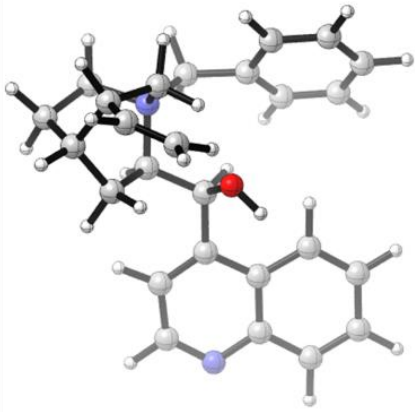

$\mathrm{C} 2-\mathrm{C}$

$9.0 \mathrm{kcal} / \mathrm{mol}$

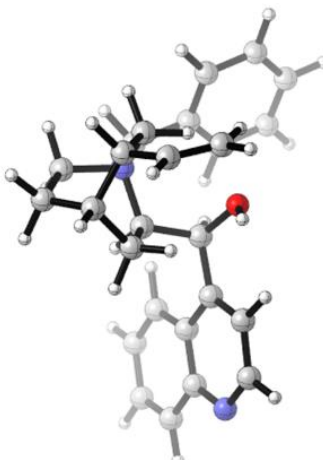

C2-D

$6.6 \mathrm{kcal} / \mathrm{mol}$

Figure S1. The conformations and energies of PTC C2.

The conformations and energies of chiral PTC C2 were calculated as outlined Figure S1. The structure as C2-A with the lowest energy was selected as the most favorable conformation for the following calculations.
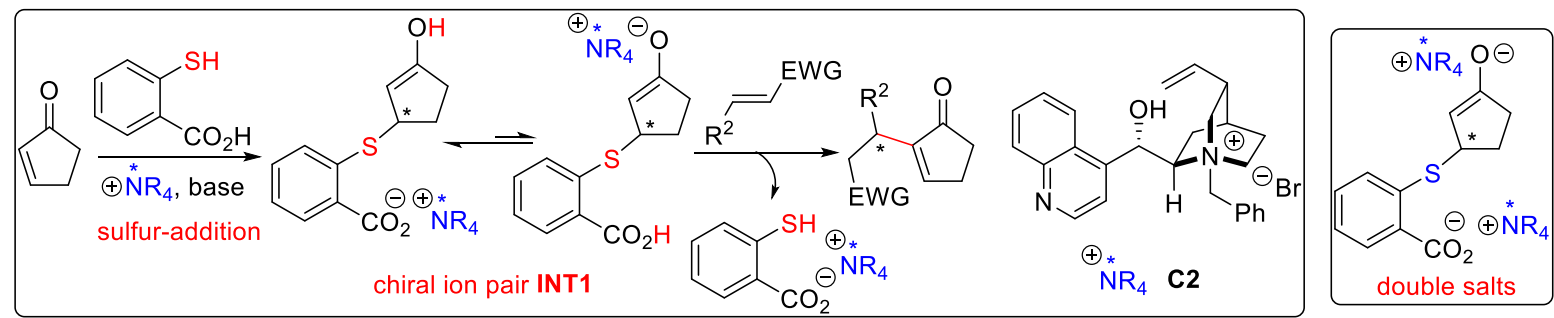

We have investigated the possible double salts after the early sulfur addition to enone 1a. However, we could not identify any stable intermediate assembled from enone 1a, thiol T1 and two equivalent of ammonium cation of $\mathbf{C 2}$. Therefore, it suggests that the double salt might be unstable and not be generated in the catalytic process. In contrast, we could successfully identify the complexes assembled from one equivalent of enone 1a, thiol T1 and PTC C2. As shown in the following Figure S2, the carboxylic anion might be better to combine with cation center of $\mathbf{C 2}$, because the energy of INT1-R-A is $8.9 \mathrm{kcal} / \mathrm{mol}$ higher that of INR1-R-B. We also tried different complex conformations, while their energies are higher than that of INR1-R-B. To explain the stereoselectivity, the pose employing the lowest energy of $S$-configuration (sulfide moiety) was calculated as INT1-S-B, whose energy was $2.0 \mathrm{kcal} / \mathrm{mol}$ lower than INT1-R-B, suggesting that the $\mathbf{C 2}$-catalyzed sulfur addition of T1 to enone 1a prefers to produce $S$-intermediate, which might be the key species for the subsequent stereoselective RC reaction. In addition, the enolate anion-complexed one INT1-S-A also has a higher 
energy than that of the carboxylic anion-related INT1-S-B. Nevertheless, INT1-S-A might be the more reliable active intermediate involved in the key $R C$ reaction.

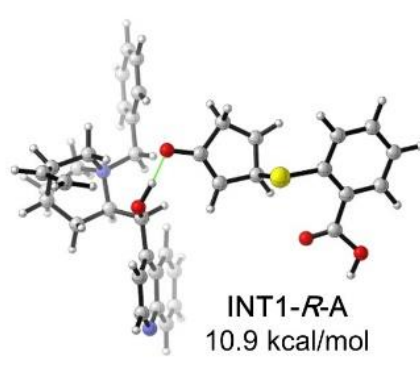

$10.9 \mathrm{kcal} / \mathrm{mol}$

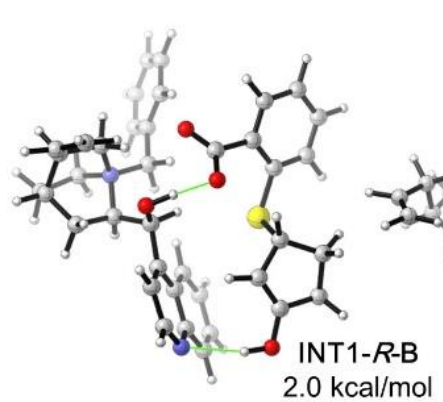

$2.0 \mathrm{kcal} / \mathrm{mol}$
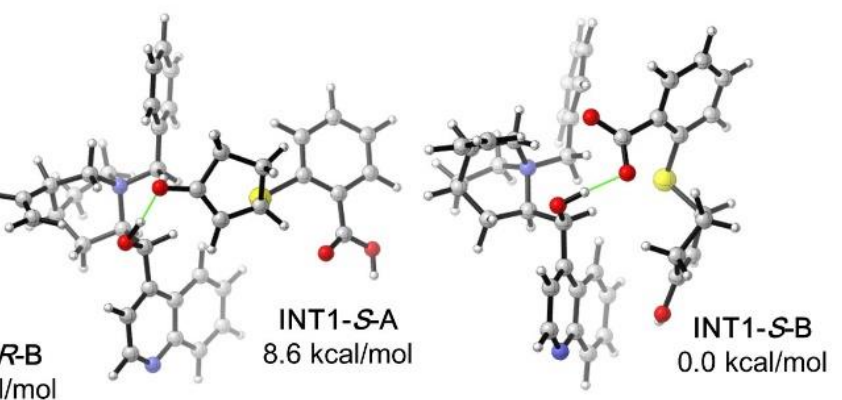

Figure S2. The structures and energies of the key ion pair INT1.

Since the subsequent addition of the chiral intermediate INT1-S-A to activated alkene $\mathbf{2}$ would generate the complicated intermediate with three stereogenic centers, and there are a lot of conformation possibilities for the ion pairs in the PTC-based reaction, we were unable to successfully conduct the DFT calculation studies on the following catalytic process [in fact, in comparison with well-established Lewis base catalysis (covalent bonding catalysis), there are very few examples involving DFT calculations of PTC catalysis; for a relatively simple asymmetric alkylation reaction, see: Petrova, G. P.; Li, H.-B.; Maruoka, K.; Morokuma, K. J. Phys. Chem. B 2014, 118, 5154]. As a result, based on the preliminary DFT calculations, a possible catalytic mechanism was proposed. As outlined in the following scheme, in the presence of PTC $\mathbf{C} 2$ and $\mathrm{K}_{2} \mathrm{CO}_{3}$, T1 attacks enone 1a from $\mathrm{Si}$-face, generating carboxylate ion pair intermediate $\mathbf{I}$. After proton transfer, the more reactive enolate ion pair intermediated II is formed, followed by another $S i$-face attack to acceptor $\mathbf{2 g}$. Then $\mathbf{3 g}$ is afforded after elimination of thiol T1, and then undergoes diastereoselective Michael addition and annulation with nitroolefin $\mathbf{4 a}$ to give final product $\mathbf{5 g}$, with the assistance of bases. 
<smiles>COc1ccc2c(c1)[C@@]1(C(=O)N2C)[C@H](c2ccccc2)[C@@H]([N+](=O)[O-])[C@@H]2CCC(=O)[C@H]21</smiles>

$5 g$

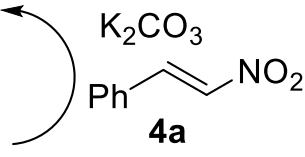

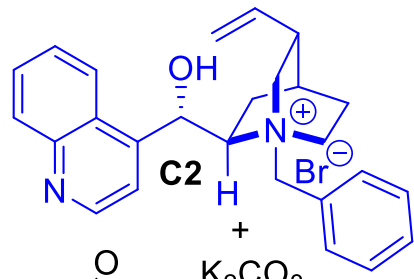

$\mathrm{MeO}$<smiles>N#CC(C#N)[C@]1(C2=CCCC2=O)C(=O)Nc2ccc(I)cc21</smiles>

3 g $\mathrm{CH}_{3}$

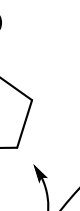<smiles>O=C(O)c1ccccc1S</smiles>

T1

C2

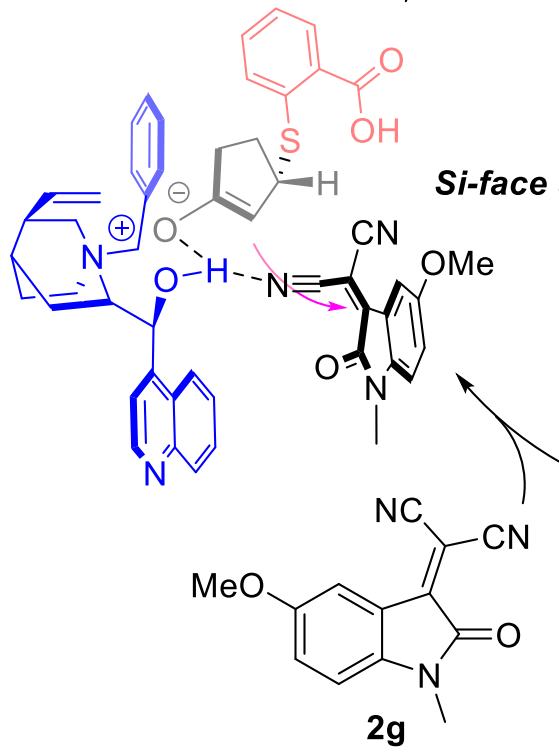

$2 \mathrm{~g}$

Proposed mechanism for the double activation catalysis

\section{Computational method:}

All calculations were carried out with the GAUSSIAN 09 packages. $^{2}$ The M06-2X functional, together with a basis set 6-31G(d), were used for optimizing the geometry of all the minima and transition states. All the optimized structures were confirmed by frequency calculations to minima states using the same level of theory. To take solvent effects into account, solution-phase single-point calculations were performed on the gas-phase geometries. ${ }^{3}$ The solution-phase single point energy were done using M06-2X method with a basis set 6-311++G(2d,p). Solvent effect was accounted for using self-consistent reaction field (SCRF) method, using SMD model and UAKS radii. ${ }^{4}$ Toluene was used as the solvent. Solution-phase single-point energies corrected by the gas-phase Gibbs free energy corrections were used to describe all the reaction energetics. All of these energies correspond 
to the reference state of $1 \mathrm{~mol} / \mathrm{L}, 298 \mathrm{~K}$. All energetics reported throughout the text are in $\mathrm{kcal} / \mathrm{mol}$. Structures were generated using GaussView5.0.8 and CYLview.

(2) Frisch, M. J.; Trucks, G. W.; Schlegel, H. B.; Scuseria, G. E.; Robb, M. A.; Cheeseman, J. R.; Scalmani, G.; Barone, V.; Mennucci, B.; Petersson, G. A.; Nakatsuji, H.; Caricato, M.; Li, X.; Hratchian, H. P.; Izmaylov, A. F.; Bloino, J.; Zheng, G.; Sonnenberg, J. L.; Hada, M.; Ehara, M.; Toyota, K.; Fukuda, R.; Hasegawa, J.; Ishida, M.; Nakajima, T.; Honda, Y.; Kitao, O.; Nakai, H.; Vreven, T.; Montgomery Jr., J. A.; Peralta, J. E.; Ogliaro, F.; Bearpark, M. J.; Heyd, J.; Brothers, E. N.; Kudin, K. N.; Staroverov, V. N.; Kobayashi, R.; Normand, J.; Raghavachari, K.; Rendell, A. P.; Burant, J. C.; Iyengar, S. S.; Tomasi, J.; Cossi, M.; Rega, N.; Millam, N. J.; Klene, M.; Knox, J. E.; Cross, J. B.; Bakken, V.; Adamo, C.; Jaramillo, J.; Gomperts, R.; Stratmann, R. E.; Yazyev, O.; Austin, A. J.; Cammi, R.; Pomelli, C.; Ochterski, J. W.; Martin, R. L.; Morokuma, K.; Zakrzewski, V. G.; Voth, G. A.; Salvador, P.; Dannenberg, J. J.; Dapprich, S.; Daniels, A. D.; Farkas, Ö.; Foresman, J. B.;

Ortiz, J. V.; Cioslowski, J.; Fox, D. J. Gaussian 09, Gaussian, Inc.: Wallingford, CT, USA, 2009.

(3) Um, J. M.; DiRocco, D. A.; Noey, E. L.; Rovis, T.; Houk, K. N. J. Am. Chem. Soc. 2011,133, 11249-11254.

(4) (a) Marenich, A. V.; Cramer, C. J.; Truhlar, D. G. J. Phys. Chem. B 2009,113, 6378-6396. (b) Ribeiro, R. F.; Marenich, A. V.; Cramer, C. J.; Truhlar, D. G. J. Phys. Chem. B 2011,115, 1455614562.

Computational data:

\section{C2-A}

\begin{tabular}{|c|c|c|c|c|c|}
\hline \multicolumn{3}{|c|}{ Zero-point correction= } & \multicolumn{3}{|c|}{0.507703 (Hartree/Particle) } \\
\hline \multicolumn{3}{|c|}{ Thermal correction to Energy= } & \multicolumn{3}{|c|}{0.531230} \\
\hline \multicolumn{3}{|c|}{ Thermal correction to Enthalpy= } & \multicolumn{3}{|c|}{0.532174} \\
\hline \multicolumn{3}{|c|}{ Thermal correction to Gibbs Free Energy= } & \multicolumn{3}{|c|}{0.454412} \\
\hline $\mathrm{E}(\mathrm{sov})=$ & -1192.63956 & A.U. & & & \\
\hline \multirow{2}{*}{$\begin{array}{l}\text { Center } \\
\text { Number }\end{array}$} & \multirow{2}{*}{$\begin{array}{l}\text { Atomic } \\
\text { Number }\end{array}$} & \multirow{2}{*}{$\begin{array}{l}\text { Atomic } \\
\text { Type }\end{array}$} & \multicolumn{3}{|c|}{ Coordinates (Angstroms) } \\
\hline & & & $\mathrm{X}$ & $\mathrm{Y}$ & Z \\
\hline 1 & 8 & 0 & 0.402282 & 1.218742 & -1.885185 \\
\hline 2 & 6 & 0 & 0.918140 & 0.210332 & -1.047973 \\
\hline 3 & 6 & 0 & 0.269875 & 0.254091 & 0.356339 \\
\hline
\end{tabular}




\begin{tabular}{|c|c|c|c|c|}
\hline 4 & 7 & -1.266854 & 0.152375 & 0.346896 \\
\hline 5 & 6 & -1.690912 & -0.199375 & 1.756048 \\
\hline 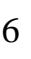 & 6 & -1.094652 & 0.809697 & 2.755348 \\
\hline y & 6 & -1.927513 & 1.468122 & -0.008969 \\
\hline 5 & 6 & -1.566714 & 2.560010 & 1.018434 \\
\hline & 6 & -1.105292 & 3.858716 & 0.399399 \\
\hline & 6 & -1.077396 & 4.199654 & -0.889478 \\
\hline & 6 & -0.505484 & 1.994415 & 1.984307 \\
\hline & 6 & 0.681217 & 1.499931 & 1.162062 \\
\hline & 6 & 2.427916 & 0.242901 & -0.875919 \\
\hline & 6 & 3.116462 & -0.899646 & -0.360822 \\
\hline & 6 & 2.504211 & -2.156562 & -0.096952 \\
\hline & 6 & 3.237168 & -3.206617 & 0.401069 \\
\hline & 6 & 4.619743 & -3.057821 & 0.665649 \\
\hline & 6 & 5.245113 & -1.865654 & 0.402371 \\
\hline & 6 & 4.515513 & -0.767882 & -0.124491 \\
\hline & 7 & 5.210331 & 0.374380 & -0.393398 \\
\hline & 6 & 4.555030 & 1.380219 & -0.911008 \\
\hline & 6 & 3.158668 & 1.365007 & -1.169574 \\
\hline & 6 & -3.161275 & -1.309987 & -0.567901 \\
\hline & 6 & -3.584748 & -2.360123 & 0.253764 \\
\hline & 6 & -4.925222 & -2.734643 & 0.286333 \\
\hline & 6 & -5.853715 & -2.068042 & -0.512410 \\
\hline & 6 & -5.437362 & -1.037105 & -1.353053 \\
\hline & 6 & -4.095579 & -0.664537 & -1.384511 \\
\hline & 6 & -1.705964 & -0.929084 & -0.633516 \\
\hline & 1 & 0.695737 & 2.095846 & -1.588588 \\
\hline & 1 & 0.660441 & -0.724224 & -1.553164 \\
\hline & 1 & 0.577552 & -0.648822 & 0.894430 \\
\hline & 1 & -2.782427 & -0.191226 & 1.754554 \\
\hline & 1 & -1.356737 & -1.223339 & 1.943568 \\
\hline & 1 & -0.313029 & 0.333780 & 3.355736 \\
\hline & 1 & -1.875840 & 1.142949 & 3.444235 \\
\hline 6 & 1 & -3.000273 & 1.268867 & -0.021785 \\
\hline & 1 & -1.598324 & 1.699976 & -1.019623 \\
\hline & 1 & -2.460824 & 2.789964 & 1.614776 \\
\hline & 1 & -0.780420 & 4.597645 & 1.133355 \\
\hline & 1 & -0.738934 & 5.187243 & -1.186635 \\
\hline & 1 & -1.412581 & 3.550702 & -1.695321 \\
\hline & 1 & -0.186358 & 2.776490 & 2.679518 \\
\hline & 1 & 1.529971 & 1.237906 & 1.800439 \\
\hline & 1 & 1.026531 & 2.301669 & 0.499133 \\
\hline & 1 & 1.452788 & -2.315617 & -0.324194 \\
\hline & 1 & 2.757241 & -4.164219 & 0.579174 \\
\hline & 1 & 5.183975 & -3.896693 & 1.060912 \\
\hline
\end{tabular}




$\begin{array}{rrrrrr}49 & 1 & 0 & 6.307420 & -1.721938 & 0.572910 \\ 50 & 1 & 0 & 5.131844 & 2.273461 & -1.141156 \\ 51 & 1 & 0 & 2.710081 & 2.250517 & -1.610831 \\ 52 & 1 & 0 & -2.861715 & -2.902897 & 0.859823 \\ 53 & 1 & 0 & -5.241807 & -3.555082 & 0.922603 \\ 54 & 1 & 0 & -6.897811 & -2.364611 & -0.493553 \\ 55 & 1 & 0 & -6.153032 & -0.533695 & -1.995449 \\ 56 & 1 & 0 & -3.770209 & 0.119254 & -2.066085 \\ 57 & 1 & 0 & -1.072176 & -1.795126 & -0.414794 \\ 58 & 1 & 0 & -1.457043 & -0.542099 & -1.624287\end{array}$

\section{C2-B}

Zero-point correction=

Thermal correction to Energy=

Thermal correction to Enthalpy=

Thermal correction to Gibbs Free Energy=

$\mathrm{E}(\mathrm{sov})=-1192.63566738 \quad$ A.U.

\author{
0.507985 (Hartree/Particle) \\ 0.531384 \\ 0.532328 \\ 0.455409
}

\begin{tabular}{rrrrrr} 
Center & Atomic & Atomic & \multicolumn{3}{c}{ Coordinates (Angstroms) } \\
Number & Number & Type & $\mathrm{X}$ & $\mathrm{Y}$ & $\mathrm{Z}$ \\
- & -1 & & & \\
\hline 1 & 8 & 0 & -0.029794 & 0.550276 & -2.968068 \\
2 & 6 & 0 & 0.792373 & -0.108816 & -2.024001 \\
3 & 6 & 0 & -0.065588 & -1.219149 & -1.357275 \\
4 & 7 & 0 & -1.253686 & -0.827260 & -0.460577 \\
5 & 6 & 0 & -2.164799 & -2.037618 & -0.381114 \\
6 & 6 & 0 & -1.362808 & -3.298567 & -0.030008 \\
7 & 6 & 0 & -0.763456 & -0.513105 & 0.936453 \\
8 & 6 & 0 & -0.263828 & -1.789544 & 1.635965 \\
9 & 6 & 0 & 0.962421 & -1.571516 & 2.488960 \\
10 & 6 & 0 & 1.656485 & -0.447420 & 2.664639 \\
11 & 6 & 0 & -0.018666 & -2.859687 & 0.549398 \\
12 & 6 & 0 & 0.805915 & -2.209102 & -0.559345 \\
13 & 6 & 0 & 1.538352 & 0.874732 & -1.123836 \\
14 & 6 & 0 & 2.831139 & 0.586194 & -0.577518 \\
15 & 6 & 0 & 3.619643 & -0.554584 & -0.903184 \\
16 & 6 & 0 & 4.843294 & -0.759651 & -0.316344 \\
17 & 6 & 0 & 5.352285 & 0.158145 & 0.635374 \\
18 & 6 & 0 & 4.638787 & 1.285942 & 0.946944 \\
19 & 6 & 0 & 3.381821 & 1.538025 & 0.334982 \\
20 & 7 & 0 & 2.764652 & 2.709907 & 0.654429 \\
21 & 6 & 0 & 1.639921 & 2.990626 & 0.046069
\end{tabular}




\begin{tabular}{|c|c|c|c|c|c|}
\hline 22 & 6 & 0 & 0.993275 & 2.110613 & -0.855998 \\
\hline 23 & 6 & 0 & -3.293327 & 0.714035 & -0.293329 \\
\hline 24 & 6 & 0 & -4.530322 & 0.157511 & -0.634590 \\
\hline 25 & 6 & 0 & -5.687988 & 0.541589 & 0.037670 \\
\hline 26 & 6 & 0 & -5.621320 & 1.496799 & 1.051326 \\
\hline 27 & 6 & 0 & -4.398116 & 2.078023 & 1.381009 \\
\hline 28 & 6 & 0 & -3.242449 & 1.691943 & 0.706631 \\
\hline 29 & 6 & 0 & -2.041380 & 0.342881 & -1.044452 \\
\hline 30 & 1 & 0 & 0.525498 & 1.062376 & -3.576018 \\
\hline 31 & 1 & 0 & 1.539405 & -0.711029 & -2.560990 \\
\hline 32 & 1 & 0 & -0.549402 & -1.724335 & -2.201151 \\
\hline 33 & 1 & 0 & -2.910707 & -1.795971 & 0.378790 \\
\hline 34 & 1 & 0 & -2.671418 & -2.115609 & -1.346415 \\
\hline 35 & 1 & 0 & -1.206151 & -3.919413 & -0.918304 \\
\hline 36 & 1 & 0 & -1.929285 & -3.896364 & 0.689669 \\
\hline 37 & 1 & 0 & -1.597877 & -0.061744 & 1.473669 \\
\hline 38 & 1 & 0 & 0.020595 & 0.234031 & 0.813508 \\
\hline 39 & 1 & 0 & -1.058791 & -2.163989 & 2.296773 \\
\hline 40 & 1 & 0 & 1.291028 & -2.470141 & 3.011711 \\
\hline 41 & 1 & 0 & 2.531257 & -0.437379 & 3.307349 \\
\hline 42 & 1 & 0 & 1.399797 & 0.505737 & 2.207754 \\
\hline 43 & 1 & 0 & 0.519291 & -3.711637 & 0.974677 \\
\hline 44 & 1 & 0 & 1.208221 & -2.954998 & -1.252937 \\
\hline 45 & 1 & 0 & 1.651158 & -1.692394 & -0.098709 \\
\hline 46 & 1 & 0 & 3.269901 & -1.273335 & -1.637675 \\
\hline 47 & 1 & 0 & 5.432087 & -1.629151 & -0.591941 \\
\hline 48 & 1 & 0 & 6.318263 & -0.024177 & 1.095735 \\
\hline 49 & 1 & 0 & 5.010169 & 2.028825 & 1.645785 \\
\hline 50 & 1 & 0 & 1.188245 & 3.954558 & 0.272965 \\
\hline 51 & 1 & 0 & 0.075104 & 2.434941 & -1.334391 \\
\hline 52 & 1 & 0 & -4.596089 & -0.567266 & -1.443579 \\
\hline 53 & 1 & 0 & -6.642992 & 0.105716 & -0.238837 \\
\hline 54 & 1 & 0 & -6.524345 & 1.800164 & 1.571946 \\
\hline 55 & 1 & 0 & -4.344380 & 2.839567 & 2.152819 \\
\hline 56 & 1 & 0 & -2.293321 & 2.168438 & 0.948552 \\
\hline 57 & 1 & 0 & -2.254188 & 0.064975 & -2.079113 \\
\hline 58 & 1 & 0 & -1.349488 & 1.179072 & -1.060614 \\
\hline
\end{tabular}

\section{C2-C}

Zero-point correction $=$

Thermal correction to Energy=

Thermal correction to Enthalpy=

Thermal correction to Gibbs Free Energy=
0.508352 (Hartree/Particle)

$$
0.531877
$$

0.532821

0.455801 
$\mathrm{E}(\mathrm{sov})=-1192.62657014 \quad$ A.U.

\begin{tabular}{|c|c|c|c|c|c|}
\hline \multirow{2}{*}{$\begin{array}{l}\text { Center } \\
\text { Number }\end{array}$} & \multirow{2}{*}{$\begin{array}{l}\text { Atomic } \\
\text { Number }\end{array}$} & \multirow{2}{*}{$\begin{array}{l}\text { Atomic } \\
\text { Type }\end{array}$} & \multicolumn{3}{|c|}{ Coordinates (Angstroms) } \\
\hline & & & $\mathrm{X}$ & $\mathrm{Y}$ & Z \\
\hline 1 & 8 & 0 & 0.033908 & 0.071354 & 1.097454 \\
\hline 2 & 6 & 0 & 0.302095 & -0.362925 & -0.227589 \\
\hline 3 & 6 & 0 & -0.954469 & -1.078632 & -0.728614 \\
\hline 4 & 7 & 0 & -2.157021 & -0.129099 & -0.928651 \\
\hline 5 & 6 & 0 & -3.136495 & -0.871164 & -1.827401 \\
\hline 6 & 6 & 0 & -3.392892 & -2.290580 & -1.299946 \\
\hline 7 & 6 & 0 & -2.866946 & 0.137097 & 0.385045 \\
\hline 8 & 6 & 0 & -3.548243 & -1.139758 & 0.910179 \\
\hline 9 & 6 & 0 & -3.461227 & -1.307280 & 2.408191 \\
\hline 10 & 6 & 0 & -2.566708 & -0.769161 & 3.236180 \\
\hline 11 & 6 & 0 & -2.940075 & -2.343962 & 0.161540 \\
\hline 12 & 6 & 0 & -1.417700 & -2.229050 & 0.190526 \\
\hline 13 & 6 & 0 & 1.579596 & -1.184086 & -0.318750 \\
\hline 14 & 6 & 0 & 2.800245 & -0.527881 & 0.051859 \\
\hline 15 & 6 & 0 & 2.891196 & 0.847195 & 0.413167 \\
\hline 16 & 6 & 0 & 4.098930 & 1.398862 & 0.770130 \\
\hline 17 & 6 & 0 & 5.276222 & 0.613842 & 0.785570 \\
\hline 18 & 6 & 0 & 5.225360 & -0.706408 & 0.416389 \\
\hline 19 & 6 & 0 & 3.996386 & -1.301452 & 0.031083 \\
\hline 20 & 7 & 0 & 4.030353 & -2.606242 & -0.365865 \\
\hline 21 & 6 & 0 & 2.910346 & -3.152059 & -0.757259 \\
\hline 22 & 6 & 0 & 1.656325 & -2.483398 & -0.750753 \\
\hline 23 & 6 & 0 & -1.167020 & 2.323149 & -0.926060 \\
\hline 24 & 6 & 0 & -1.819749 & 3.069877 & 0.061524 \\
\hline 25 & 6 & 0 & -1.197677 & 4.168451 & 0.648586 \\
\hline 26 & 6 & 0 & 0.072445 & 4.562543 & 0.232733 \\
\hline 27 & 6 & 0 & 0.709976 & 3.862465 & -0.789089 \\
\hline 28 & 6 & 0 & 0.091402 & 2.754544 & -1.362758 \\
\hline 29 & 6 & 0 & -1.836514 & 1.177901 & -1.659011 \\
\hline 30 & 1 & 0 & 0.862315 & 0.167755 & 1.592251 \\
\hline 31 & 1 & 0 & 0.471809 & 0.512641 & -0.860067 \\
\hline 32 & 1 & 0 & -0.745841 & -1.435401 & -1.745103 \\
\hline 33 & 1 & 0 & -4.047421 & -0.266868 & -1.830563 \\
\hline 34 & 1 & 0 & -2.713996 & -0.862214 & -2.835511 \\
\hline 35 & 1 & 0 & -2.845856 & -3.029825 & -1.894186 \\
\hline 36 & 1 & 0 & -4.458112 & -2.519875 & -1.395008 \\
\hline 37 & 1 & 0 & -3.610864 & 0.907787 & 0.173500 \\
\hline 38 & 1 & 0 & -2.115624 & 0.537788 & 1.061161 \\
\hline 39 & 1 & 0 & -4.614381 & -1.096731 & 0.649427 \\
\hline 40 & 1 & 0 & -4.211969 & -1.982328 & 2.817729 \\
\hline
\end{tabular}




$\begin{array}{lllrrc}41 & 1 & 0 & -2.596797 & -0.998358 & 4.296712 \\ 42 & 1 & 0 & -1.774071 & -0.101183 & 2.908961 \\ 43 & 1 & 0 & -3.259403 & -3.278209 & 0.631545 \\ 44 & 1 & 0 & -0.975503 & -3.166552 & -0.151246 \\ 45 & 1 & 0 & -1.061852 & -2.045924 & 1.208922 \\ 46 & 1 & 0 & 2.011836 & 1.487533 & 0.385373 \\ 47 & 1 & 0 & 4.150493 & 2.450160 & 1.037930 \\ 48 & 1 & 0 & 6.219452 & 1.064559 & 1.077909 \\ 49 & 1 & 0 & 6.110957 & -1.333539 & 0.396789 \\ 50 & 1 & 0 & 2.960646 & -4.186757 & -1.090339 \\ 51 & 1 & 0 & 0.787423 & -3.036494 & -1.087255 \\ 52 & 1 & 0 & -2.828447 & 2.821782 & 0.374192 \\ 53 & 1 & 0 & -1.717304 & 4.727848 & 1.420100 \\ 54 & 1 & 0 & 0.550836 & 5.425390 & 0.685572 \\ 55 & 1 & 0 & 1.684183 & 4.180186 & -1.148471 \\ 56 & 1 & 0 & 0.595749 & 2.229141 & -2.172261 \\ 57 & 1 & 0 & -2.808305 & 1.512810 & -2.035310 \\ 58 & 1 & 0 & -1.233388 & 0.882647 & -2.523616\end{array}$

\section{C2-D}

Zero-point correction=

Thermal correction to Energy=

Thermal correction to Enthalpy=

Thermal correction to Gibbs Free Energy=

$\mathrm{E}(\mathrm{sov})=-1192.62935513 \quad$ A.U.

\author{
0.507686 (Hartree/Particle) \\ 0.531191 \\ 0.532135 \\ 0.454731
}

\begin{tabular}{rrrrrr} 
Center & Atomic & Atomic & \multicolumn{3}{c}{ Coordinates (Angstroms) } \\
Number & Number & Type & X & Y & $Z$ \\
- & Z & & & \\
1 & 8 & 0 & 0.641701 & -0.203164 & 1.581305 \\
2 & 6 & 0 & -0.296269 & -0.263271 & 0.535310 \\
3 & 6 & 0 & 0.024273 & 0.751789 & -0.582502 \\
4 & 7 & 0 & 1.407695 & 0.577115 & -1.233243 \\
5 & 6 & 0 & 1.386105 & 1.397274 & -2.513204 \\
6 & 6 & 0 & 0.957993 & 2.846671 & -2.232155 \\
7 & 6 & 0 & 2.513120 & 1.134590 & -0.365498 \\
8 & 6 & 0 & 2.327769 & 2.649214 & -0.139288 \\
9 & 6 & 0 & 2.469818 & 3.077874 & 1.302476 \\
10 & 6 & 0 & 2.762039 & 2.329927 & 2.367004 \\
11 & 6 & 0 & 0.964103 & 3.077689 & -0.720550 \\
12 & 6 & 0 & -0.123252 & 2.209278 & -0.099399 \\
13 & 6 & 0 & -1.740576 & -0.052604 & 0.960614 \\
14 & 6 & 0 & -2.806751 & -0.452471 & 0.094640 \\
15 & 6 & 0 & -2.627645 & -1.158487 & -1.127621
\end{tabular}




\begin{tabular}{|c|c|c|c|c|c|}
\hline 16 & 6 & 0 & -3.704397 & -1.502715 & -1.908815 \\
\hline 17 & 6 & 0 & -5.019081 & -1.158385 & -1.512496 \\
\hline 18 & 6 & 0 & -5.228415 & -0.494694 & -0.330497 \\
\hline 19 & 6 & 0 & -4.135117 & -0.136447 & 0.501496 \\
\hline 20 & 7 & 0 & -4.422413 & 0.494001 & 1.675959 \\
\hline 21 & 6 & 0 & -3.425081 & 0.799239 & 2.464220 \\
\hline 22 & 6 & 0 & -2.062959 & 0.548268 & 2.150044 \\
\hline 23 & 6 & 0 & 2.194358 & -1.867981 & -0.645815 \\
\hline 24 & 6 & 0 & 3.498175 & -1.822596 & -0.138355 \\
\hline 25 & 6 & 0 & 3.951725 & -2.800241 & 0.741809 \\
\hline 26 & 6 & 0 & 3.118787 & -3.857790 & 1.102910 \\
\hline 27 & 6 & 0 & 1.840089 & -3.946665 & 0.558523 \\
\hline 28 & 6 & 0 & 1.387004 & -2.960928 & -0.313709 \\
\hline 29 & 6 & 0 & 1.709143 & -0.863939 & -1.666516 \\
\hline 30 & 1 & 0 & 0.516434 & 0.603333 & 2.106196 \\
\hline 31 & 1 & 0 & -0.206929 & -1.275855 & 0.147098 \\
\hline 32 & 1 & 0 & -0.666574 & 0.565941 & -1.411729 \\
\hline 33 & 1 & 0 & 2.401429 & 1.343244 & -2.914118 \\
\hline 34 & 1 & 0 & 0.715268 & 0.883918 & -3.206998 \\
\hline 35 & 1 & 0 & -0.042484 & 3.034590 & -2.633473 \\
\hline 36 & 1 & 0 & 1.645389 & 3.531871 & -2.736390 \\
\hline 37 & 1 & 0 & 3.443798 & 0.928219 & -0.898867 \\
\hline 38 & 1 & 0 & 2.495639 & 0.553885 & 0.552600 \\
\hline 39 & 1 & 0 & 3.103009 & 3.183369 & -0.706749 \\
\hline 40 & 1 & 0 & 2.325474 & 4.148902 & 1.450599 \\
\hline 41 & 1 & 0 & 2.852425 & 2.788581 & 3.346769 \\
\hline 42 & 1 & 0 & 2.951925 & 1.259974 & 2.323640 \\
\hline 43 & 1 & 0 & 0.783184 & 4.133242 & -0.497434 \\
\hline 44 & 1 & 0 & -1.121130 & 2.566387 & -0.370340 \\
\hline 45 & 1 & 0 & -0.056020 & 2.268290 & 0.992536 \\
\hline 46 & 1 & 0 & -1.633055 & -1.463934 & -1.444595 \\
\hline 47 & 1 & 0 & -3.548776 & -2.052817 & -2.832075 \\
\hline 48 & 1 & 0 & -5.859692 & -1.435119 & -2.140771 \\
\hline 49 & 1 & 0 & -6.223625 & -0.231234 & 0.013589 \\
\hline 50 & 1 & 0 & -3.676425 & 1.281581 & 3.406610 \\
\hline 51 & 1 & 0 & -1.310304 & 0.835053 & 2.879345 \\
\hline 52 & 1 & 0 & 4.183517 & -1.037079 & -0.443409 \\
\hline 53 & 1 & 0 & 4.963774 & -2.745237 & 1.130419 \\
\hline 54 & 1 & 0 & 3.475060 & -4.622453 & 1.785964 \\
\hline 55 & 1 & 0 & 1.197196 & -4.785724 & 0.804674 \\
\hline 56 & 1 & 0 & 0.396803 & -3.062230 & -0.755344 \\
\hline 57 & 1 & 0 & 2.466063 & -0.751956 & -2.448775 \\
\hline 58 & 1 & 0 & 0.788291 & -1.214819 & -2.144189 \\
\hline
\end{tabular}




\section{INT1-R-A}

Zero-point correction=

Thermal correction to Energy=

Thermal correction to Enthalpy=

Thermal correction to Gibbs Free Energy=
0.718649 (Hartree/Particle)

0.757193

0.758137

0.644645

$\mathrm{E}($ sov $)=-2280.52368238 \quad$ A.U.

\begin{tabular}{|c|c|c|c|c|c|}
\hline \multirow{2}{*}{$\begin{array}{l}\text { Center } \\
\text { Number }\end{array}$} & \multirow{2}{*}{$\begin{array}{l}\text { Atomic } \\
\text { Number }\end{array}$} & \multirow{2}{*}{$\begin{array}{l}\text { Atomic } \\
\text { Type }\end{array}$} & \multicolumn{3}{|c|}{ Coordinates (Angstroms) } \\
\hline & & & $\mathrm{X}$ & $\mathrm{Y}$ & Z \\
\hline 1 & 6 & 0 & -7.701189 & -0.134783 & -0.581587 \\
\hline 2 & 6 & 0 & -6.453435 & 0.166773 & -0.025738 \\
\hline 3 & 6 & 0 & -5.721666 & -0.828510 & 0.653182 \\
\hline 4 & 6 & 0 & -6.285896 & -2.106587 & 0.747871 \\
\hline 5 & 6 & 0 & -7.520522 & -2.403594 & 0.180509 \\
\hline 6 & 6 & 0 & -8.232208 & -1.414895 & -0.491762 \\
\hline 7 & 1 & 0 & -8.250731 & 0.651218 & -1.088018 \\
\hline 8 & 1 & 0 & -5.730893 & -2.870003 & 1.284416 \\
\hline 9 & 1 & 0 & -7.926693 & -3.407046 & 0.268106 \\
\hline 10 & 1 & 0 & -9.197350 & -1.634479 & -0.937168 \\
\hline 11 & 16 & 0 & -4.168585 & -0.549373 & 1.489658 \\
\hline 12 & 6 & 0 & -5.941983 & 1.559230 & -0.193989 \\
\hline 13 & 8 & 0 & -4.790899 & 1.898873 & -0.305787 \\
\hline 14 & 6 & 0 & -2.945954 & -0.591257 & 0.070155 \\
\hline 15 & 6 & 0 & -2.795582 & -2.010111 & -0.505528 \\
\hline 16 & 6 & 0 & -1.593836 & -0.301747 & 0.630850 \\
\hline 17 & 1 & 0 & -3.322995 & 0.139168 & -0.646804 \\
\hline 18 & 6 & 0 & -1.683032 & -2.649188 & 0.333920 \\
\hline 19 & 1 & 0 & -2.457925 & -1.913405 & -1.544801 \\
\hline 20 & 1 & 0 & -3.733367 & -2.573983 & -0.511374 \\
\hline 21 & 6 & 0 & -0.882642 & -1.447630 & 0.817970 \\
\hline 22 & 1 & 0 & -1.294436 & 0.680431 & 0.982626 \\
\hline 23 & 1 & 0 & -2.080660 & -3.189395 & 1.203012 \\
\hline 24 & 1 & 0 & -1.061878 & -3.352903 & -0.232739 \\
\hline 25 & 8 & 0 & 0.315290 & -1.613364 & 1.318940 \\
\hline 26 & 8 & 0 & 1.379731 & 0.563378 & 1.764584 \\
\hline 27 & 6 & 0 & 1.590415 & 1.039658 & 0.481519 \\
\hline 28 & 6 & 0 & 3.035867 & 0.728050 & -0.009915 \\
\hline 29 & 7 & 0 & 3.329635 & -0.765443 & -0.242906 \\
\hline 30 & 6 & 0 & 4.608467 & -0.853056 & -1.037295 \\
\hline 31 & 6 & 0 & 5.709519 & 0.001880 & -0.389418 \\
\hline 32 & 6 & 0 & 3.544418 & -1.470310 & 1.080384 \\
\hline 33 & 6 & 0 & 4.854412 & -0.986439 & 1.734207 \\
\hline 34 & 6 & 0 & 4.764340 & -0.832238 & 3.234175 \\
\hline
\end{tabular}




\begin{tabular}{|c|c|c|c|c|c|}
\hline 35 & 6 & 0 & 3.684121 & -0.503200 & 3.938556 \\
\hline 36 & 6 & 0 & 5.282352 & 0.329412 & 1.043416 \\
\hline 37 & 6 & 0 & 4.080504 & 1.269361 & 0.982447 \\
\hline 38 & 6 & 0 & 1.445494 & 2.555070 & 0.433547 \\
\hline 39 & 6 & 0 & 1.369257 & 3.274481 & -0.798663 \\
\hline 40 & 6 & 0 & 1.297170 & 2.659235 & -2.077989 \\
\hline 41 & 6 & 0 & 1.233513 & 3.414627 & -3.222055 \\
\hline 42 & 6 & 0 & 1.238648 & 4.827147 & -3.149500 \\
\hline 43 & 6 & 0 & 1.282200 & 5.451113 & -1.929998 \\
\hline 44 & 6 & 0 & 1.336465 & 4.695861 & -0.729861 \\
\hline 45 & 7 & 0 & 1.346748 & 5.386802 & 0.446232 \\
\hline 46 & 6 & 0 & 1.365605 & 4.684917 & 1.552062 \\
\hline 47 & 6 & 0 & 1.417169 & 3.269911 & 1.602246 \\
\hline 48 & 6 & 0 & 2.407820 & -2.849604 & -1.391209 \\
\hline 49 & 6 & 0 & 3.037609 & -3.228667 & -2.579603 \\
\hline 50 & 6 & 0 & 3.188615 & -4.574750 & -2.898258 \\
\hline 51 & 6 & 0 & 2.701488 & -5.552096 & -2.033100 \\
\hline 52 & 6 & 0 & 2.052402 & -5.181359 & -0.857657 \\
\hline 53 & 6 & 0 & 1.898499 & -3.835185 & -0.537369 \\
\hline 54 & 6 & 0 & 2.191572 & -1.404654 & -1.026836 \\
\hline 55 & 1 & 0 & 0.845458 & -0.354945 & 1.671937 \\
\hline 56 & 1 & 0 & 0.853428 & 0.614947 & -0.217236 \\
\hline 57 & 1 & 0 & 3.179156 & 1.168652 & -1.003138 \\
\hline 58 & 1 & 0 & 4.870892 & -1.912672 & -1.070614 \\
\hline 59 & 1 & 0 & 4.372587 & -0.527703 & -2.054040 \\
\hline 60 & 1 & 0 & 5.860639 & 0.926364 & -0.955979 \\
\hline 61 & 1 & 0 & 6.652384 & -0.552559 & -0.404881 \\
\hline 62 & 1 & 0 & 3.585671 & -2.537416 & 0.860060 \\
\hline 63 & 1 & 0 & 2.658145 & -1.269691 & 1.676194 \\
\hline 64 & 1 & 0 & 5.637281 & -1.728471 & 1.526915 \\
\hline 65 & 1 & 0 & 5.712124 & -0.969944 & 3.753387 \\
\hline 66 & 1 & 0 & 3.751270 & -0.387114 & 5.015970 \\
\hline 67 & 1 & 0 & 2.713172 & -0.318194 & 3.484791 \\
\hline 68 & 1 & 0 & 6.105505 & 0.791294 & 1.596194 \\
\hline 69 & 1 & 0 & 4.382244 & 2.270963 & 0.662667 \\
\hline 70 & 1 & 0 & 3.617891 & 1.355852 & 1.970344 \\
\hline 71 & 1 & 0 & 1.252352 & 1.576001 & -2.151087 \\
\hline 72 & 1 & 0 & 1.162087 & 2.926838 & -4.189421 \\
\hline 73 & 1 & 0 & 1.190833 & 5.412324 & -4.062561 \\
\hline 74 & 1 & 0 & 1.265950 & 6.531832 & -1.833550 \\
\hline 75 & 1 & 0 & 1.352008 & 5.250860 & 2.482289 \\
\hline 76 & 1 & 0 & 1.431268 & 2.743128 & 2.549464 \\
\hline 77 & 1 & 0 & 3.398971 & -2.468014 & -3.268688 \\
\hline 78 & 1 & 0 & 3.676643 & -4.860362 & -3.824922 \\
\hline 79 & 1 & 0 & 2.816053 & -6.602387 & -2.283137 \\
\hline
\end{tabular}




$\begin{array}{lllrrc}80 & 1 & 0 & 1.653077 & -5.940550 & -0.192548 \\ 81 & 1 & 0 & 1.364894 & -3.520272 & 0.360063 \\ 82 & 1 & 0 & 2.068684 & -0.777007 & -1.916412 \\ 83 & 1 & 0 & 1.316872 & -1.339352 & -0.375906 \\ 84 & 8 & 0 & -6.950027 & 2.461771 & -0.245942 \\ 85 & 1 & 0 & -6.520687 & 3.321975 & -0.393678\end{array}$

\section{INT1-S-B}

\begin{tabular}{|c|c|c|c|c|c|}
\hline \multicolumn{3}{|c|}{ Zero-point correction= } & \multicolumn{3}{|c|}{0.720418 (Hartree/Particle) } \\
\hline Thermal c & rrection to $\mathrm{E}$ & $g y=$ & \multicolumn{3}{|c|}{0.758590} \\
\hline Thermal c & rrection to $\mathrm{E}$ & alpy $=$ & \multicolumn{3}{|c|}{0.759534} \\
\hline \multicolumn{3}{|c|}{ Thermal correction to Gibbs Free Energy= } & \multicolumn{3}{|c|}{0.649767} \\
\hline $\mathrm{E}(\mathrm{sov})=$ & -2280.54312 & A.U. & \\
\hline \multirow{2}{*}{$\begin{array}{l}\text { Center } \\
\text { Number }\end{array}$} & \multirow{2}{*}{$\begin{array}{l}\text { Atomic } \\
\text { Number }\end{array}$} & \multirow{2}{*}{$\begin{array}{l}\text { Atomic } \\
\text { Type }\end{array}$} & \multicolumn{3}{|c|}{ Coordinates (Angstroms) } \\
\hline & & & $\mathrm{X}$ & $\mathrm{Y}$ & Z \\
\hline 1 & 6 & 0 & 0.931409 & -4.110948 & 1.583840 \\
\hline 2 & 6 & 0 & 0.146078 & -3.017546 & 1.213301 \\
\hline 3 & 6 & 0 & -0.876935 & -3.198953 & 0.265589 \\
\hline 4 & 6 & 0 & -1.096323 & -4.472556 & -0.269202 \\
\hline 5 & 6 & 0 & -0.328700 & -5.560575 & 0.135252 \\
\hline 6 & 6 & 0 & 0.690189 & -5.378709 & 1.066236 \\
\hline 7 & 1 & 0 & 1.738263 & -3.944003 & 2.291601 \\
\hline 8 & 1 & 0 & -1.873409 & -4.597139 & -1.017548 \\
\hline 9 & 1 & 0 & -0.519723 & -6.543761 & -0.284693 \\
\hline 10 & 1 & 0 & 1.300807 & -6.220409 & 1.379688 \\
\hline 11 & 16 & 0 & -1.840827 & -1.830890 & -0.373343 \\
\hline 12 & 6 & 0 & 0.475881 & -1.669273 & 1.831064 \\
\hline 13 & 8 & 0 & 1.682468 & -1.323377 & 1.811328 \\
\hline 14 & 6 & 0 & -3.146399 & -1.676915 & 0.950783 \\
\hline 15 & 6 & 0 & -4.351843 & -2.596439 & 0.673711 \\
\hline 16 & 6 & 0 & -3.749793 & -0.306615 & 0.896578 \\
\hline 17 & 1 & 0 & -2.614649 & -1.886634 & 1.879759 \\
\hline 18 & 6 & 0 & -5.318680 & -1.734600 & -0.157181 \\
\hline 19 & 1 & 0 & -4.821283 & -2.834649 & 1.635037 \\
\hline 20 & 1 & 0 & -4.069546 & -3.535786 & 0.191412 \\
\hline 21 & 6 & 0 & -4.933867 & -0.341165 & 0.268943 \\
\hline 22 & 1 & 0 & -3.248376 & 0.570776 & 1.285796 \\
\hline 23 & 1 & 0 & -5.158616 & -1.850997 & -1.236755 \\
\hline 24 & 1 & 0 & -6.373253 & -1.944845 & 0.045514 \\
\hline 25 & 8 & 0 & -5.788290 & 0.659427 & -0.030166 \\
\hline
\end{tabular}


60

61

62

63

64

65

66

67

68

69

70

$\begin{array}{rrr}0.331170 & 1.378699 & 1.861097 \\ 0.026221 & 1.402624 & 0.504836 \\ 1.149987 & 2.148687 & -0.248449 \\ 2.437644 & 1.322966 & -0.449883 \\ 3.316500 & 2.081266 & -1.411932 \\ 3.530538 & 3.528985 & -0.937632 \\ 3.174049 & 1.167277 & 0.863203 \\ 3.686058 & 2.535808 & 1.348703 \\ 3.496699 & 2.765477 & 2.827951 \\ 2.862406 & 1.985412 & 3.698812 \\ 3.026909 & 3.646222 & 0.500999 \\ 1.513819 & 3.451823 & 0.494181 \\ -1.307305 & 2.099190 & 0.241477 \\ -2.025567 & 1.917606 & -0.978845 \\ -1.534242 & 1.182499 & -2.089588 \\ -2.301468 & 0.998900 & -3.211104 \\ -3.608085 & 1.535197 & -3.281669 \\ -4.122064 & 2.233630 & -2.221149 \\ -3.346789 & 2.438530 & -1.051009 \\ -3.942521 & 3.093672 & -0.009929 \\ -3.225975 & 3.288367 & 1.073434 \\ -1.904271 & 2.816230 & 1.247198 \\ 3.287539 & -0.886850 & -1.434160 \\ 3.851117 & -0.788655 & -2.710209 \\ 4.915593 & -1.605261 & -3.076098 \\ 5.416307 & -2.538392 & -2.170092 \\ 4.840946 & -2.660735 & -0.908209 \\ 3.774664 & -1.844508 & -0.537763 \\ 2.102673 & -0.046562 & -1.030279 \\ 0.013254 & 0.474955 & 2.211526 \\ -0.104901 & 0.382719 & 0.118339 \\ 0.823156 & 2.355851 & -1.274058 \\ 4.252793 & 1.523573 & -1.464893 \\ 2.832527 & 2.029402 & -2.391398 \\ 2.986221 & 4.226403 & -1.582263 \\ 4.593520 & 3.778329 & -1.005583 \\ 3.995693 & 0.475165 & 0.668938 \\ 2.467757 & 0.682700 & 1.536378 \\ 4.767076 & 2.593456 & 1.150440 \\ 3.951655 & 3.690278 & 3.185537 \\ 2.806624 & 2.277012 & 4.743240 \\ 1.1471789 & 3.382127 & 1.521024\end{array}$




$\begin{array}{rrrrrr}71 & 1 & 0 & -0.557667 & 0.715727 & -2.031896 \\ 72 & 1 & 0 & -1.913761 & 0.417425 & -4.041661 \\ 73 & 1 & 0 & -4.207708 & 1.373572 & -4.171912 \\ 74 & 1 & 0 & -5.130682 & 2.633656 & -2.230263 \\ 75 & 1 & 0 & -3.719987 & 3.813274 & 1.889165 \\ 76 & 1 & 0 & -1.395444 & 2.932254 & 2.197366 \\ 77 & 1 & 0 & 3.445580 & -0.080127 & -3.429628 \\ 78 & 1 & 0 & 5.345316 & -1.522067 & -4.069457 \\ 79 & 1 & 0 & 6.244693 & -3.179405 & -2.455802 \\ 80 & 1 & 0 & 5.213871 & -3.404011 & -0.210595 \\ 81 & 1 & 0 & 3.286449 & -1.950928 & 0.431270 \\ 82 & 1 & 0 & 1.455846 & 0.155381 & -1.889730 \\ 83 & 1 & 0 & 1.551214 & -0.572773 & -0.251778 \\ 84 & 8 & 0 & -0.479882 & -0.996846 & 2.301471 \\ 85 & 1 & 0 & -5.384978 & 1.516444 & 0.202901\end{array}$

\section{INT1-R-A}

\begin{tabular}{|c|c|c|c|c|c|}
\hline \multicolumn{3}{|c|}{ Zero-point correction= } & \multicolumn{3}{|c|}{0.719391 (Hartree/Particle) } \\
\hline \multicolumn{3}{|c|}{ Thermal correction to Energy= } & \multicolumn{3}{|c|}{0.757813} \\
\hline \multicolumn{3}{|c|}{ Thermal correction to Enthalpy= } & \multicolumn{3}{|c|}{0.758758} \\
\hline \multicolumn{3}{|c|}{ Thermal correction to Gibbs Free Energy= } & \multicolumn{3}{|c|}{0.648548} \\
\hline \multicolumn{3}{|c|}{$E($ sov $)=-2280.54501531 \quad$ A.U. } & & & \\
\hline \multirow{2}{*}{$\begin{array}{l}\text { Center } \\
\text { Number }\end{array}$} & \multirow{2}{*}{$\begin{array}{l}\text { Atomic } \\
\text { Number }\end{array}$} & \multirow{2}{*}{$\begin{array}{l}\text { Atomic } \\
\text { Type }\end{array}$} & \multicolumn{3}{|c|}{ Coordinates (Angstroms) } \\
\hline & & & $\mathrm{X}$ & $\mathrm{Y}$ & Z \\
\hline 1 & 6 & 0 & 3.785533 & 2.287987 & -1.927502 \\
\hline 2 & 6 & 0 & 2.554484 & 2.029567 & -1.317723 \\
\hline 3 & 6 & 0 & 2.177526 & 2.787888 & -0.198157 \\
\hline 4 & 6 & 0 & 3.020673 & 3.806851 & 0.259566 \\
\hline 5 & 6 & 0 & 4.214976 & 4.090606 & -0.392254 \\
\hline 6 & 6 & 0 & 4.602406 & 3.321828 & -1.488235 \\
\hline 7 & 1 & 0 & 4.083071 & 1.662501 & -2.763868 \\
\hline 8 & 1 & 0 & 2.732694 & 4.367926 & 1.144258 \\
\hline 9 & 1 & 0 & 4.850894 & 4.894082 & -0.032775 \\
\hline 10 & 1 & 0 & 5.543786 & 3.522509 & -1.991195 \\
\hline 11 & 16 & 0 & 0.686735 & 2.438447 & 0.731489 \\
\hline 12 & 6 & 0 & 1.717556 & 0.898685 & -1.897914 \\
\hline 13 & 8 & 0 & 2.287450 & -0.208618 & -2.015567 \\
\hline 14 & 6 & 0 & -0.420470 & 3.841361 & 0.220499 \\
\hline 15 & 6 & 0 & -0.721414 & 3.871334 & -1.290624 \\
\hline 16 & 6 & 0 & -1.765345 & 3.604273 & 0.844286 \\
\hline 17 & 1 & 0 & 0.077312 & 4.752905 & 0.564445 \\
\hline
\end{tabular}




\begin{tabular}{|c|c|c|c|c|c|}
\hline 18 & 6 & 0 & -2.015720 & 3.063942 & -1.453397 \\
\hline 19 & 1 & 0 & 0.094702 & 3.475513 & -1.895679 \\
\hline 20 & 1 & 0 & -0.902898 & 4.916103 & -1.567553 \\
\hline 21 & 6 & 0 & -2.626894 & 3.162256 & -0.083896 \\
\hline 22 & 1 & 0 & -1.965282 & 3.698214 & 1.906775 \\
\hline 23 & 1 & 0 & -2.683366 & 3.456779 & -2.226218 \\
\hline 24 & 1 & 0 & -1.804097 & 2.014470 & -1.697852 \\
\hline 25 & 8 & 0 & -3.912454 & 2.779652 & 0.052512 \\
\hline 26 & 8 & 0 & -0.749566 & -1.026741 & -1.974249 \\
\hline 27 & 6 & 0 & -1.178394 & -0.830878 & -0.665707 \\
\hline 28 & 6 & 0 & -0.844051 & -2.084075 & 0.186519 \\
\hline 29 & 7 & 0 & 0.642964 & -2.257595 & 0.540160 \\
\hline 30 & 6 & 0 & 0.728394 & -3.298507 & 1.624647 \\
\hline 31 & 6 & 0 & -0.027846 & -4.572128 & 1.209129 \\
\hline 32 & 6 & 0 & 1.420467 & -2.743612 & -0.663165 \\
\hline 33 & 6 & 0 & 0.990430 & -4.170478 & -1.036542 \\
\hline 34 & 6 & 0 & 0.861858 & -4.378692 & -2.525689 \\
\hline 35 & 6 & 0 & 1.014982 & -3.470106 & -3.485673 \\
\hline 36 & 6 & 0 & -0.321556 & -4.495978 & -0.290904 \\
\hline 37 & 6 & 0 & -1.319539 & -3.364883 & -0.528476 \\
\hline 38 & 6 & 0 & -2.692407 & -0.660159 & -0.584844 \\
\hline 39 & 6 & 0 & -3.336542 & -0.165399 & 0.589781 \\
\hline 40 & 6 & 0 & -2.638654 & 0.339095 & 1.719676 \\
\hline 41 & 6 & 0 & -3.320268 & 0.842779 & 2.801272 \\
\hline 42 & 6 & 0 & -4.736269 & 0.857936 & 2.812757 \\
\hline 43 & 6 & 0 & -5.437972 & 0.383905 & 1.731765 \\
\hline 44 & 6 & 0 & -4.759222 & -0.124189 & 0.594209 \\
\hline 45 & 7 & 0 & -5.521108 & -0.524349 & -0.462750 \\
\hline 46 & 6 & 0 & -4.887567 & -0.927601 & -1.535908 \\
\hline 47 & 6 & 0 & -3.477676 & -1.011993 & -1.650819 \\
\hline 48 & 6 & 0 & 2.591963 & -0.994841 & 1.623844 \\
\hline 49 & 6 & 0 & 2.769327 & -1.221548 & 2.992197 \\
\hline 50 & 6 & 0 & 4.044070 & -1.230762 & 3.547097 \\
\hline 51 & 6 & 0 & 5.153340 & -0.996226 & 2.737135 \\
\hline 52 & 6 & 0 & 4.981877 & -0.743840 & 1.378951 \\
\hline 53 & 6 & 0 & 3.706717 & -0.738017 & 0.818116 \\
\hline 54 & 6 & 0 & 1.210743 & -0.935509 & 1.025818 \\
\hline 55 & 1 & 0 & -0.247761 & -0.183384 & -2.246520 \\
\hline 56 & 1 & 0 & -0.715337 & 0.072845 & -0.243263 \\
\hline 57 & 1 & 0 & -1.322049 & -1.978015 & 1.167558 \\
\hline 58 & 1 & 0 & 1.792924 & -3.480379 & 1.784305 \\
\hline 59 & 1 & 0 & 0.319831 & -2.848211 & 2.533617 \\
\hline 60 & 1 & 0 & -0.964786 & -4.659711 & 1.768445 \\
\hline 61 & 1 & 0 & 0.581932 & -5.448162 & 1.448430 \\
\hline 62 & 1 & 0 & 2.475748 & -2.692311 & -0.389301 \\
\hline
\end{tabular}




$\begin{array}{lllrrr}63 & 1 & 0 & 1.236526 & -2.007118 & -1.442388 \\ 64 & 1 & 0 & 1.749313 & -4.878726 & -0.671077 \\ 65 & 1 & 0 & 0.615970 & -5.404361 & -2.804268 \\ 66 & 1 & 0 & 0.892466 & -3.758341 & -4.525367 \\ 67 & 1 & 0 & 1.265809 & -2.428629 & -3.300500 \\ 68 & 1 & 0 & -0.727661 & -5.446508 & -0.650391 \\ 69 & 1 & 0 & -2.311966 & -3.631922 & -0.153845 \\ 70 & 1 & 0 & -1.396726 & -3.158924 & -1.599799 \\ 71 & 1 & 0 & -1.553290 & 0.391998 & 1.704164 \\ 72 & 1 & 0 & -2.770006 & 1.246665 & 3.645958 \\ 73 & 1 & 0 & -5.262714 & 1.253511 & 3.676268 \\ 74 & 1 & 0 & -6.522480 & 0.396276 & 1.697321 \\ 75 & 1 & 0 & -5.508842 & -1.222533 & -2.380145 \\ 76 & 1 & 0 & -3.010434 & -1.348229 & -2.569887 \\ 77 & 1 & 0 & 1.902615 & -1.373815 & 3.632405 \\ 78 & 1 & 0 & 4.170976 & -1.404961 & 4.611044 \\ 79 & 1 & 0 & 6.149226 & -0.993862 & 3.169875 \\ 80 & 1 & 0 & 5.842012 & -0.534091 & 0.750936 \\ 81 & 1 & 0 & 3.558892 & -0.519631 & -0.240228 \\ 82 & 1 & 0 & 0.486949 & -0.552446 & 1.752516 \\ 83 & 1 & 0 & 1.215861 & -0.285692 & 0.153837 \\ 84 & 8 & 0 & 0.525109 & 1.166855 & -2.207761 \\ 85 & 1 & 0 & -4.131974 & 2.701710 & 0.995793\end{array}$

\section{INT1-S-B}

Zero-point correction=

Thermal correction to Energy=

Thermal correction to Enthalpy=

Thermal correction to Gibbs Free Energy=

\author{
0.718072 (Hartree/Particle) \\ 0.756800 \\ 0.757745
}

0.643008

\begin{tabular}{|c|c|c|c|c|c|}
\hline \multirow{2}{*}{$\begin{array}{l}\text { Center } \\
\text { Number }\end{array}$} & \multirow{2}{*}{$\begin{array}{l}\text { Atomic } \\
\text { Number }\end{array}$} & \multirow{2}{*}{$\begin{array}{l}\text { Atomic } \\
\text { Type }\end{array}$} & \multicolumn{3}{|c|}{ Coordinates (Angstroms) } \\
\hline & & & $\mathrm{X}$ & $\mathrm{Y}$ & Z \\
\hline 1 & 6 & 0 & 6.527930 & 0.057991 & -0.600615 \\
\hline 2 & 6 & 0 & 5.278326 & -0.414885 & -0.184622 \\
\hline 3 & 6 & 0 & 4.153626 & 0.433627 & -0.226602 \\
\hline 4 & 6 & 0 & 4.334028 & 1.743501 & -0.689149 \\
\hline 5 & 6 & 0 & 5.580827 & 2.208797 & -1.091861 \\
\hline 6 & 6 & 0 & 6.685811 & 1.363952 & -1.045460 \\
\hline 7 & 1 & 0 & 7.377972 & -0.614665 & -0.569344 \\
\hline 8 & 1 & 0 & 3.465758 & 2.395442 & -0.723897 \\
\hline 9 & 1 & 0 & 5.687958 & 3.231787 & -1.440461 \\
\hline
\end{tabular}




\begin{tabular}{|c|c|c|c|c|c|}
\hline 10 & 1 & 0 & 7.663727 & 1.716822 & -1.356805 \\
\hline 11 & 16 & 0 & 2.483165 & -0.054879 & 0.171024 \\
\hline 12 & 6 & 0 & 5.192539 & -1.822303 & 0.303248 \\
\hline 13 & 8 & 0 & 4.413706 & -2.258389 & 1.113106 \\
\hline 14 & 6 & 0 & 2.386466 & -0.073115 & 2.068136 \\
\hline 15 & 6 & 0 & 2.693972 & 1.318051 & 2.635161 \\
\hline 16 & 6 & 0 & 0.947222 & -0.269832 & 2.383784 \\
\hline 17 & 1 & 0 & 3.076561 & -0.857784 & 2.376024 \\
\hline 18 & 6 & 0 & 1.356860 & 2.056717 & 2.533305 \\
\hline 19 & 1 & 0 & 3.523316 & 1.821899 & 2.128797 \\
\hline 20 & 1 & 0 & 2.969041 & 1.187139 & 3.688285 \\
\hline 21 & 6 & 0 & 0.321209 & 0.934620 & 2.561580 \\
\hline 22 & 1 & 0 & 0.457848 & -1.237972 & 2.363704 \\
\hline 23 & 1 & 0 & 1.180051 & 2.779770 & 3.336047 \\
\hline 24 & 1 & 0 & 1.281499 & 2.600428 & 1.580653 \\
\hline 25 & 8 & 0 & -0.941674 & 1.223691 & 2.644650 \\
\hline 26 & 8 & 0 & -2.463582 & -0.571405 & 1.811711 \\
\hline 27 & 6 & 0 & -1.846787 & -1.006225 & 0.644330 \\
\hline 28 & 6 & 0 & -2.527654 & -0.412702 & -0.617976 \\
\hline 29 & 7 & 0 & -2.367385 & 1.111665 & -0.762744 \\
\hline 30 & 6 & 0 & -2.727557 & 1.469558 & -2.180319 \\
\hline 31 & 6 & 0 & -4.080726 & 0.847676 & -2.564250 \\
\hline 32 & 6 & 0 & -3.323044 & 1.825524 & 0.169198 \\
\hline 33 & 6 & 0 & -4.778098 & 1.613758 & -0.293610 \\
\hline 34 & 6 & 0 & -5.753808 & 1.424003 & 0.843738 \\
\hline 35 & 6 & 0 & -5.498803 & 0.872627 & 2.028260 \\
\hline 36 & 6 & 0 & -4.799453 & 0.422618 & -1.280157 \\
\hline 37 & 6 & 0 & -4.029377 & -0.740066 & -0.659259 \\
\hline 38 & 6 & 0 & -1.935423 & -2.518129 & 0.502751 \\
\hline 39 & 6 & 0 & -1.121029 & -3.233106 & -0.426753 \\
\hline 40 & 6 & 0 & -0.076625 & -2.637154 & -1.184155 \\
\hline 41 & 6 & 0 & 0.671803 & -3.385723 & -2.057219 \\
\hline 42 & 6 & 0 & 0.410204 & -4.765803 & -2.225236 \\
\hline 43 & 6 & 0 & -0.576226 & -5.373961 & -1.492712 \\
\hline 44 & 6 & 0 & -1.354107 & -4.630144 & -0.567920 \\
\hline 45 & 7 & 0 & -2.291814 & -5.305967 & 0.157026 \\
\hline 46 & 6 & 0 & -2.977539 & -4.619337 & 1.037814 \\
\hline 47 & 6 & 0 & -2.839460 & -3.225635 & 1.251622 \\
\hline 48 & 6 & 0 & -0.621290 & 2.965884 & -0.700300 \\
\hline 49 & 6 & 0 & -0.157558 & 3.432440 & -1.932909 \\
\hline 50 & 6 & 0 & 0.146189 & 4.779560 & -2.107423 \\
\hline 51 & 6 & 0 & -0.003392 & 5.668723 & -1.045561 \\
\hline 52 & 6 & 0 & -0.440261 & 5.206946 & 0.194132 \\
\hline 53 & 6 & 0 & -0.741855 & 3.859543 & 0.370292 \\
\hline 54 & 6 & 0 & -0.928979 & 1.511295 & -0.466649 \\
\hline
\end{tabular}




\begin{tabular}{|c|c|c|c|c|c|}
\hline 55 & 1 & 0 & -1.816521 & 0.113537 & 2.279773 \\
\hline 56 & 1 & 0 & -0.784016 & -0.734048 & 0.643452 \\
\hline 57 & 1 & 0 & -2.016826 & -0.805295 & -1.505224 \\
\hline 58 & 1 & 0 & -2.745117 & 2.560722 & -2.223753 \\
\hline 59 & 1 & 0 & -1.909907 & 1.116910 & -2.814491 \\
\hline 60 & 1 & 0 & -3.932714 & -0.020552 & -3.214401 \\
\hline 61 & 1 & 0 & -4.669593 & 1.581323 & -3.122580 \\
\hline 62 & 1 & 0 & -3.053292 & 2.881492 & 0.141884 \\
\hline 63 & 1 & 0 & -3.116163 & 1.430495 & 1.161967 \\
\hline 64 & 1 & 0 & -5.094664 & 2.503804 & -0.854145 \\
\hline 65 & 1 & 0 & -6.770686 & 1.743514 & 0.618383 \\
\hline 66 & 1 & 0 & -6.294192 & 0.756946 & 2.758127 \\
\hline 67 & 1 & 0 & -4.516398 & 0.501158 & 2.311708 \\
\hline 68 & 1 & 0 & -5.832645 & 0.133635 & -1.493511 \\
\hline 69 & 1 & 0 & -4.173452 & -1.659335 & -1.234154 \\
\hline 70 & 1 & 0 & -4.382555 & -0.924857 & 0.360162 \\
\hline 71 & 1 & 0 & 0.175565 & -1.590548 & -1.036095 \\
\hline 72 & 1 & 0 & 1.484788 & -2.917565 & -2.603445 \\
\hline 73 & 1 & 0 & 1.008672 & -5.345381 & -2.921445 \\
\hline 74 & 1 & 0 & -0.788327 & -6.434786 & -1.578544 \\
\hline 75 & 1 & 0 & -3.705541 & -5.178882 & 1.623781 \\
\hline 76 & 1 & 0 & -3.434362 & -2.712342 & 1.998983 \\
\hline 77 & 1 & 0 & -0.016128 & 2.734552 & -2.755462 \\
\hline 78 & 1 & 0 & 0.509382 & 5.132782 & -3.067447 \\
\hline 79 & 1 & 0 & 0.237104 & 6.718806 & -1.180689 \\
\hline 80 & 1 & 0 & -0.531272 & 5.893402 & 1.029934 \\
\hline 81 & 1 & 0 & -1.037798 & 3.470652 & 1.345000 \\
\hline 82 & 1 & 0 & -0.300096 & 0.860197 & -1.082143 \\
\hline 83 & 1 & 0 & -0.781165 & 1.285819 & 0.589319 \\
\hline 84 & 8 & 0 & 6.138528 & -2.610265 & -0.259821 \\
\hline 85 & 1 & 0 & 6.024336 & -3.485657 & 0.148736 \\
\hline
\end{tabular}




\section{NMR spectra and HPLC chromatograms}
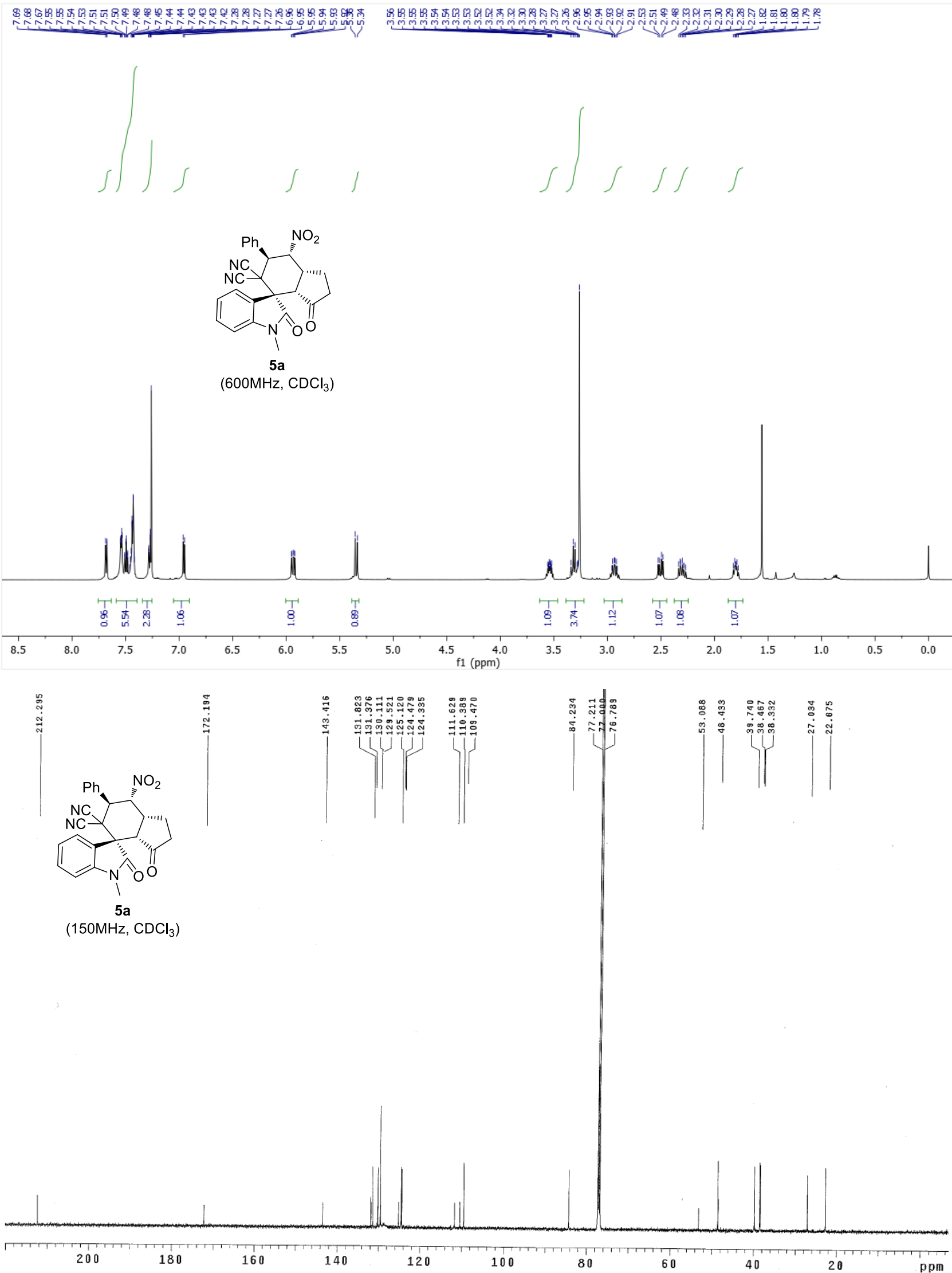


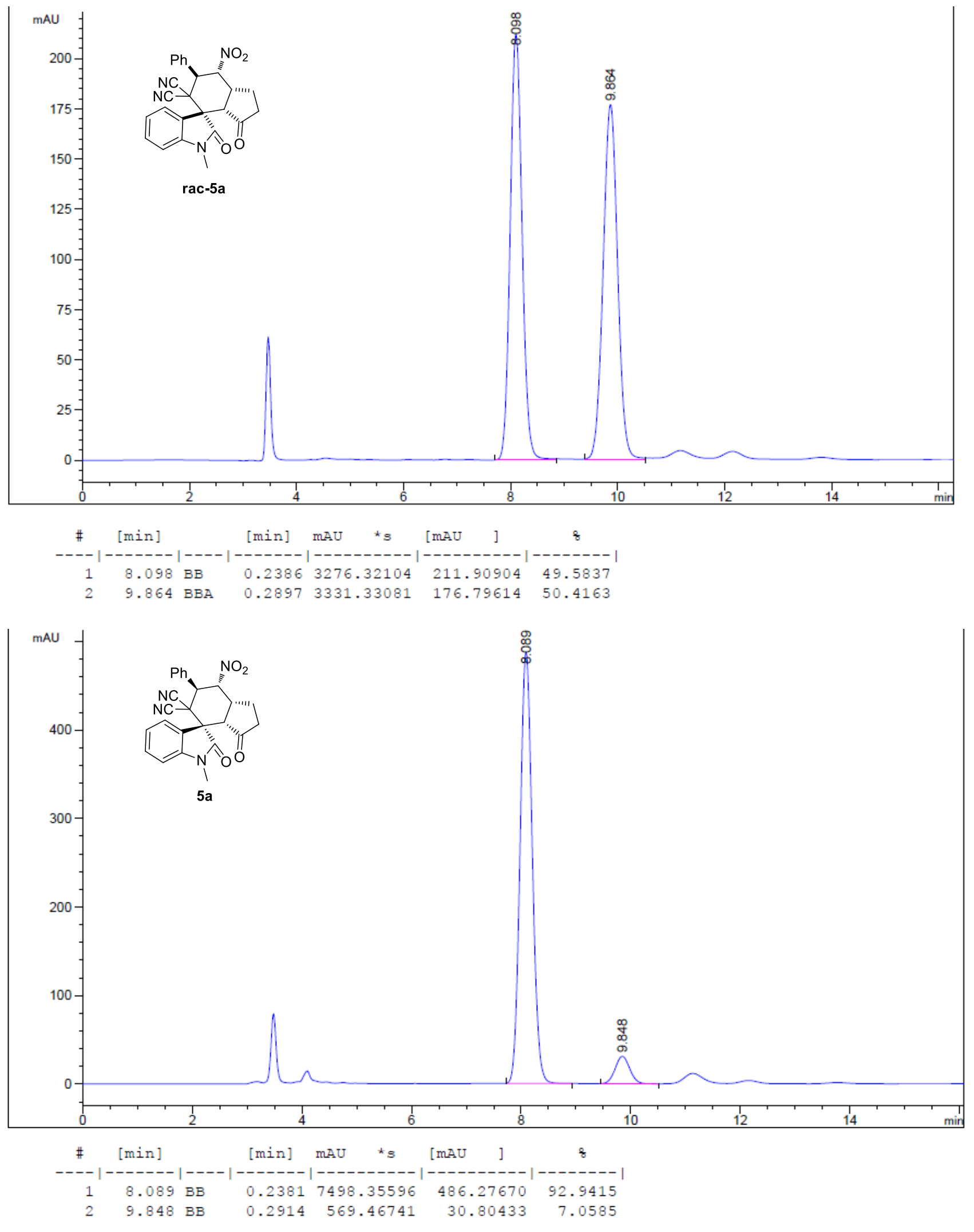



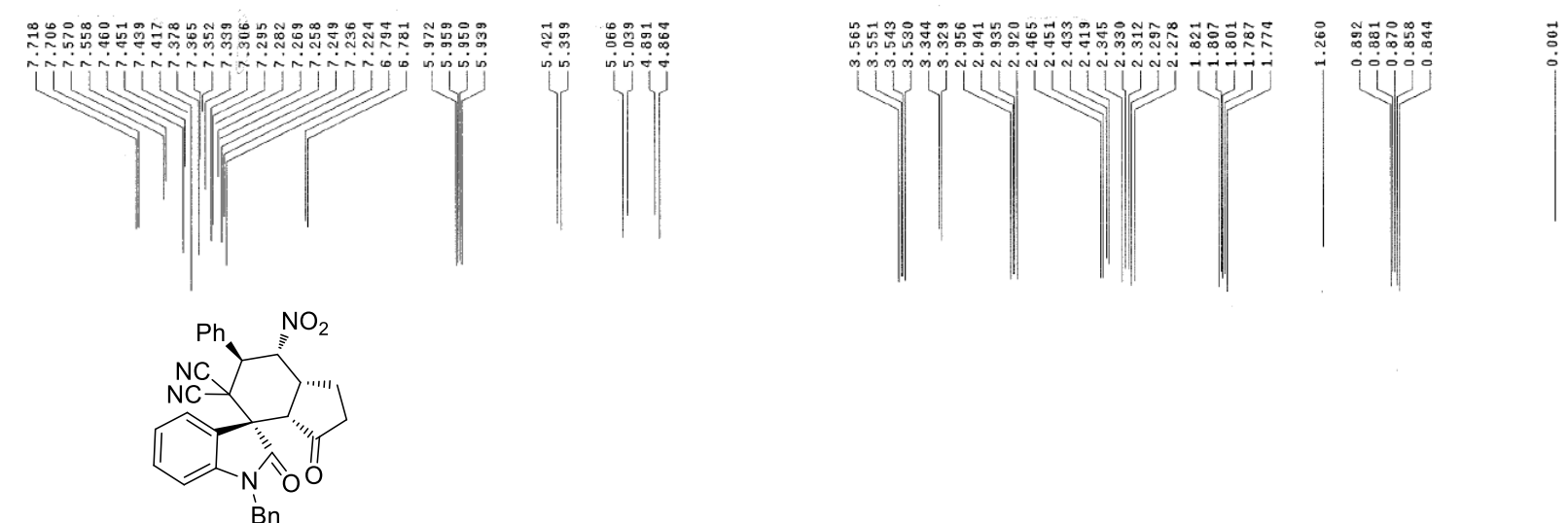

(600MHz, $\mathrm{CDCl}_{3}$ )
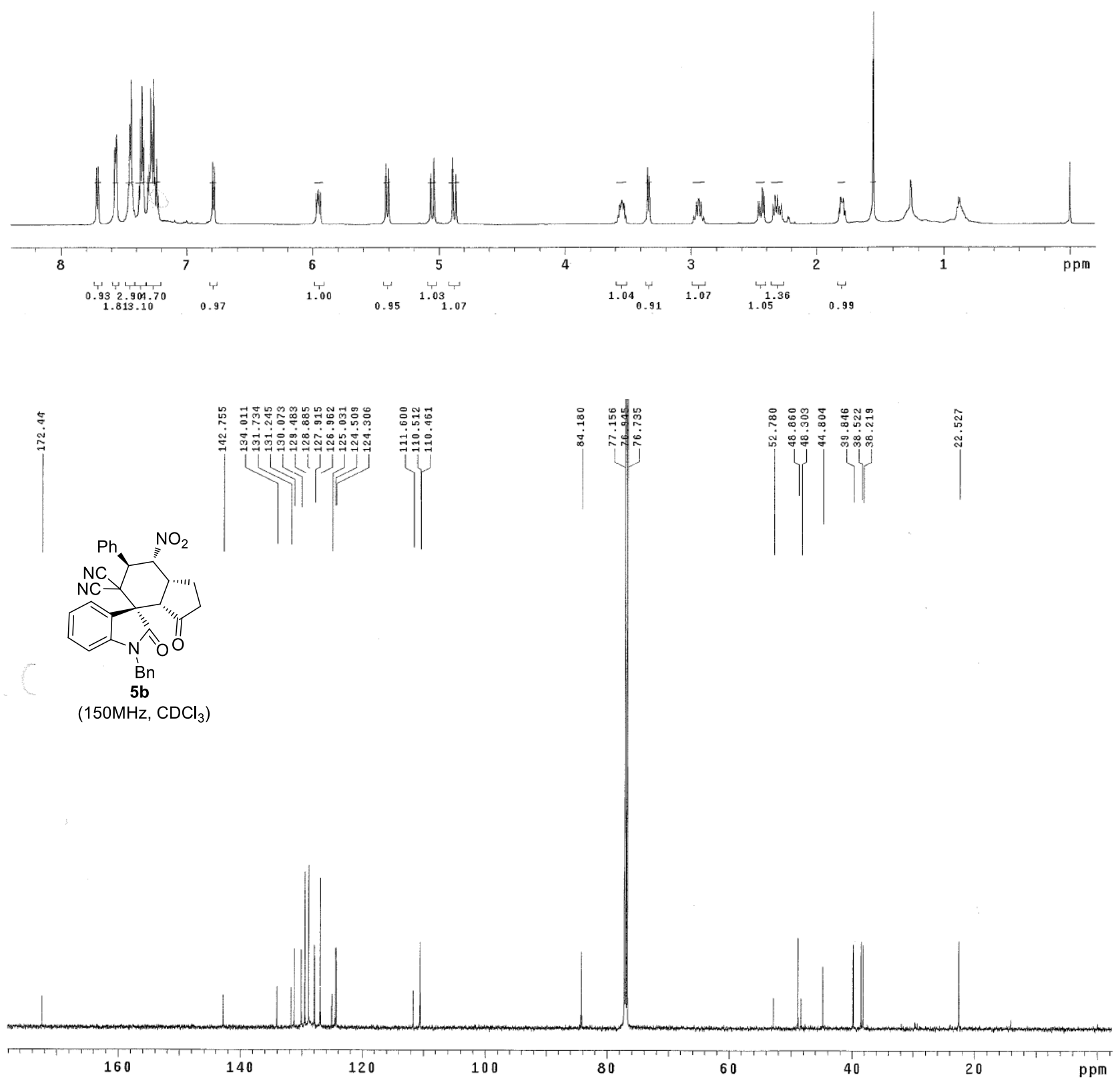


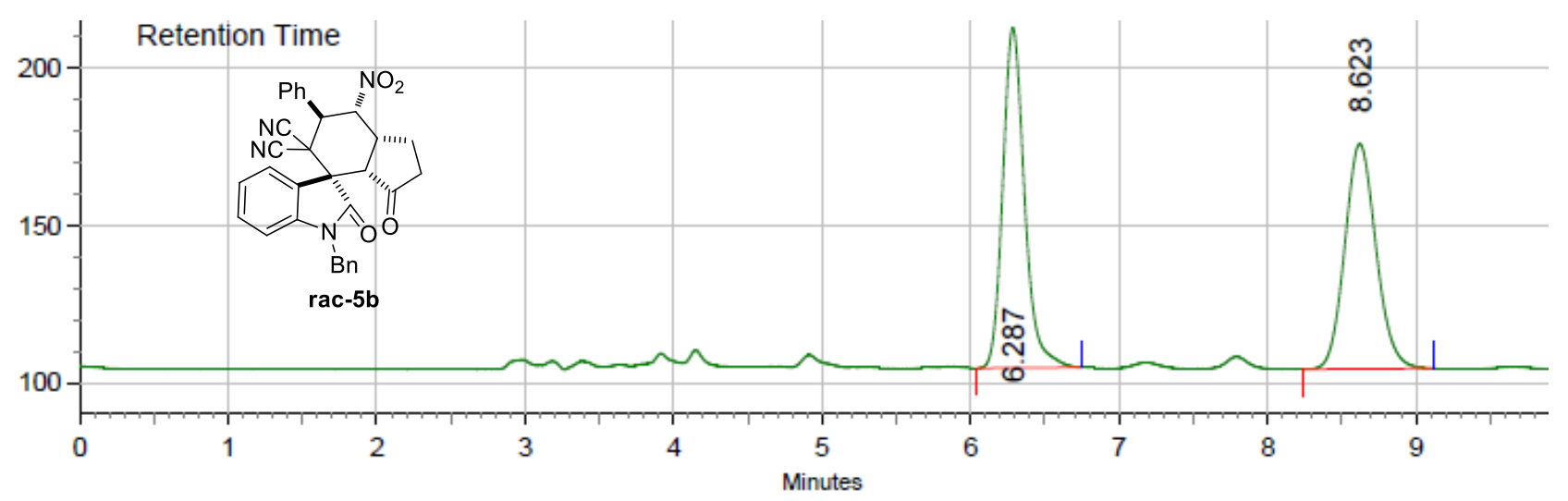

\begin{tabular}{|c|c|c|c|c|c|}
\hline Peak & RT (min) & Height & \% Height & Area & \% Area \\
\hline 1 & 6.287 & 1809114 & 60.18 & 18437039 & 51.25 \\
\hline 2 & 8.623 & 1197170 & 39.82 & 17536246 & 48.75 \\
\hline
\end{tabular}

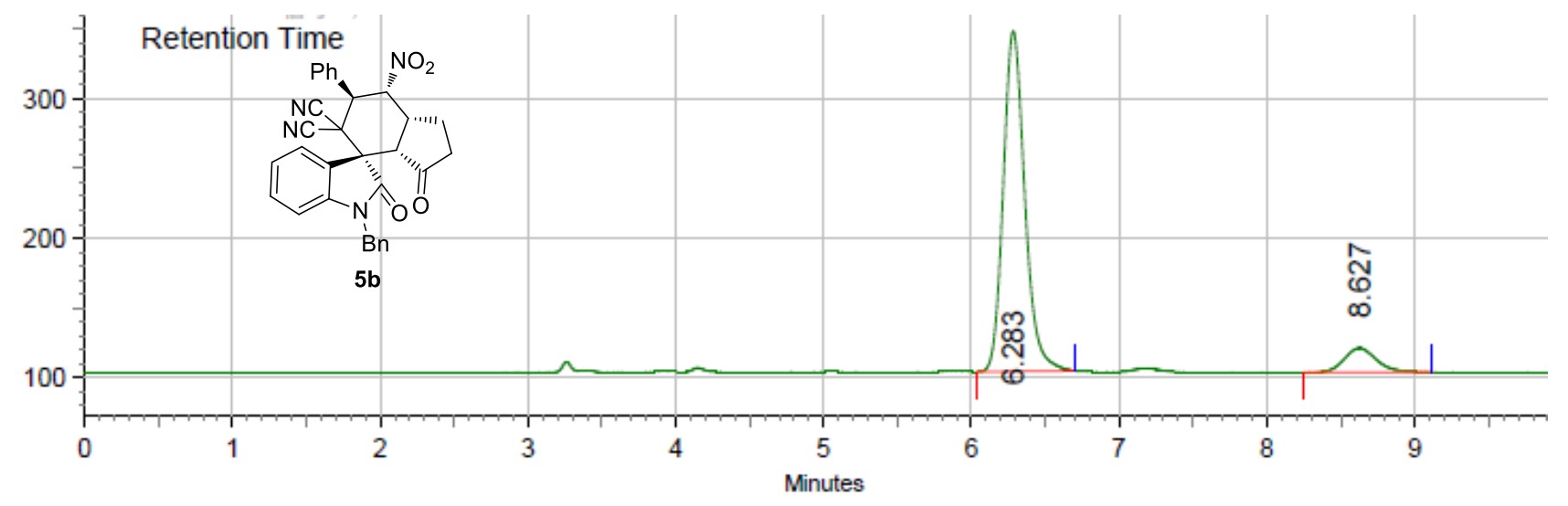

\begin{tabular}{|c|c|c|c|c|c|}
\hline Peak & RT (min) & Height & \% Height & Area & \% Area \\
\hline 1 & 6.283 & 4083472 & 93.42 & 41391473 & 90.52 \\
\hline 2 & 8.627 & 287437 & 6.58 & 4332692 & 9.48 \\
\hline
\end{tabular}




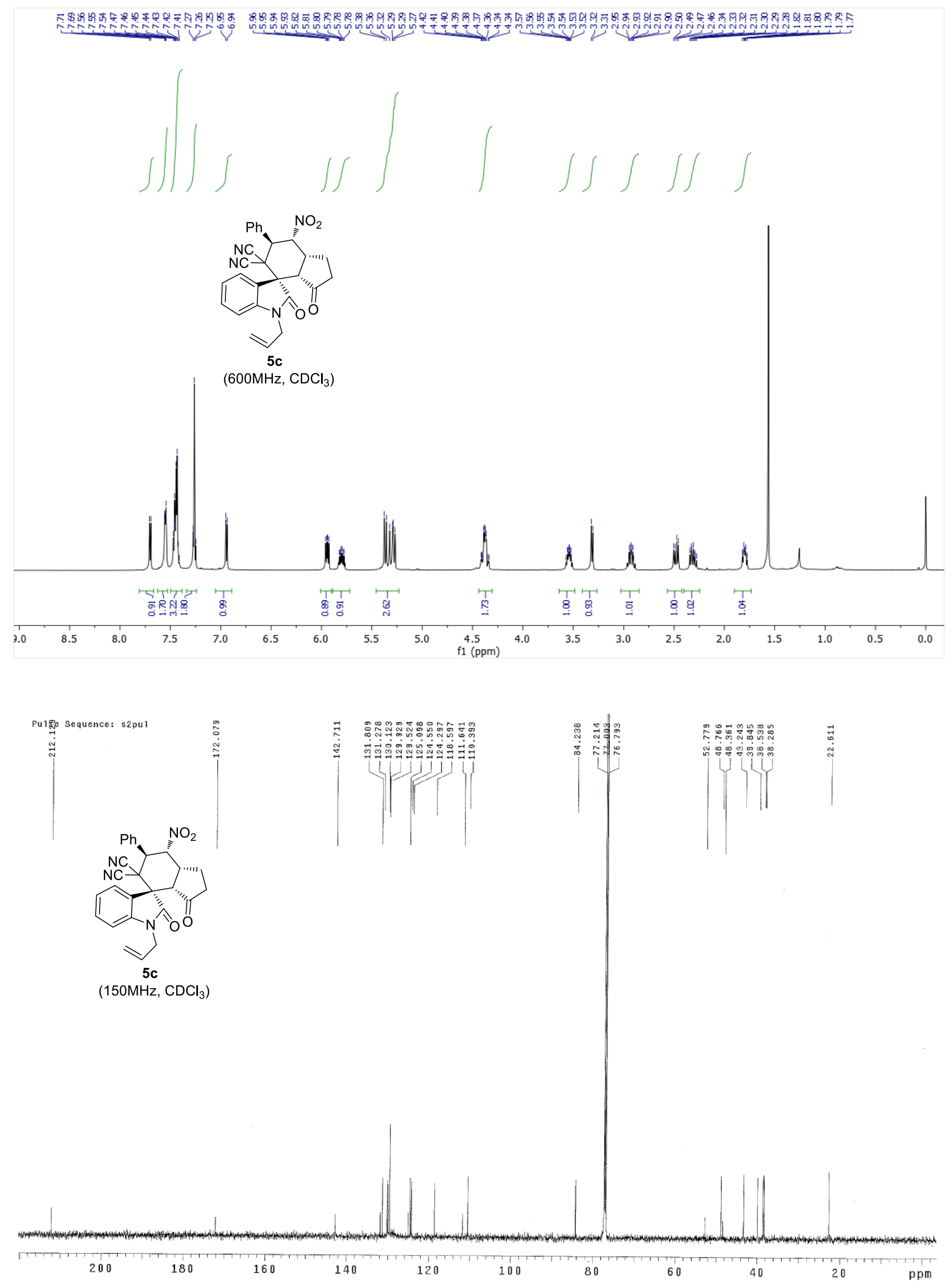



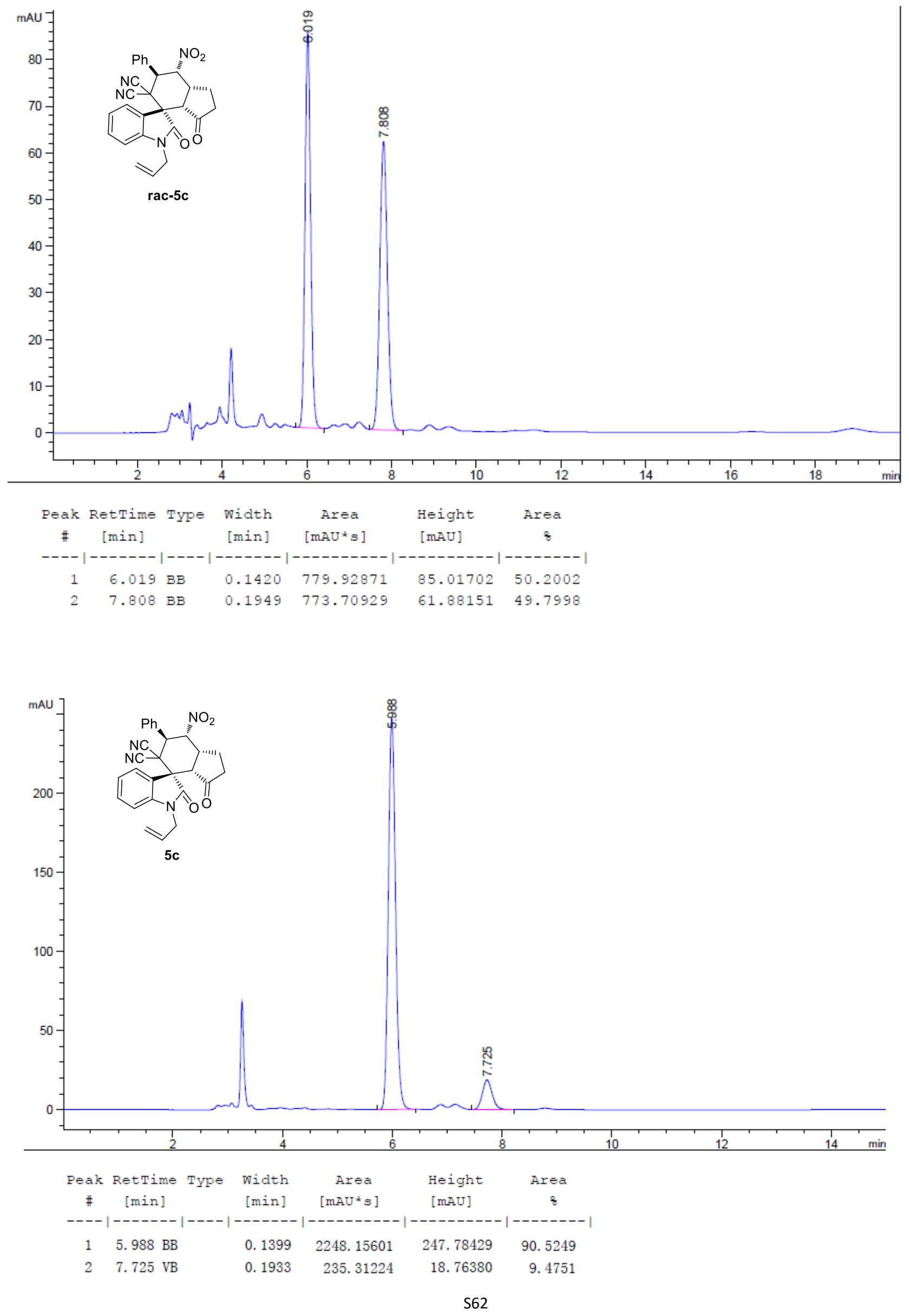

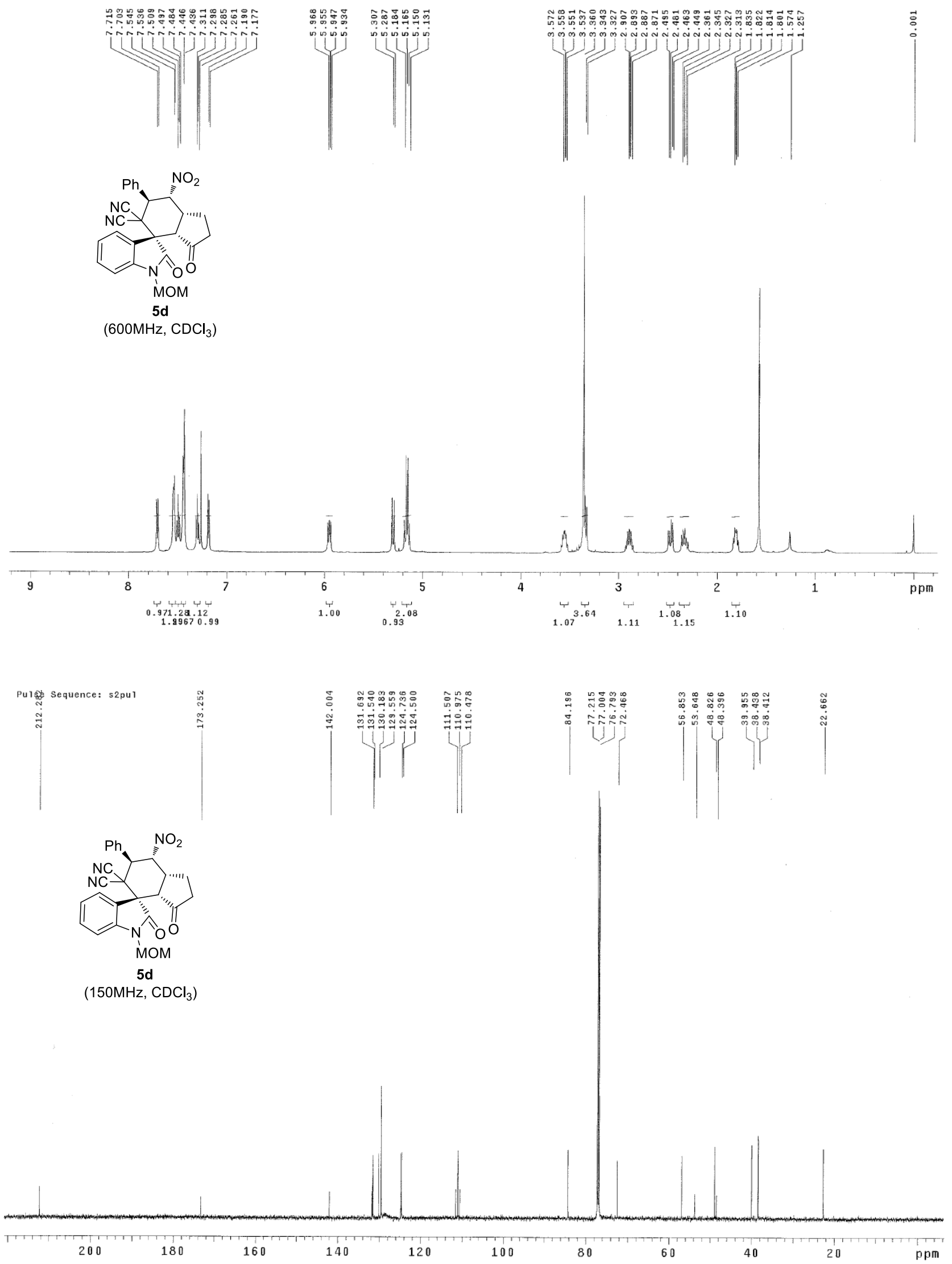

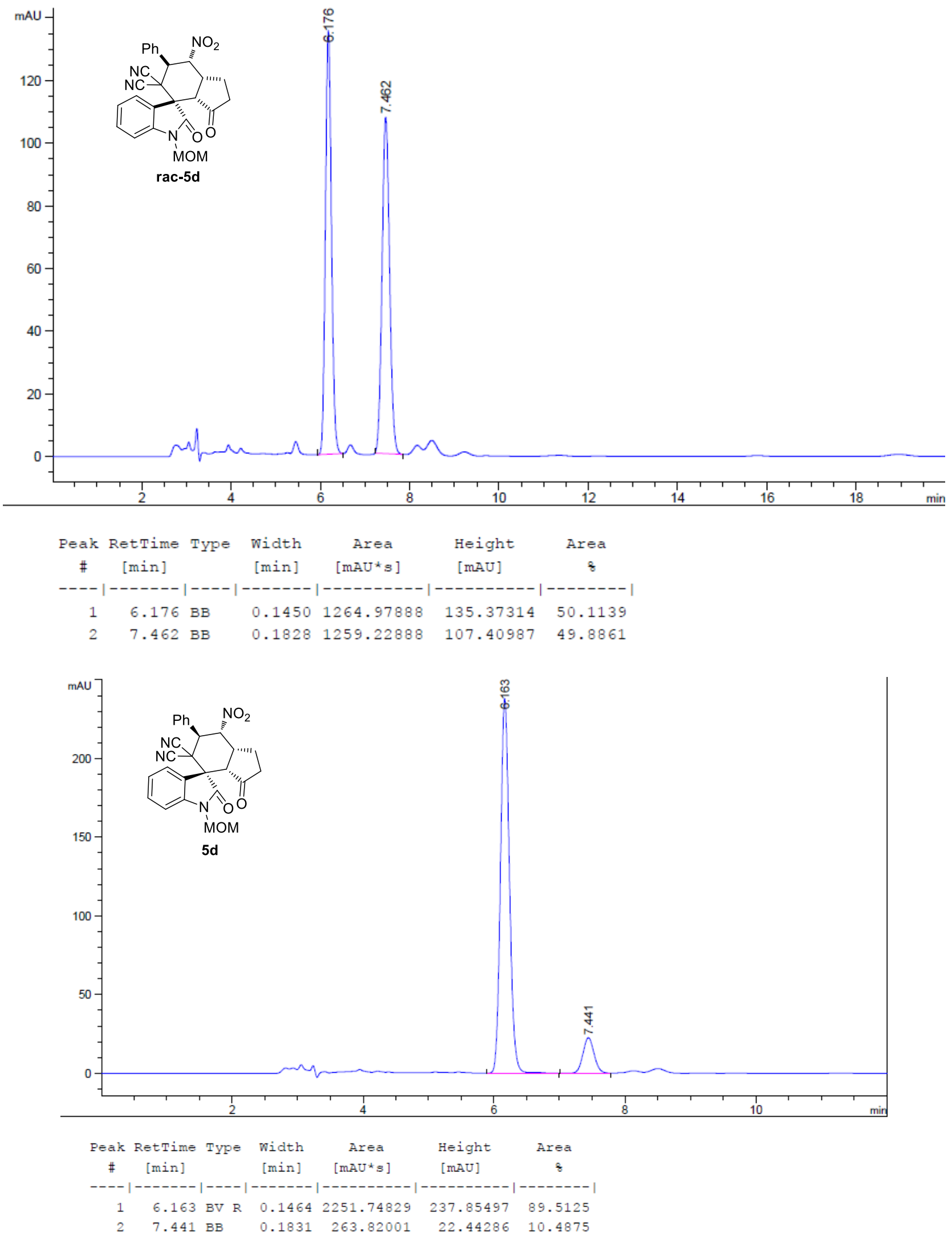

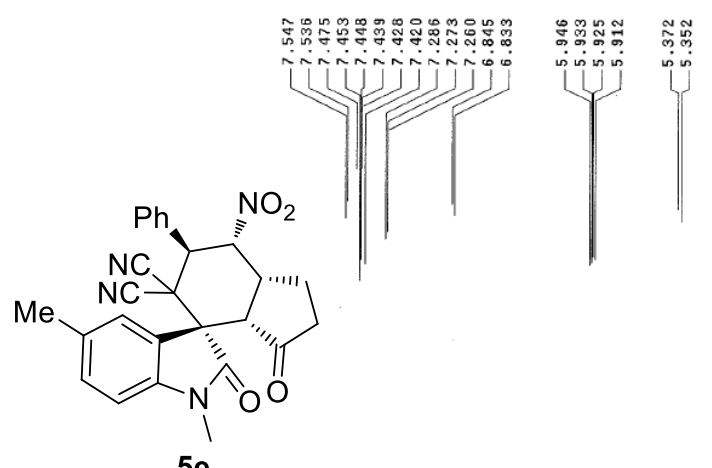

$\left(600 \mathrm{MHz}, \mathrm{CDCl}_{3}\right)$
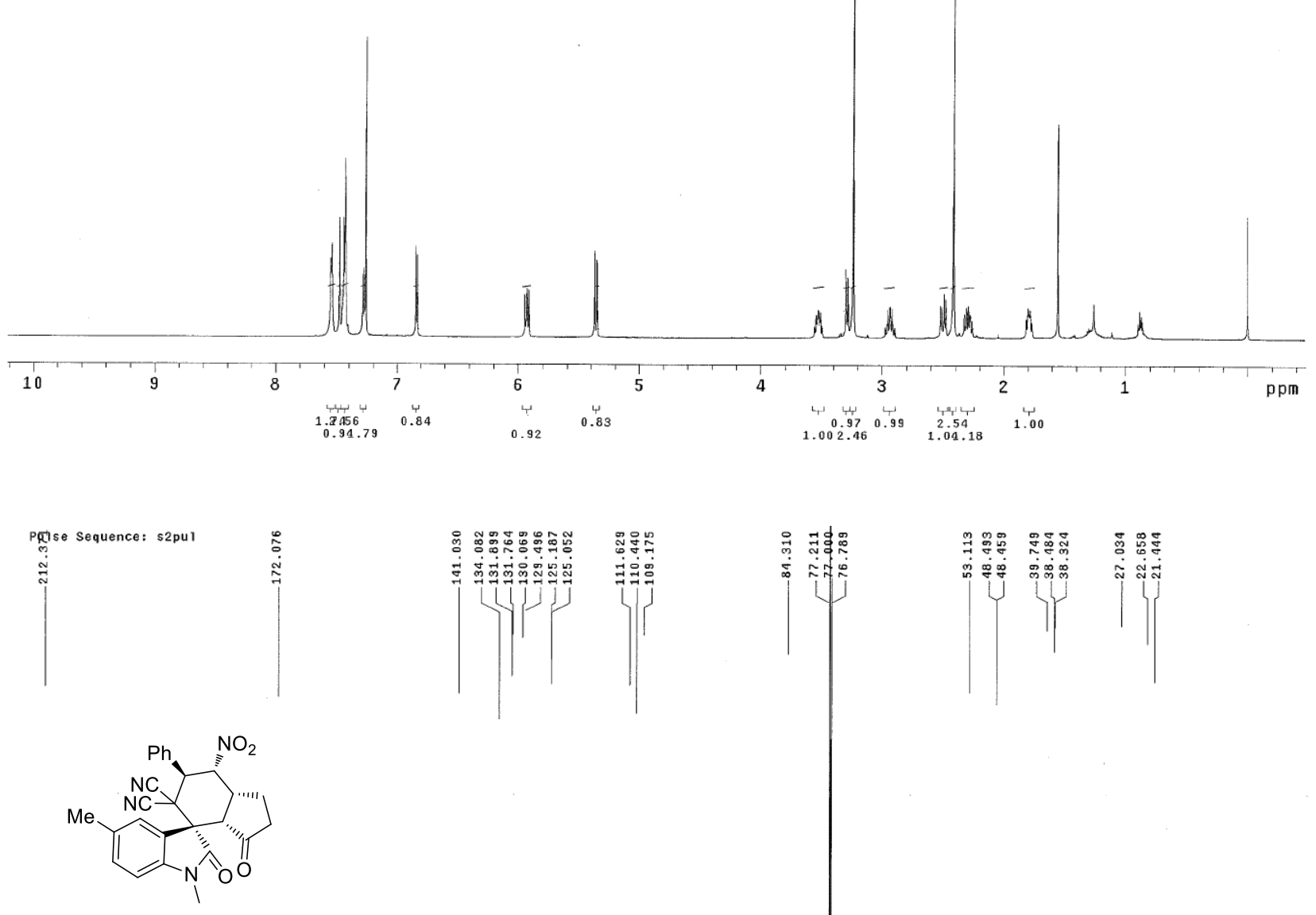

$\left(150 \mathrm{MHz}, \mathrm{CDCl}_{3}\right)$

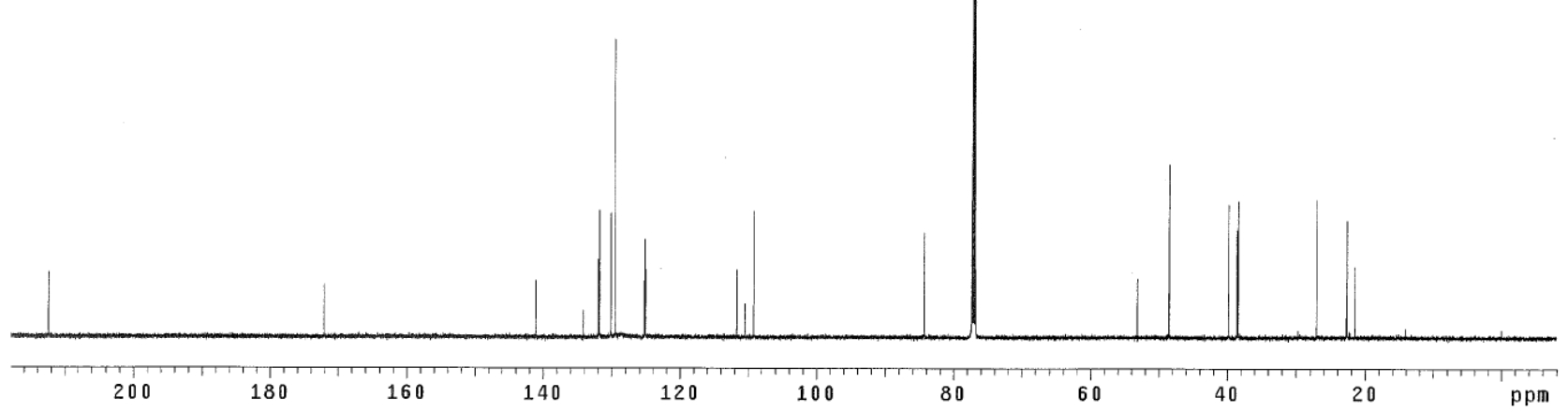




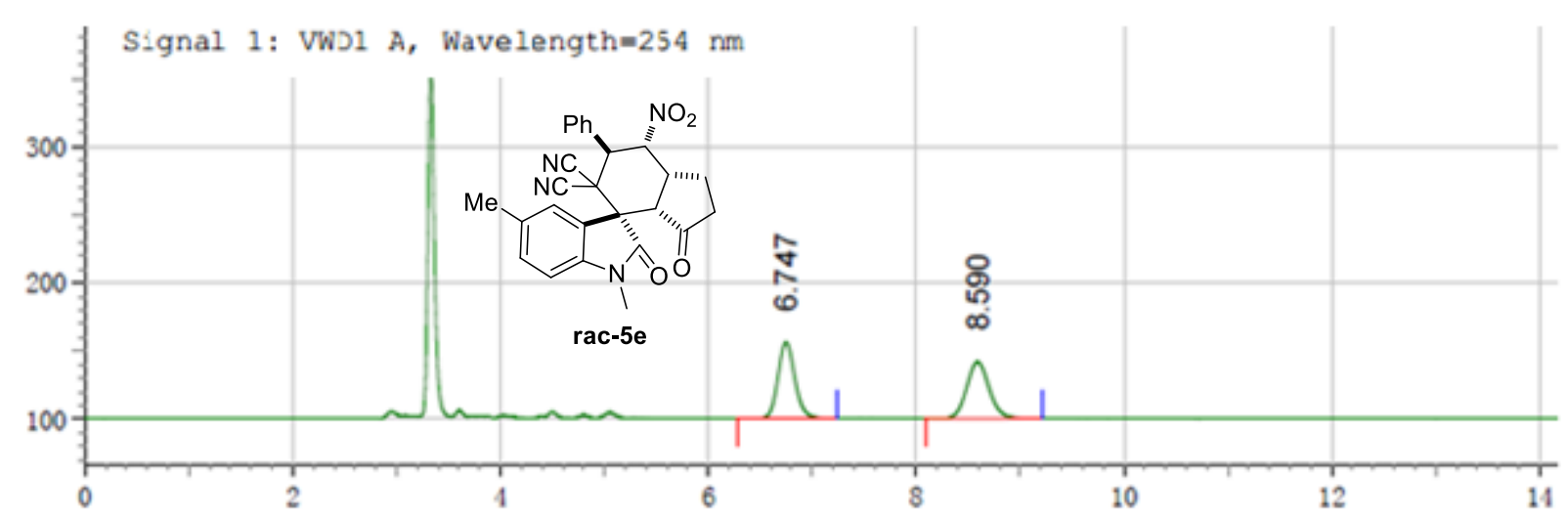

\begin{tabular}{|c|c|c|c|c|c|}
\hline Peak & RT (min) & Height & \% Height & Area & \% Area \\
\hline 1 & 6.747 & 939051 & 57.45 & 10599081 & 50.29 \\
\hline 2 & 8.590 & 695641 & 42.55 & 10476522 & 49.71 \\
\hline
\end{tabular}

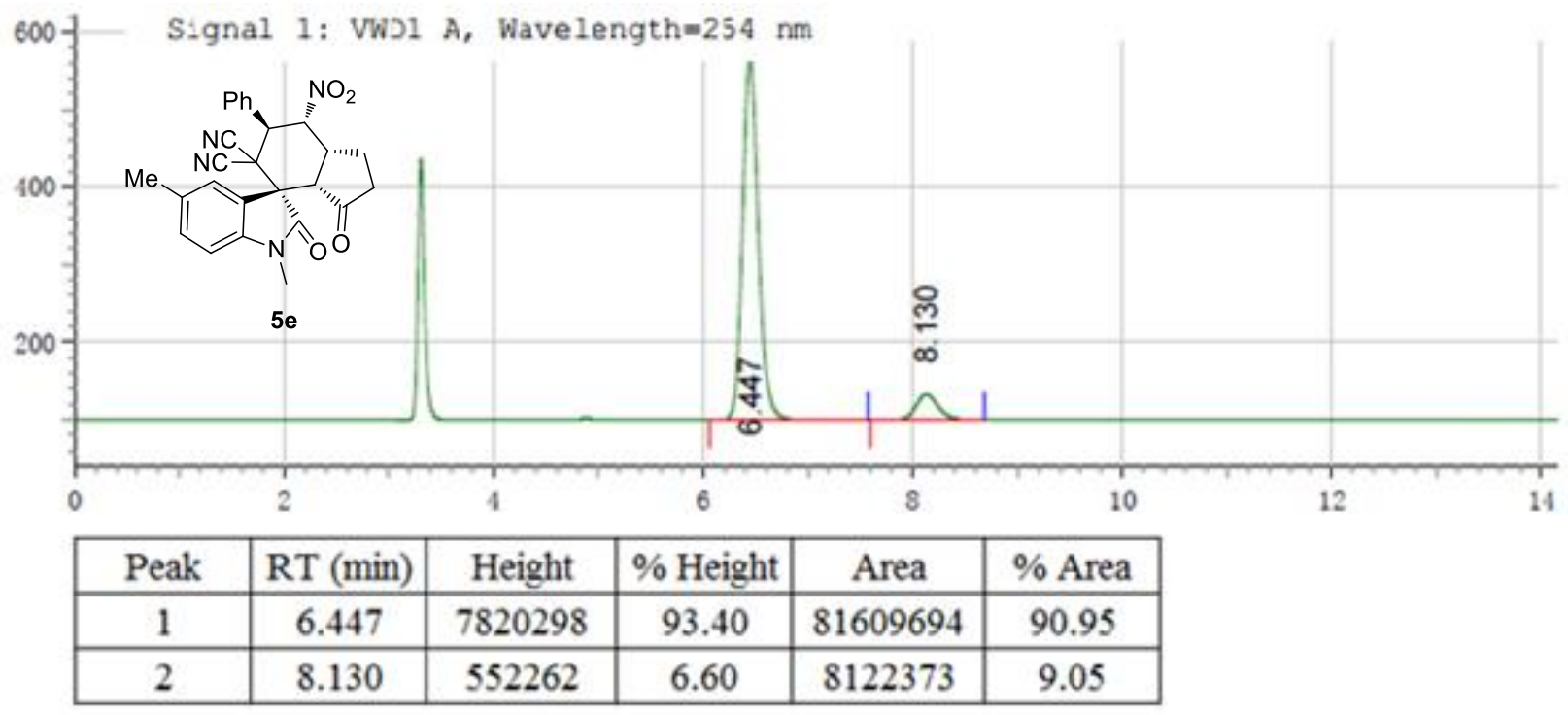




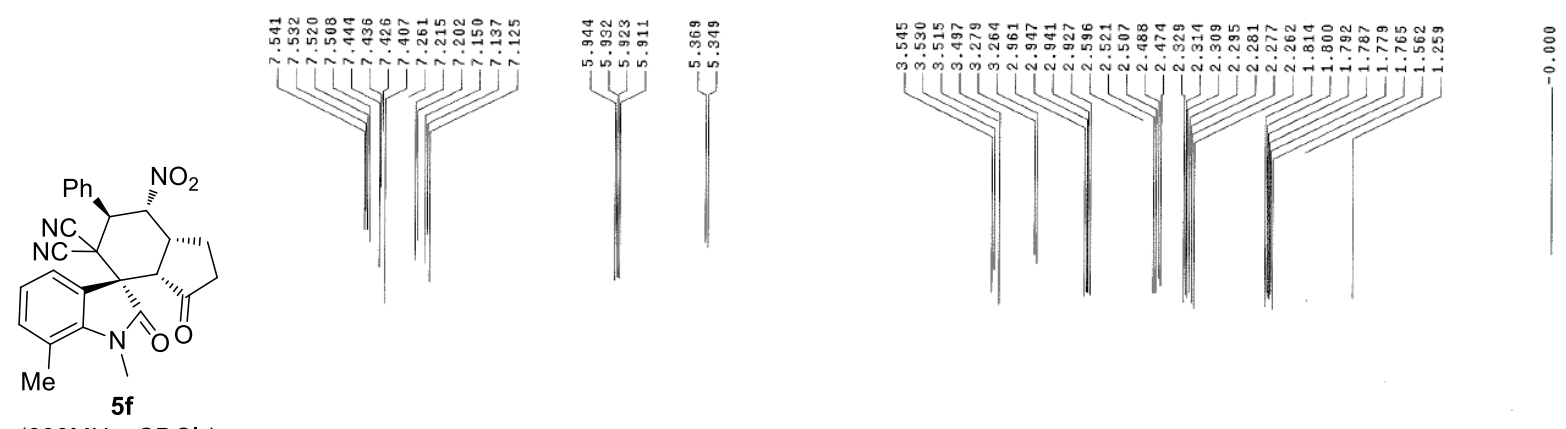

$\left(600 \mathrm{MHz}, \mathrm{CDCl}_{3}\right)$
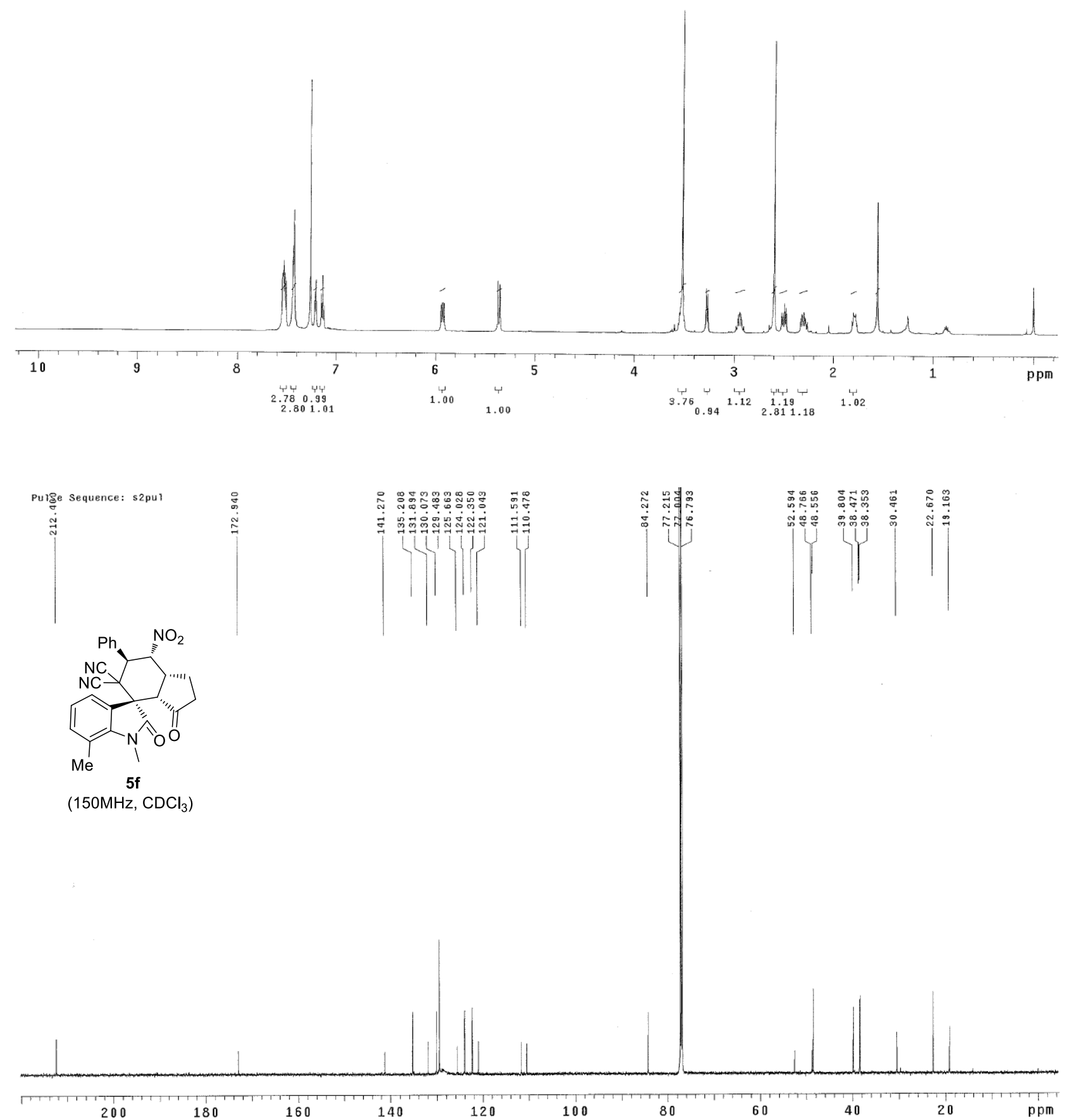


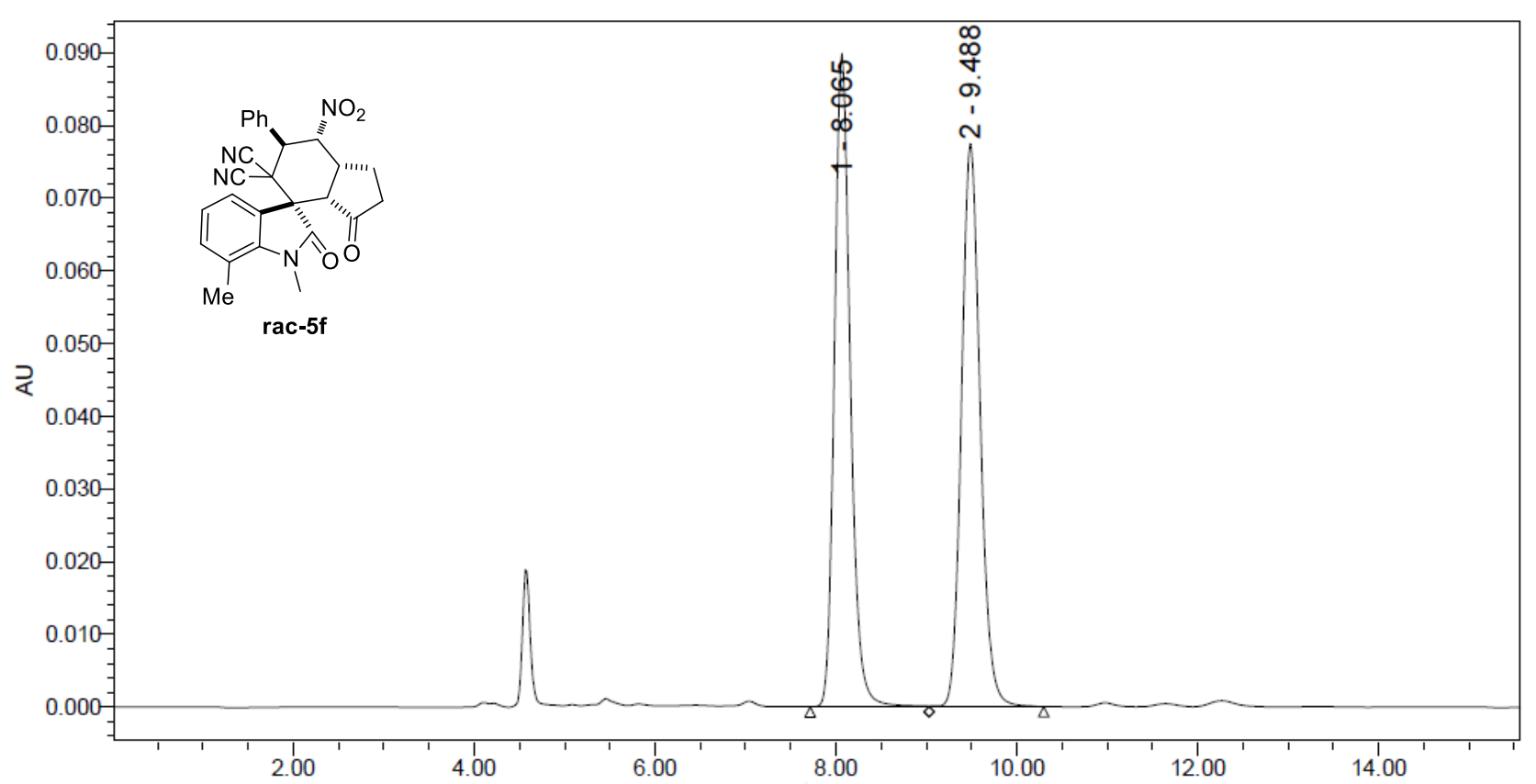

\begin{tabular}{|c|c|c|c|c|c|}
\hline Peak & RT (min) & Height & \% Height & Area & \% Area \\
\hline 1 & 8.065 & 89818 & 53.68 & 1094525 & 49.69 \\
\hline 2 & 9.488 & 77502 & 46.32 & 1108114 & 50.31 \\
\hline
\end{tabular}

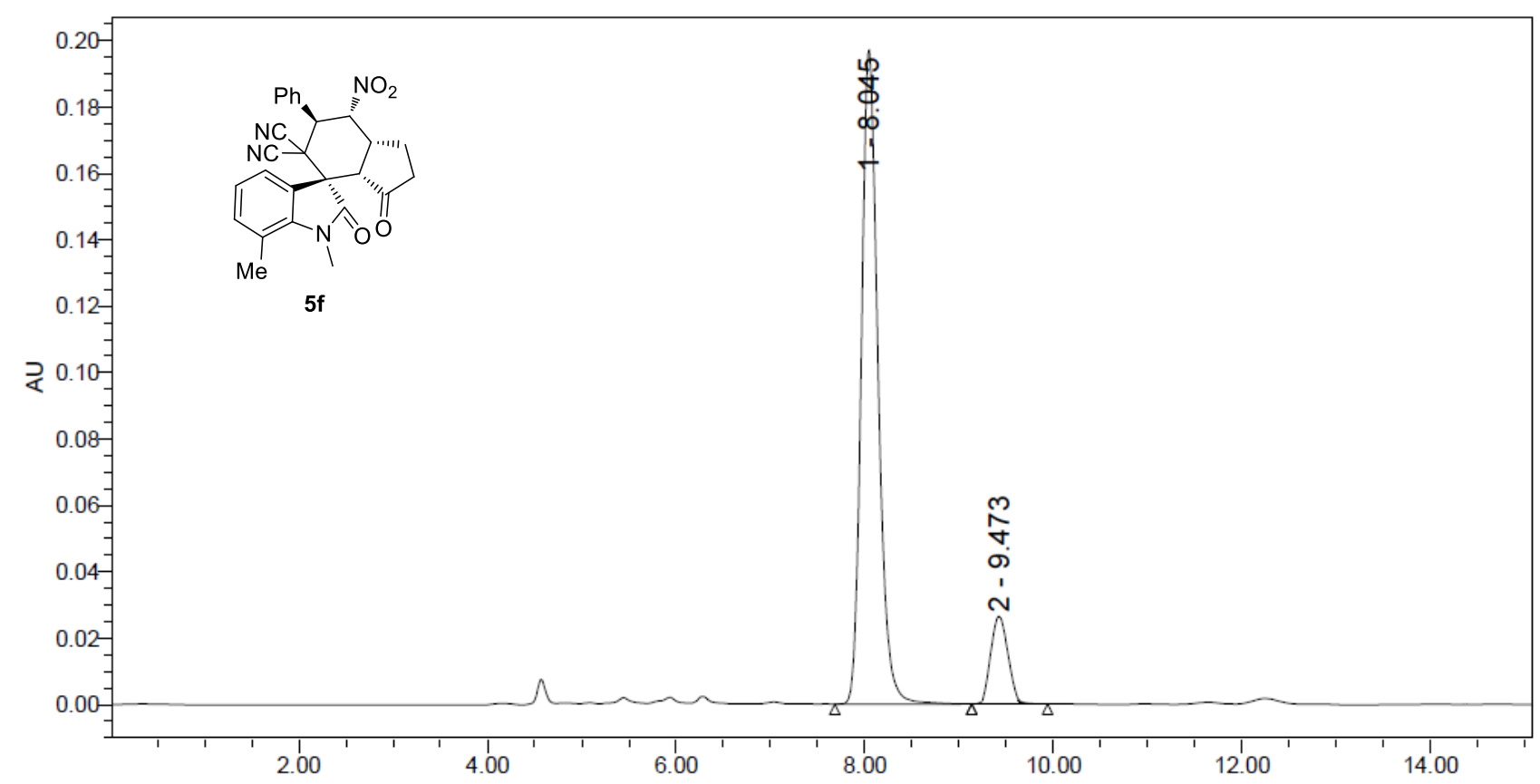

\begin{tabular}{|c|c|c|c|c|c|}
\hline Peak & RT (min) & Height & \% Height & Area & \% Area \\
\hline 1 & 8.045 & 197523 & 86.94 & 2518839 & 92.02 \\
\hline 2 & 9.473 & 29683 & 13.06 & 331093 & 7.98 \\
\hline
\end{tabular}




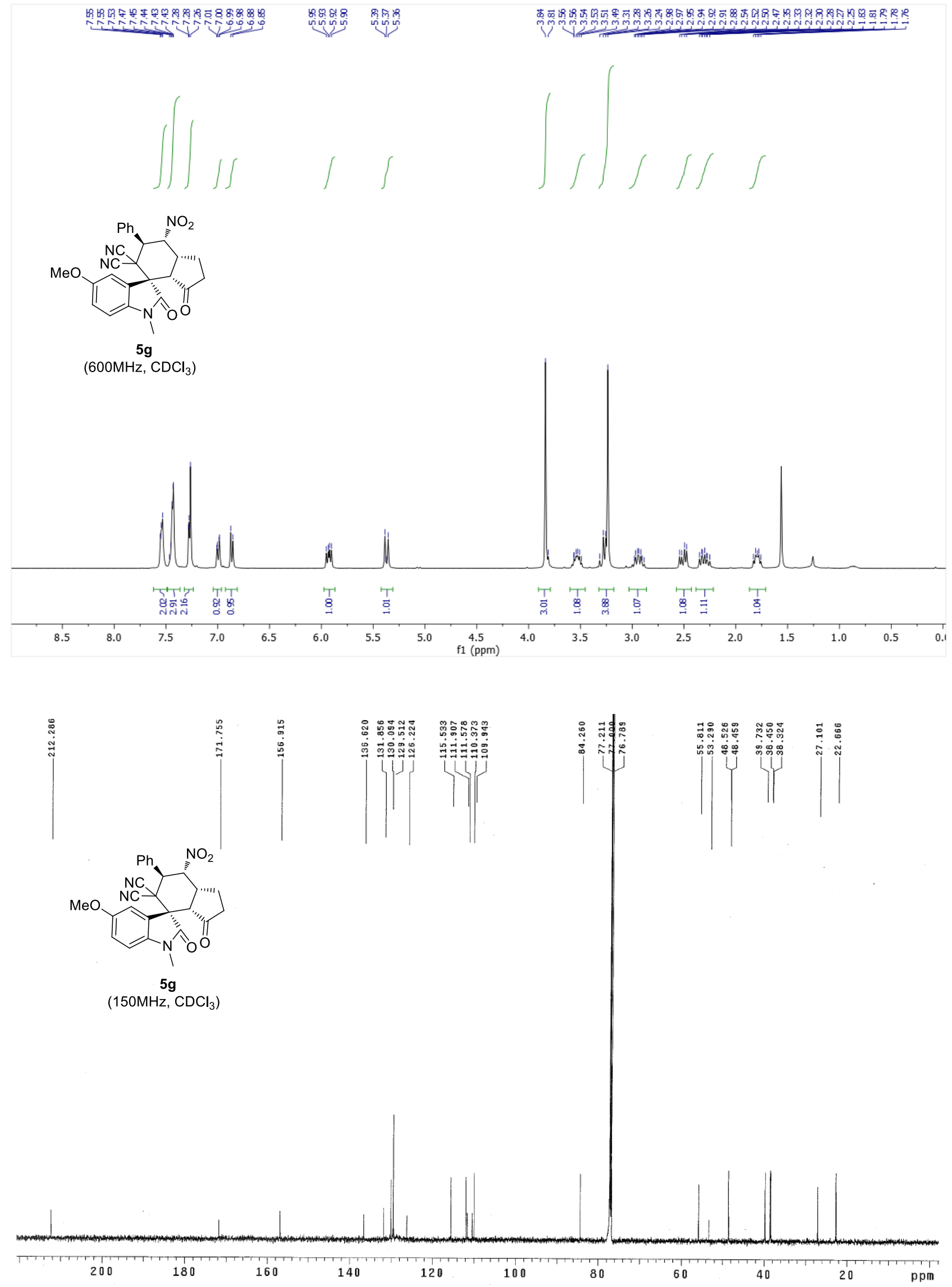




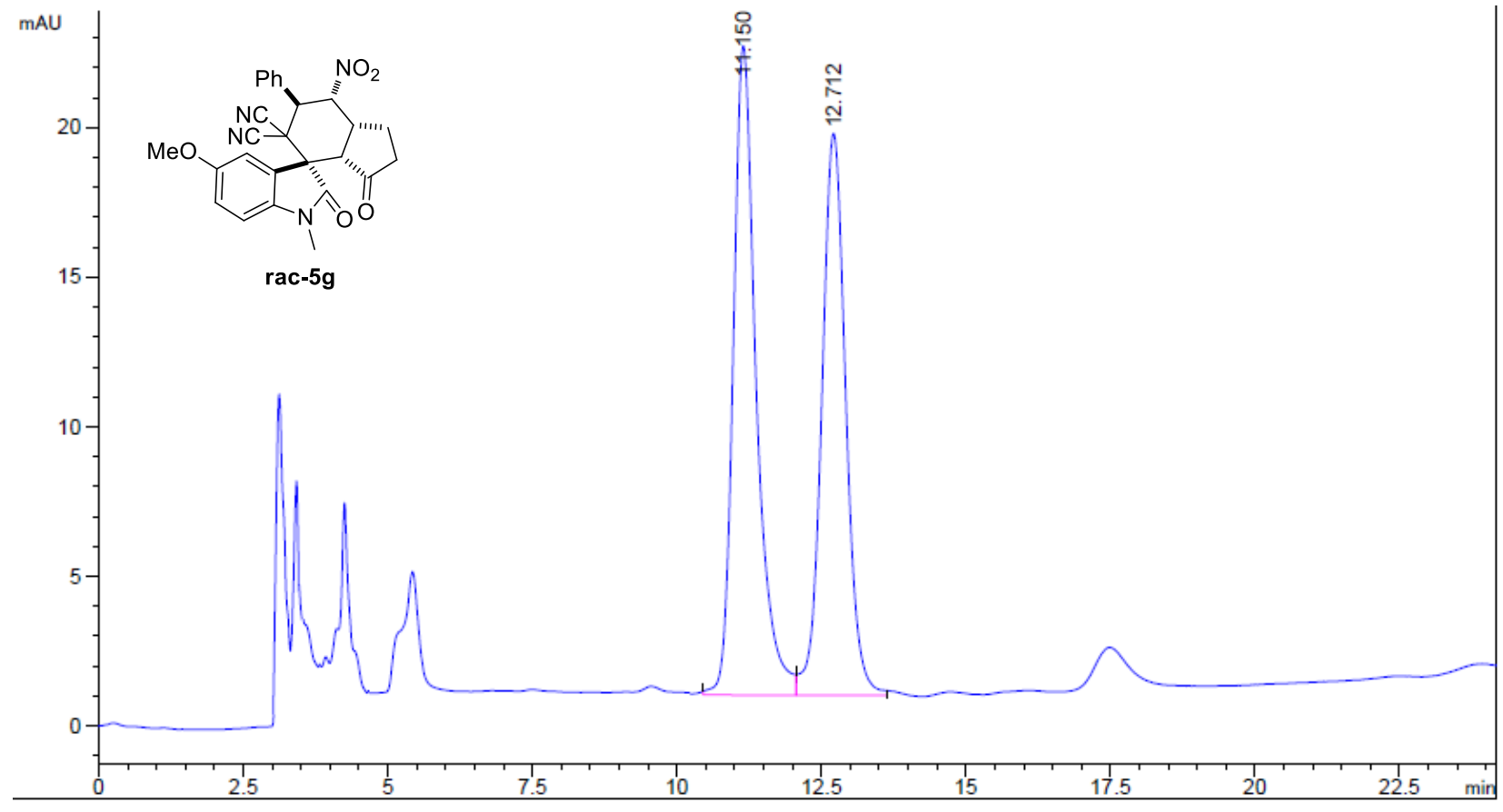

\begin{tabular}{|c|c|c|c|c|c|}
\hline Peak & RT $(\mathrm{min})$ & Height & \% Height & Area & \% Area \\
\hline 1 & 11.150 & 21.68 & 58.84 & 600.22 & 52.55 \\
\hline 2 & 12.712 & 18.77 & 41.16 & 541.93 & 47.45 \\
\hline
\end{tabular}

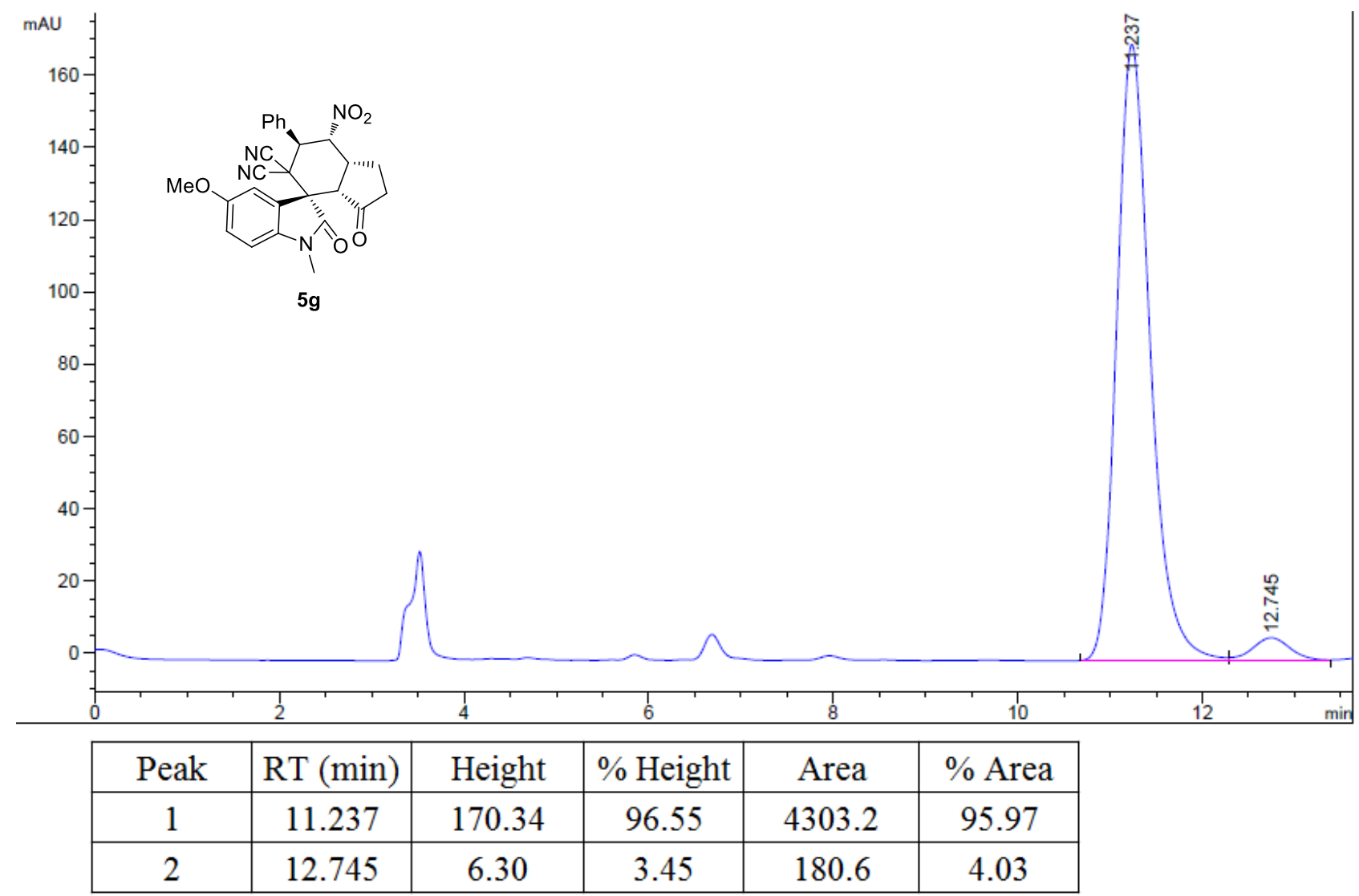



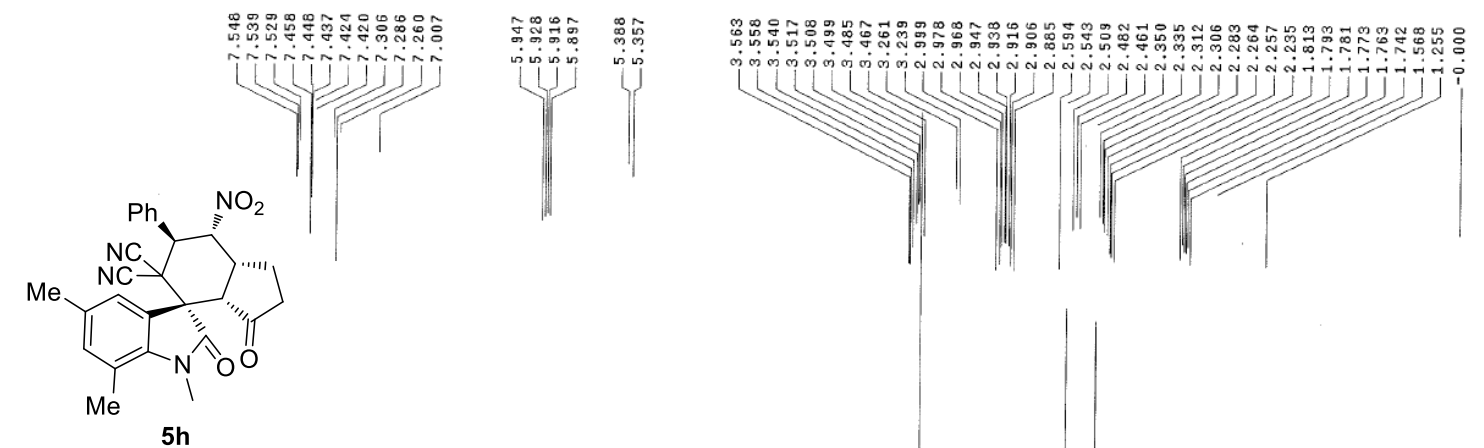

(400MHz, $\mathrm{CDCl}_{3}$ )
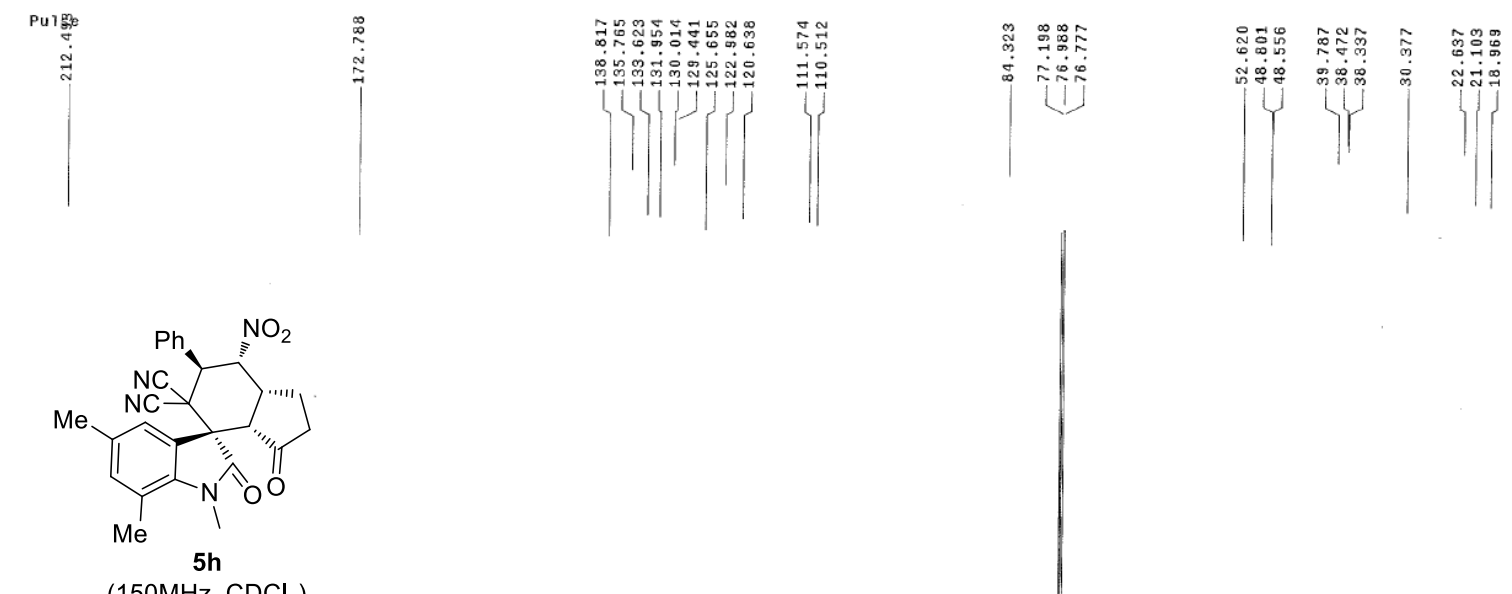

(150MHz, $\mathrm{CDCl}_{3}$ )

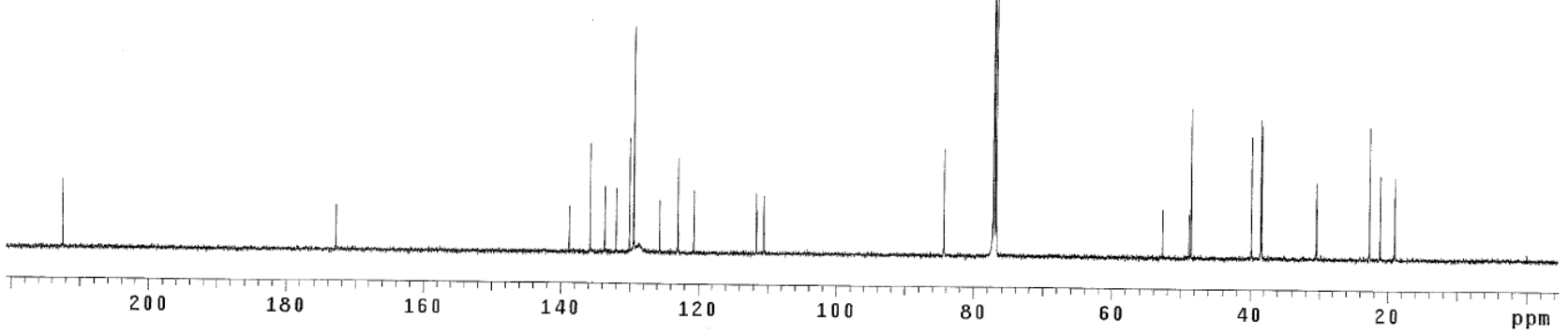



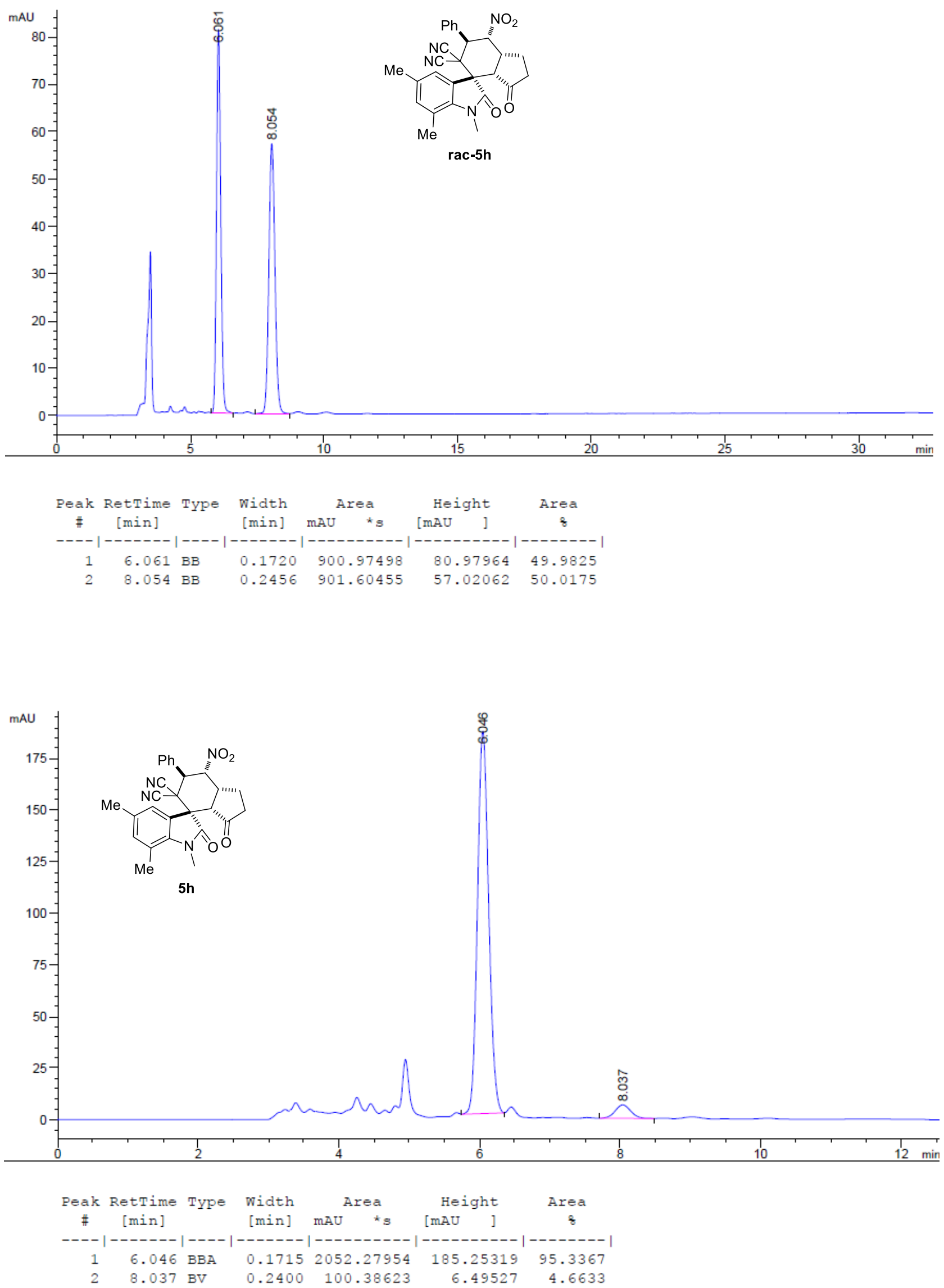

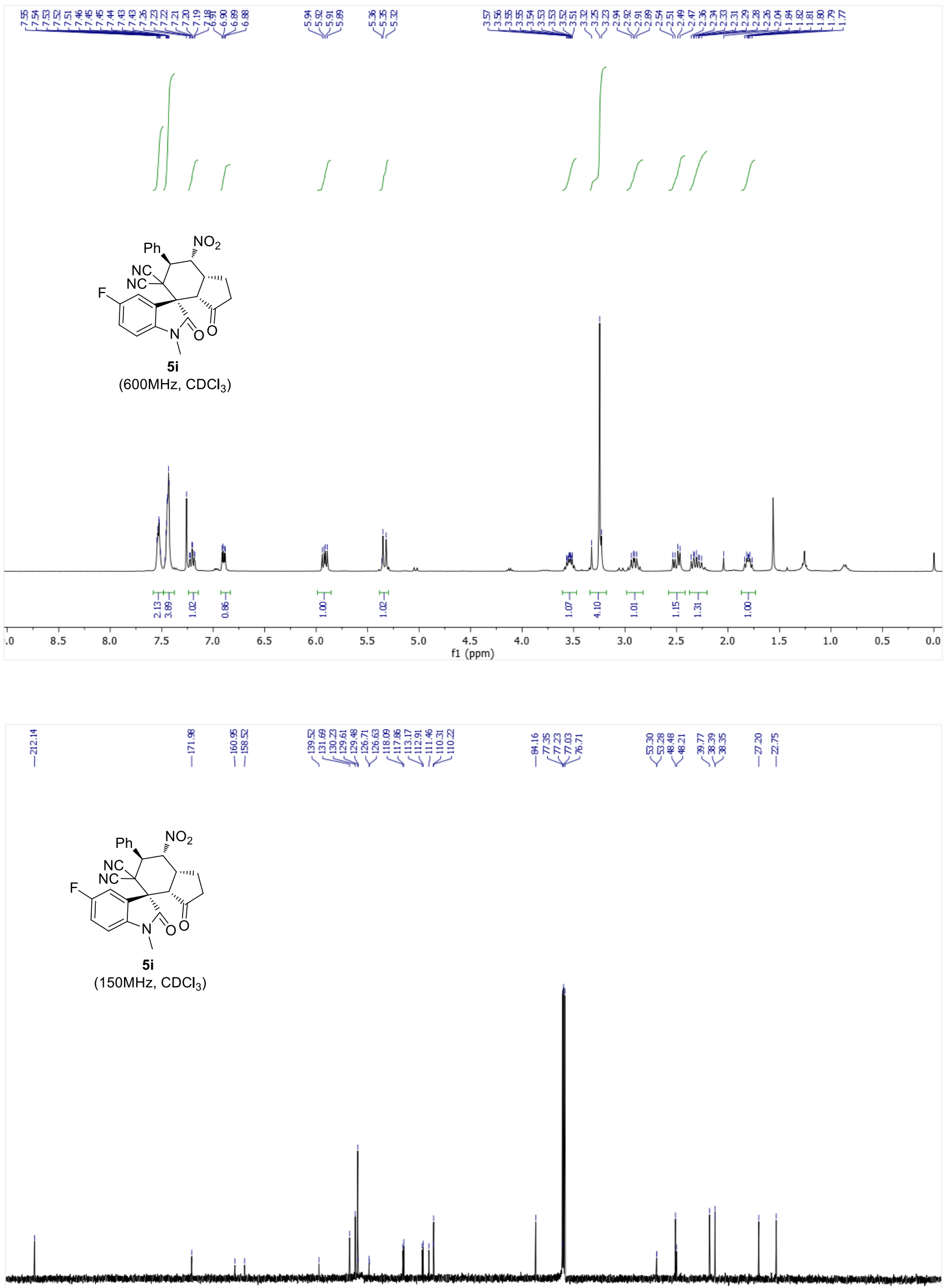

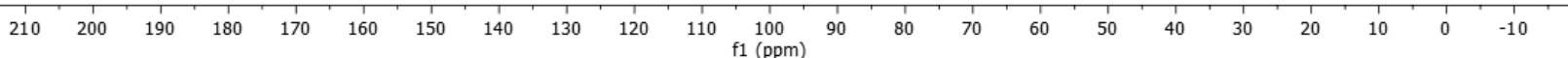




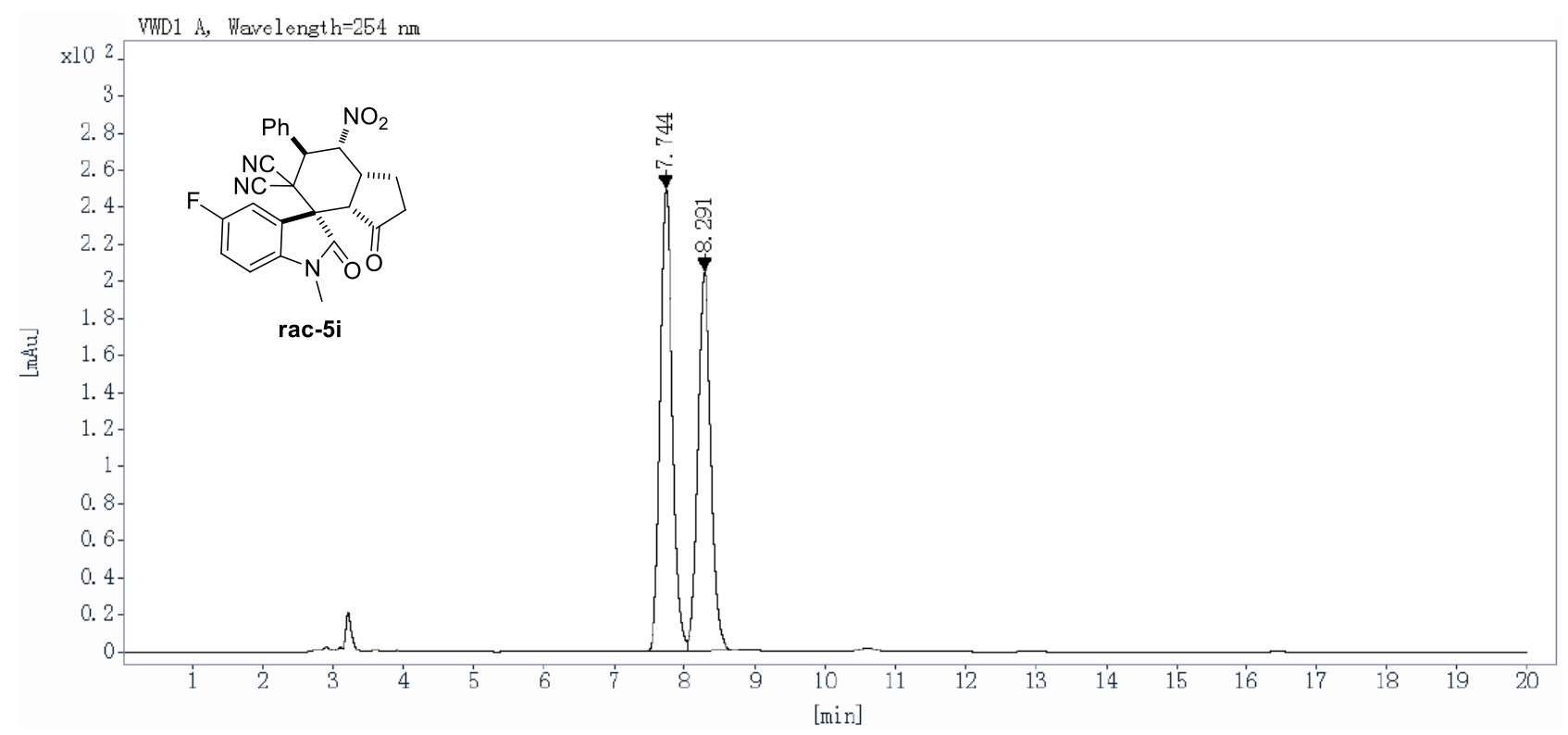

\begin{tabular}{cccccc}
$\begin{array}{c}\text { Ret Time } \\
\text { [min] }\end{array}$ & $\begin{array}{c}\text { Peak } \\
\text { Type }\end{array}$ & $\begin{array}{c}\text { Width } \\
{[\mathrm{min}]}\end{array}$ & $\begin{array}{c}\text { Height } \\
{[\mathrm{mAU}]}\end{array}$ & $\begin{array}{c}\text { Area } \\
{\left[\mathrm{mAU}^{*} \mathrm{~s}\right]}\end{array}$ & $\begin{array}{c}\text { Area } \\
{[\%]}\end{array}$ \\
\hline 7.744 & BV & 0.19 & 249.9336 & 3032.8333 & 53.2981 \\
8.291 & VB & 0.20 & 204.9978 & 2657.4839 & 46.7019
\end{tabular}

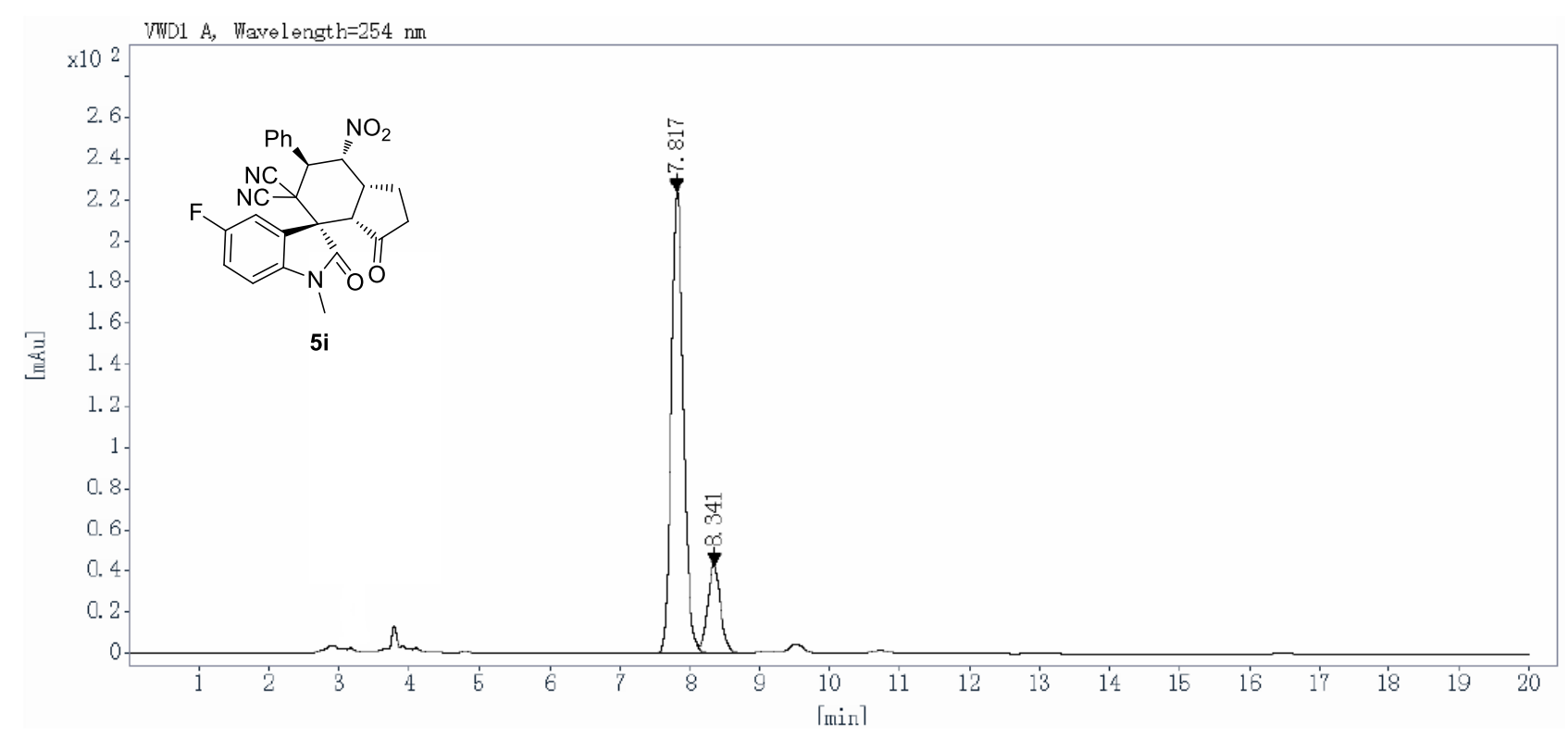

$\begin{array}{cccccc}\begin{array}{c}\text { Ret Time } \\ \text { [min] }\end{array} & \begin{array}{c}\text { Peak } \\ \text { Type }\end{array} & \begin{array}{c}\text { Width } \\ \text { [min] }\end{array} & \begin{array}{c}\text { Height } \\ {[\mathrm{mAU}]}\end{array} & \begin{array}{c}\text { Area } \\ {\left[\mathrm{mAU}^{*} \text { s] }\right.}\end{array} & \begin{array}{c}\text { Area } \\ {[\%]}\end{array} \\ 7.817 & \text { BV R } & 0.19 & 224.0534 & 2728.4934 & 83.0500 \\ 8.341 & \text { VB E } & 0.20 & 42.7743 & 556.8672 & 16.9500\end{array}$




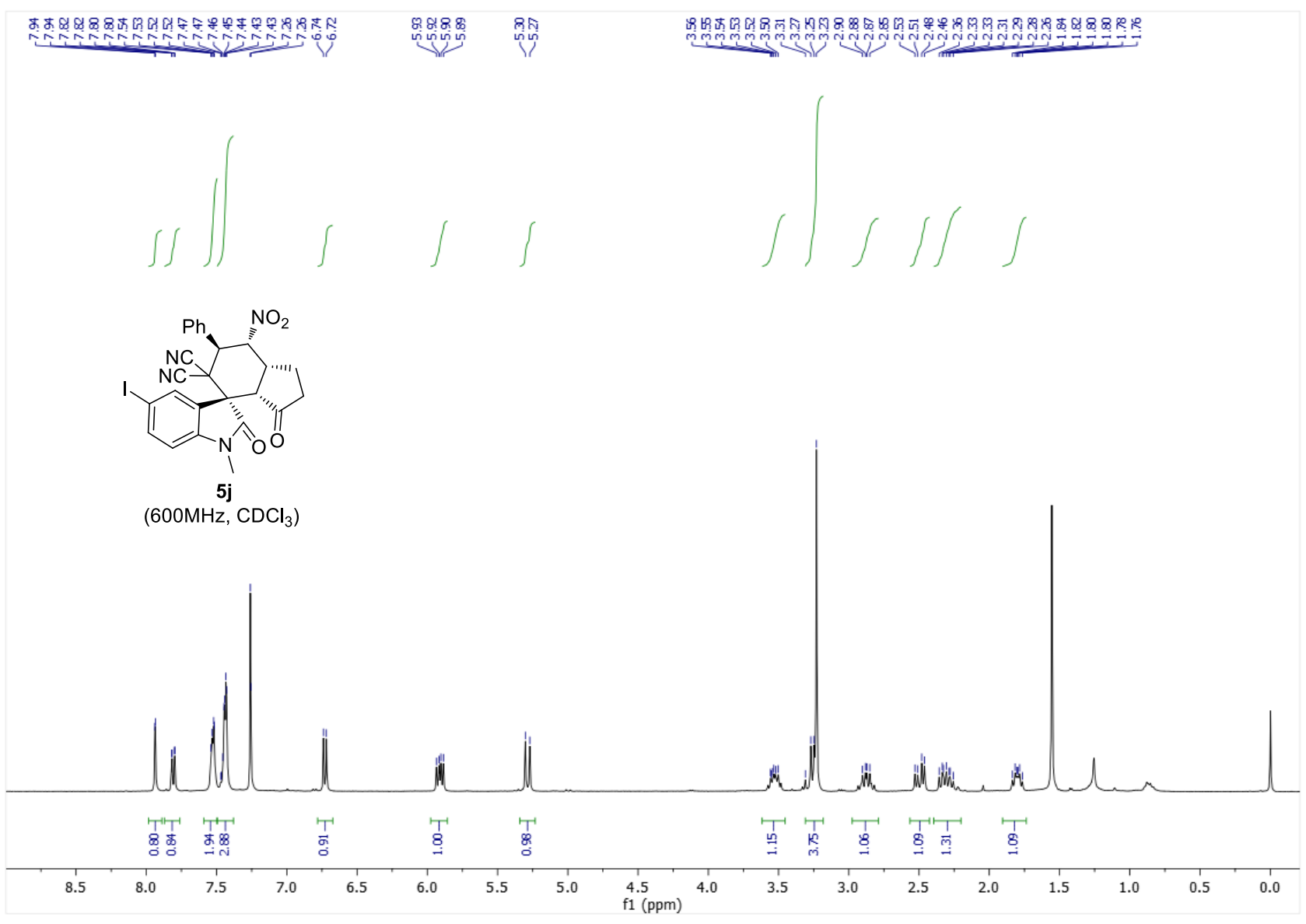

商

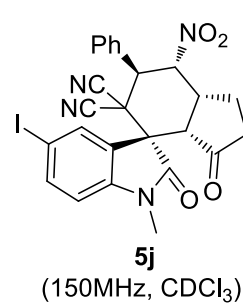

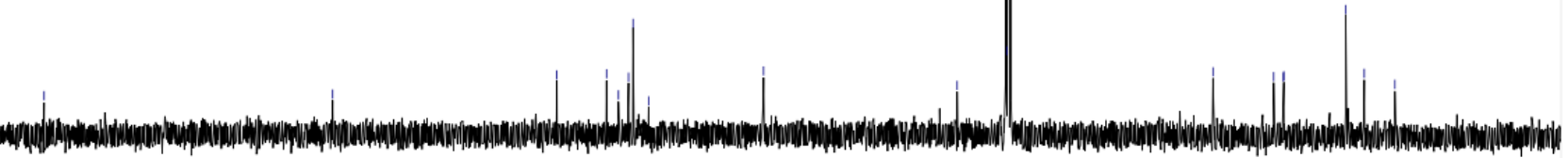

\begin{tabular}{lllllllllllllllllllllll}
\hline 210 & 200 & 190 & 180 & 170 & 160 & 150 & 140 & 130 & 120 & $\underset{\mathrm{f} 1(\mathrm{ppm})}{110}$ & 100 & 90 & 80 & 70 & 60 & 50 & 40 & 30 & 20 & 10 & 0
\end{tabular}




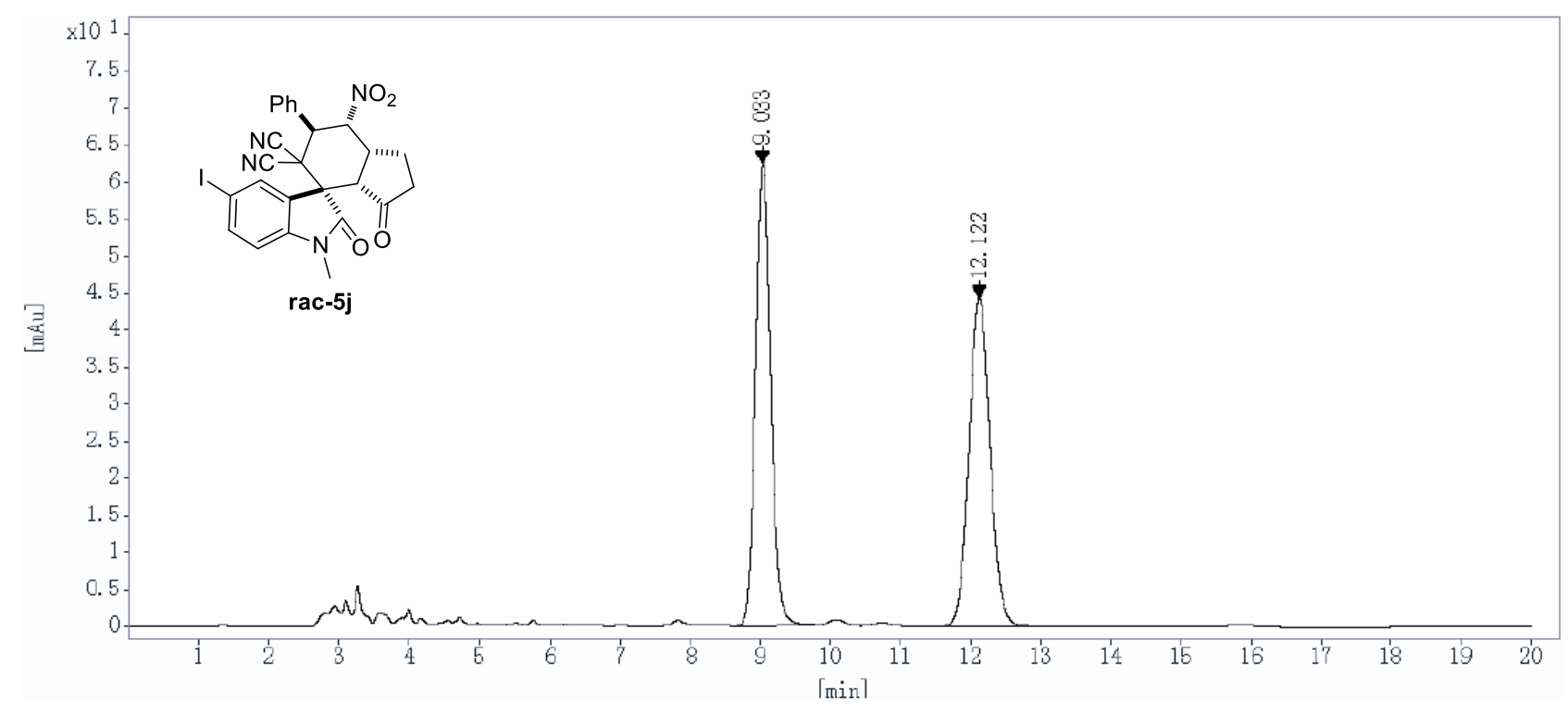

\begin{tabular}{cccccc}
$\begin{array}{c}\text { Ret Time } \\
\text { [min] }\end{array}$ & $\begin{array}{c}\text { Peak } \\
\text { Type }\end{array}$ & $\begin{array}{c}\text { Width } \\
\text { [min] }\end{array}$ & $\begin{array}{c}\text { Height } \\
\text { [mAU] }\end{array}$ & $\begin{array}{c}\text { Area } \\
{\left[\mathrm{mAU}^{*} \text { s] }\right.}\end{array}$ & $\begin{array}{c}\text { Area } \\
{[\%]}\end{array}$ \\
\hline 9.033 & BB & 0.23 & 62.2731 & 936.2390 & 50.2052 \\
12.122 & BB & 0.33 & 44.3350 & 928.5851 & 49.7948
\end{tabular}

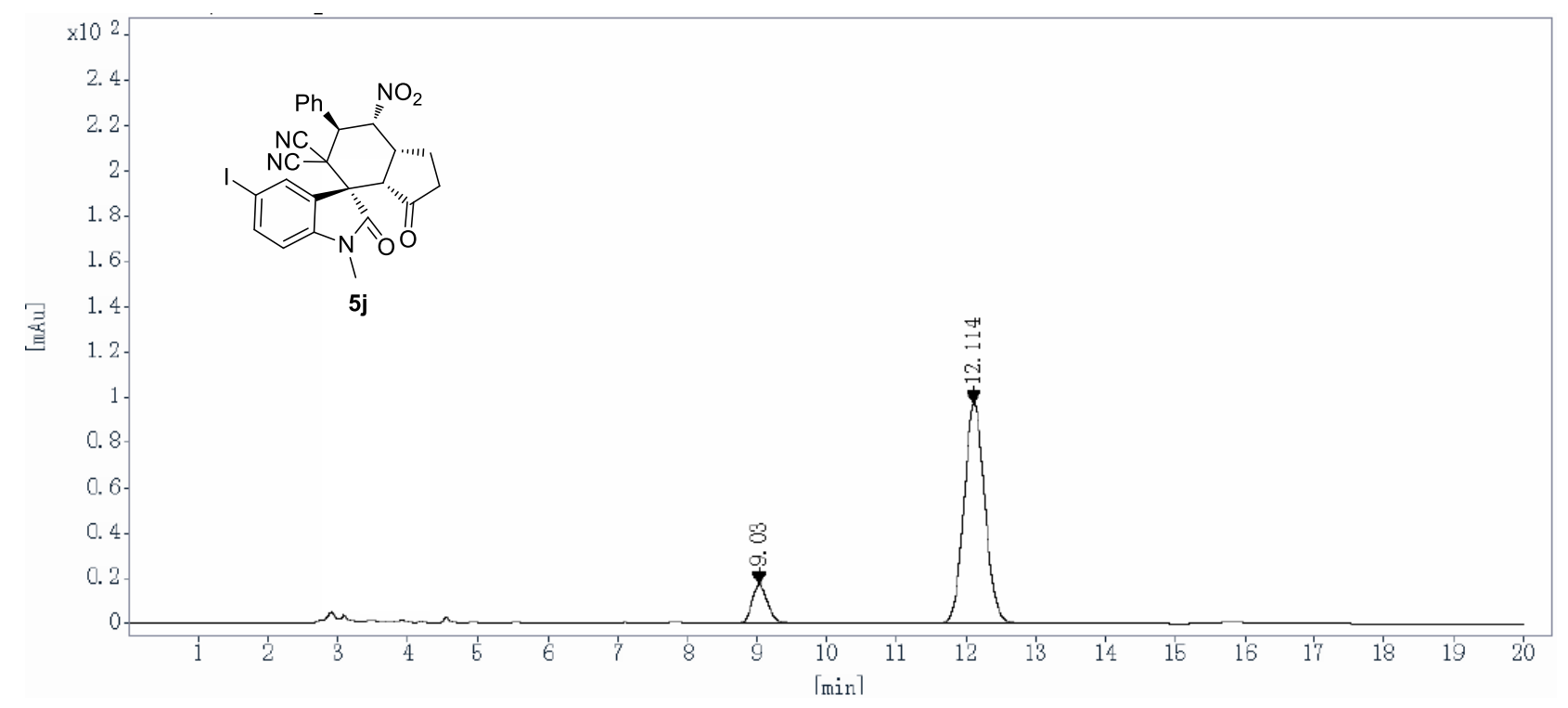

\begin{tabular}{cccccc}
$\begin{array}{c}\text { Ret Time } \\
\text { [min] }\end{array}$ & $\begin{array}{c}\text { Peak } \\
\text { Type }\end{array}$ & $\begin{array}{c}\text { Width } \\
\text { [min] }\end{array}$ & $\begin{array}{c}\text { Height } \\
{[\mathrm{mAU}]}\end{array}$ & $\begin{array}{c}\text { Area } \\
{[\mathrm{mAU} \text { *s] }}\end{array}$ & $\begin{array}{c}\text { Area } \\
{[\%]}\end{array}$ \\
\hline 9.030 & BBA & 0.24 & 17.1948 & 273.0152 & 11.7910 \\
12.114 & BB & 0.33 & 97.0805 & 2042.4358 & 88.2090
\end{tabular}



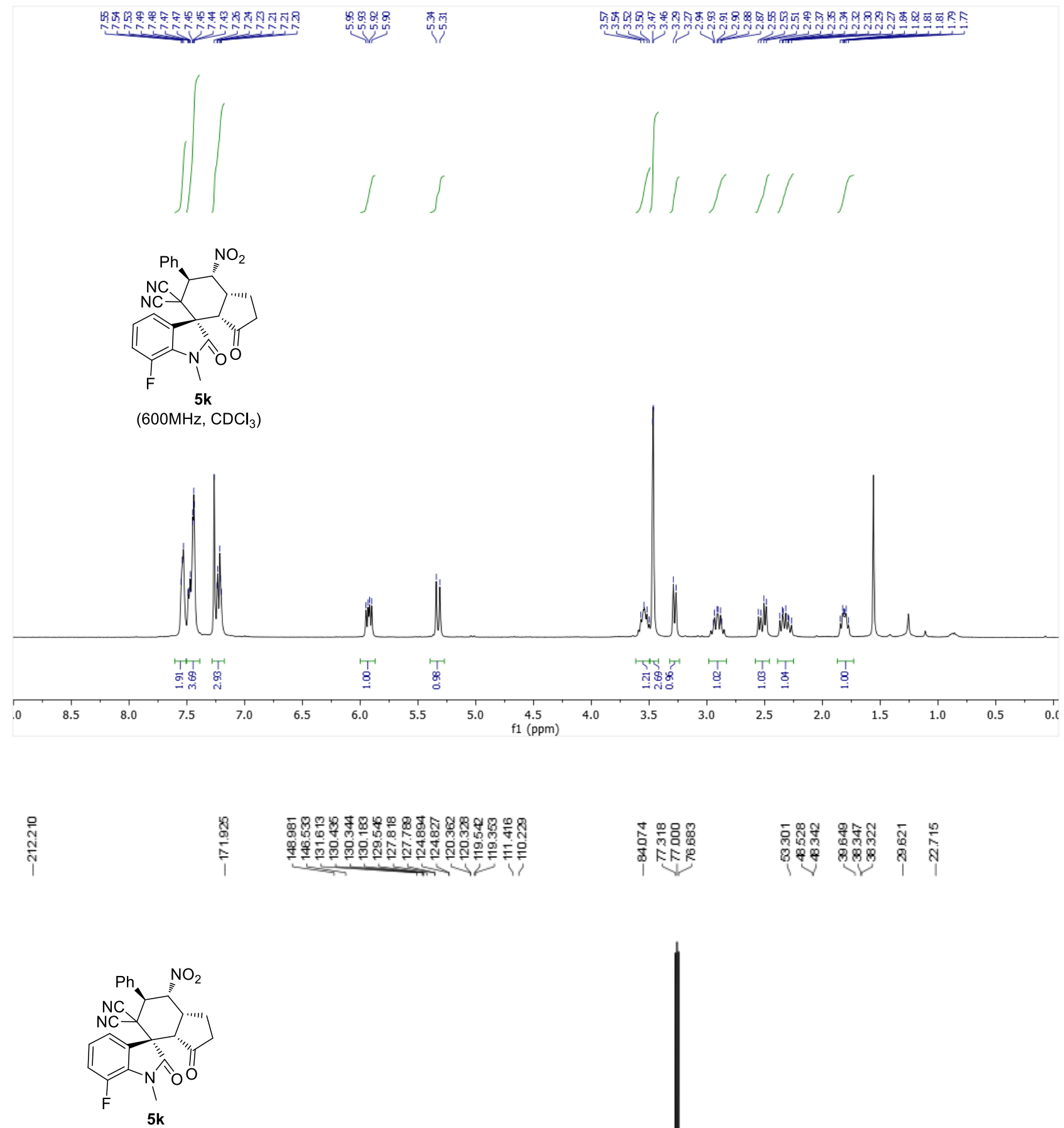

$\left(100 \mathrm{MHz}, \mathrm{CDCl}_{3}\right)$

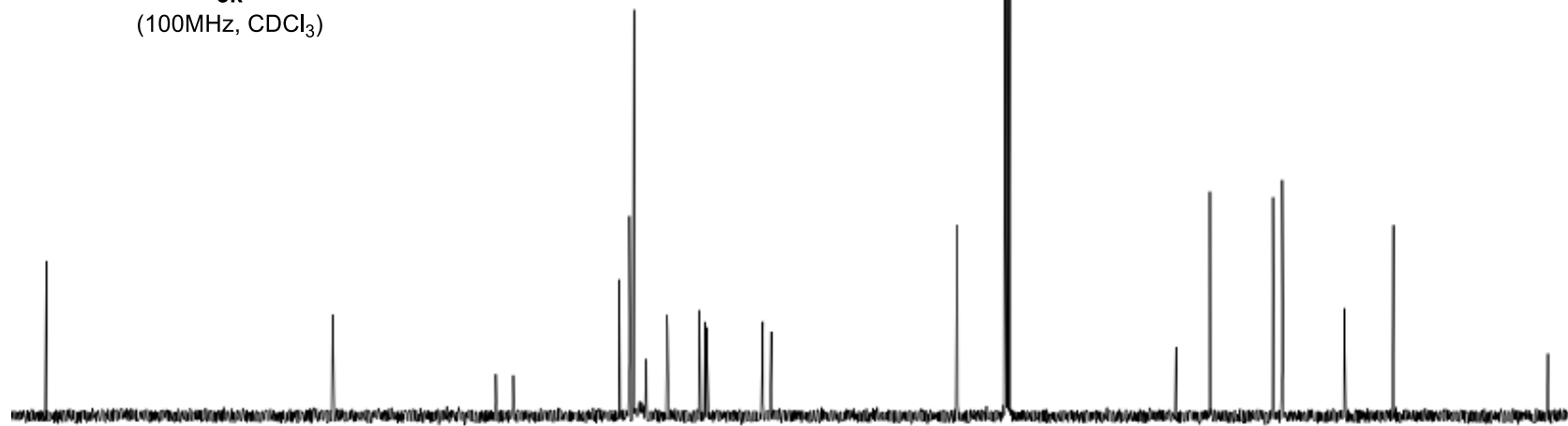

$\begin{array}{llllllllllllllllllllll}210 & 200 & 190 & 180 & 170 & 160 & 150 & 140 & 130 & 120 & \underset{f 1}{110}(\mathrm{ppm}) & 100 & 90 & 80 & 70 & 60 & 50 & 40 & 30 & 20 & 10 & 0\end{array}$ 


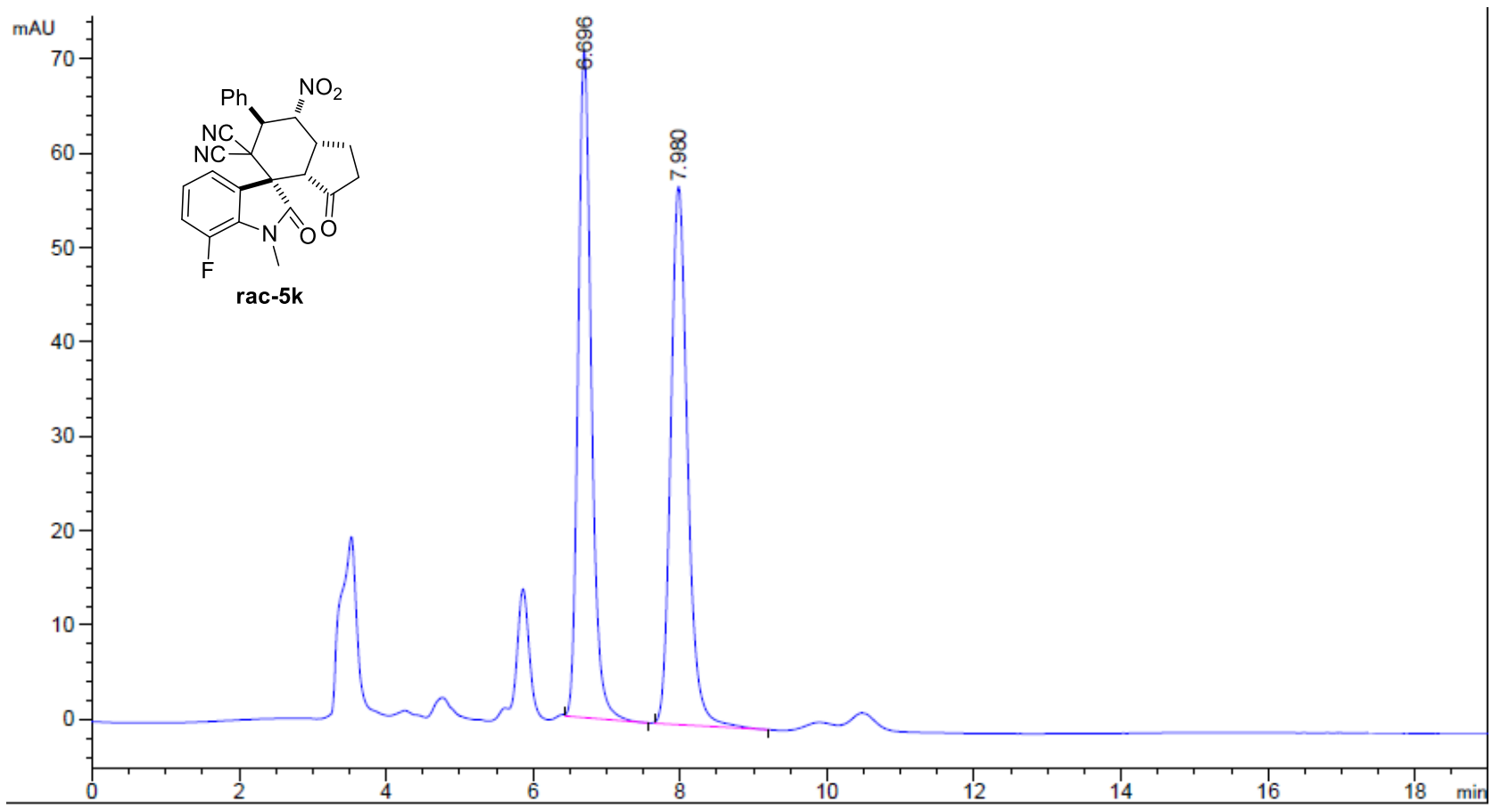

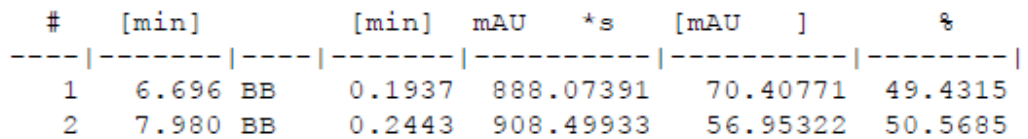
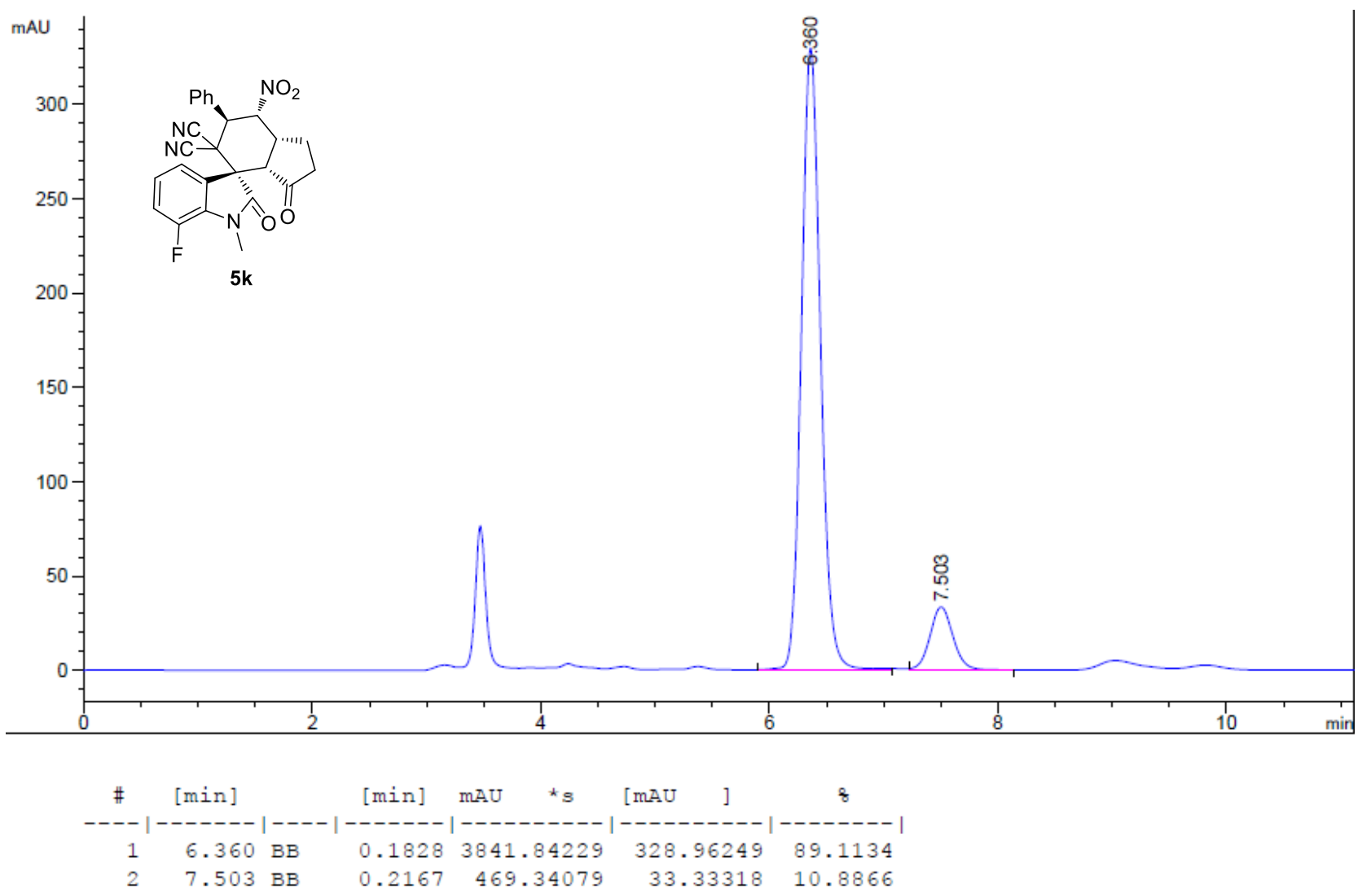


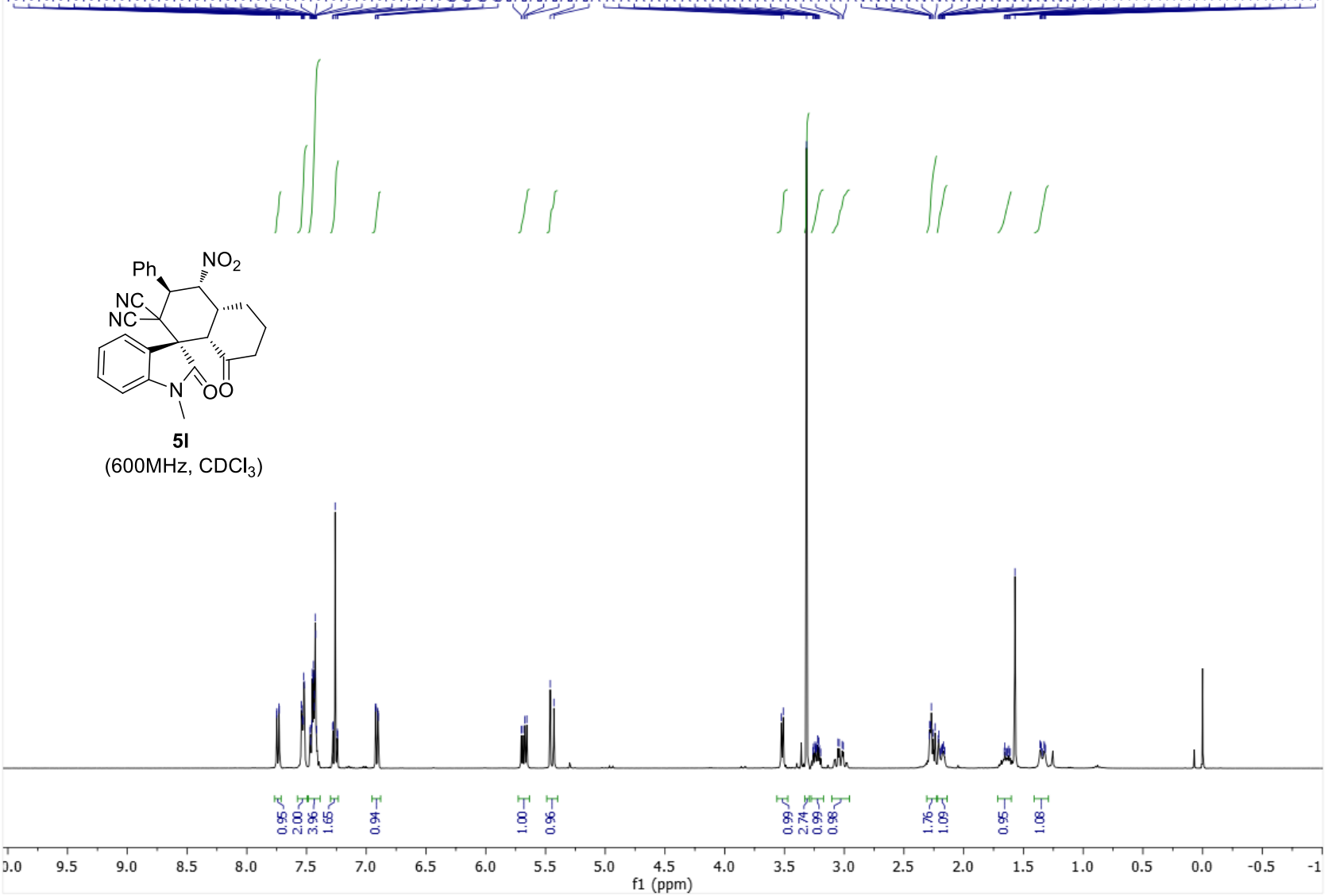

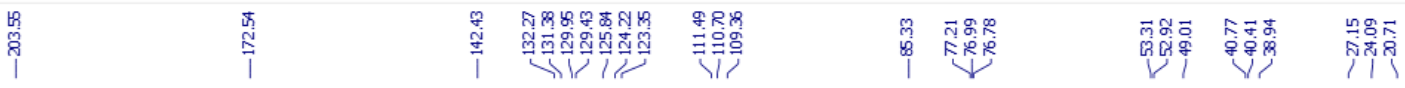

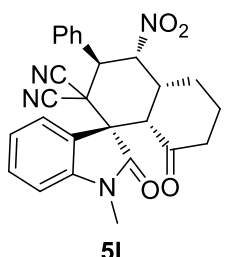

$\left(150 \mathrm{MHz}, \mathrm{CDCl}_{3}\right)$

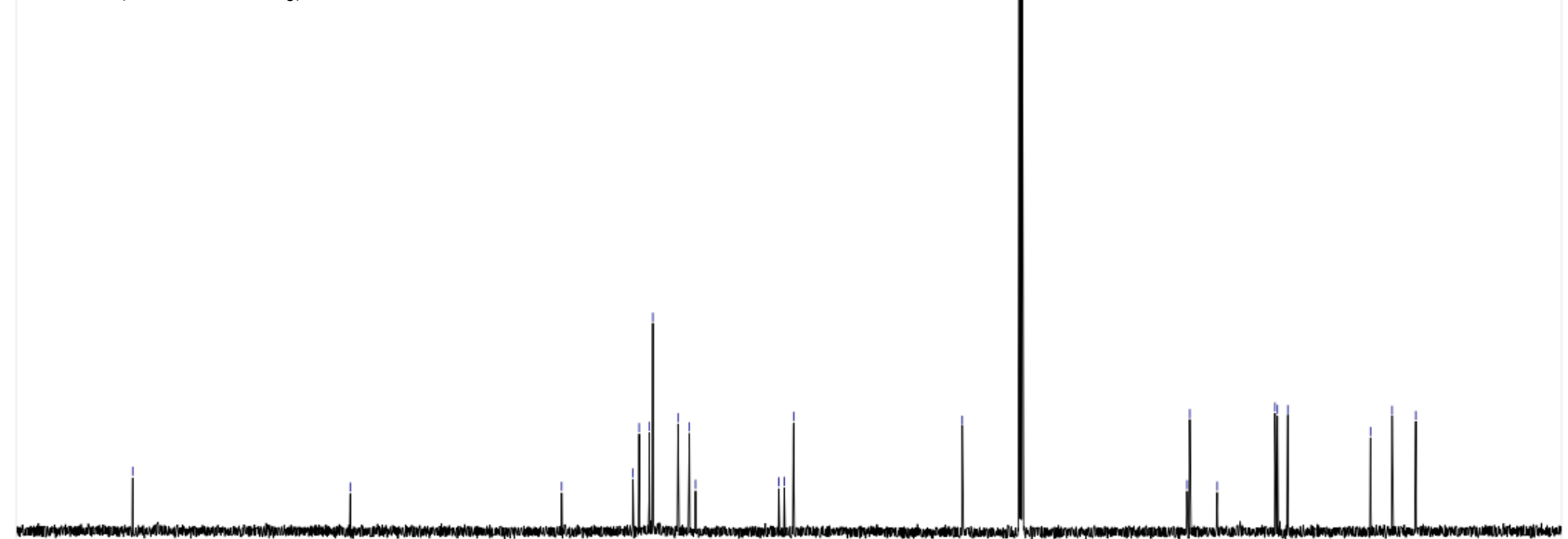

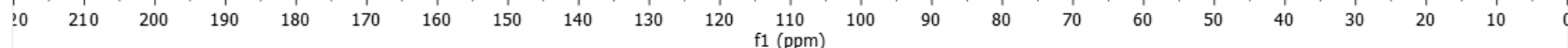



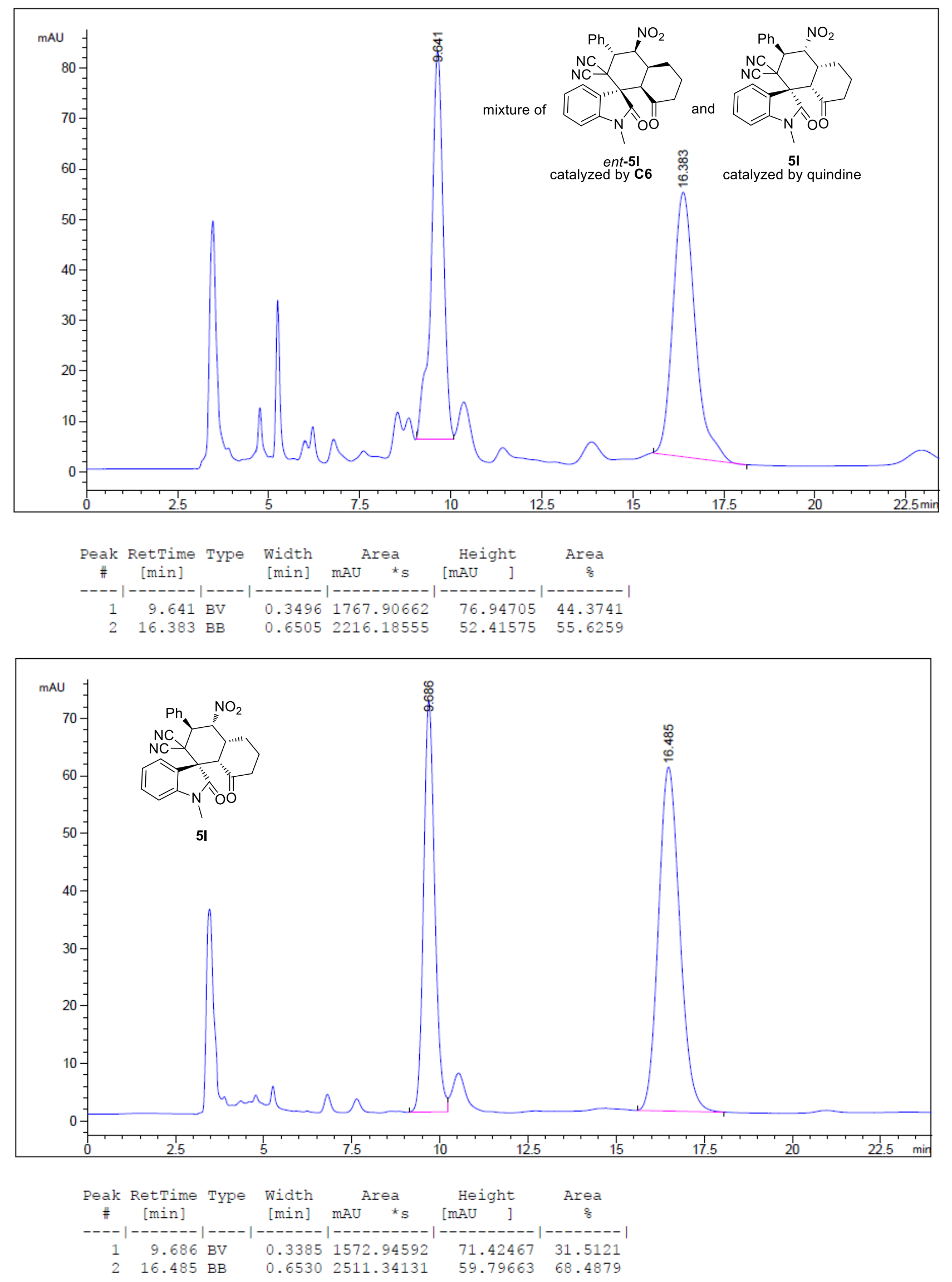

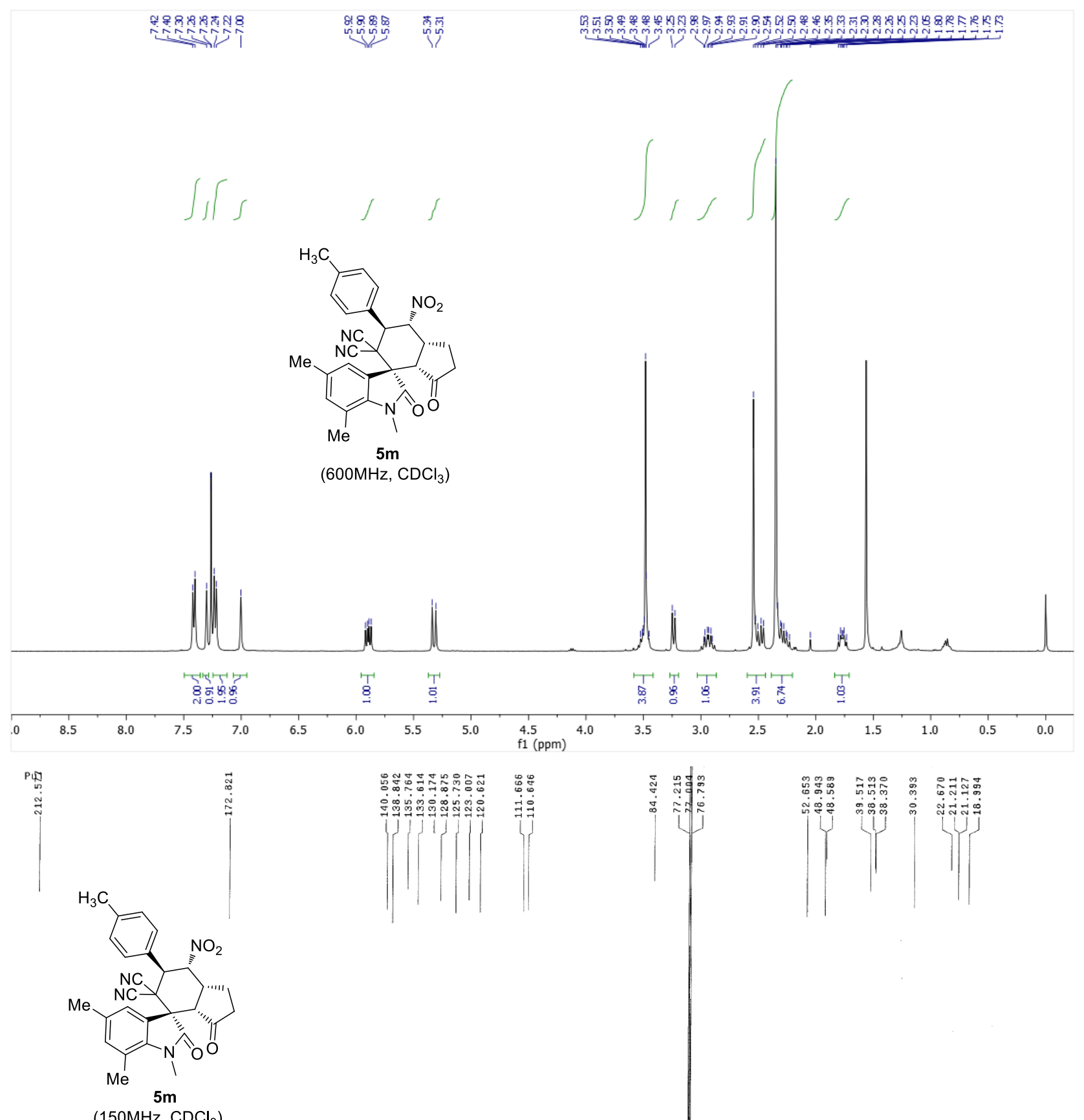

$\left(150 \mathrm{MHz}, \mathrm{CDCl}_{3}\right)$

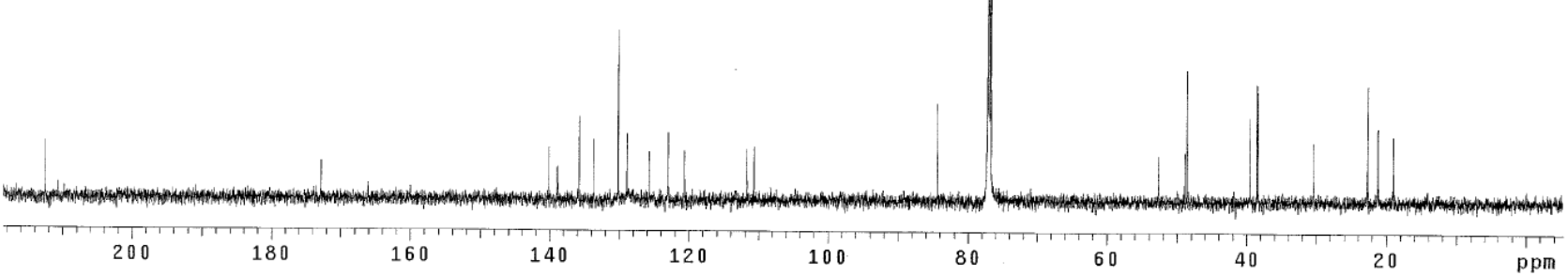




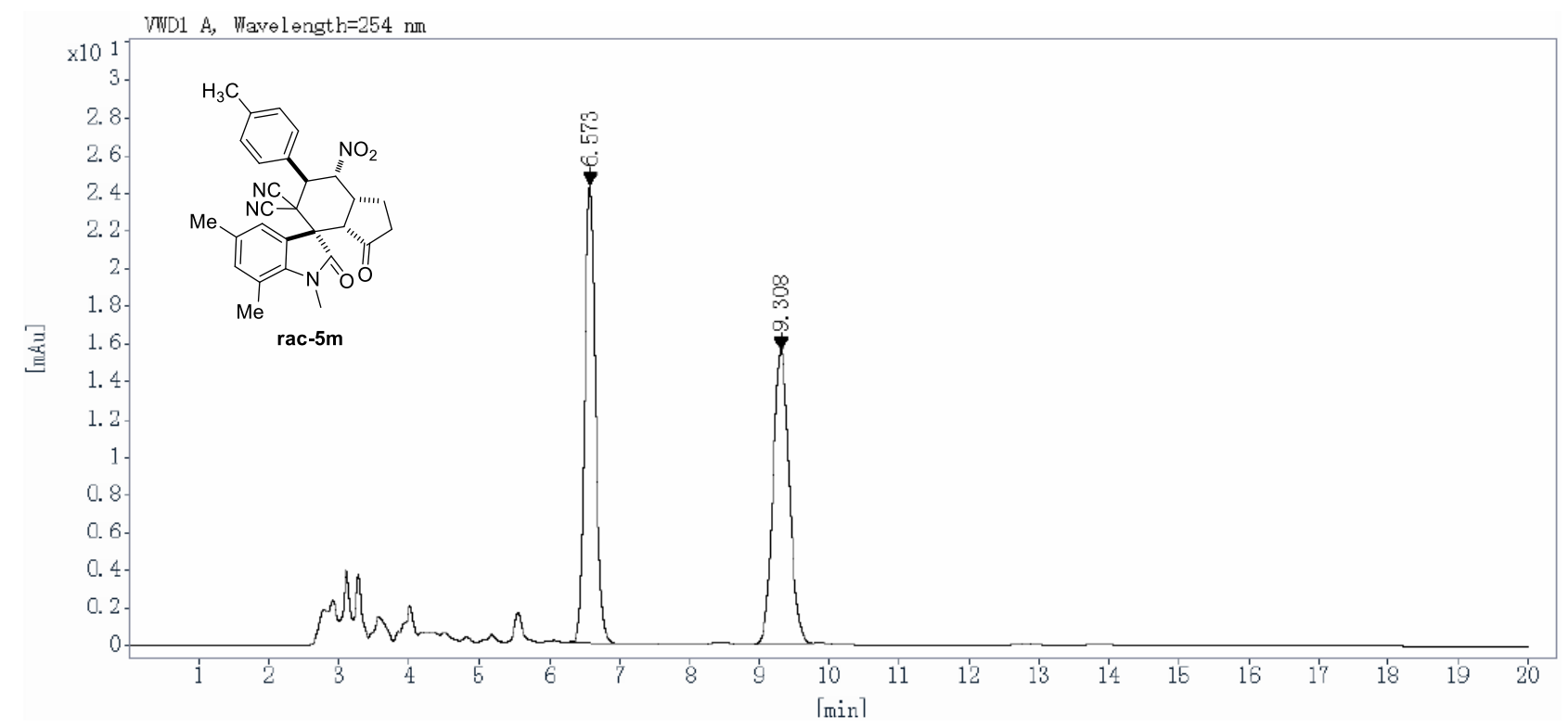

$\begin{array}{cccccc}\begin{array}{c}\text { Ret Time } \\ \text { [min] }\end{array} & \begin{array}{c}\text { Peak } \\ \text { Type }\end{array} & \begin{array}{c}\text { Width } \\ \text { [min] }\end{array} & \begin{array}{c}\text { Height } \\ \text { [mAU] }\end{array} & \begin{array}{c}\text { Area } \\ {\left[\mathbf{m A U}^{*} \text { s] }\right.}\end{array} & \begin{array}{c}\text { Area } \\ {[\%]}\end{array} \\ 6.573 & \text { BB } & 0.16 & 24.2341 & 255.2037 & 50.1757 \\ 9.308 & \text { BB } & 0.25 & 15.5648 & 253.4164 & 49.8243\end{array}$

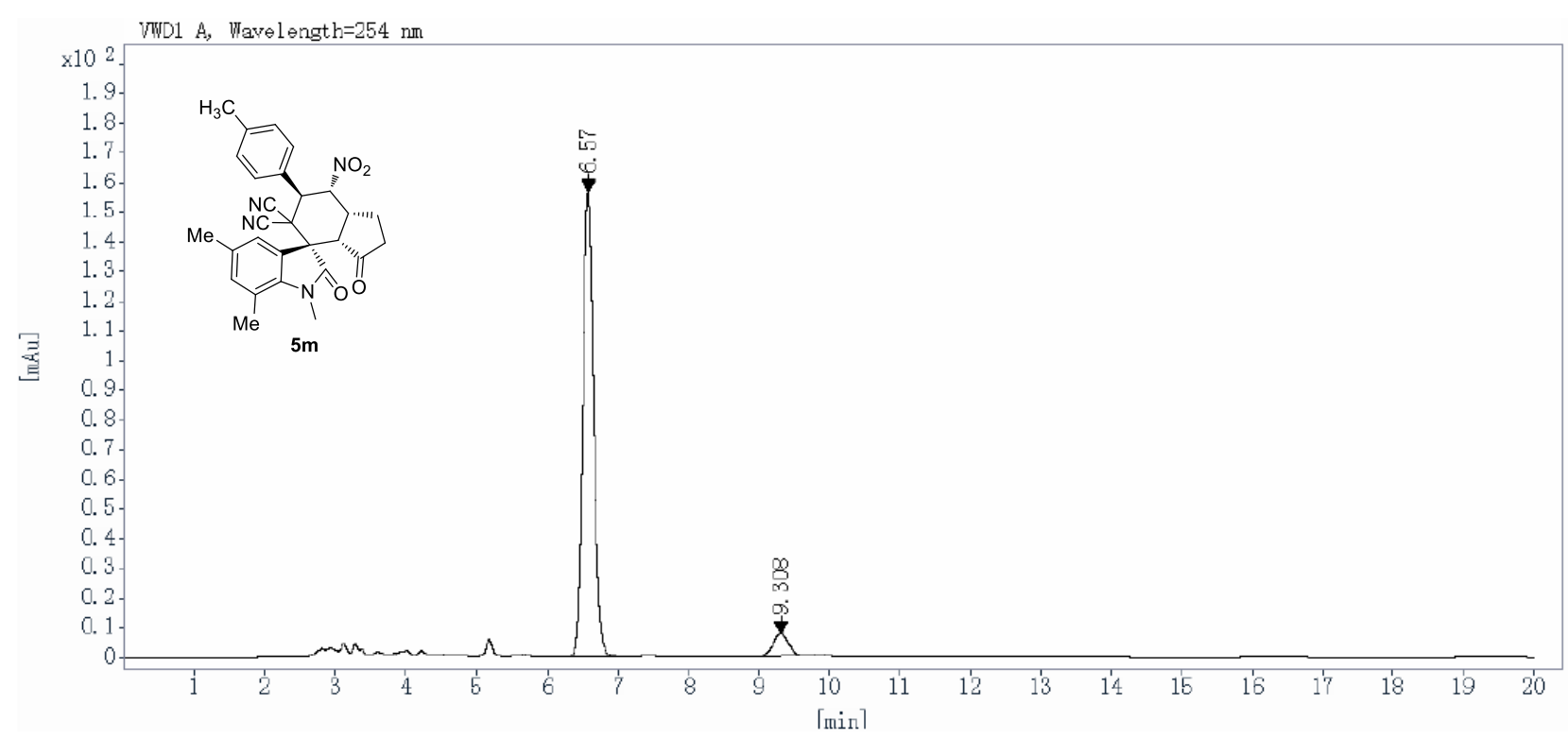

$\begin{array}{cccccc}\begin{array}{c}\text { Ret Time } \\ \text { [min] }\end{array} & \begin{array}{c}\text { Peak } \\ \text { Type }\end{array} & \begin{array}{c}\text { Width } \\ {[\mathrm{min}]}\end{array} & \begin{array}{c}\text { Height } \\ {[\mathrm{mAU}]}\end{array} & \begin{array}{c}\text { Area } \\ {\left[\mathrm{mAU}^{*} \mathbf{s}\right]}\end{array} & \begin{array}{c}\text { Area } \\ {[\%]}\end{array} \\ 6.570 & \text { BB } & 0.16 & 156.4582 & 1636.0673 & 93.1627 \\ 9.308 & \text { BB } & 0.25 & 7.6018 & 120.0720 & 6.8373\end{array}$



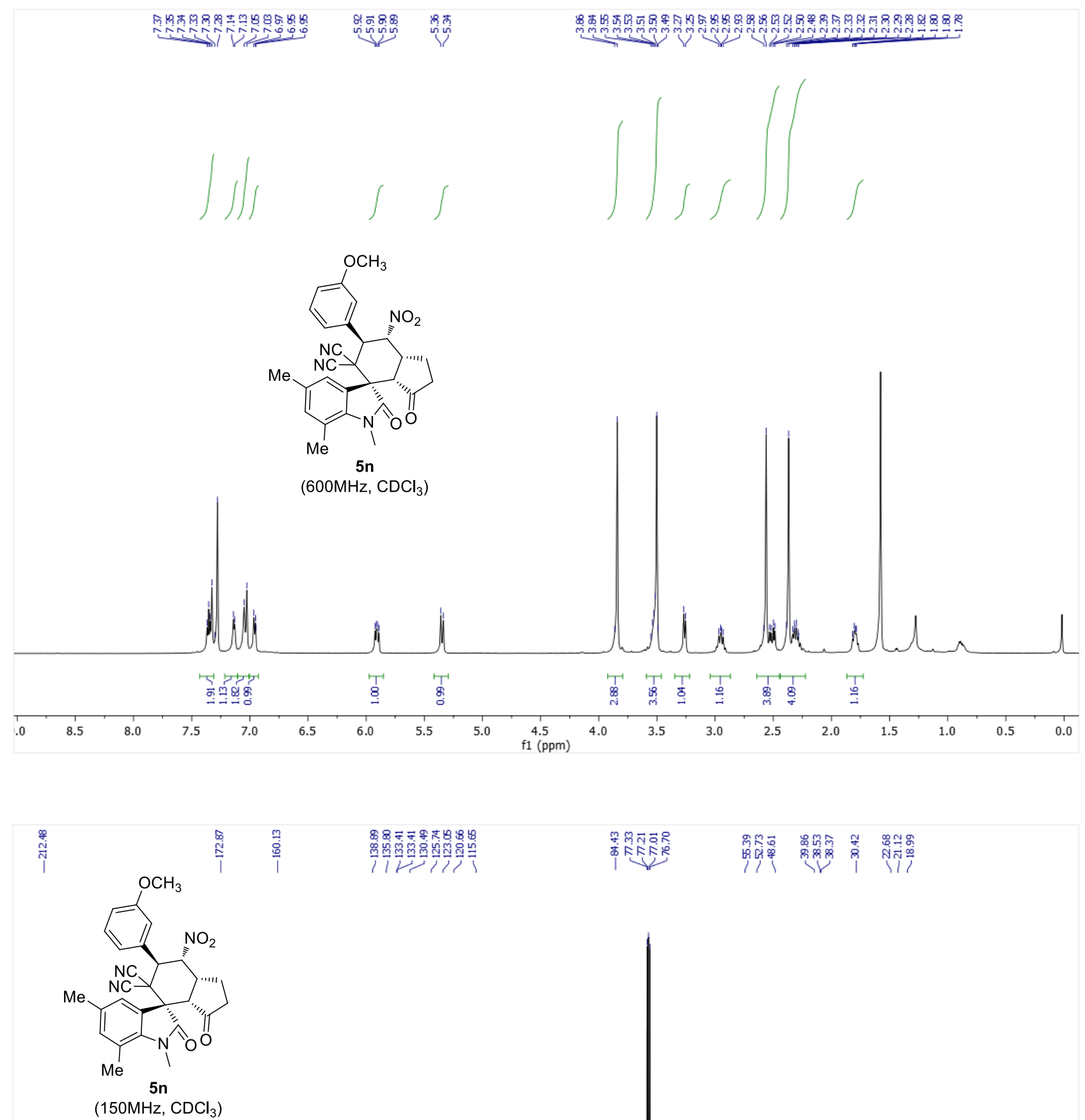

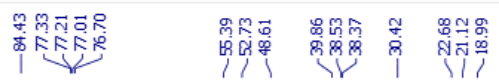

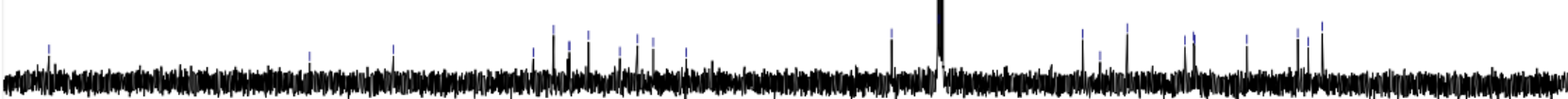



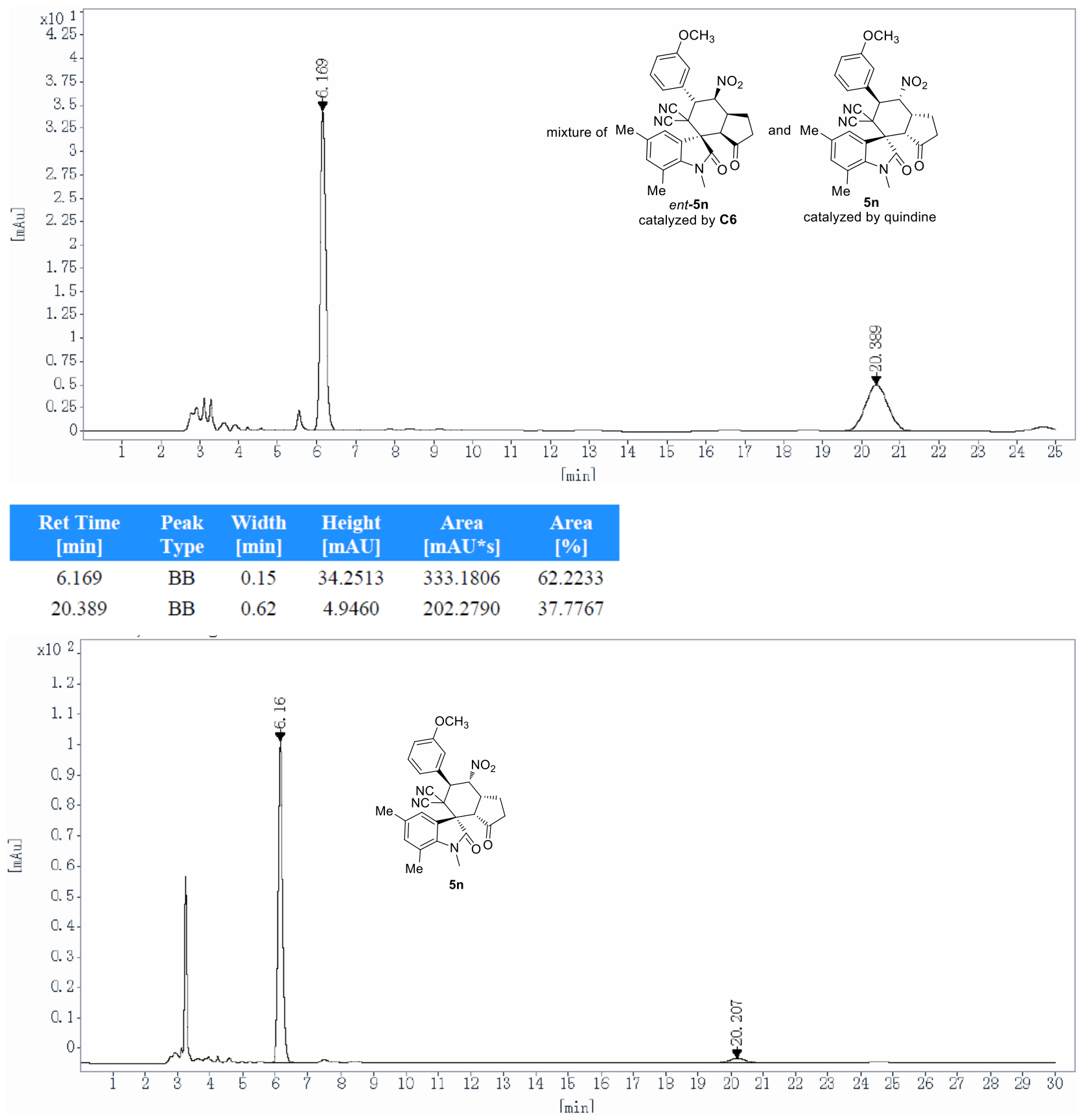

\begin{tabular}{cccccc}
$\begin{array}{c}\text { Ret Time } \\
\text { [min] }\end{array}$ & $\begin{array}{c}\text { Peak } \\
\text { Type }\end{array}$ & $\begin{array}{c}\text { Width } \\
{[\mathrm{min}]}\end{array}$ & $\begin{array}{c}\text { Height } \\
{[\mathrm{mAU}]}\end{array}$ & $\begin{array}{c}\text { Area } \\
{[\mathrm{mAU} \text { *s] }}\end{array}$ & $\begin{array}{c}\text { Area } \\
{[\%]}\end{array}$ \\
\hline 6.160 & BB & 0.15 & 105.7407 & 1029.4650 & 94.0840 \\
20.207 & BB & 0.58 & 1.6240 & 64.7330 & 5.9160
\end{tabular}




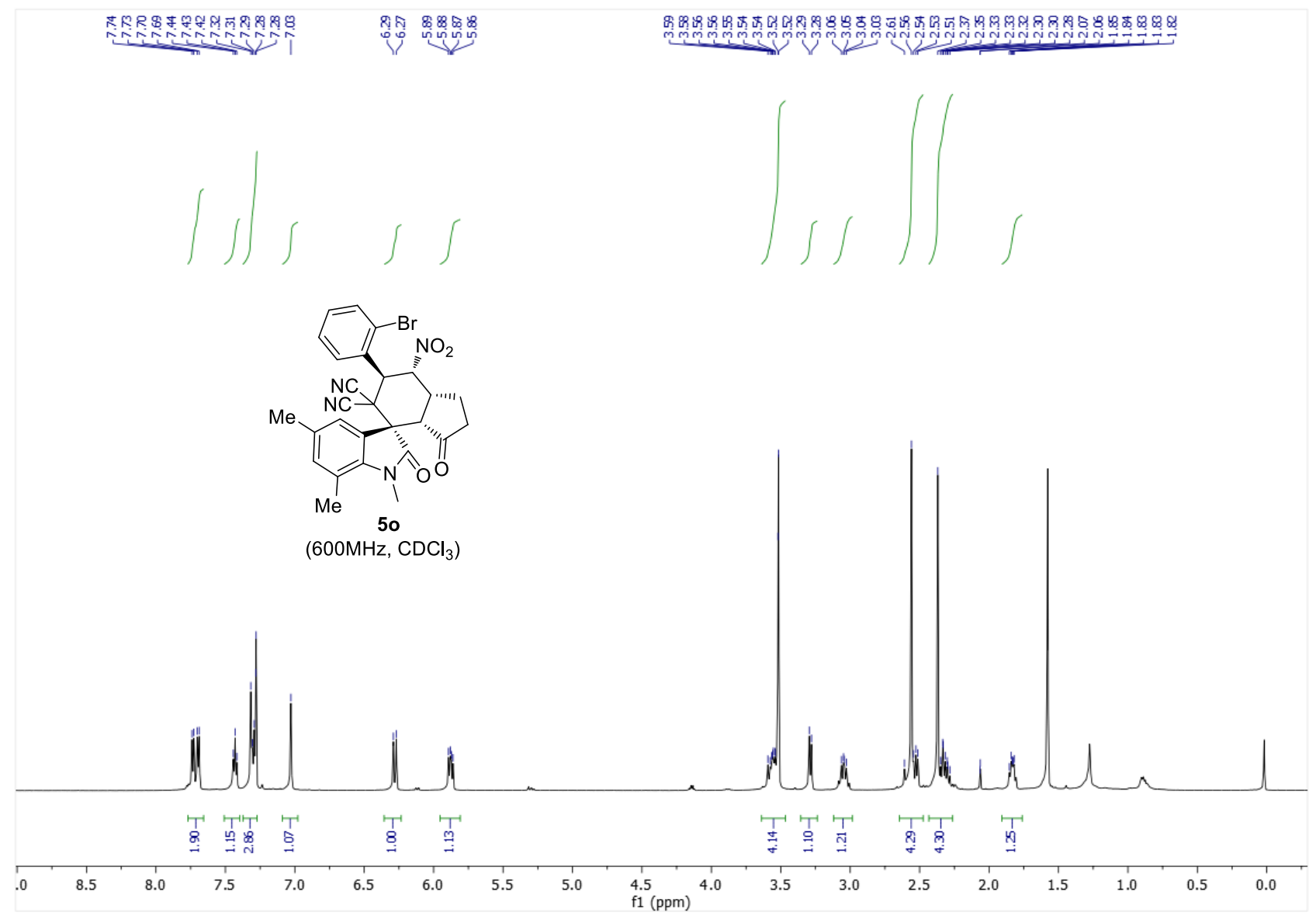

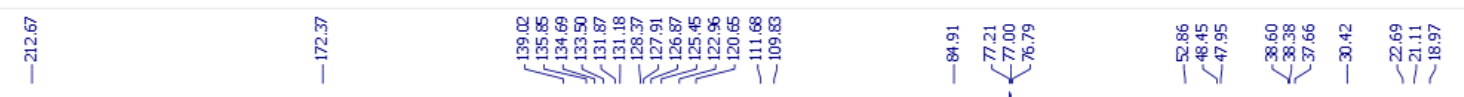
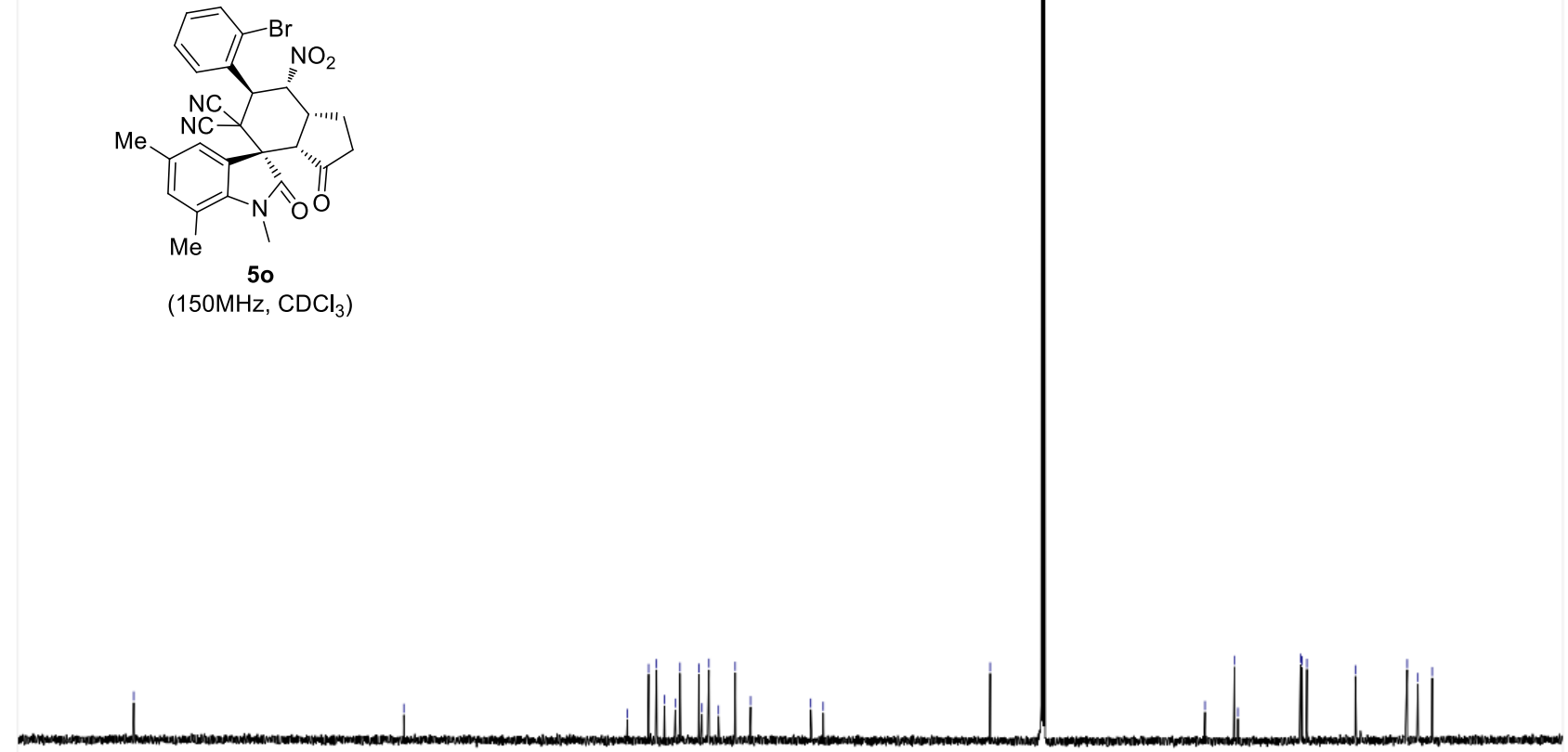

$\begin{array}{lllllllllll}220 & 210 & 200 & 190 & 180 & 170 & 160 & 150 & 140 & 130 & \begin{array}{c}120 \\ \mathrm{f} 1(\mathrm{ppm})\end{array}\end{array}$ 


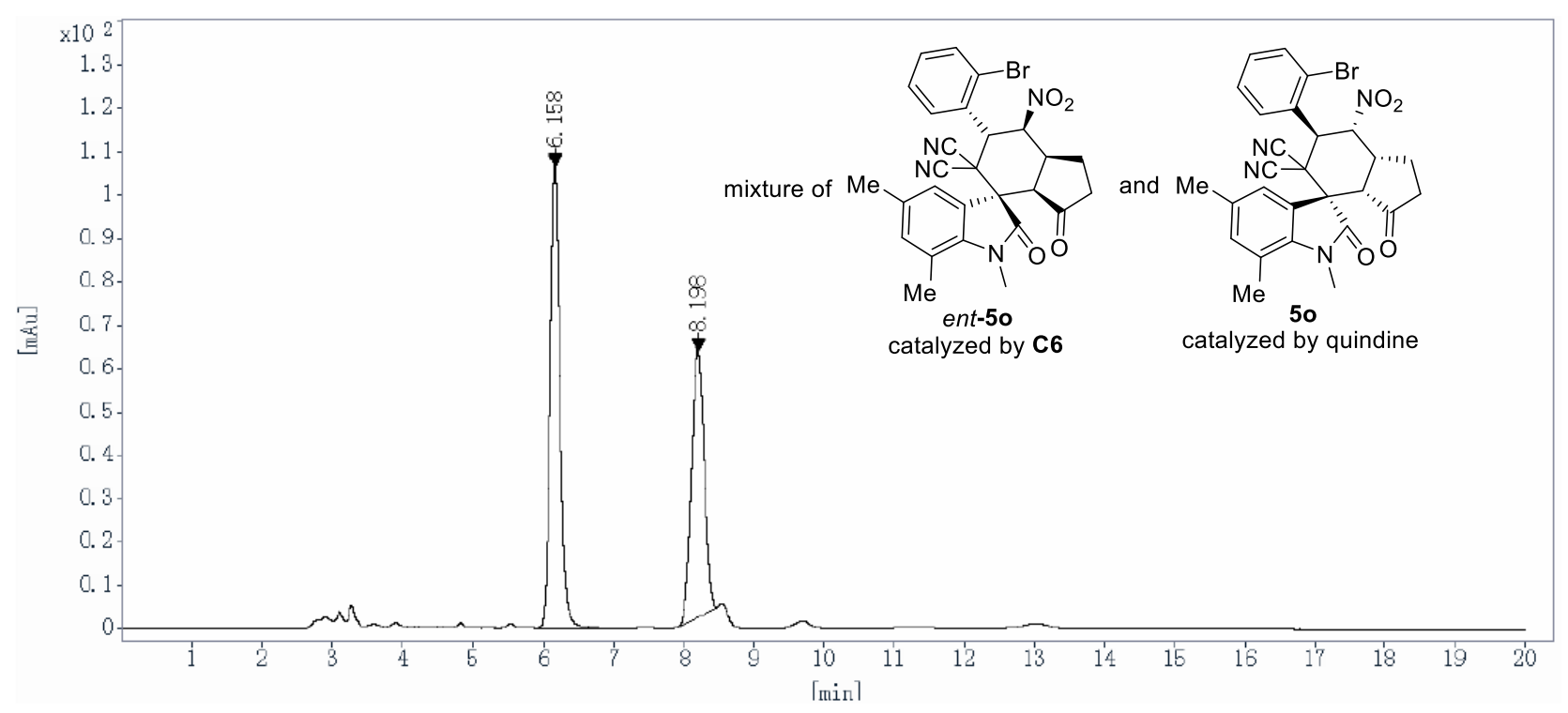

\begin{tabular}{cccccc}
$\begin{array}{c}\text { Ret Time } \\
\text { [min] }\end{array}$ & $\begin{array}{c}\text { Peak } \\
\text { Type }\end{array}$ & $\begin{array}{c}\text { Width } \\
{[\mathrm{min}]}\end{array}$ & $\begin{array}{c}\text { Height } \\
{[\mathrm{mAU}]}\end{array}$ & $\begin{array}{c}\text { Area } \\
{\left[\mathrm{mAU}^{*} \text { s] }\right.}\end{array}$ & $\begin{array}{c}\text { Area } \\
{[\%]}\end{array}$ \\
\hline 6.158 & VB & 0.15 & 106.4596 & 1023.8345 & 56.8231 \\
8.198 & BB & 0.20 & 61.4051 & 777.9598 & 43.1769
\end{tabular}

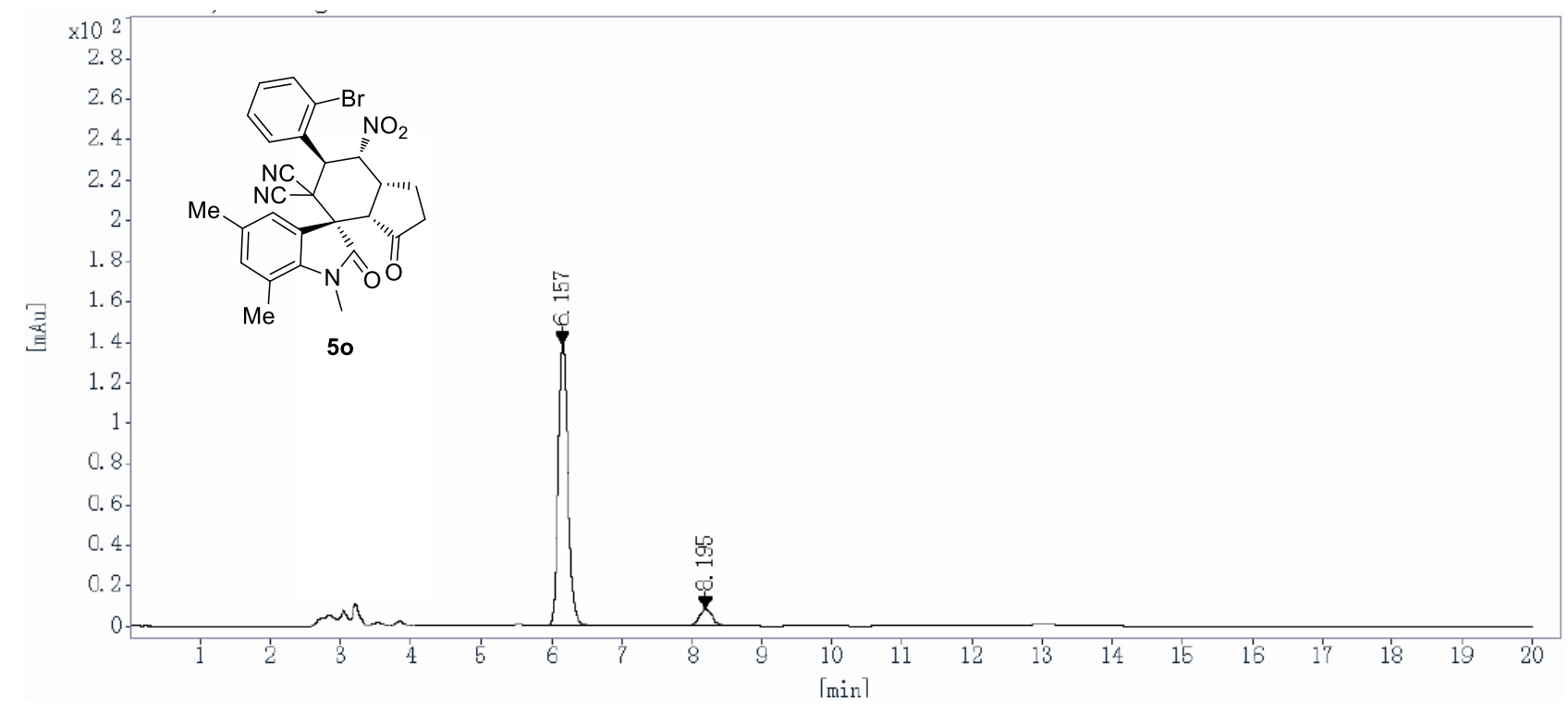

\begin{tabular}{cccccc}
$\begin{array}{c}\text { Ret Time } \\
\text { [min] }\end{array}$ & $\begin{array}{c}\text { Peak } \\
\text { Type }\end{array}$ & $\begin{array}{c}\text { Width } \\
{[\mathrm{min}]}\end{array}$ & $\begin{array}{c}\text { Height } \\
{[\mathrm{mAU}]}\end{array}$ & $\begin{array}{c}\text { Area } \\
{\left[\mathrm{mAU}^{*} \mathbf{A}\right]}\end{array}$ & $\begin{array}{c}\text { Area } \\
{[\%]}\end{array}$ \\
\hline 6.157 & BB & 0.15 & 139.4192 & 1330.5614 & 92.5702 \\
8.195 & BBA & 0.20 & 8.1840 & 106.7920 & 7.4298
\end{tabular}



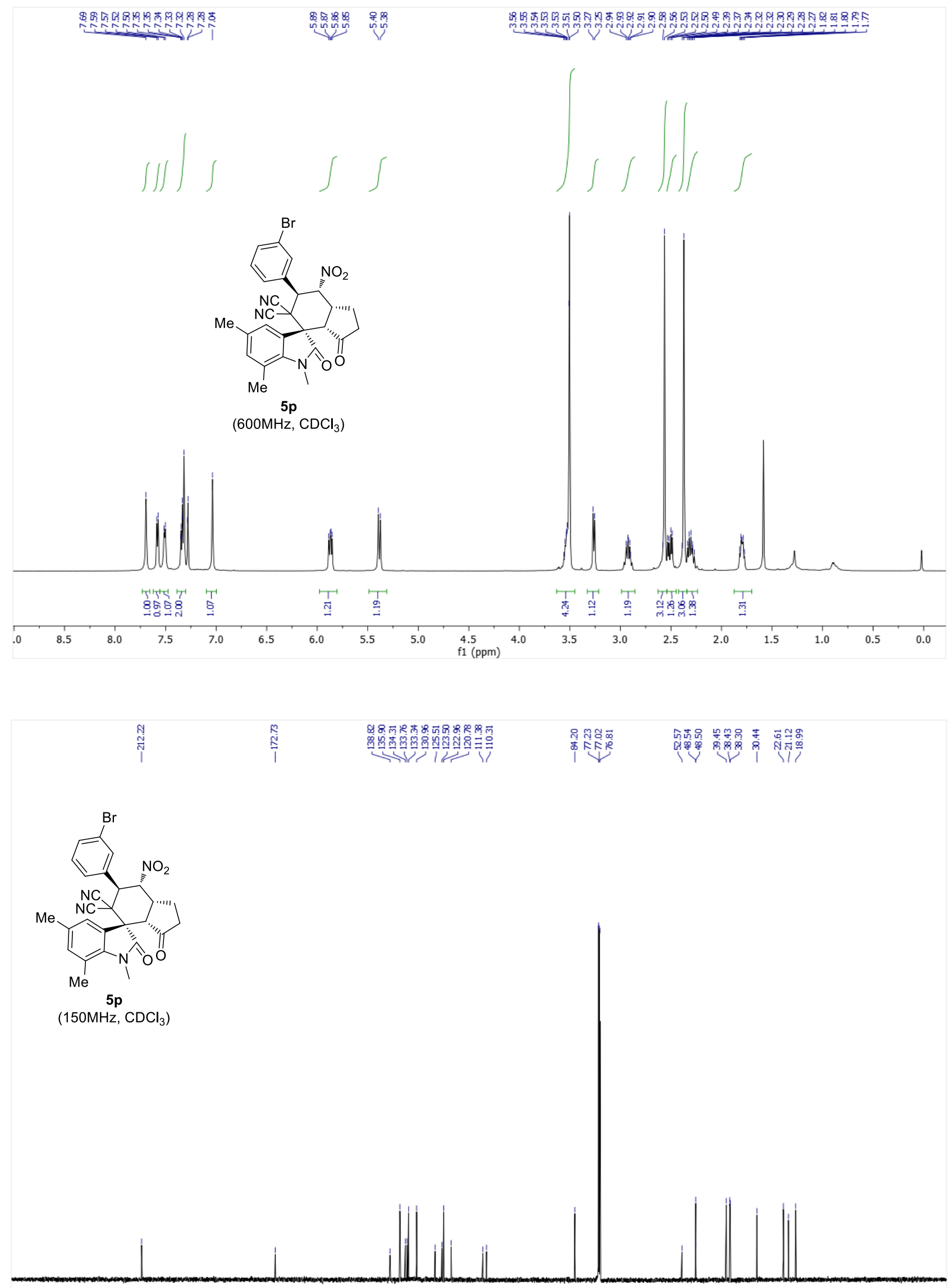

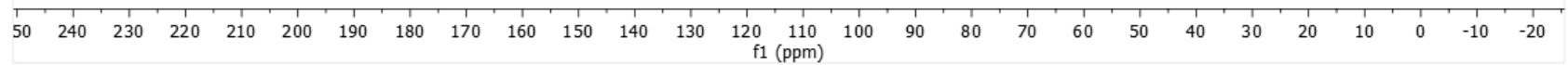




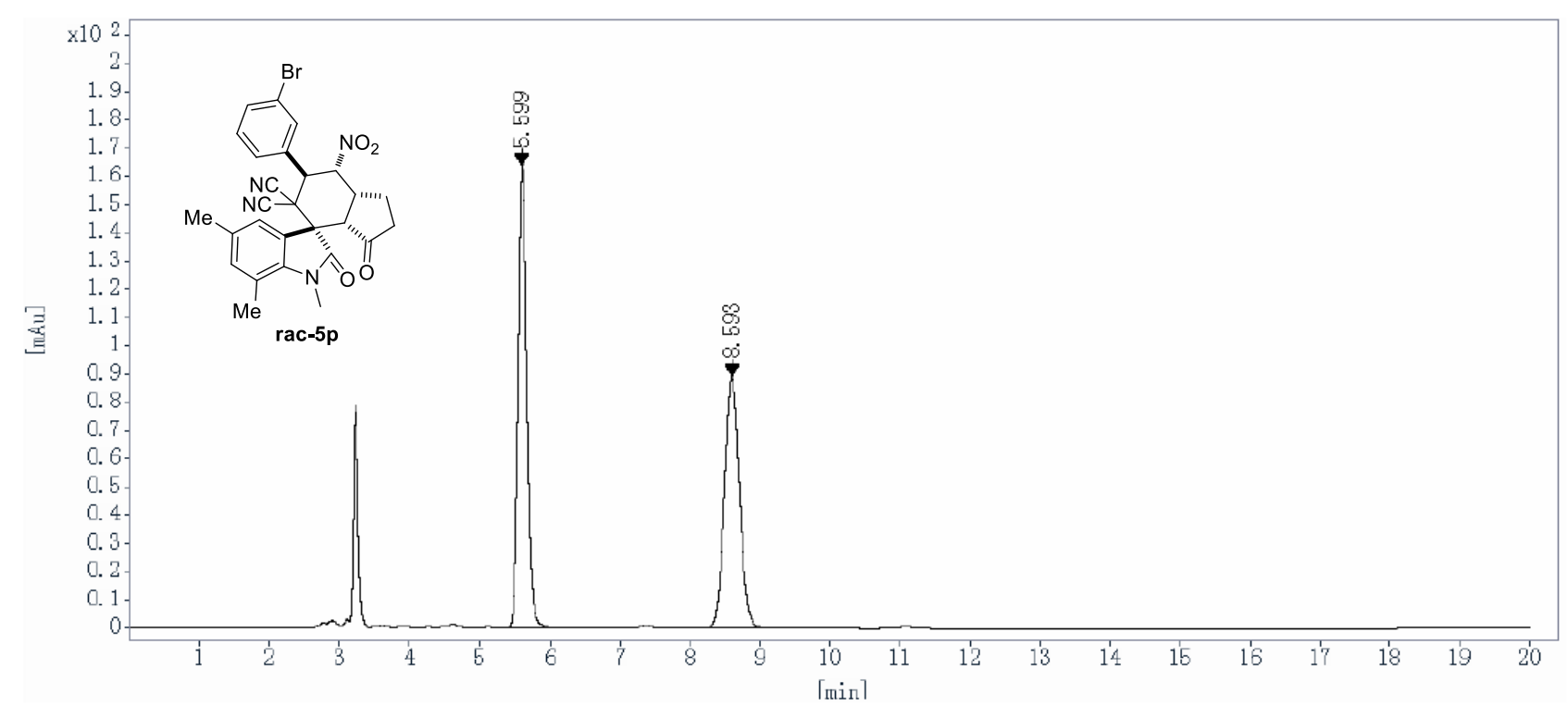

\begin{tabular}{cccccc}
$\begin{array}{c}\text { Ret Time } \\
\text { [min] }\end{array}$ & $\begin{array}{c}\text { Peak } \\
\text { Type }\end{array}$ & $\begin{array}{c}\text { Width } \\
\text { [min] }\end{array}$ & $\begin{array}{c}\text { Height } \\
\text { [mAU] }\end{array}$ & $\begin{array}{c}\text { Area } \\
{\left[\mathrm{mAU}^{*} \text { s] }\right.}\end{array}$ & $\begin{array}{c}\text { Area } \\
{[\%]}\end{array}$ \\
\hline 5.599 & BB & 0.13 & 163.3382 & 1419.0177 & 51.7719 \\
8.593 & BB & 0.23 & 88.9735 & 1321.8844 & 48.2281
\end{tabular}

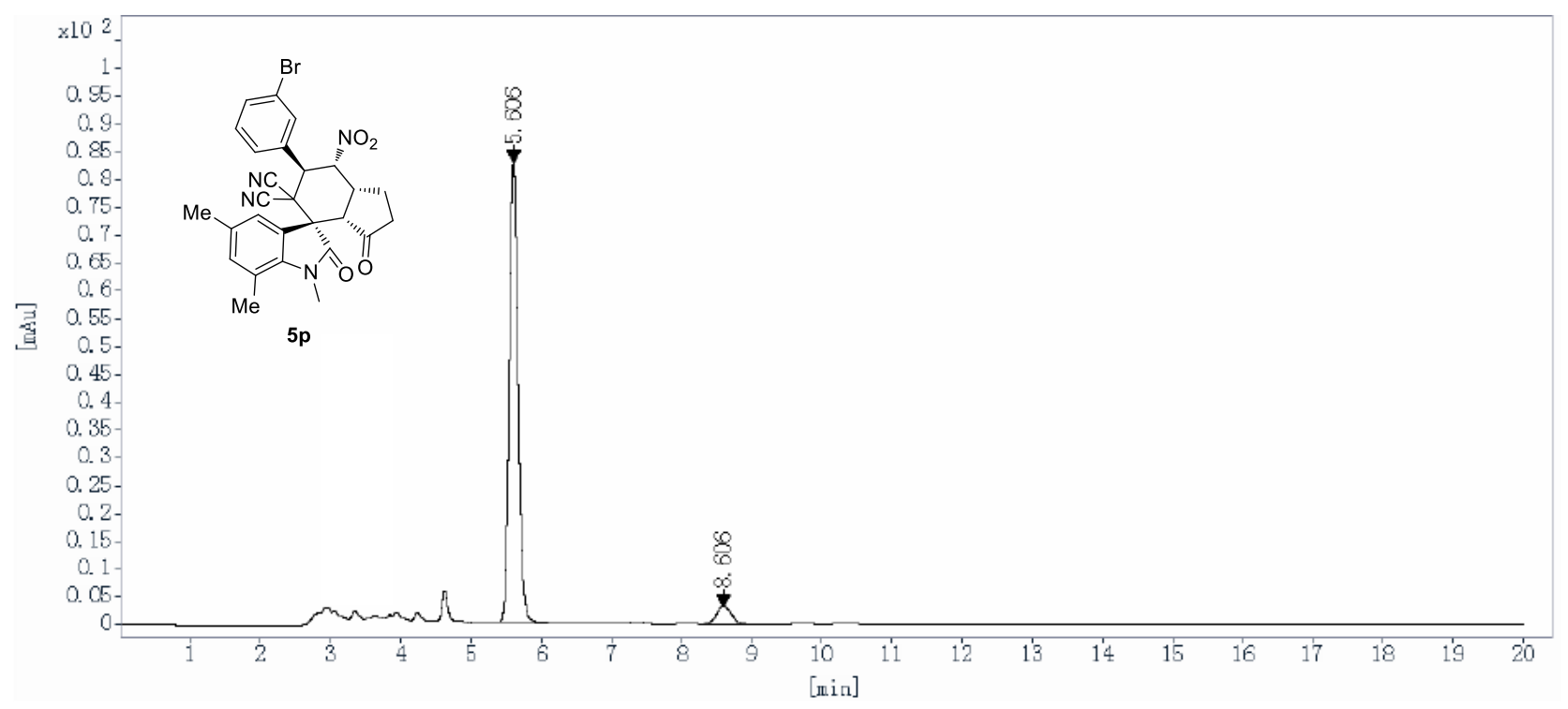

\begin{tabular}{cccccc}
$\begin{array}{c}\text { Ret Time } \\
{[\mathrm{min}]}\end{array}$ & $\begin{array}{c}\text { Peak } \\
\text { Type }\end{array}$ & $\begin{array}{c}\text { Width } \\
{[\mathrm{min}]}\end{array}$ & $\begin{array}{c}\text { Height } \\
{[\mathrm{mAU}]}\end{array}$ & $\begin{array}{c}\text { Area } \\
{[\mathrm{mAU} \text { *s] }}\end{array}$ & $\begin{array}{c}\text { Area } \\
{[\%]}\end{array}$ \\
\hline 5.606 & BB & 0.13 & 82.6674 & 716.1127 & 93.8489 \\
8.606 & BB & 0.23 & 3.1776 & 46.9359 & 6.1511
\end{tabular}



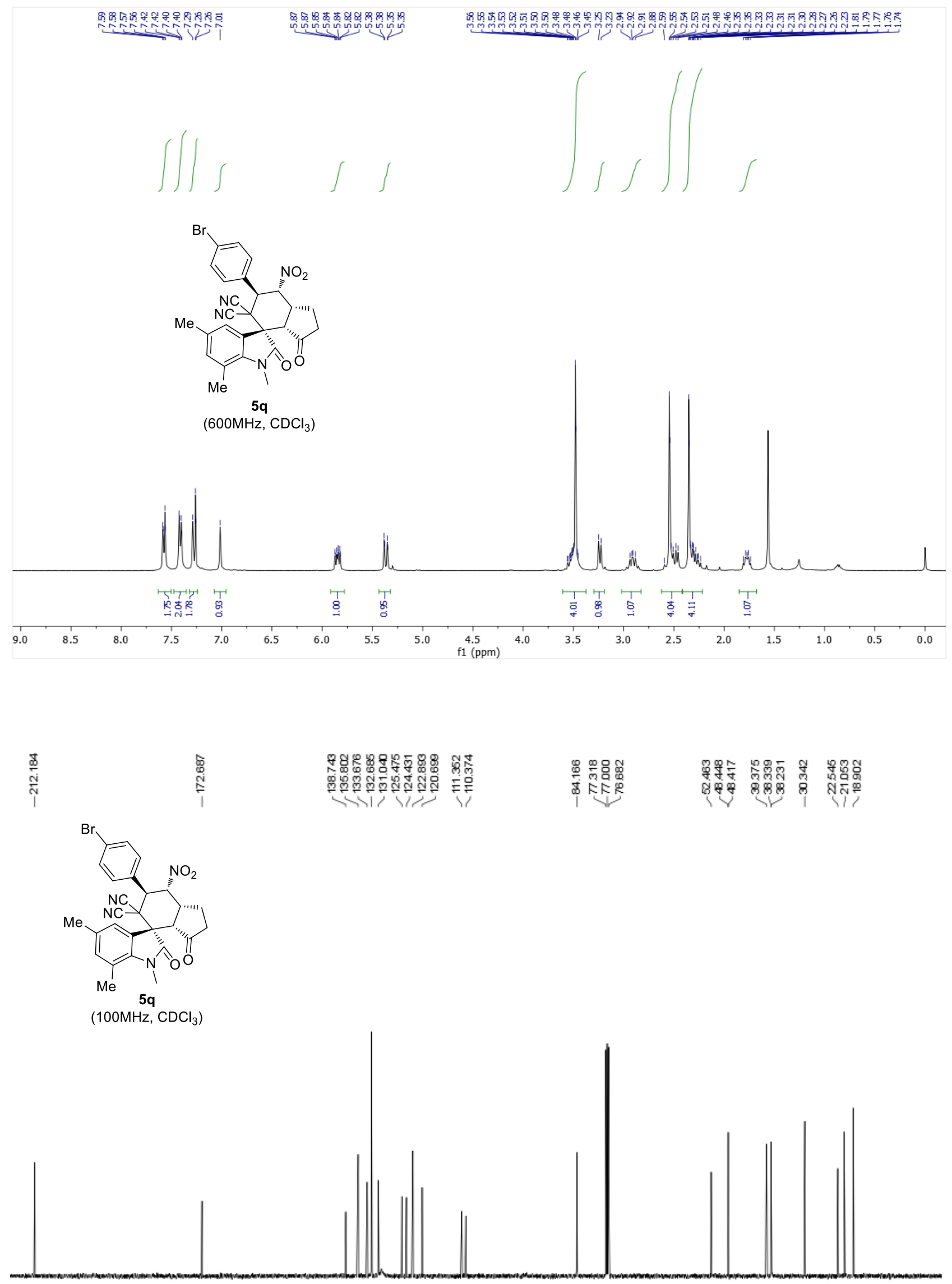

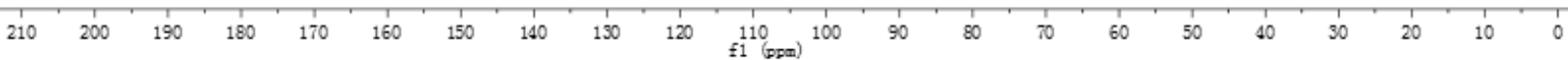




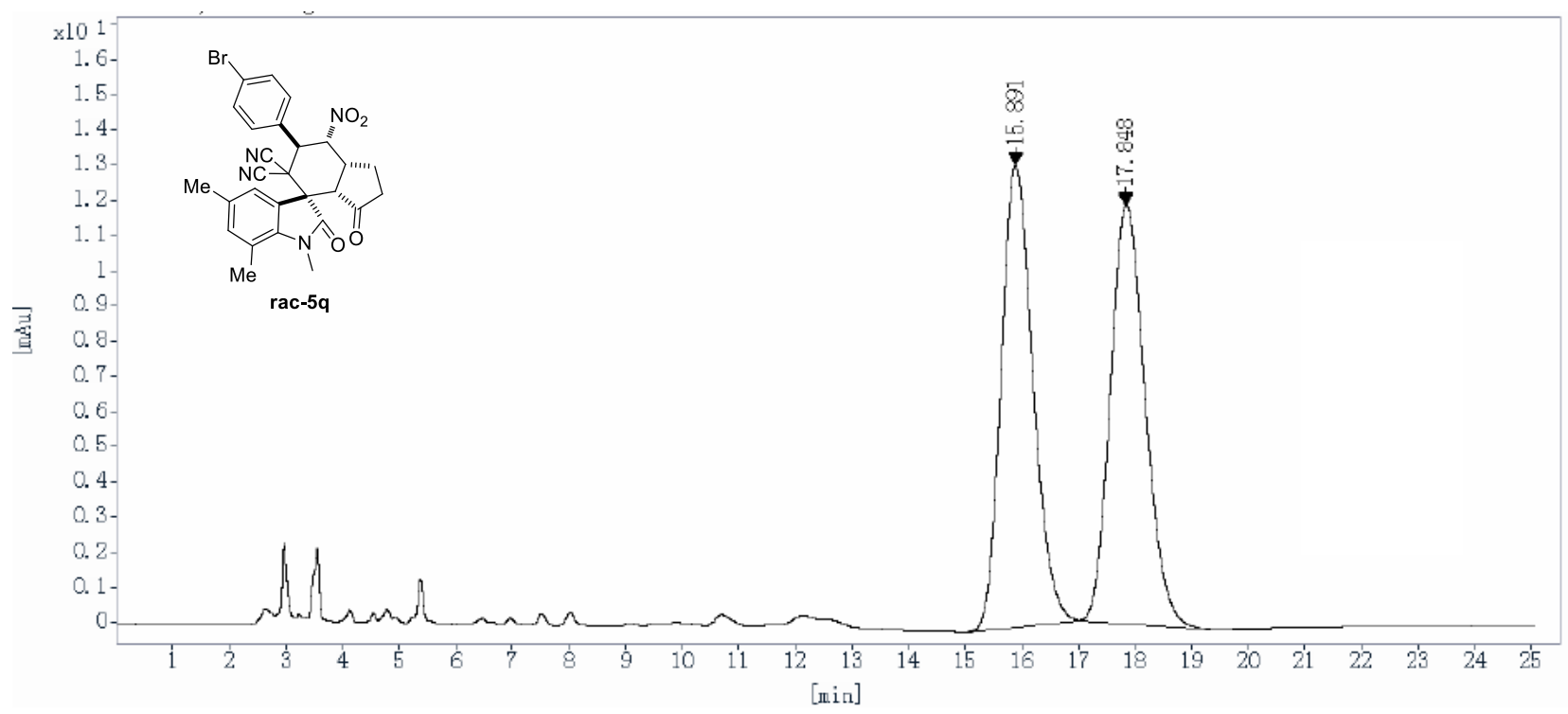

$\begin{array}{cccccc}\begin{array}{c}\text { Ret Time } \\ \text { [min] }\end{array} & \begin{array}{c}\text { Peak } \\ \text { Type }\end{array} & \begin{array}{c}\text { Width } \\ {[\mathrm{min}]}\end{array} & \begin{array}{c}\text { Height } \\ {[\mathrm{mAU}]}\end{array} & \begin{array}{c}\text { Area } \\ {\left[\mathbf{m A U}^{*} \mathrm{~s}\right]}\end{array} & \begin{array}{c}\text { Area } \\ {[\%]}\end{array} \\ 15.891 & \text { BB } & 0.60 & 13.1100 & 510.7031 & 50.1822 \\ 17.848 & \text { BB } & 0.66 & 11.8919 & 506.9955 & 49.8178\end{array}$

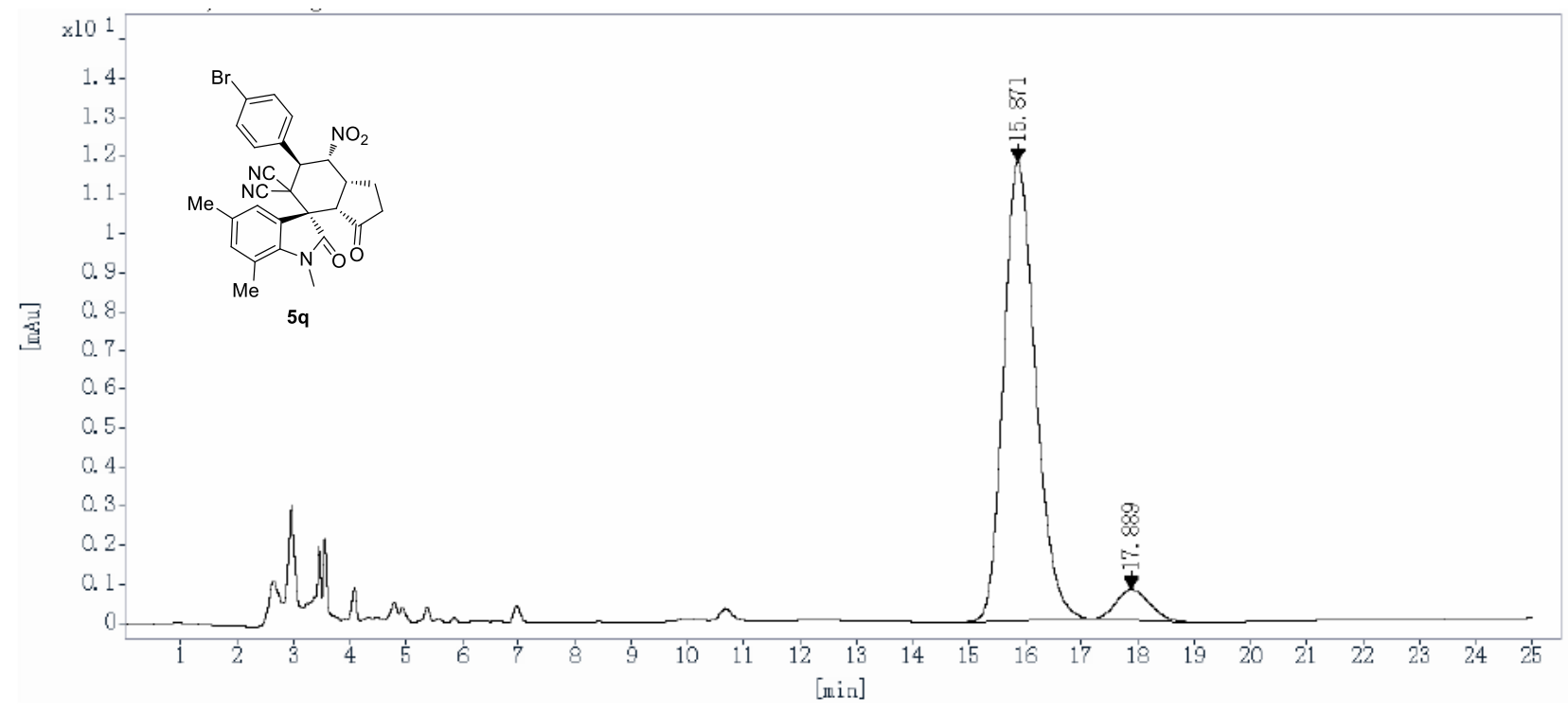

\begin{tabular}{cccccc}
$\begin{array}{c}\text { Ret Time } \\
\text { [min] }\end{array}$ & $\begin{array}{c}\text { Peak } \\
\text { Type }\end{array}$ & $\begin{array}{c}\text { Width } \\
{[\mathrm{min}]}\end{array}$ & $\begin{array}{c}\text { Height } \\
{[\mathrm{mAU}]}\end{array}$ & $\begin{array}{c}\text { Area } \\
{\left[\mathrm{mAU}^{*} \mathrm{~s}\right]}\end{array}$ & $\begin{array}{c}\text { Area } \\
{[\%]}\end{array}$ \\
\hline 15.871 & BB & 0.60 & 11.7712 & 463.4202 & 93.5805 \\
17.889 & BB & 0.50 & 0.7778 & 31.7898 & 6.4195
\end{tabular}




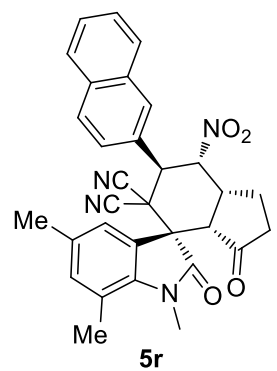

$\left(600 \mathrm{MHz}, \mathrm{CDCl}_{3}\right)$

$\stackrel{\text { กี }}{\text { ก }}$

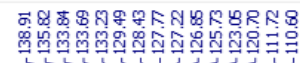

草

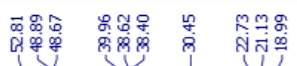<smiles>Cc1cc(C)c2c(c1)[C@@]1(C(=O)N(Br)c3ccccc3)[C@H]3C(=O)CC[C@H]3[C@@H]([N+](=O)[O-])[C@H](c3ccc4ccccc4c3)[C@@]21C#N</smiles>

$\left(150 \mathrm{MHz}, \mathrm{CDCl}_{3}\right)$

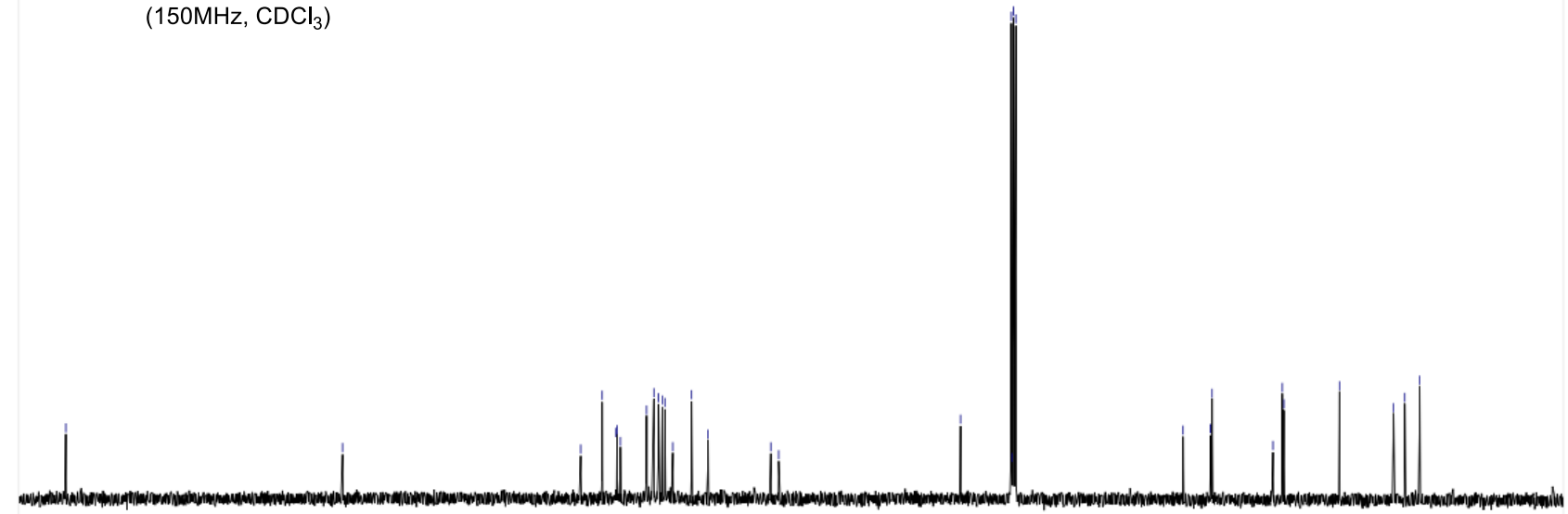

$\begin{array}{lllllllllll}210 & 200 & 190 & 180 & 170 & 160 & 150 & 140 & 130 & 120 & \underset{\mathrm{f} 1(\mathrm{ppm})}{110}\end{array}$ 


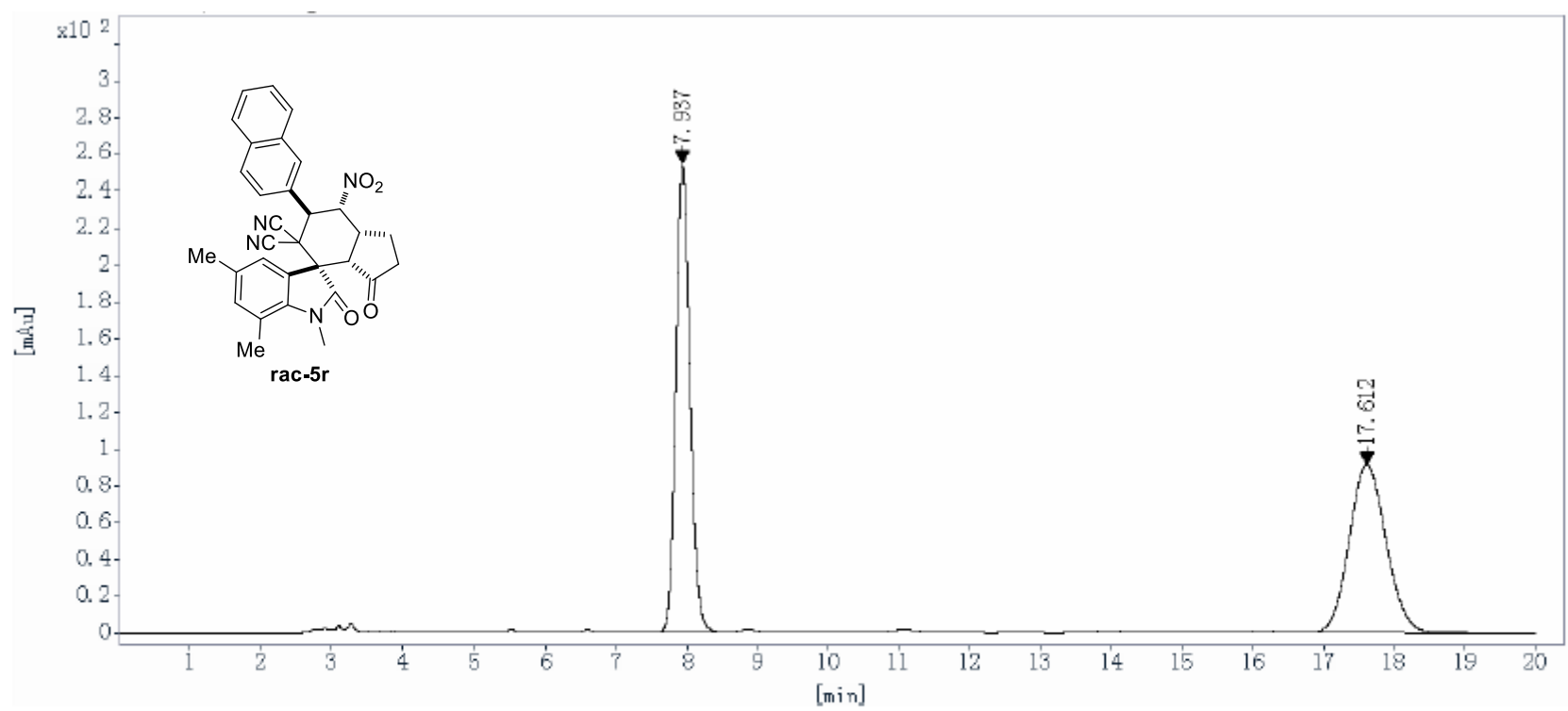

$\begin{array}{cccccc}\begin{array}{c}\text { Ret Time } \\ \text { [min] }\end{array} & \begin{array}{c}\text { Peak } \\ \text { Type }\end{array} & \begin{array}{c}\text { Width } \\ {[\mathrm{min}]}\end{array} & \begin{array}{c}\text { Height } \\ {[\mathrm{mAU}]}\end{array} & \begin{array}{c}\text { Area } \\ {\left[\mathbf{m A U}^{*} \text { s] }\right.}\end{array} & \begin{array}{c}\text { Area } \\ {[\%]}\end{array} \\ 7.937 & \text { BB } & 0.22 & 254.2308 & 3553.9900 & 51.6617 \\ 17.612 & \text { BBA } & 0.57 & 91.3677 & 3325.3596 & 48.3383\end{array}$

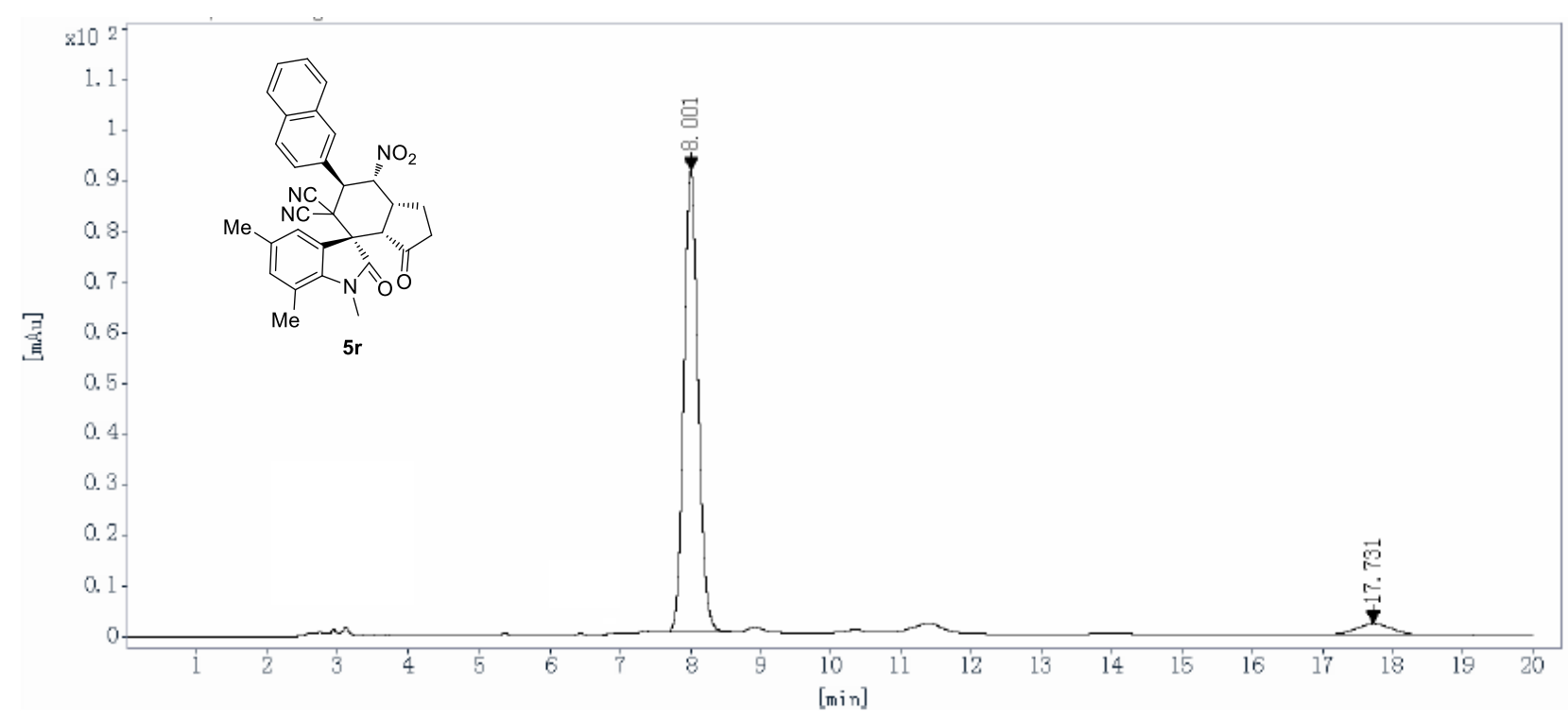

$\begin{array}{cccccc}\begin{array}{c}\text { Ret Time } \\ \text { [min] }\end{array} & \begin{array}{c}\text { Peak } \\ \text { Type }\end{array} & \begin{array}{c}\text { Width } \\ {[\mathrm{min}]}\end{array} & \begin{array}{c}\text { Height } \\ {[\mathrm{mAU}]}\end{array} & \begin{array}{c}\text { Area } \\ {\left[\mathrm{mAU} \mathbf{A U}^{*} \mathbf{c}\right]}\end{array} & \begin{array}{c}\text { Area } \\ {[\%]}\end{array} \\ 8.001 & \text { BB } & 0.22 & 91.0733 & 1299.8302 & 94.2841 \\ 17.731 & \text { BB } & 0.54 & 2.1994 & 78.8008 & 5.7159\end{array}$




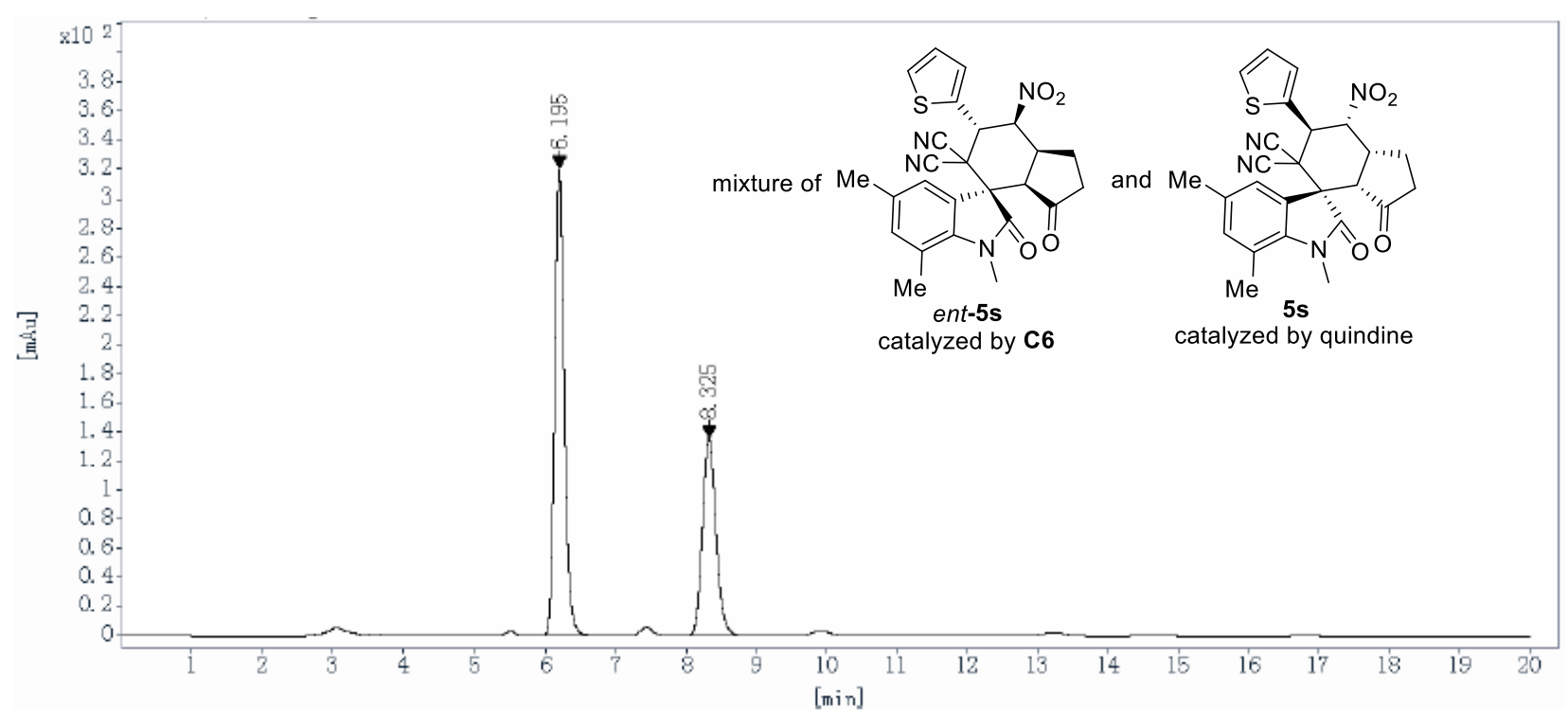

\begin{tabular}{cccccc}
$\begin{array}{c}\text { Ret Time } \\
{[\mathrm{min}]}\end{array}$ & $\begin{array}{c}\text { Peak } \\
\text { Type }\end{array}$ & $\begin{array}{c}\text { Wilth } \\
{[\mathrm{min}]}\end{array}$ & $\begin{array}{c}\text { Height } \\
{[\mathrm{mAU}]}\end{array}$ & $\begin{array}{c}\text { Area } \\
{\left[\mathrm{mAU}^{*} \text { s] }\right.}\end{array}$ & $\begin{array}{c}\text { Area } \\
{[\%]}\end{array}$ \\
\hline 6.195 & BB & 0.15 & 319.6223 & 3068.2593 & 62.6669 \\
8.325 & BB & 0.21 & 135.8993 & 1827.8781 & 37.3331
\end{tabular}

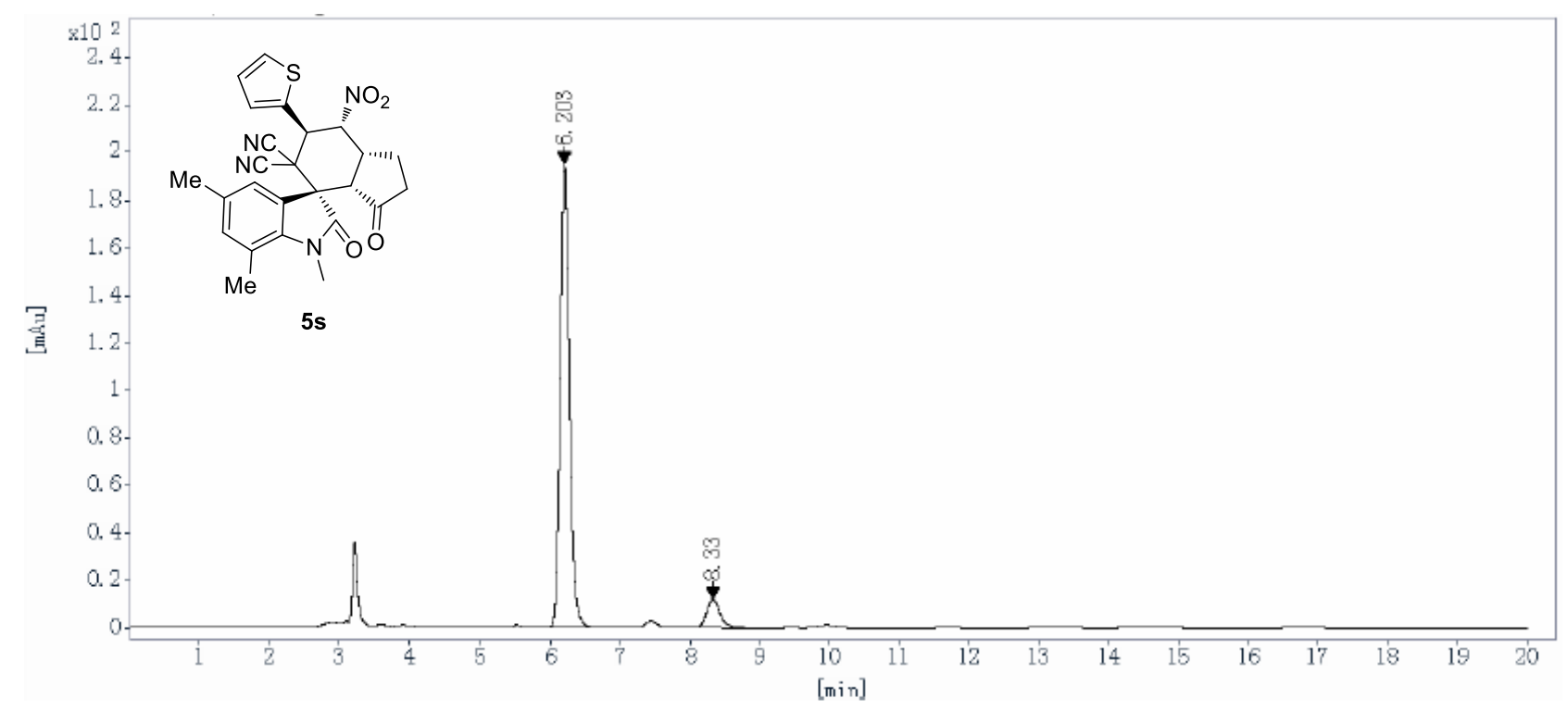

$\begin{array}{cccccc}\begin{array}{c}\text { Ret Time } \\ {[\mathrm{min}]}\end{array} & \begin{array}{c}\text { Peak } \\ \text { Type }\end{array} & \begin{array}{c}\text { Width } \\ {[\mathrm{min}]}\end{array} & \begin{array}{c}\text { Height } \\ {[\mathrm{mAU}]}\end{array} & \begin{array}{c}\text { Area } \\ {\left[\mathrm{mAU}^{*} \mathrm{~s}\right]}\end{array} & \begin{array}{c}\text { Area } \\ {[\%]}\end{array} \\ 6.203 & \text { BBA } & 0.14 & 194.7155 & 1813.7520 & 92.0495 \\ 8.330 & \text { BB } & 0.21 & 11.6859 & 156.6579 & 7.9505\end{array}$



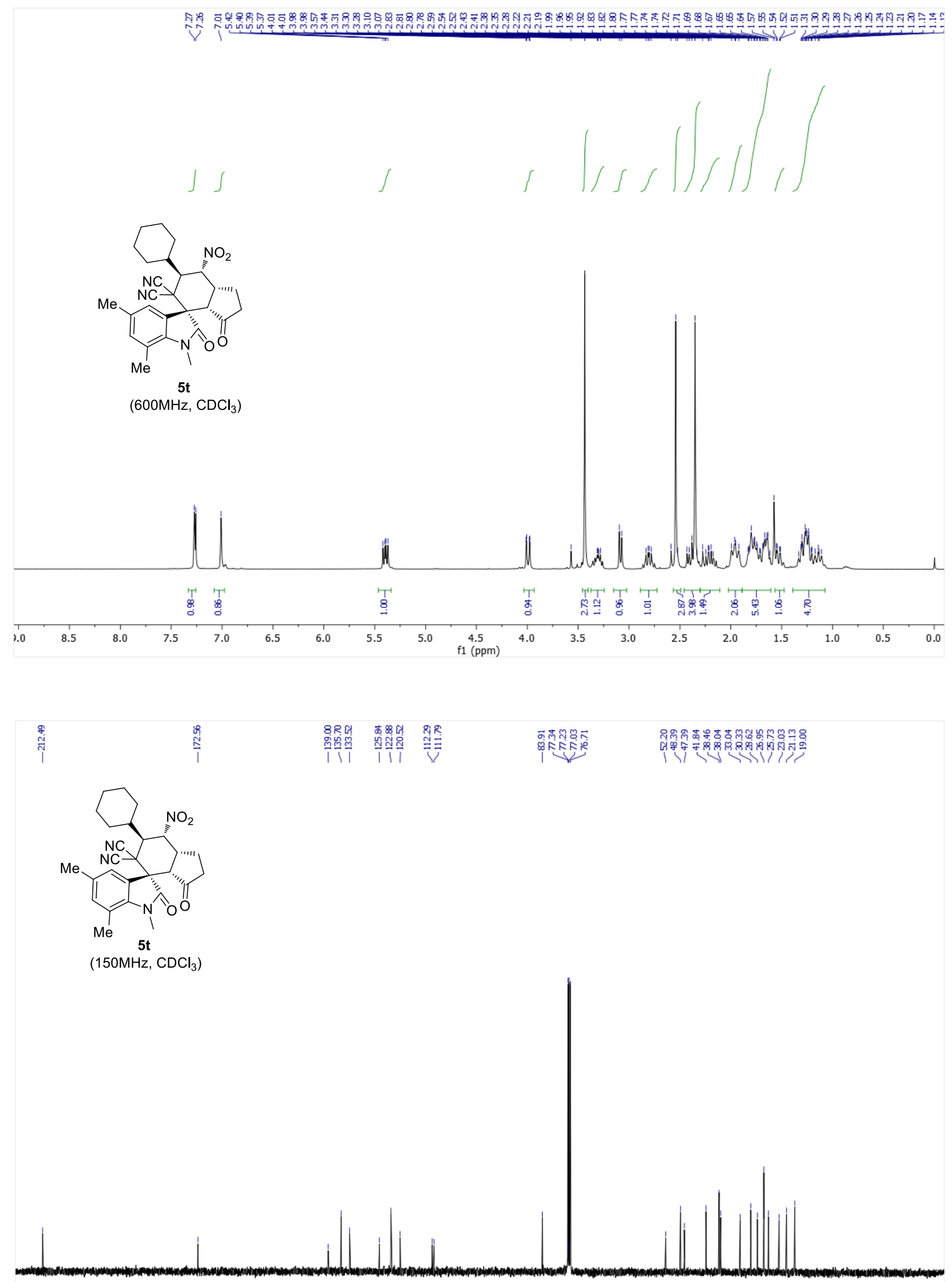

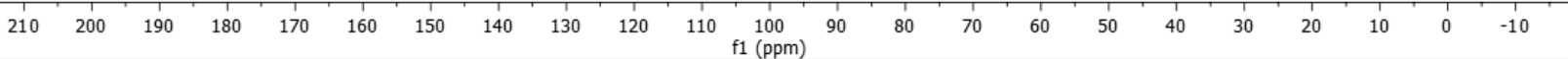




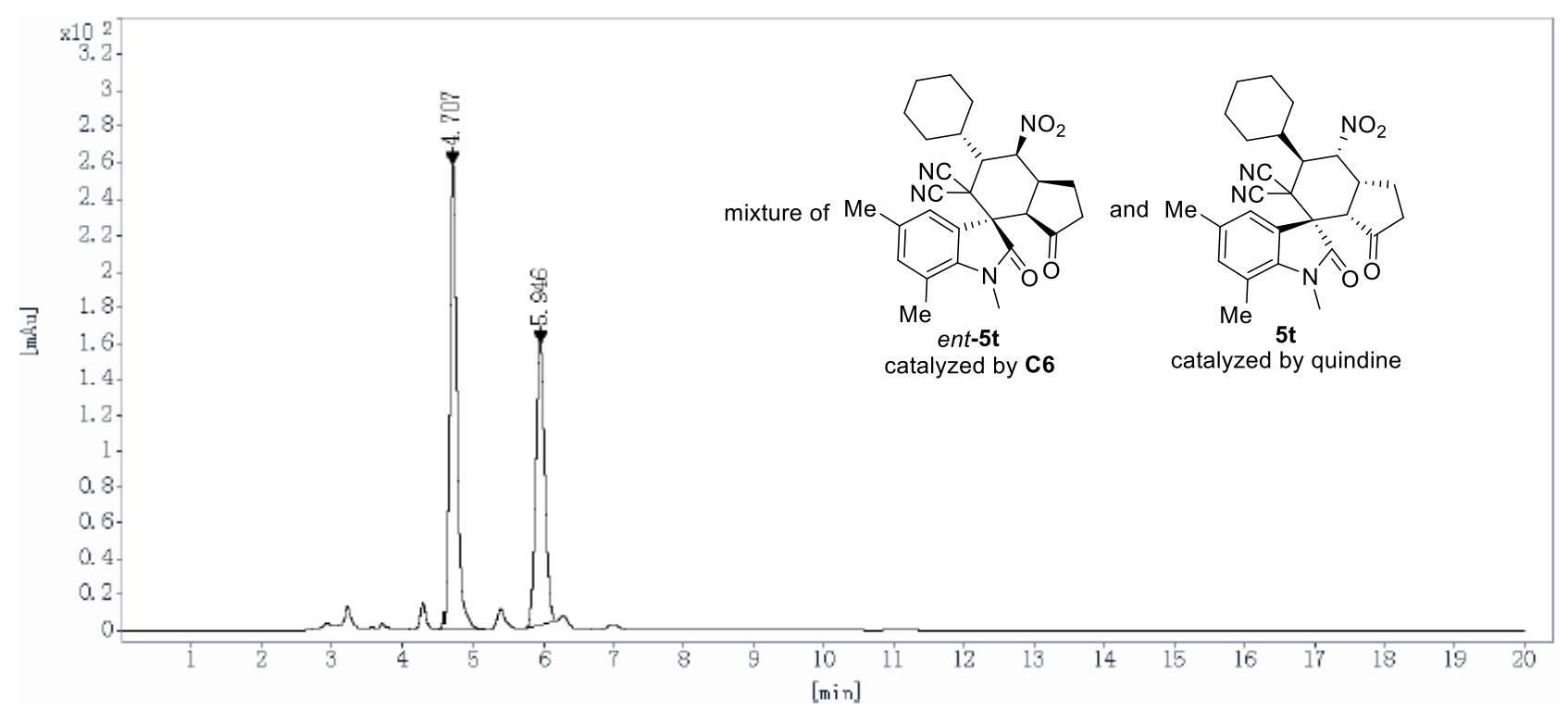

\begin{tabular}{cccccc}
$\begin{array}{c}\text { Ret Time } \\
{[\mathrm{min}]}\end{array}$ & $\begin{array}{c}\text { Peak } \\
\text { Type }\end{array}$ & $\begin{array}{c}\text { Width } \\
{[\mathrm{min}]}\end{array}$ & $\begin{array}{c}\text { Height } \\
{[\mathrm{mAU}]}\end{array}$ & $\begin{array}{c}\text { Area } \\
{\left[\mathrm{mAU}^{*} \text { s] }\right.}\end{array}$ & $\begin{array}{c}\text { Area } \\
{[\%]}\end{array}$ \\
\hline 4.707 & BB & 0.11 & 257.7388 & 1850.9292 & 56.9245 \\
5.946 & BB & 0.14 & 156.9358 & 1400.6216 & 43.0755
\end{tabular}

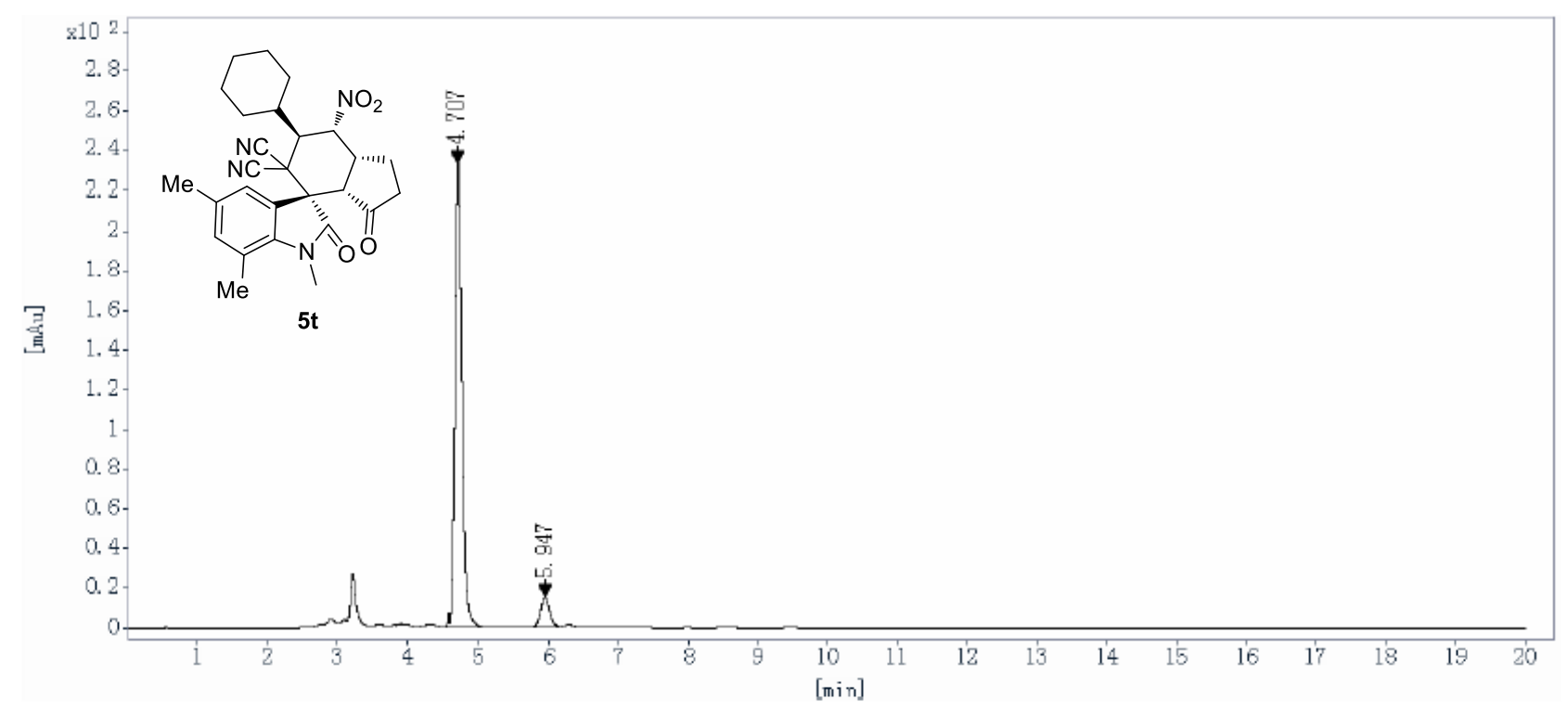

\begin{tabular}{cccccc}
$\begin{array}{c}\text { Ret Time } \\
\text { [min] }\end{array}$ & $\begin{array}{c}\text { Peak } \\
\text { Type }\end{array}$ & $\begin{array}{c}\text { Width } \\
{[\mathrm{min}]}\end{array}$ & $\begin{array}{c}\text { Height } \\
{[\mathrm{mAU}]}\end{array}$ & $\begin{array}{c}\text { Area } \\
{\left[\mathrm{mAU}^{*} \mathbf{s}\right]}\end{array}$ & $\begin{array}{c}\text { Area } \\
{[\%]}\end{array}$ \\
\hline 4.707 & BB & 0.11 & 232.7814 & 1625.1051 & 92.5136 \\
5.947 & BB & 0.14 & 14.8609 & 131.5076 & 7.4864
\end{tabular}




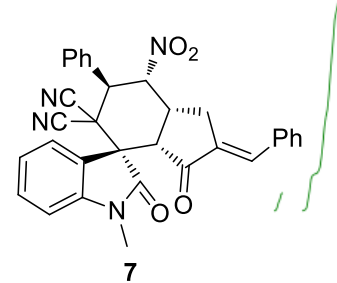

(600MHz, $\mathrm{CDCl}_{3}$ )
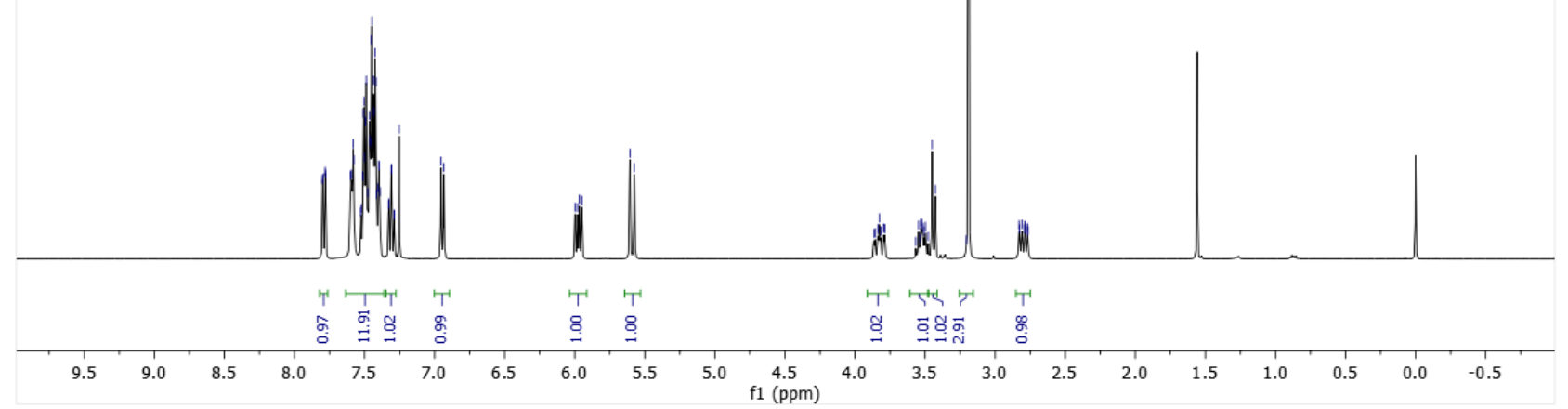

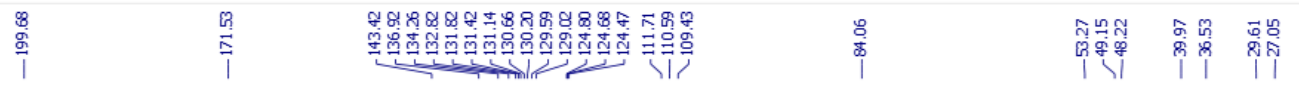

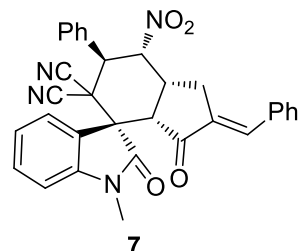

$\left(150 \mathrm{MHz}, \mathrm{CDCl}_{3}\right)$

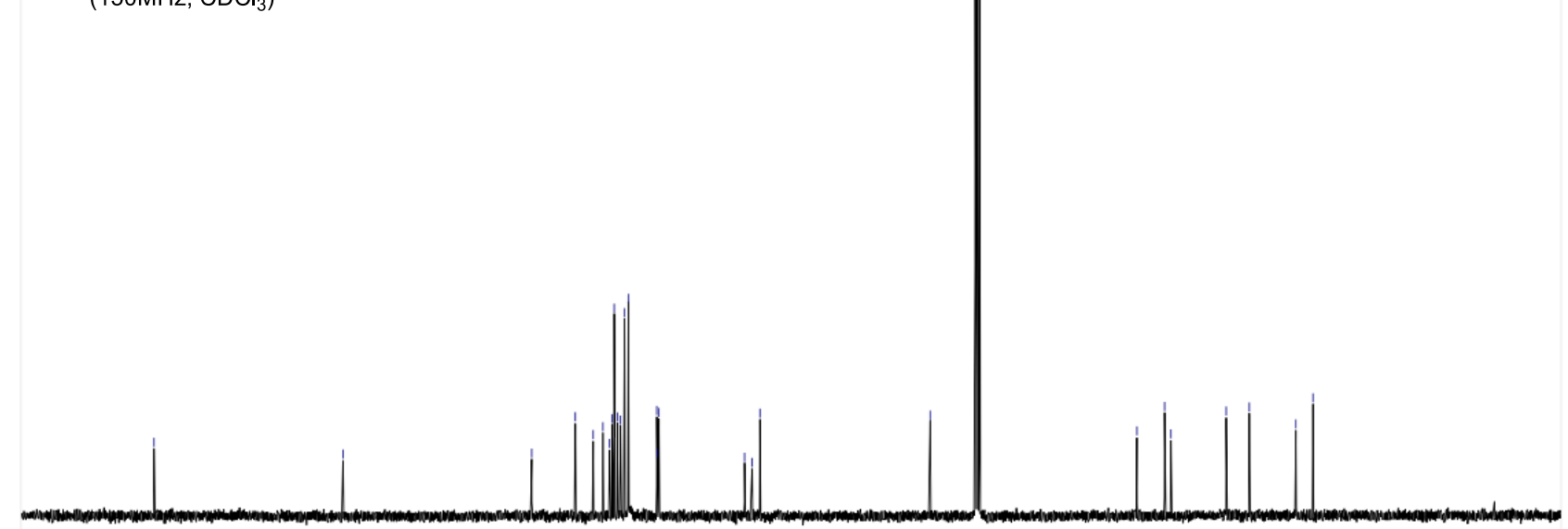

$\begin{array}{rrrrrrrrrrrr}210 & 200 & 190 & 180 & 170 & 160 & 150 & 140 & 130 & 120 & \begin{array}{c}110 \\ \mathrm{f} 1(\mathrm{ppm})\end{array}\end{array}$ 

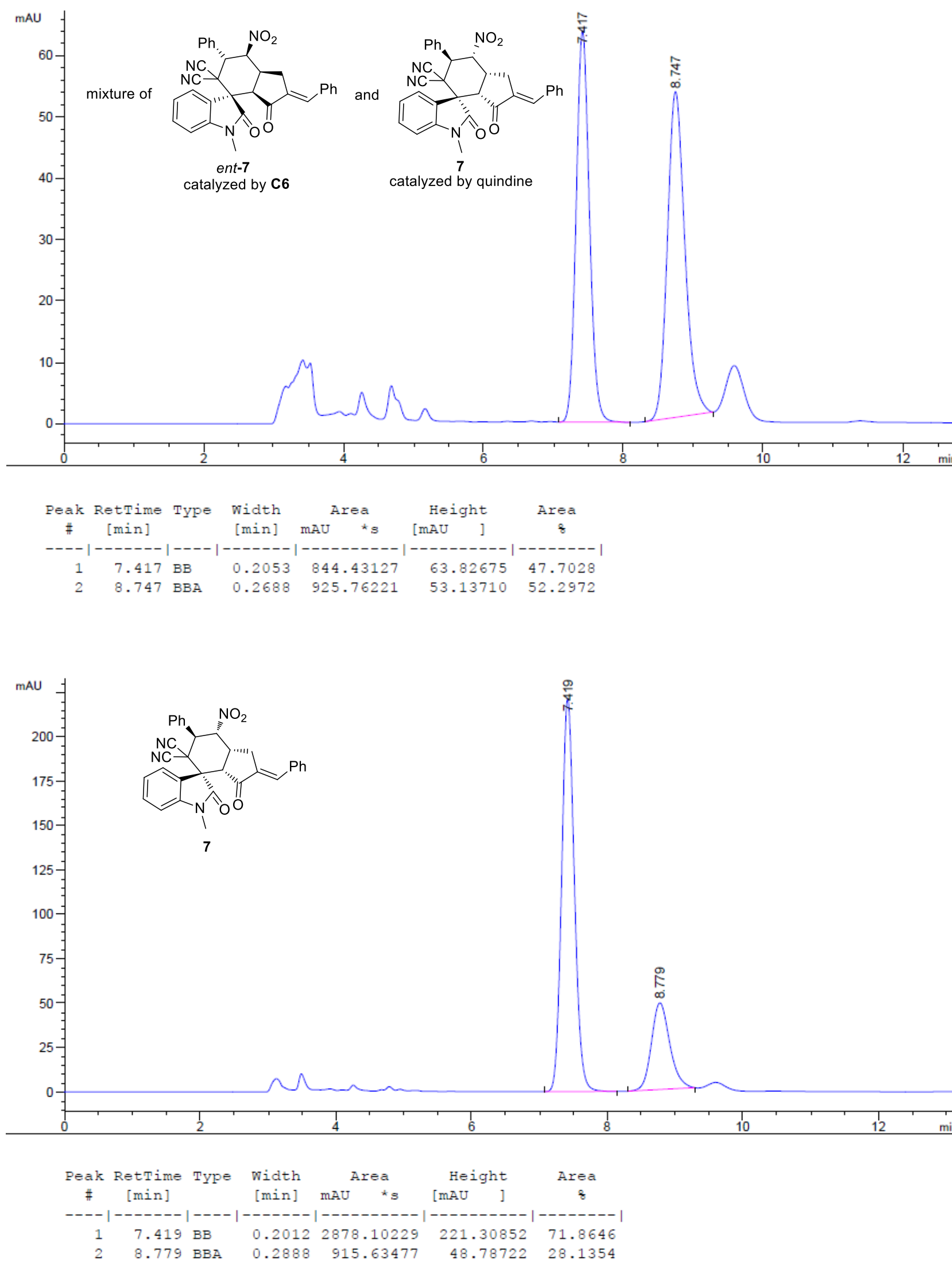

S98 


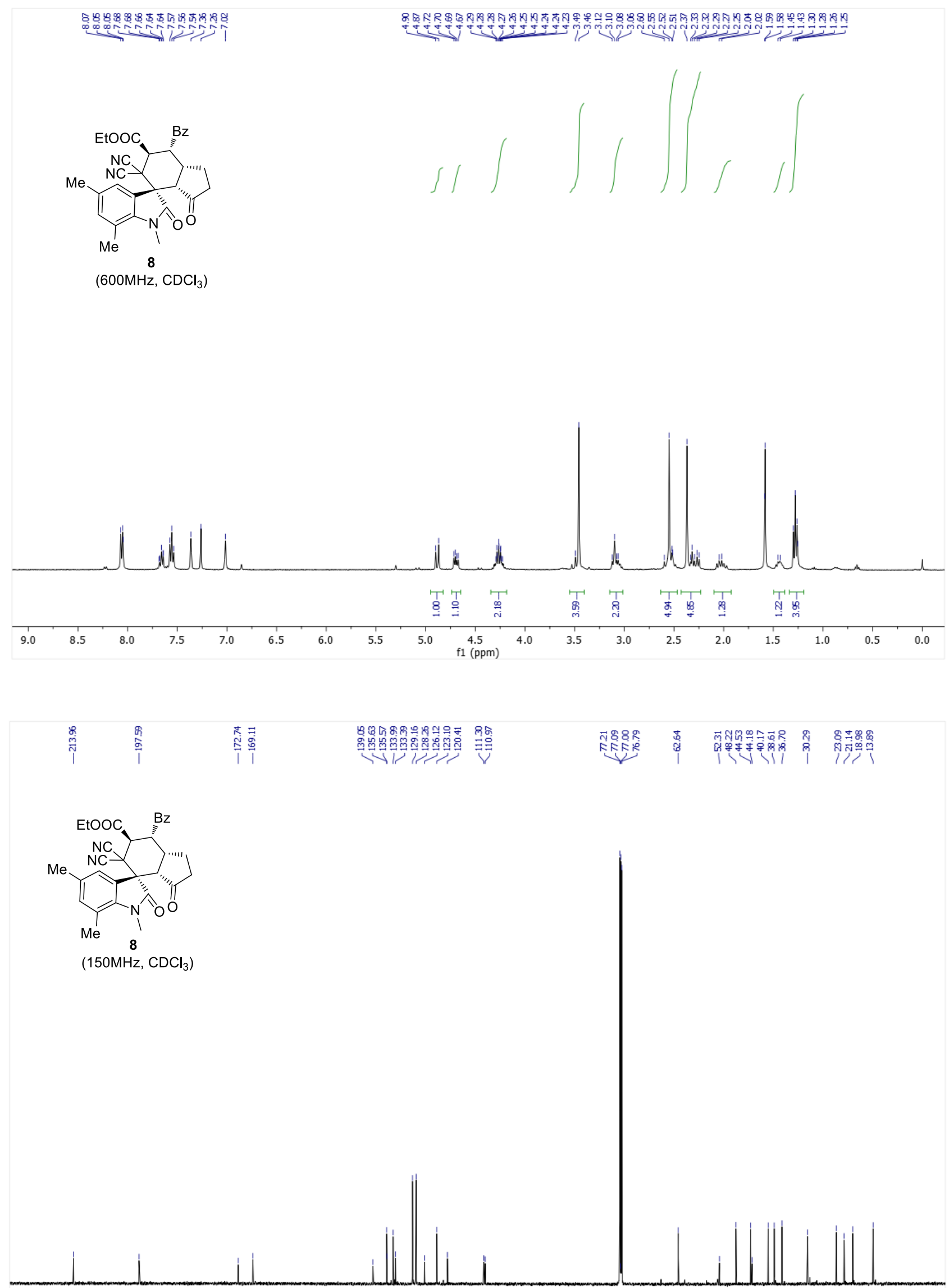

(150MHz, $\left.\mathrm{CDCl}_{3}\right)$ 


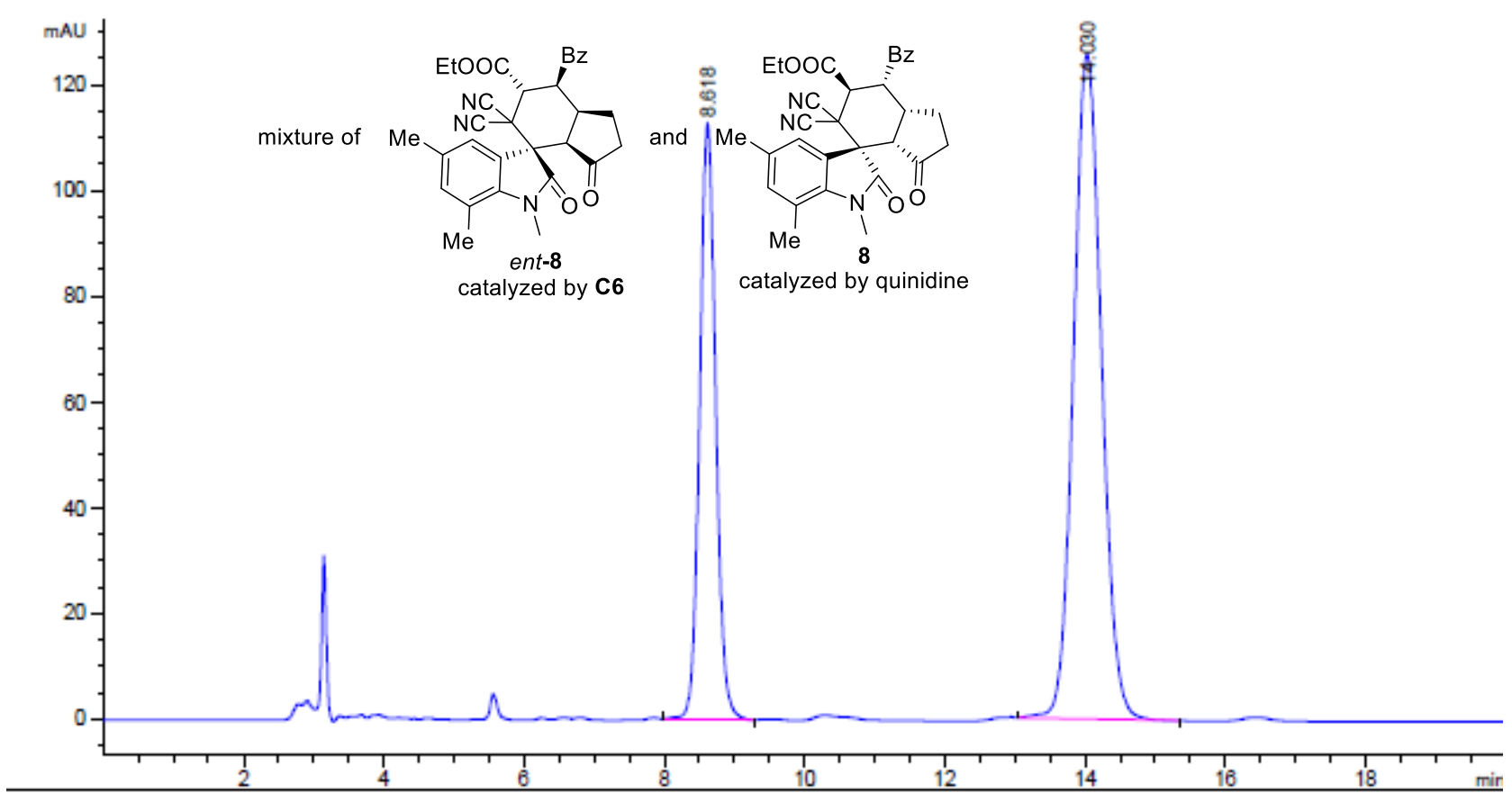

\begin{tabular}{rccccc}
$\begin{array}{c}\text { Ret Time } \\
\text { [min] }\end{array}$ & $\begin{array}{c}\text { Peak } \\
\text { Type }\end{array}$ & $\begin{array}{c}\text { Width } \\
\text { [min] }\end{array}$ & $\begin{array}{c}\text { Height } \\
\text { [mAU] }\end{array}$ & $\begin{array}{c}\text { Area } \\
{\left[\mathrm{mAU} \mathbf{H}^{*} \text { s] }\right.}\end{array}$ & $\begin{array}{c}\text { Area } \\
{[\%]}\end{array}$ \\
\hline 8.618 & BB & 0.2455 & 112.76895 & 1792.55115 & 33.8867 \\
14.030 & BB & 0.4322 & 125.86776 & 3497.28491 & 66.1133
\end{tabular}

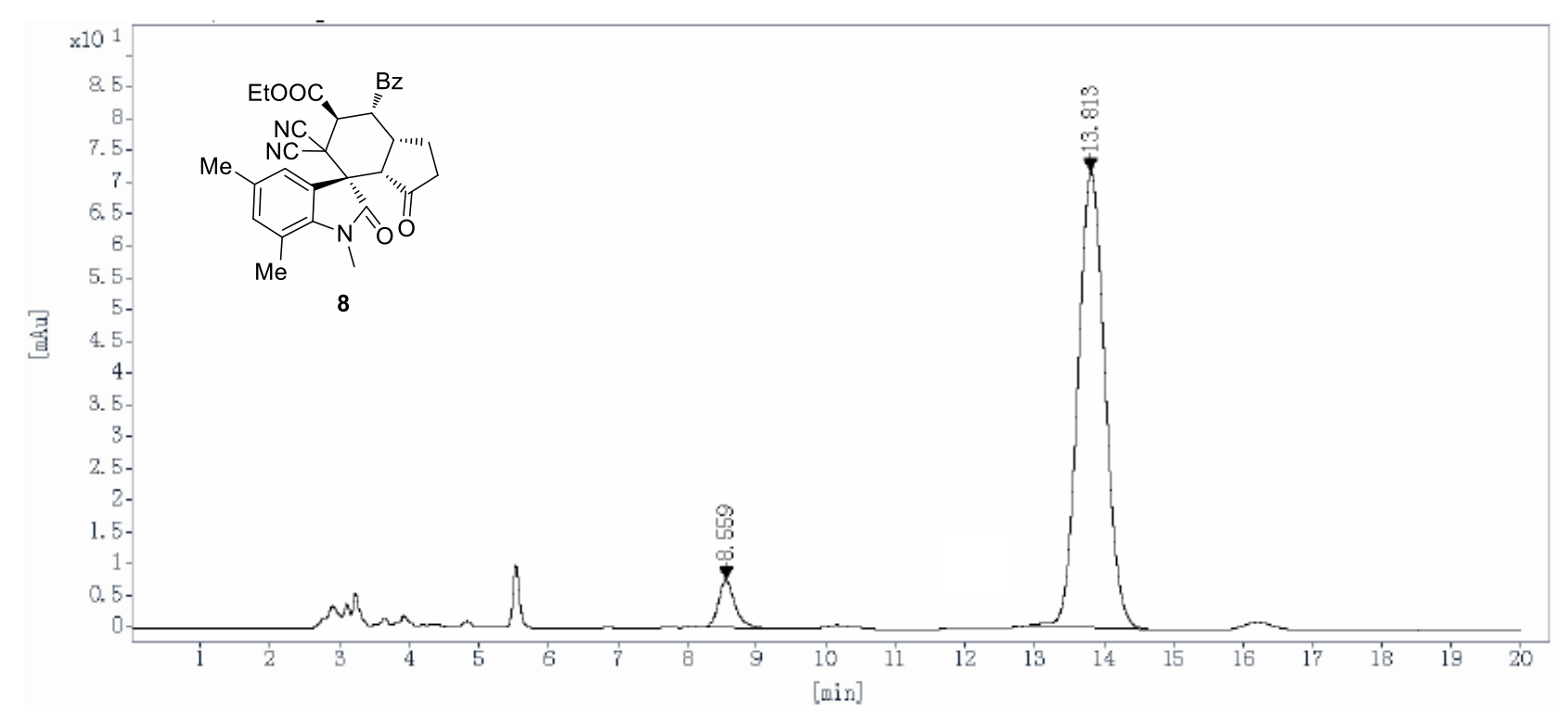

\begin{tabular}{cccccc}
$\begin{array}{c}\text { Ret Time } \\
\text { [min] }\end{array}$ & $\begin{array}{c}\text { Peak } \\
\text { Type }\end{array}$ & $\begin{array}{c}\text { Width } \\
{[\mathrm{min}]}\end{array}$ & $\begin{array}{c}\text { Height } \\
{[\mathrm{mAU}]}\end{array}$ & $\begin{array}{c}\text { Area } \\
{\left[\mathrm{mAU} \mathbf{A U}^{*} \text { ] }\right.}\end{array}$ & $\begin{array}{c}\text { Area } \\
{[\%]}\end{array}$ \\
\hline 8.559 & BB & 0.25 & 7.5587 & 123.9443 & 6.0385 \\
13.813 & BB & 0.42 & 71.6338 & 1928.6392 & 93.9615
\end{tabular}




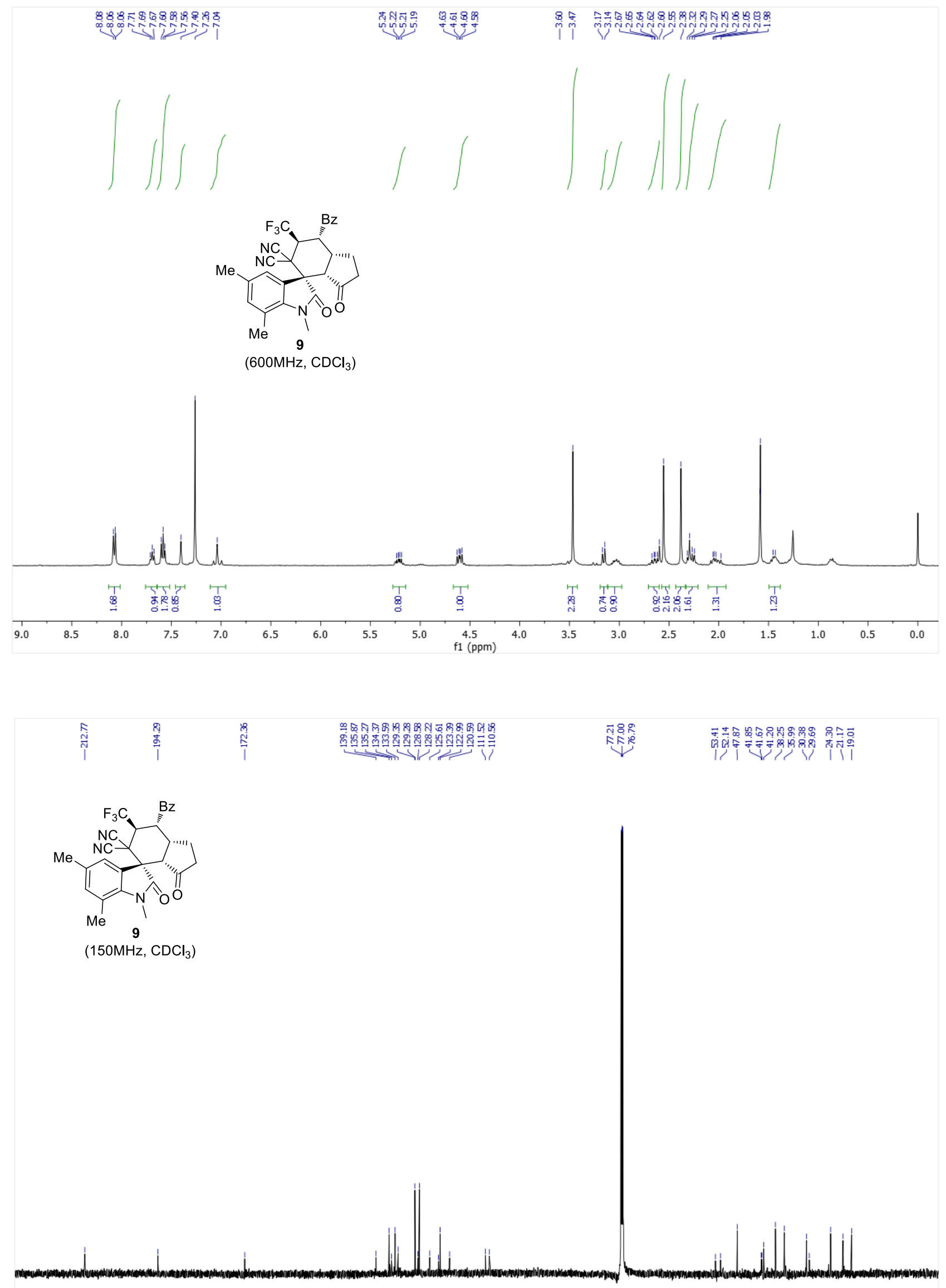

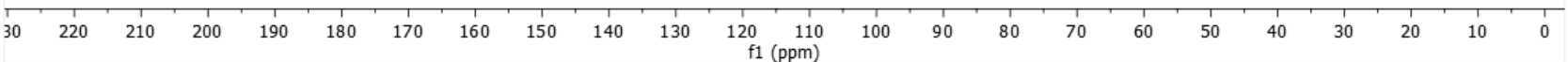



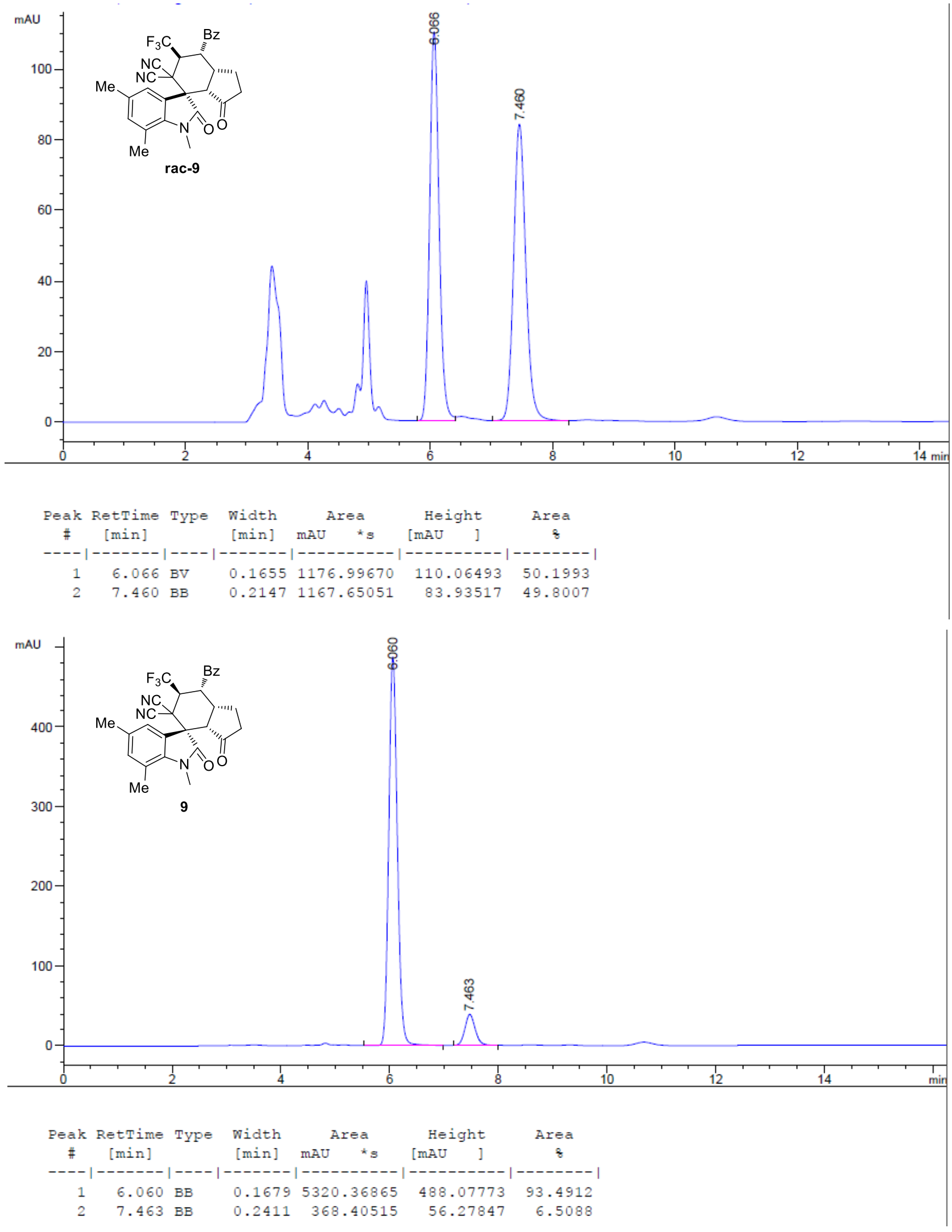


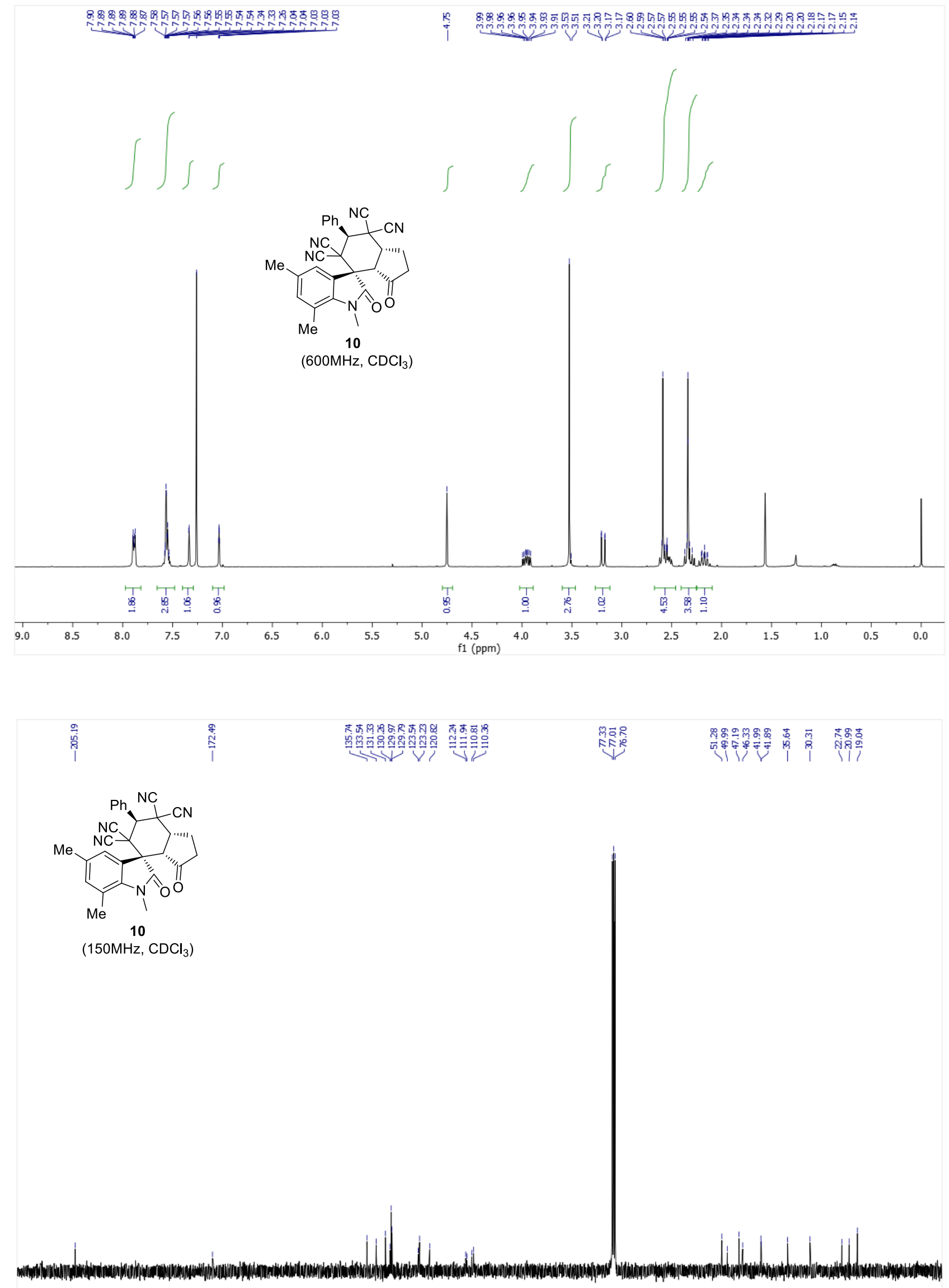

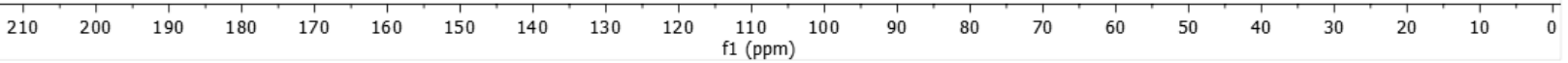



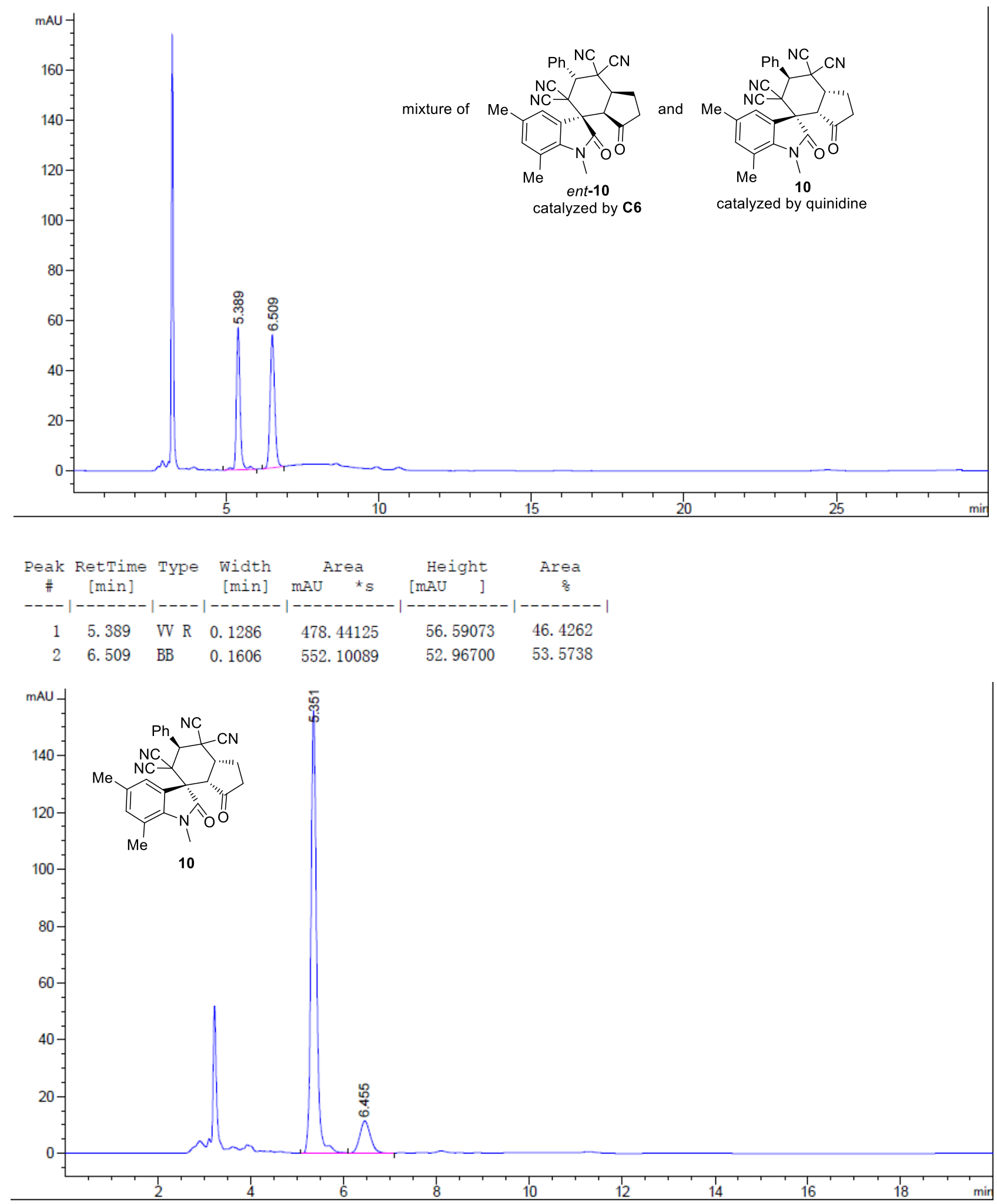

\begin{tabular}{|c|c|c|c|c|c|c|}
\hline \multirow{2}{*}{$\begin{array}{c}\text { Peak } \\
\#\end{array}$} & \multirow{2}{*}{$\begin{array}{c}\text { RetTime } \\
\text { [min] }\end{array}$} & \multirow{2}{*}{ Type } & \multirow{2}{*}{$\begin{array}{c}\text { Width } \\
\text { [min] }\end{array}$} & Area & Height & \multirow{2}{*}{$\begin{array}{c}\text { Area } \\
\frac{9}{6}\end{array}$} \\
\hline & & & & $\mathrm{mAU}$ & {$[\mathrm{mAU}$} & \\
\hline & & & & & & -- \\
\hline 1 & 5. 351 & VV $R$ & 0.1240 & 1334.30347 & 155.62289 & 94.4163 \\
\hline 2 & 6. 455 & $\mathrm{BB}$ & 0.1609 & 78.94641 & 16. 62692 & 5.5837 \\
\hline
\end{tabular}




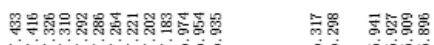

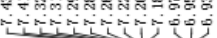

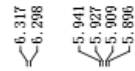

(1)

12a

$\left(400 \mathrm{MHz}, \mathrm{CDCl}_{3}\right)$

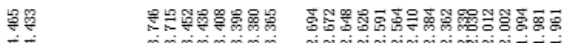

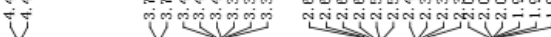

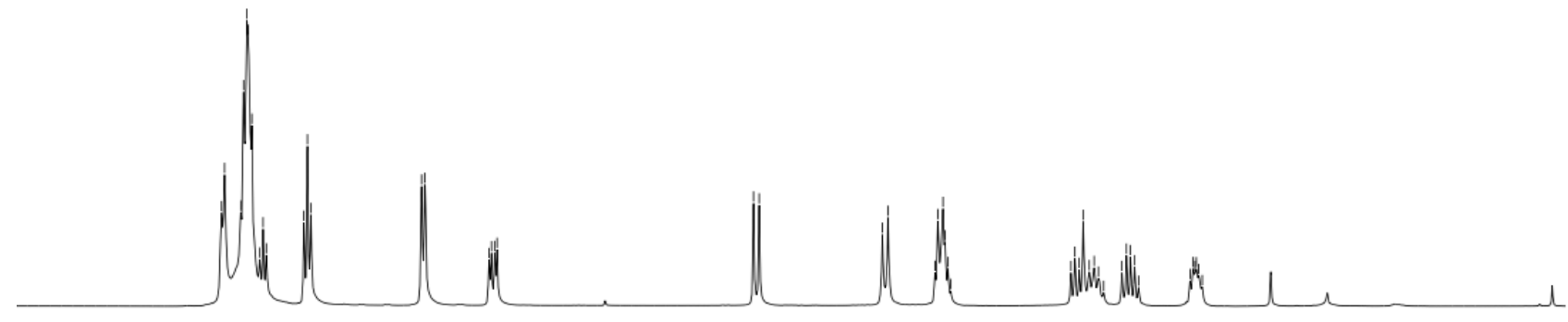

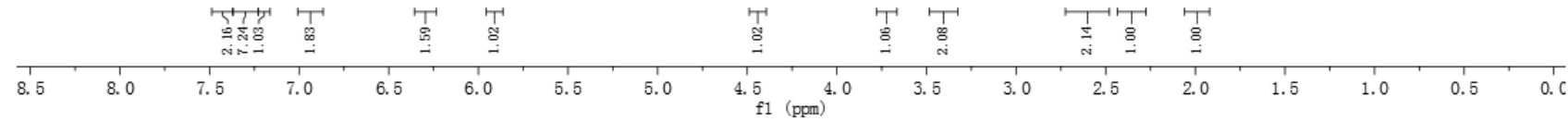

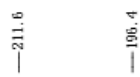

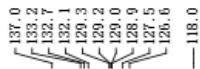

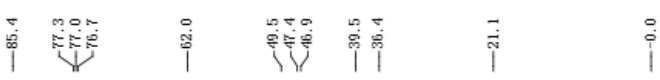

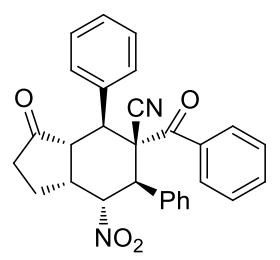

$12 \mathrm{a}$

$\left(100 \mathrm{MHz}, \mathrm{CDCl}_{3}\right)$

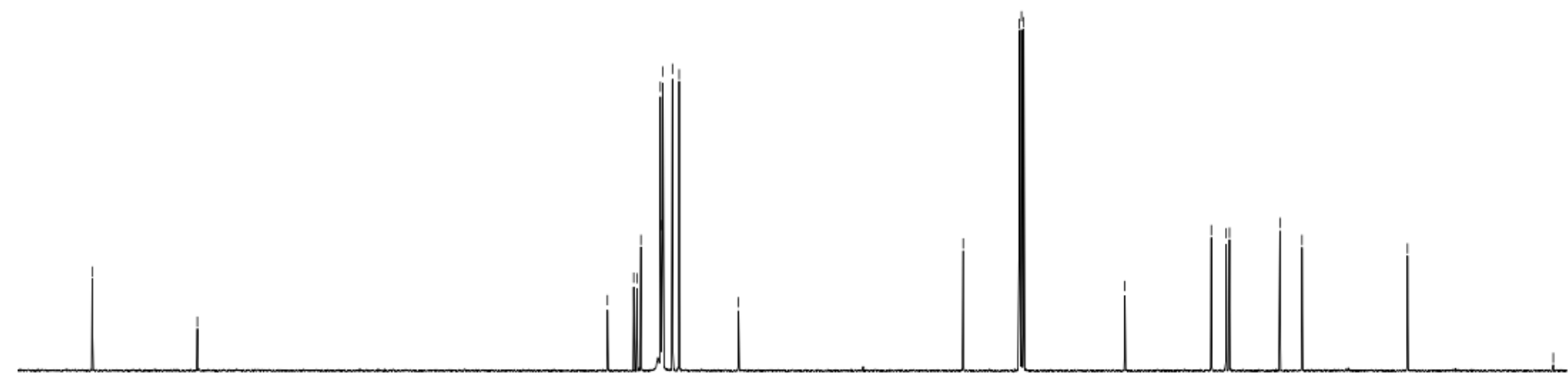

220

$\begin{array}{lllllllllll}210 & 200 & 190 & 180 & 170 & 160 & 150 & 140 & 130 & 120 & 110 \\ \mathrm{f} 1(\mathrm{ppm}) & 100\end{array}$ 


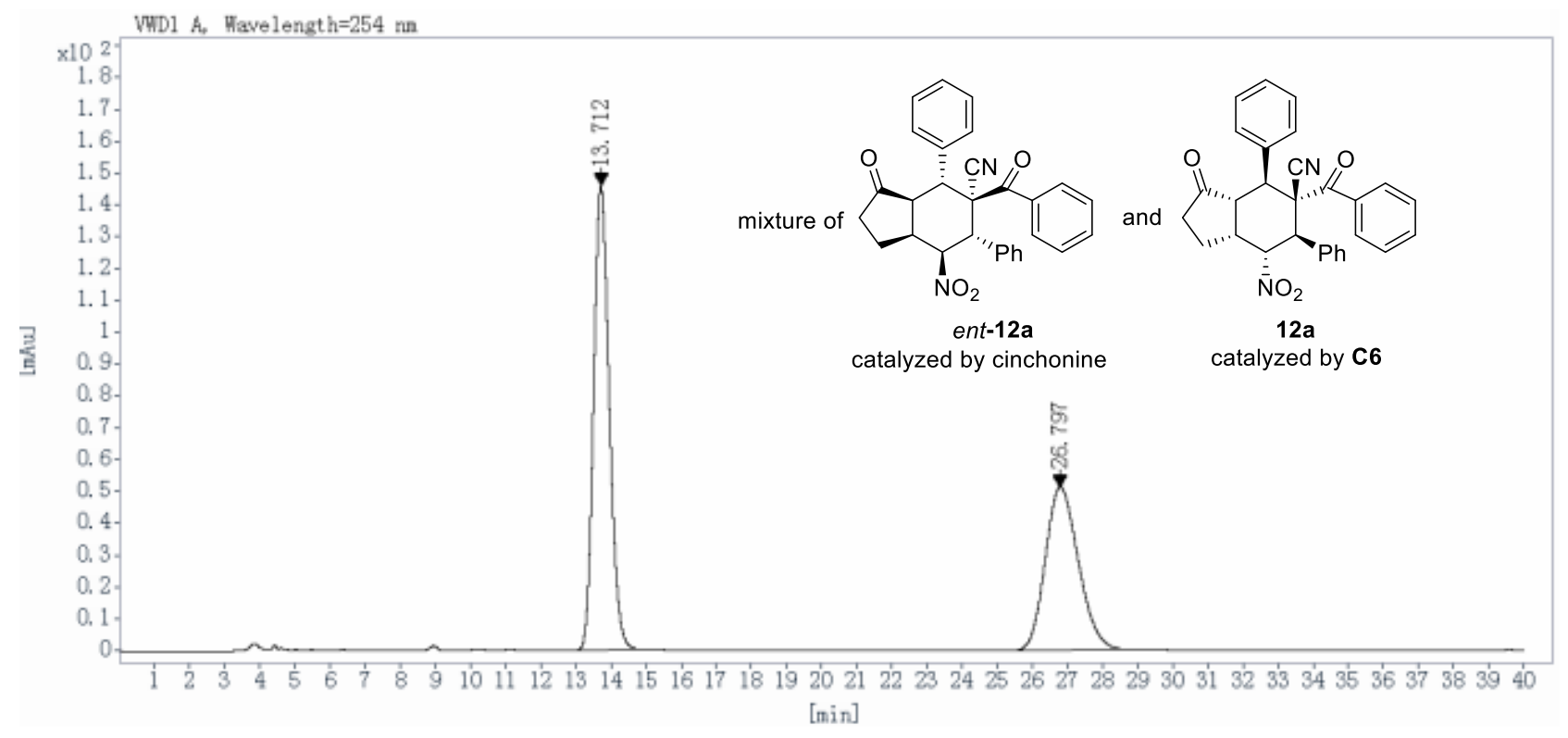

\begin{tabular}{cccccc}
$\begin{array}{c}\text { Ret Time } \\
\text { [min] }\end{array}$ & $\begin{array}{c}\text { Peak } \\
\text { Type }\end{array}$ & $\begin{array}{c}\text { Width } \\
{[\mathrm{min}]}\end{array}$ & $\begin{array}{c}\text { Height } \\
{[\mathrm{mAU}]}\end{array}$ & $\begin{array}{c}\text { Area } \\
{\left[\mathrm{mAU}^{*} \mathrm{~s}\right]}\end{array}$ & $\begin{array}{c}\text { Area } \\
{[\%]}\end{array}$ \\
\hline 13.712 & BB & 0.48 & 145.7370 & 4502.7183 & 57.1926 \\
26.797 & BB & 1.01 & 51.2258 & 3370.1804 & 42.8074 \\
& & & Totals: & 7872.8987 & 100.0000
\end{tabular}

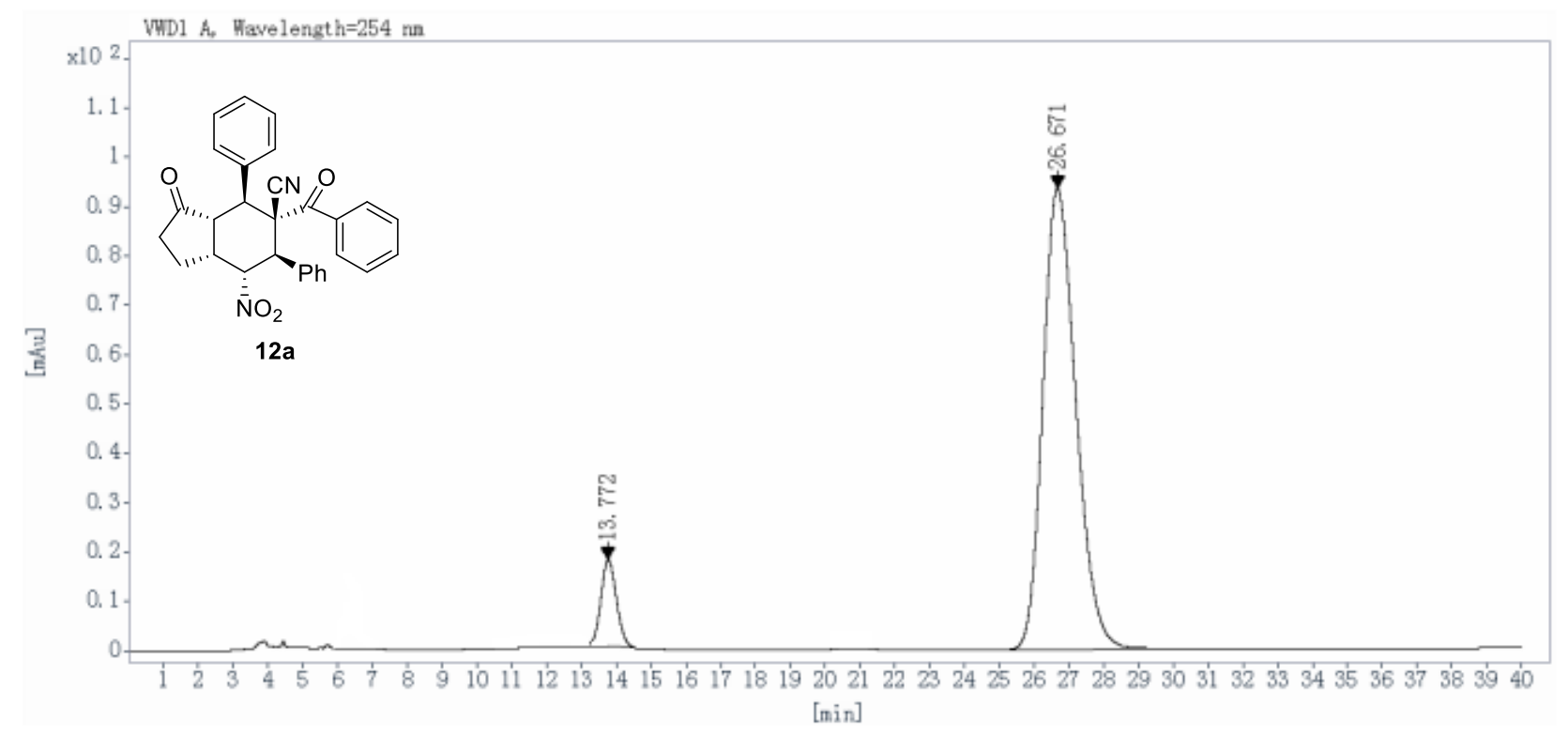

\begin{tabular}{cccccc}
$\begin{array}{c}\text { Ret Time } \\
\text { [min] }\end{array}$ & $\begin{array}{c}\text { Peak } \\
\text { Type }\end{array}$ & $\begin{array}{c}\text { Width } \\
{[\mathrm{min}]}\end{array}$ & $\begin{array}{c}\text { Height } \\
{[\mathrm{mAU}]}\end{array}$ & $\begin{array}{c}\text { Area } \\
{\left[\mathrm{mAU}^{*} \text { s] }\right.}\end{array}$ & $\begin{array}{c}\text { Area } \\
{[\%]}\end{array}$ \\
\hline 13.772 & BB & 0.48 & 17.4750 & 539.6851 & 8.0380 \\
26.671 & BB & 1.02 & $\begin{array}{c}93.4017 \\
\text { Totals: }\end{array}$ & 6174.4922 & 91.9620 \\
& & & 6714.1772 & 100.0000
\end{tabular}



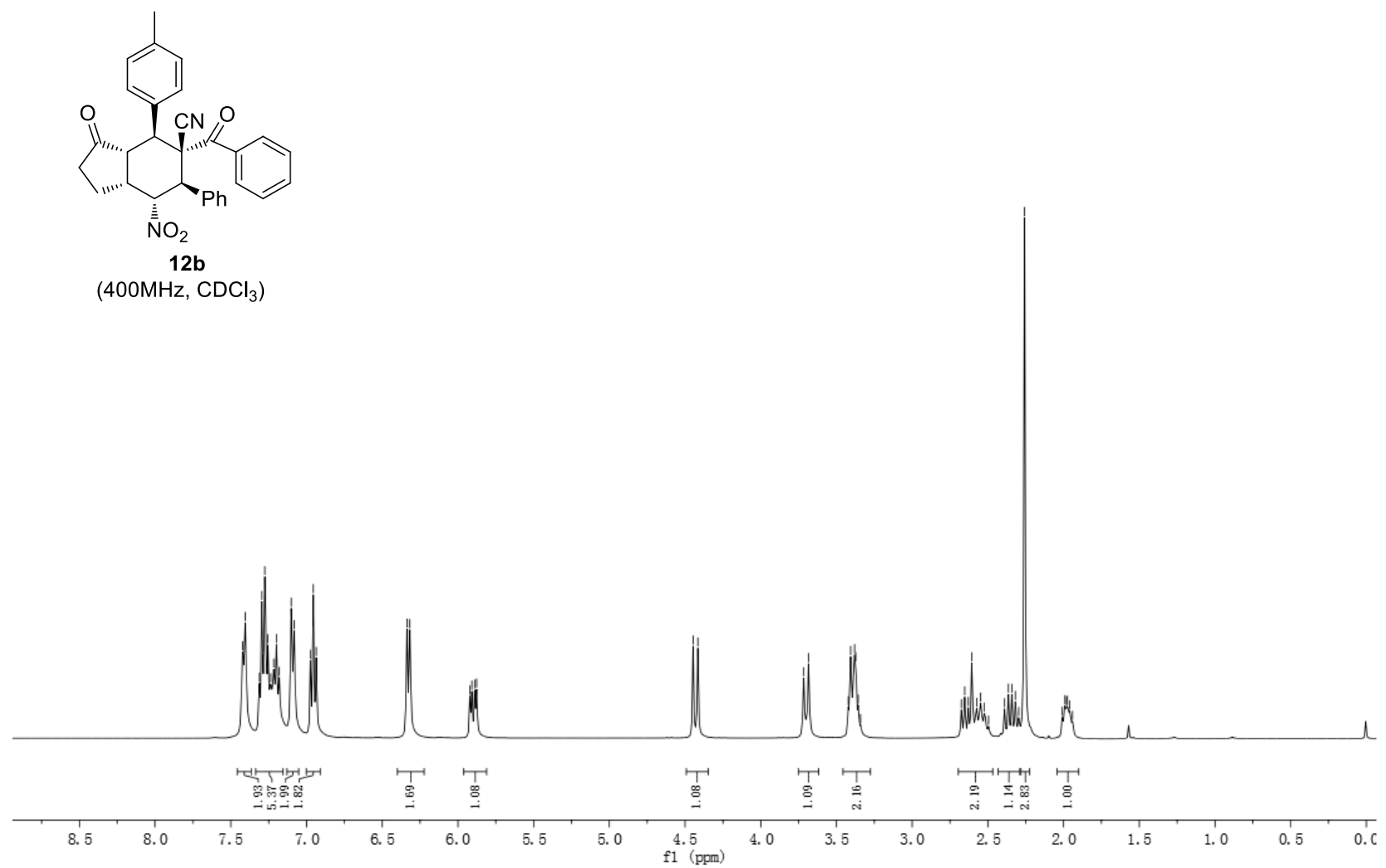

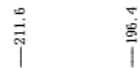

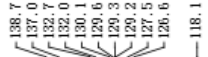

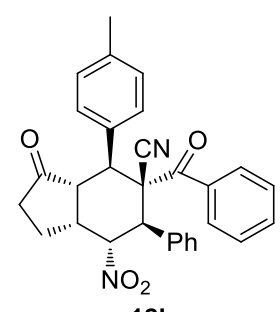

$\left(100 \mathrm{MHz}, \mathrm{CDCl}_{3}\right)$

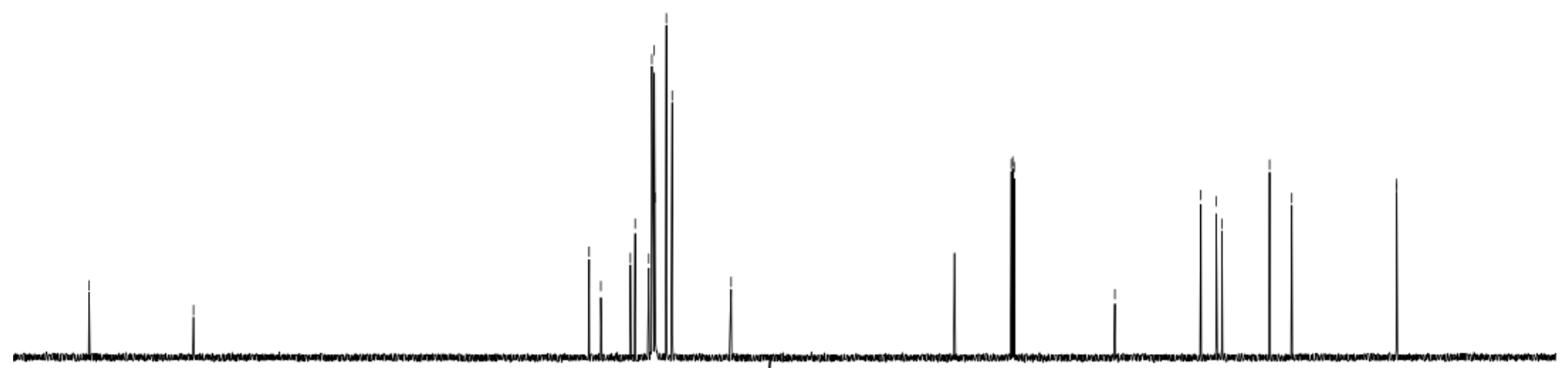

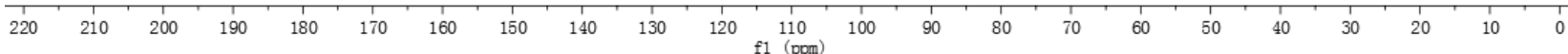




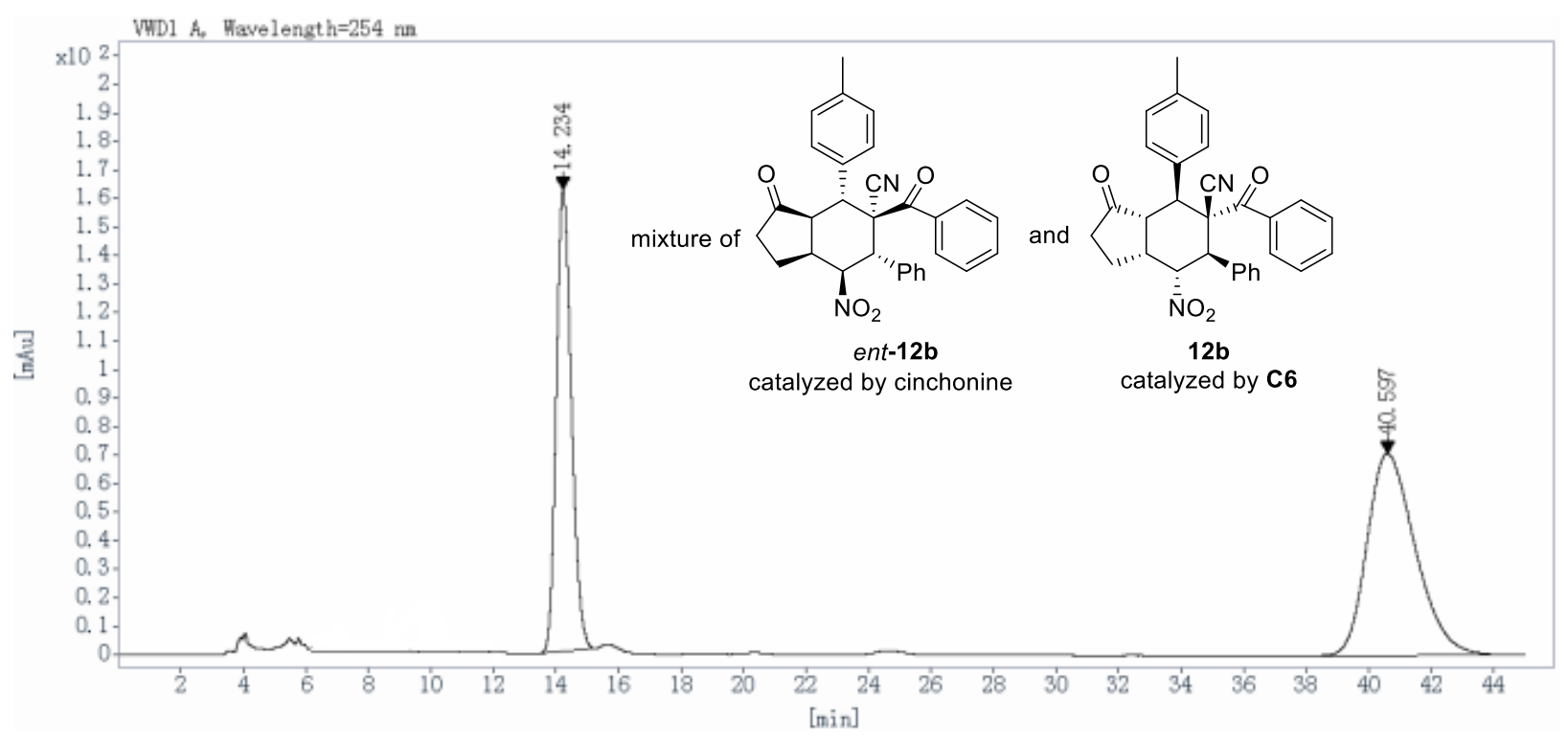

\begin{tabular}{cccccc}
$\begin{array}{c}\text { Ret Time } \\
{[\mathrm{min}]}\end{array}$ & $\begin{array}{c}\text { Peak } \\
\text { Type }\end{array}$ & $\begin{array}{c}\text { Width } \\
{[\mathrm{min}]}\end{array}$ & $\begin{array}{c}\text { Height } \\
{[\mathrm{mAU}]}\end{array}$ & $\begin{array}{c}\text { Area } \\
{\left[\mathrm{mAU}^{*} \mathbf{s}\right]}\end{array}$ & $\begin{array}{c}\text { Area } \\
{[\%]}\end{array}$ \\
\hline 14.234 & BB & 0.52 & 161.8310 & 5458.3267 & 41.7577 \\
40.597 & BBA & 1.64 & 70.7844 & 7613.1099 & 58.2423 \\
& & & Totals: & 13071.4365 & 100.0000
\end{tabular}

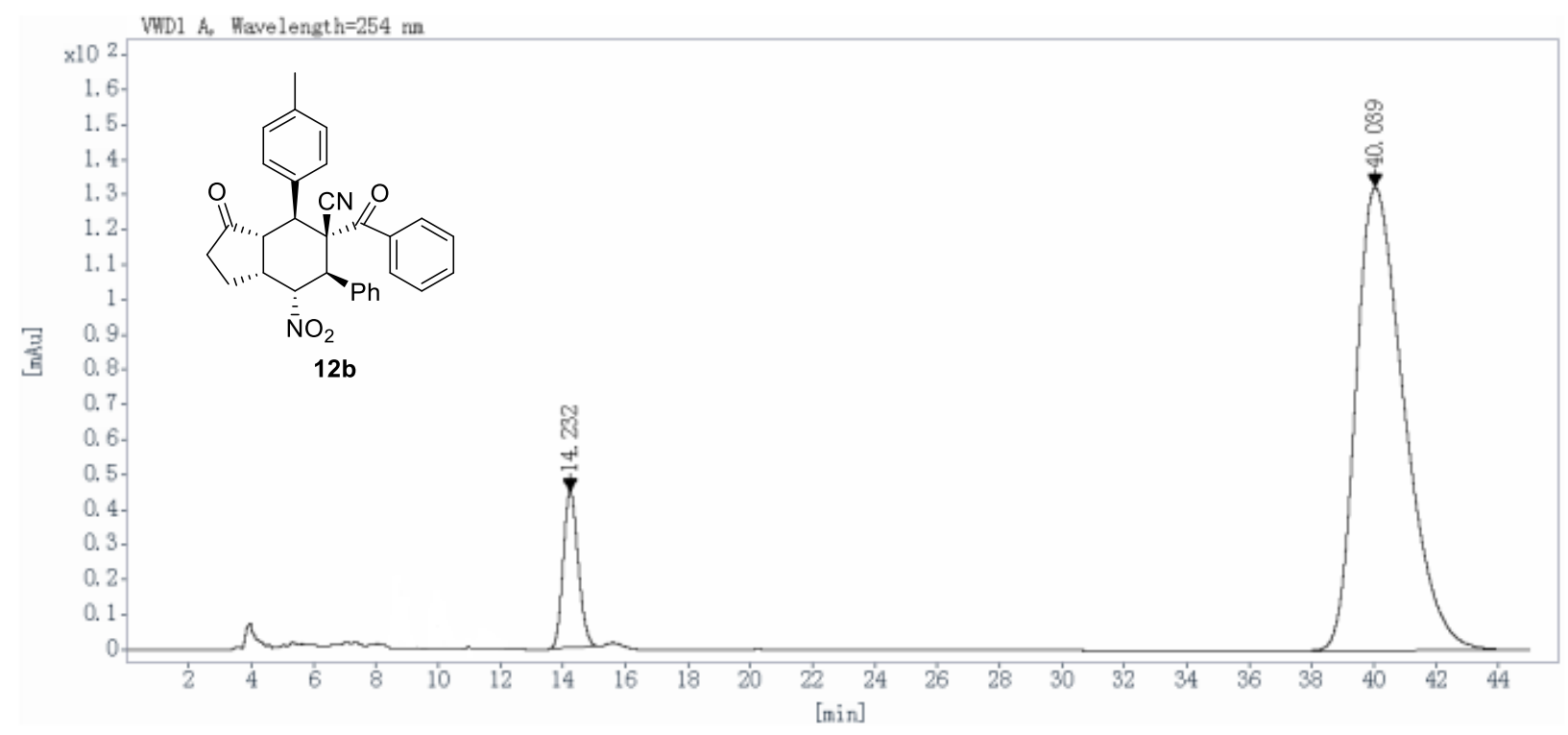

\begin{tabular}{cccccc}
$\begin{array}{c}\text { Ret Time } \\
\text { [min] }\end{array}$ & $\begin{array}{c}\text { Peak } \\
\text { Type }\end{array}$ & $\begin{array}{c}\text { Width } \\
{[\mathrm{min}]}\end{array}$ & $\begin{array}{c}\text { Height } \\
{[\mathrm{mAU}]}\end{array}$ & $\begin{array}{c}\text { Area } \\
{\left[\mathrm{mAU} \mathbf{U}^{*} \mathbf{s}\right]}\end{array}$ & $\begin{array}{c}\text { Area } \\
{[\%]}\end{array}$ \\
\hline 14.232 & BBA & 0.52 & 44.5588 & 1479.1577 & 9.4187 \\
40.039 & BBA & 1.64 & $\begin{array}{c}132.3924 \\
\text { Totals: }\end{array}$ & 14225.2979 & 90.5813 \\
& & & 15704.4556 & 100.0000
\end{tabular}


준?

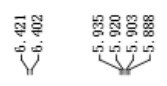

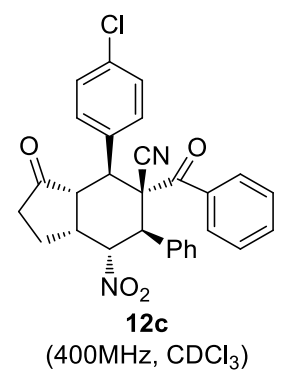

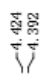

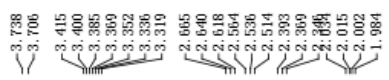
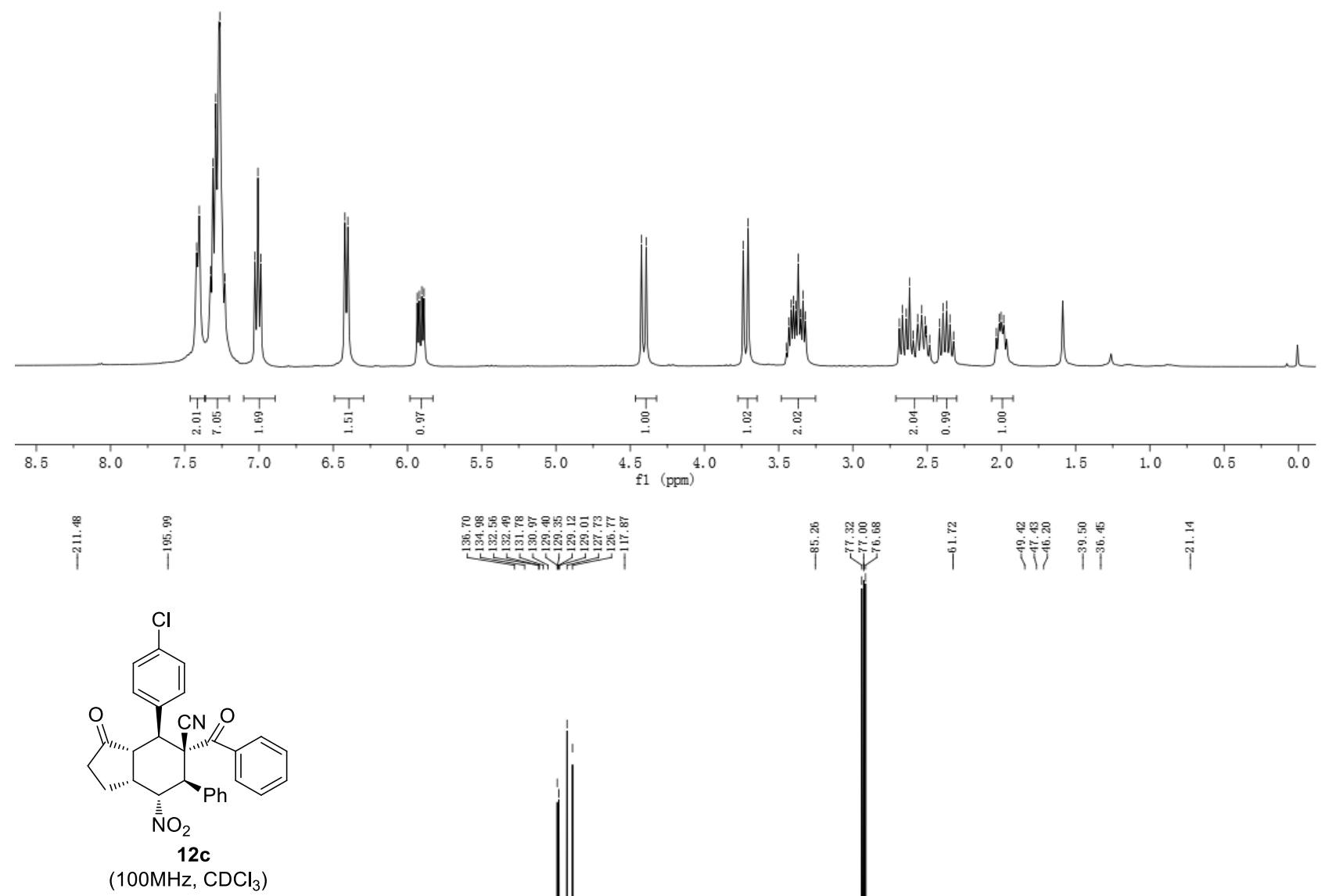

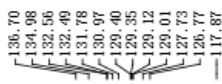

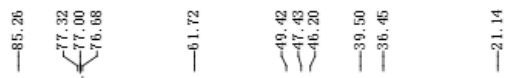
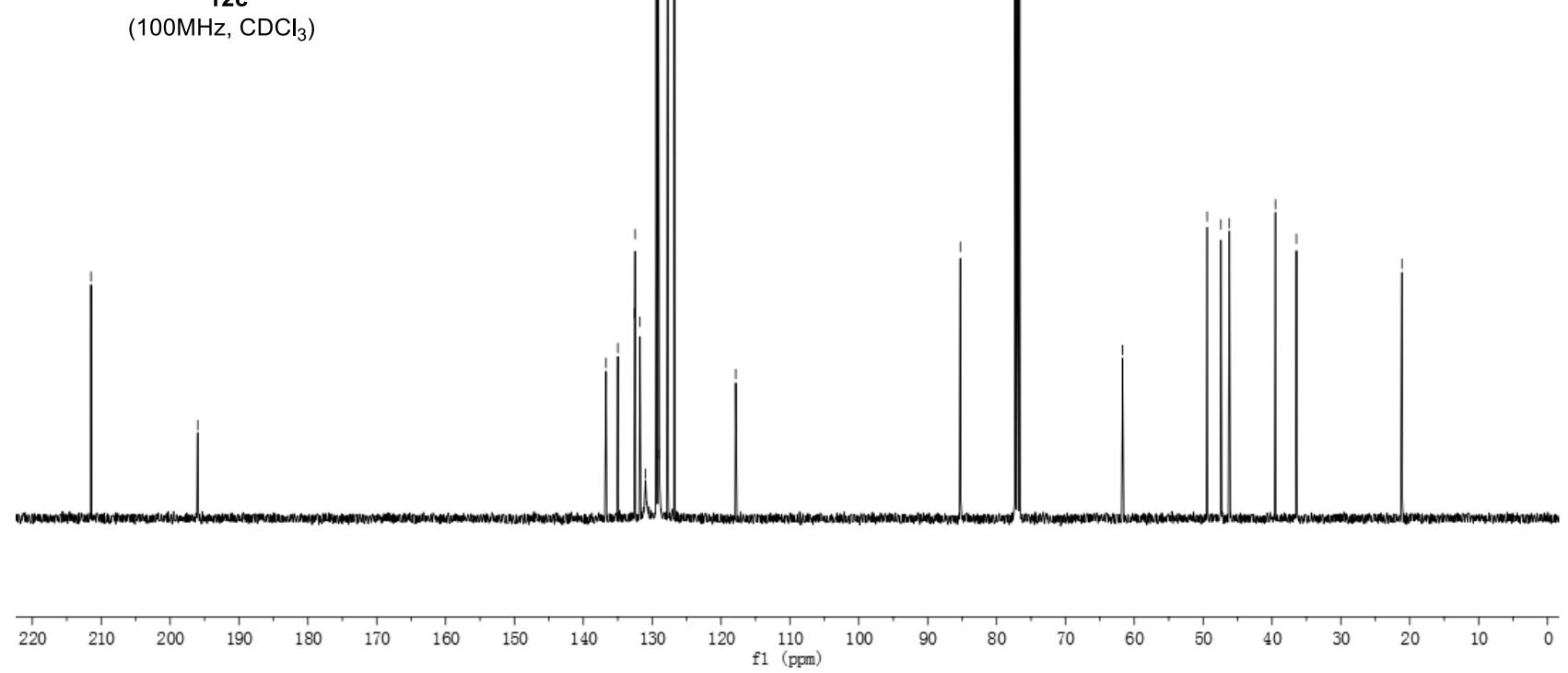

S109 


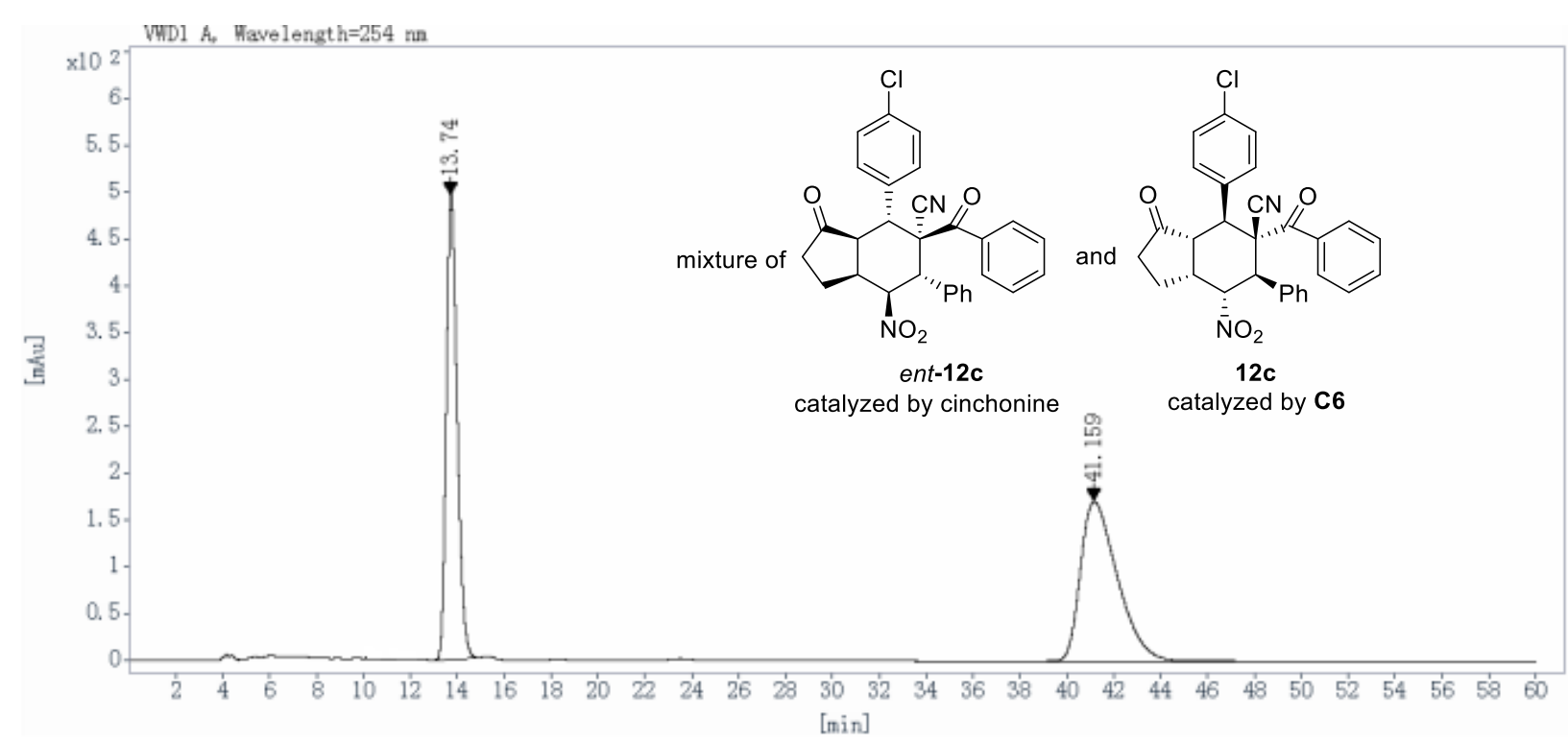

$\begin{array}{cccccc}\begin{array}{c}\text { Ret Time } \\ \text { [min] }\end{array} & \begin{array}{c}\text { Peak } \\ \text { Type }\end{array} & \begin{array}{c}\text { Width } \\ {[\mathrm{min}]}\end{array} & \begin{array}{c}\text { Height } \\ {[\mathrm{mAU}]}\end{array} & \begin{array}{c}\text { Area } \\ {[\mathrm{mAU} \text { s] }}\end{array} & \begin{array}{c}\text { Area } \\ {[\%]}\end{array} \\ 13.740 & \text { BB } & 0.50 & 495.3008 & 15924.6426 & 46.0731 \\ 41.159 & \text { BB } & 1.67 & 170.3046 & 18639.2207 & 53.9269 \\ & & & \text { Totals: } & 34563.8633 & 100.0000\end{array}$

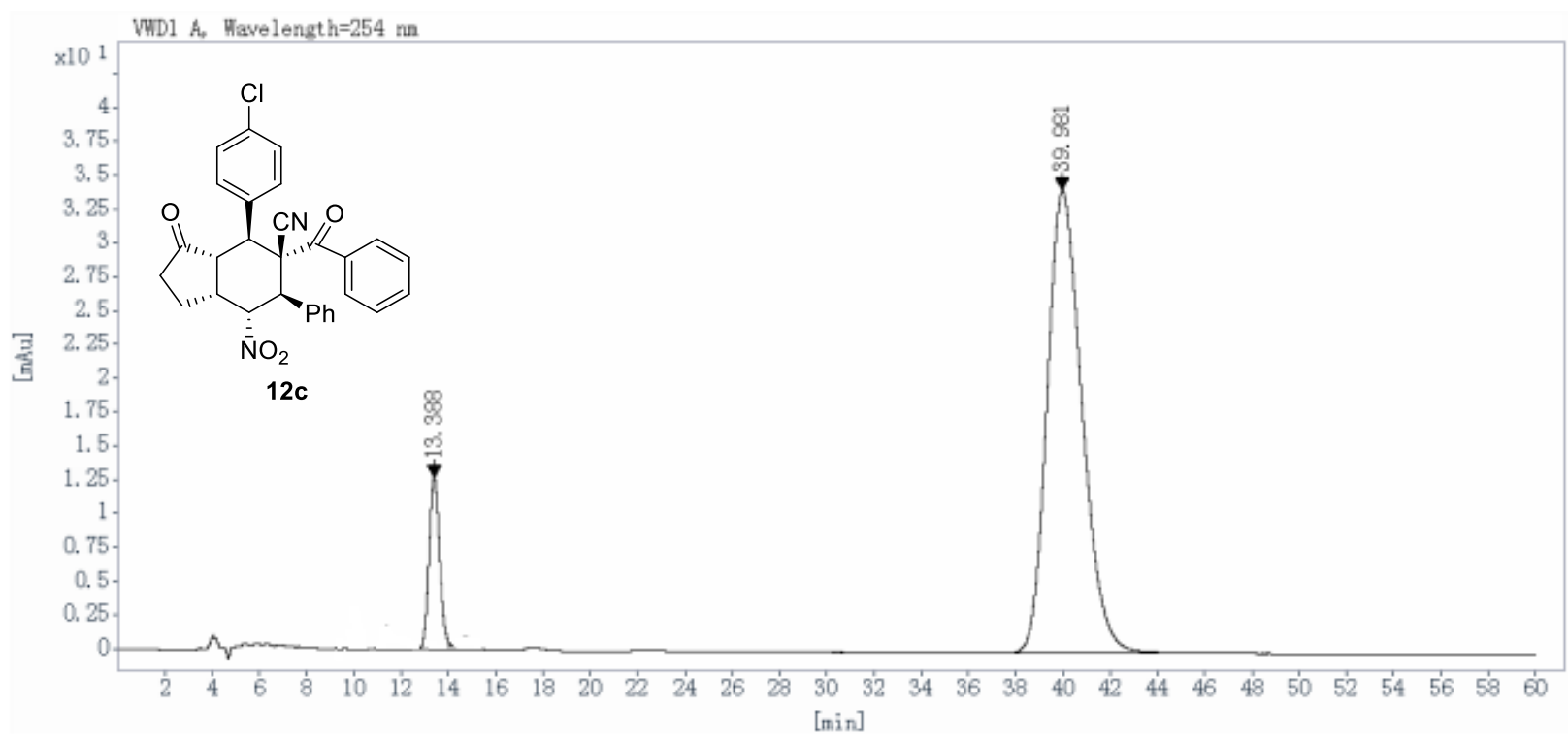

\begin{tabular}{cccccc}
$\begin{array}{c}\text { Ret Time } \\
\text { [min] }\end{array}$ & $\begin{array}{c}\text { Peak } \\
\text { Type }\end{array}$ & $\begin{array}{c}\text { Width } \\
{[\mathrm{min}]}\end{array}$ & $\begin{array}{c}\text { Height } \\
{[\mathrm{mAU}]}\end{array}$ & $\begin{array}{c}\text { Area } \\
{\left[\mathrm{mAU}^{*} \mathbf{s}\right]}\end{array}$ & $\begin{array}{c}\text { Area } \\
{[\%]}\end{array}$ \\
\hline 13.388 & BB & 0.48 & 12.5945 & 386.5583 & 9.9662 \\
39.981 & BB & 1.57 & 34.1107 & 3492.1396 & 90.0338 \\
& & & Totals: & 3878.6979 & 100.0000
\end{tabular}




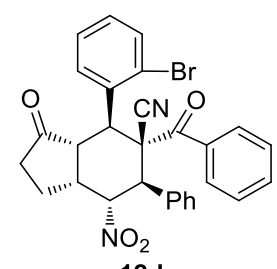

$\left(400 \mathrm{MHz}, \mathrm{CDCl}_{3}\right)$

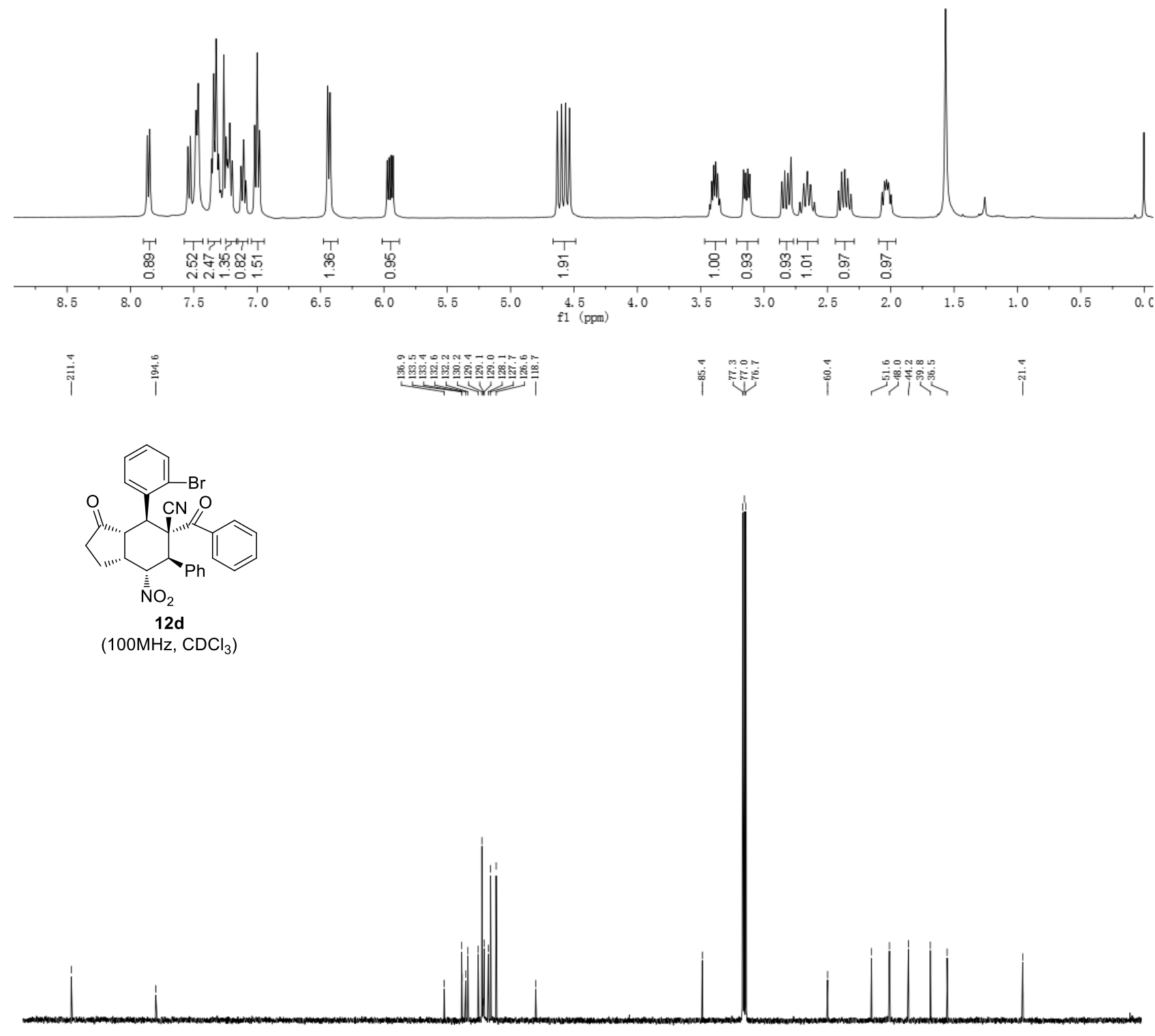

$\stackrel{1}{20}$
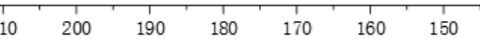

140 f1 $(10 \mathrm{pm})$ 


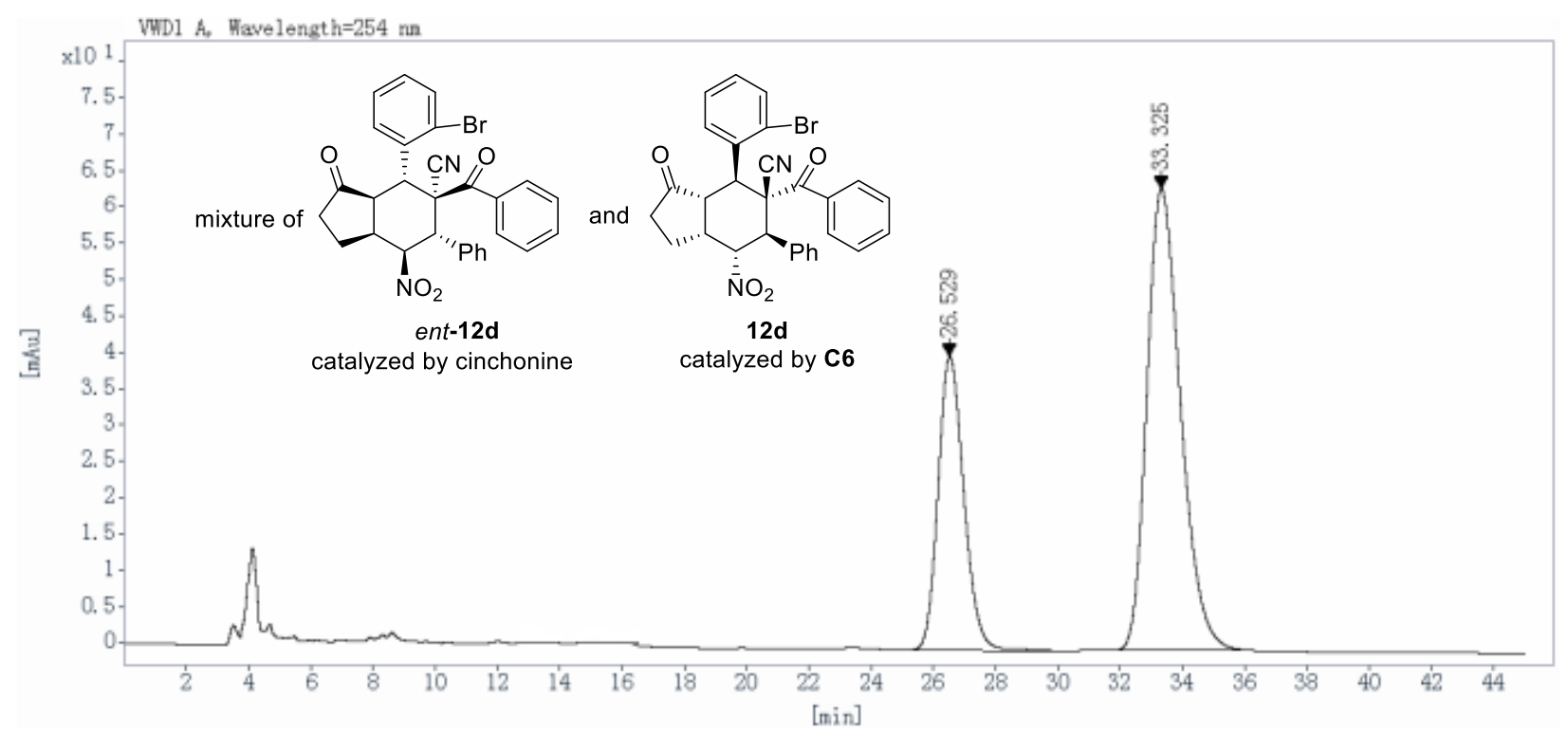

$\begin{array}{cccccc}\begin{array}{c}\text { Ret Time } \\ \text { [min] }\end{array} & \begin{array}{c}\text { Peak } \\ \text { Type }\end{array} & \begin{array}{c}\text { Width } \\ {[\mathrm{min}]}\end{array} & \begin{array}{c}\text { Height } \\ {[\mathrm{mAU}]}\end{array} & \begin{array}{c}\text { Area } \\ {\left[\mathrm{mAU} \mathbf{A}^{*} \text { ] }\right.}\end{array} & \begin{array}{c}\text { Area } \\ {[\%]}\end{array} \\ 26.529 & \text { BB } & 0.88 & 40.3576 & 2322.3757 & 32.8290 \\ 33.325 & \text { BBA } & 1.13 & 63.3756 & 4751.7910 & 67.1710 \\ & & & \text { Totals: } & 7074.1667 & 100.0000\end{array}$

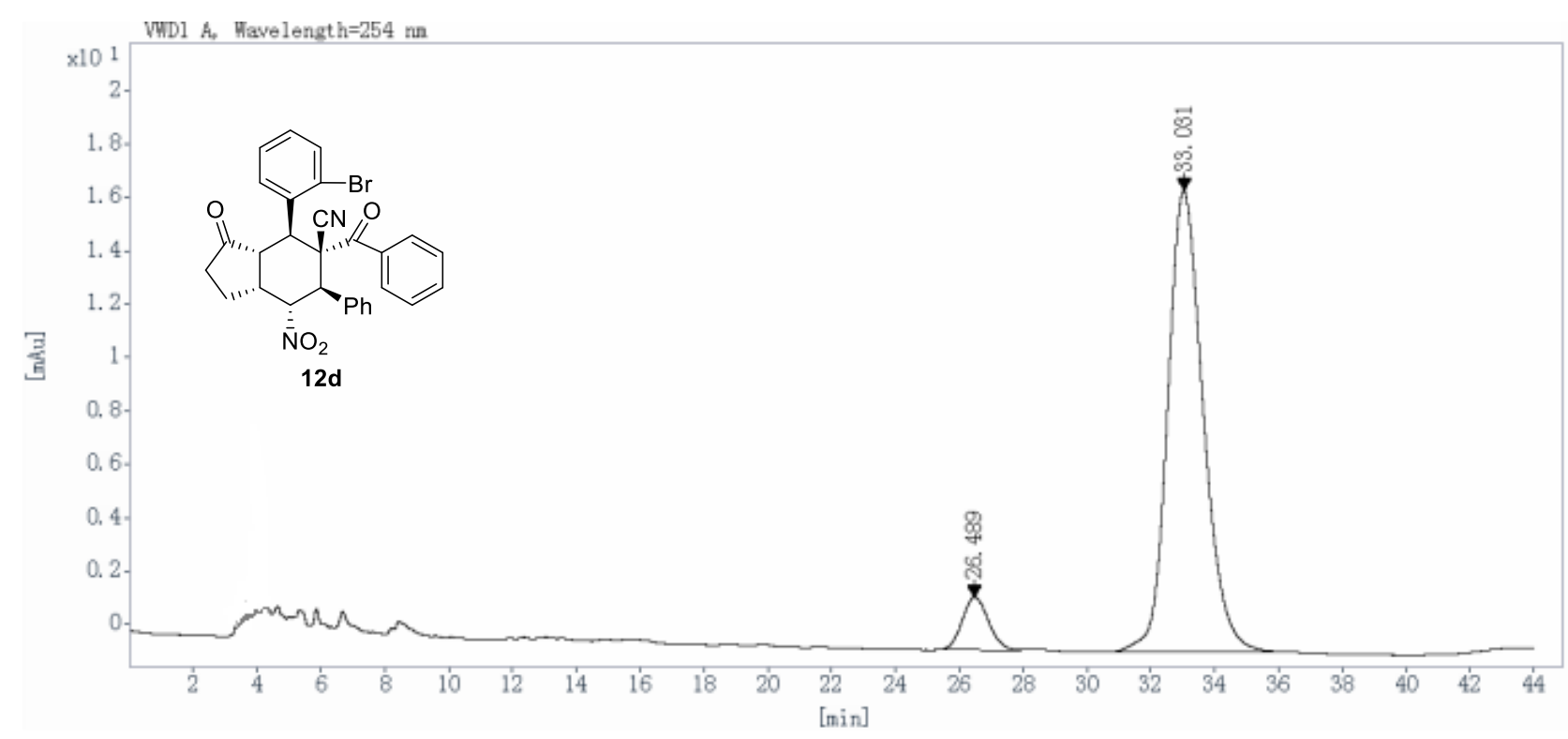

$\begin{array}{cccccc}\begin{array}{c}\text { Ret Time } \\ {[\mathrm{min}]}\end{array} & \begin{array}{c}\text { Peak } \\ \text { Type }\end{array} & \begin{array}{c}\text { Width } \\ {[\mathrm{min}]}\end{array} & \begin{array}{c}\text { Height } \\ {[\mathrm{mAU}]}\end{array} & \begin{array}{c}\text { Area } \\ {\left[\mathrm{mAU}^{*} \mathrm{~s}\right]}\end{array} & \begin{array}{c}\text { Area } \\ {[\%]}\end{array} \\ 26.489 & \text { BB } & 0.78 & 1.9720 & 112.1931 & 7.7414 \\ 33.031 & \text { BB } & 1.17 & 17.2605 & 1337.0769 & 92.2586 \\ & & & \text { Totals: } & 1449.2701 & 100.0000\end{array}$




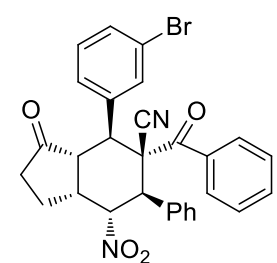

$\left(400 \mathrm{MHz}, \mathrm{CDCl}_{3}\right)$

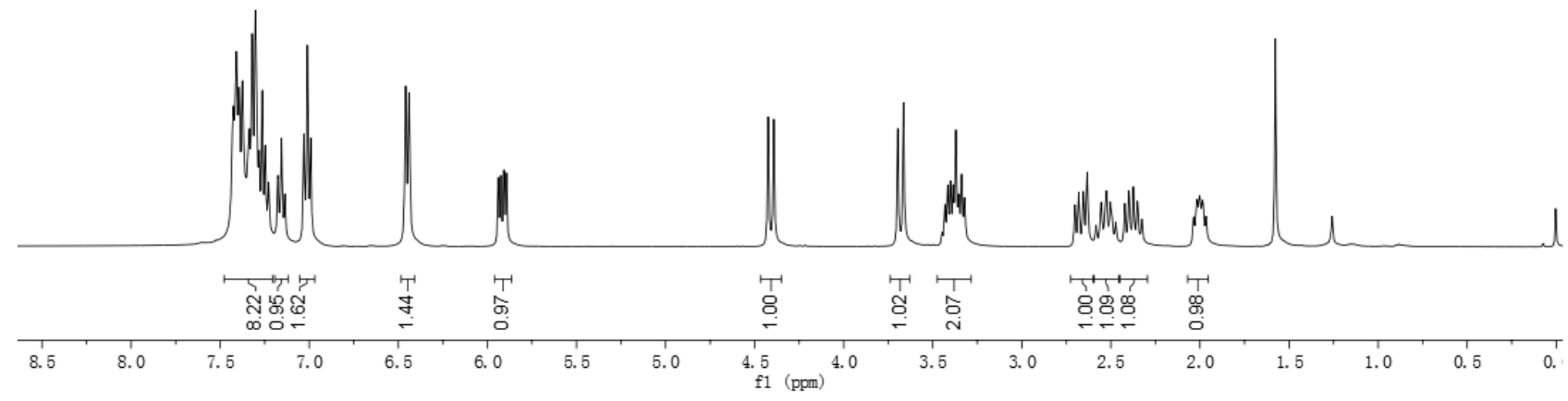

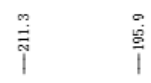

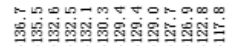

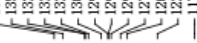

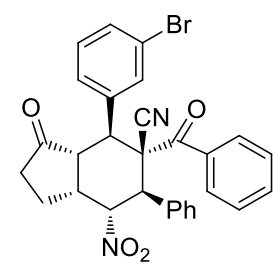

$12 \mathrm{e}$

$\left(100 \mathrm{MHz}, \mathrm{CDCl}_{3}\right)$

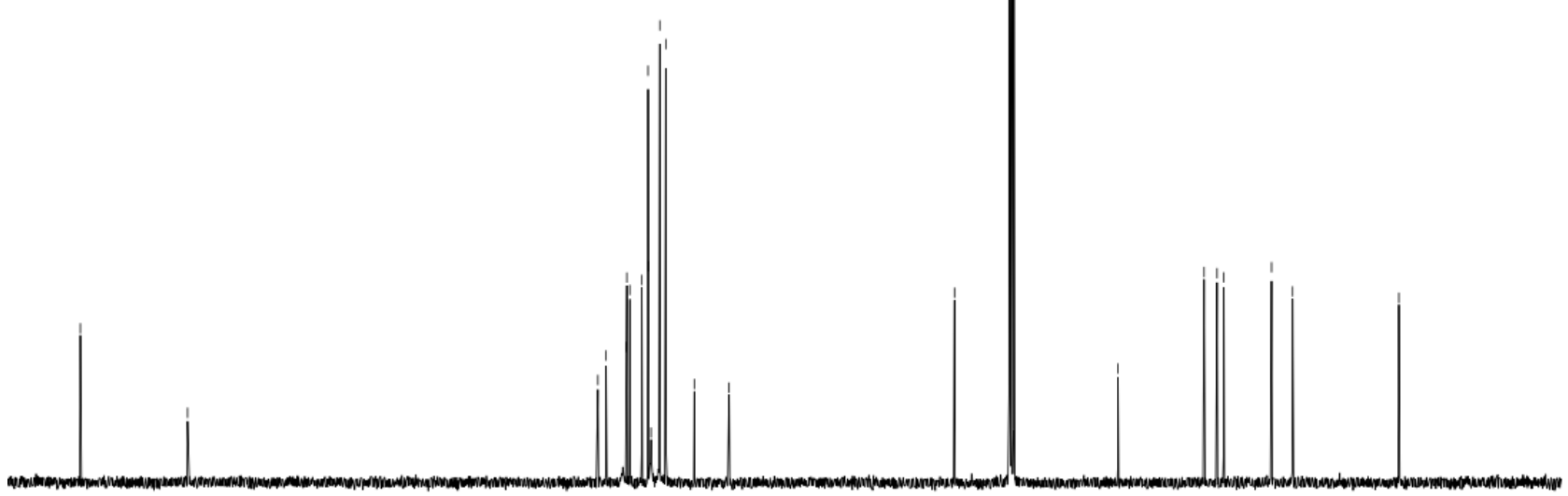

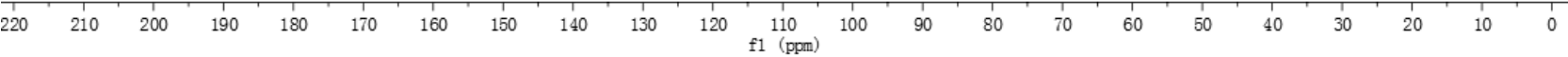




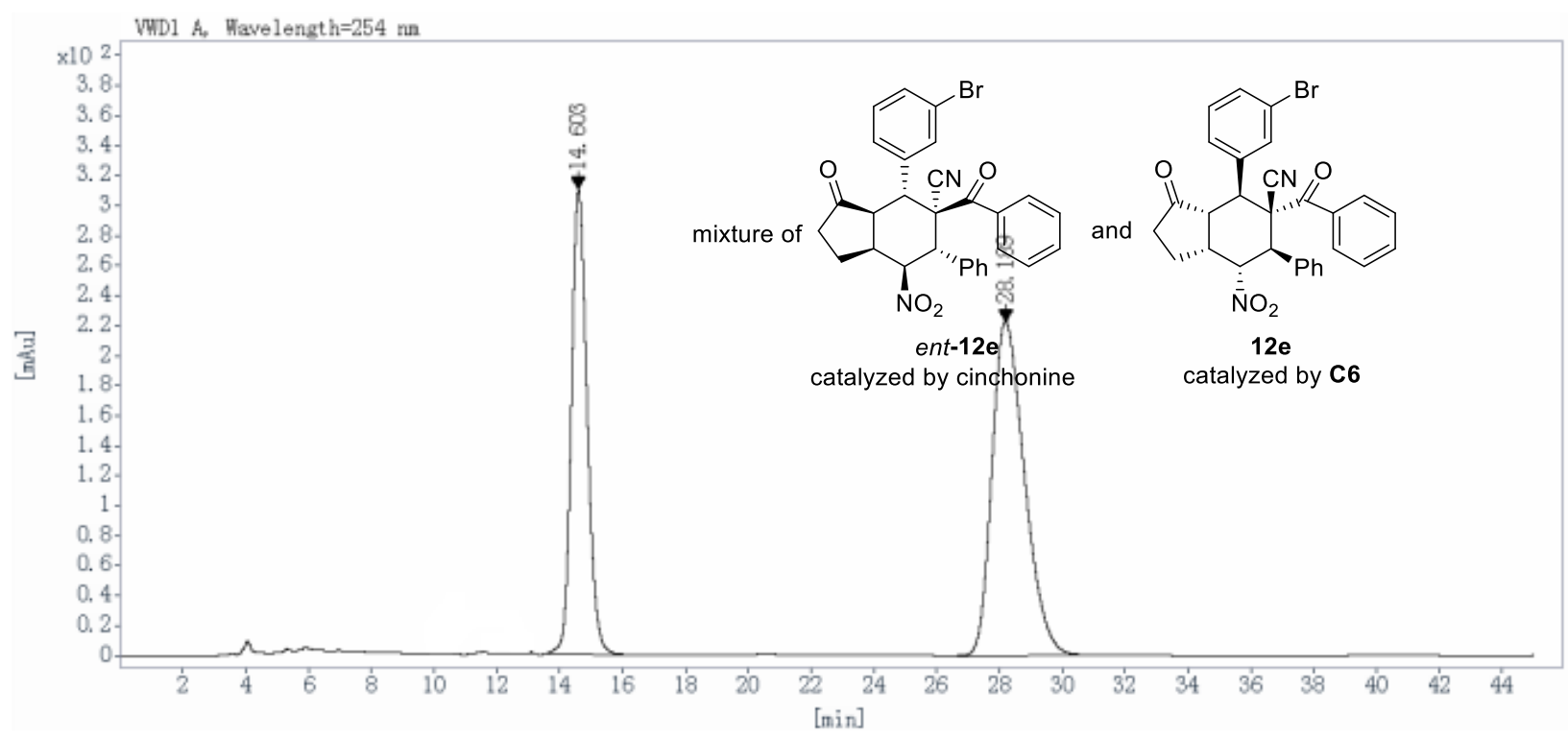

\begin{tabular}{cccccc}
$\begin{array}{c}\text { Ret Time } \\
{[\mathrm{min}]}\end{array}$ & $\begin{array}{c}\text { Peak } \\
\text { Type }\end{array}$ & $\begin{array}{c}\text { Width } \\
{[\mathrm{min}]}\end{array}$ & $\begin{array}{c}\text { Height } \\
{[\mathrm{mAU}]}\end{array}$ & $\begin{array}{c}\text { Area } \\
{\left[\mathrm{mAU}{ }^{*} \text { s] }\right.}\end{array}$ & $\begin{array}{c}\text { Area } \\
{[\%]}\end{array}$ \\
\hline 14.603 & BB & 0.53 & 309.4417 & 10716.9961 & 40.0750 \\
28.189 & BB & 1.11 & 222.6176 & 16025.3486 & 59.9250 \\
& & & Totals: & 26742.3447 & 100.0000
\end{tabular}

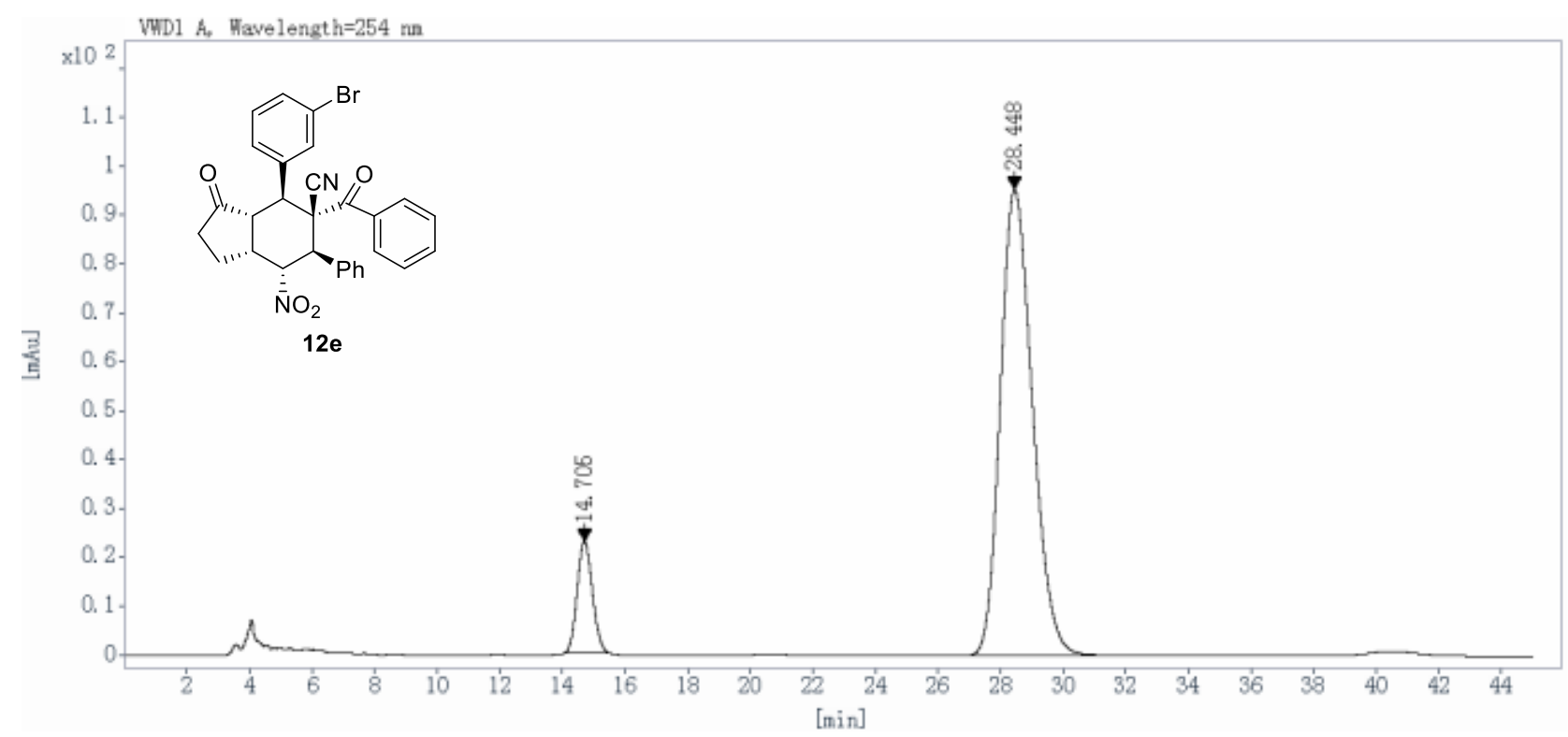

$\begin{array}{cccccc}\begin{array}{c}\text { Ret Time } \\ {[\mathrm{min}]}\end{array} & \begin{array}{c}\text { Peak } \\ \text { Type }\end{array} & \begin{array}{c}\text { Width } \\ {[\mathrm{min}]}\end{array} & \begin{array}{c}\text { Height } \\ {[\mathrm{mAU}]}\end{array} & \begin{array}{c}\text { Area } \\ {\left[\mathrm{mAU}^{*} \text { s }\right]}\end{array} & \begin{array}{c}\text { Area } \\ {[\%]}\end{array} \\ 14.705 & \text { BBA } & 0.53 & 22.8103 & 768.6624 & 10.1246 \\ 28.448 & \text { BB } & 1.11 & 95.3737 & 6823.3579 & 89.8754 \\ & & & \text { Totals: } & 7592.0203 & 100.0000\end{array}$




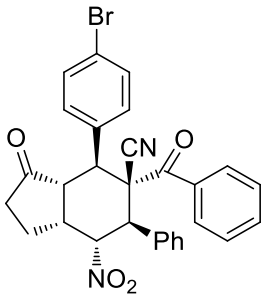

$12 f$

$\left(400 \mathrm{MHz}, \mathrm{CDCl}_{3}\right)$

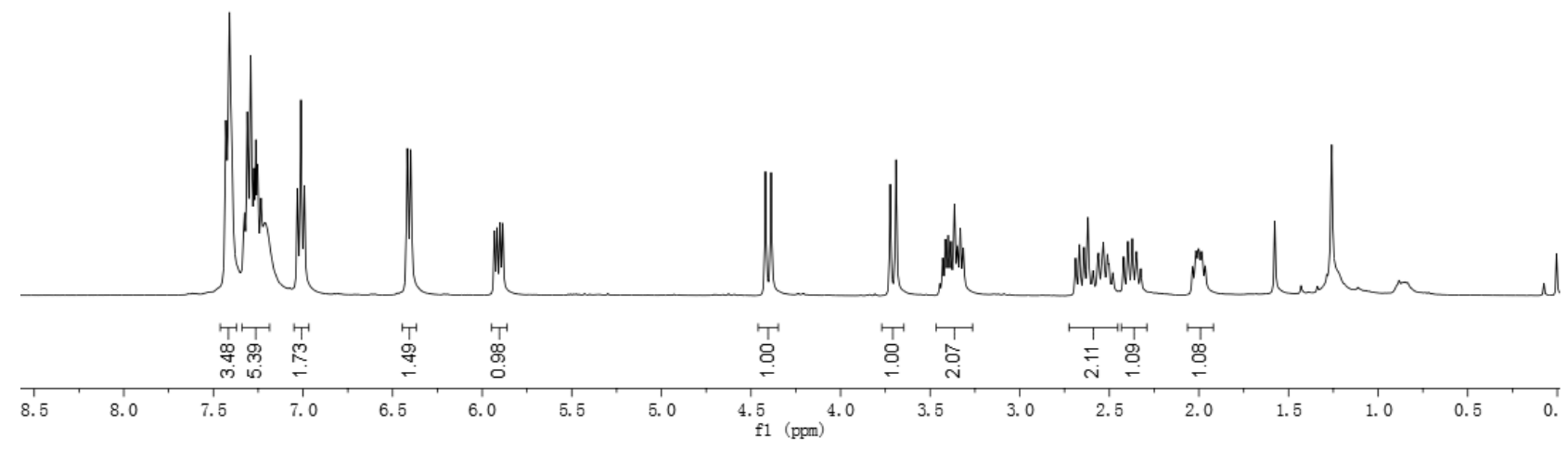

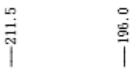

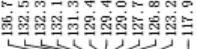

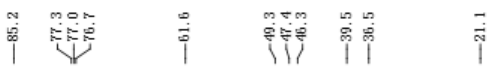

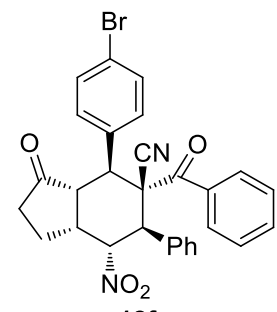

$12 f$

$\left(100 \mathrm{MHz}, \mathrm{CDCl}_{3}\right.$ )

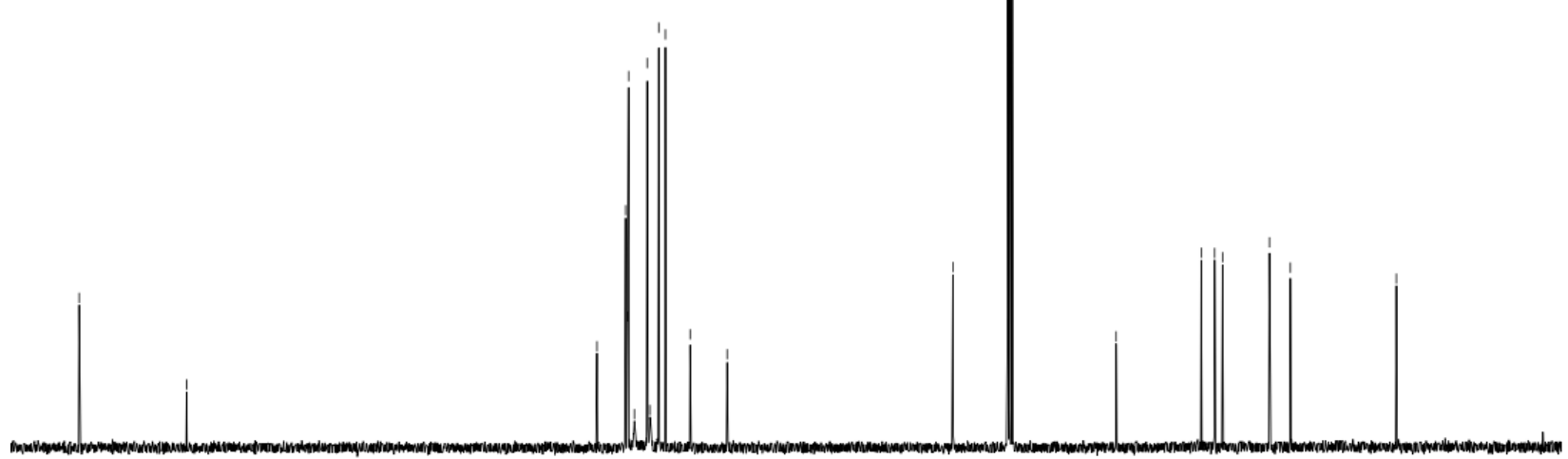

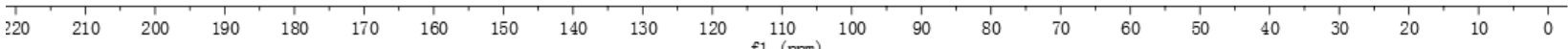




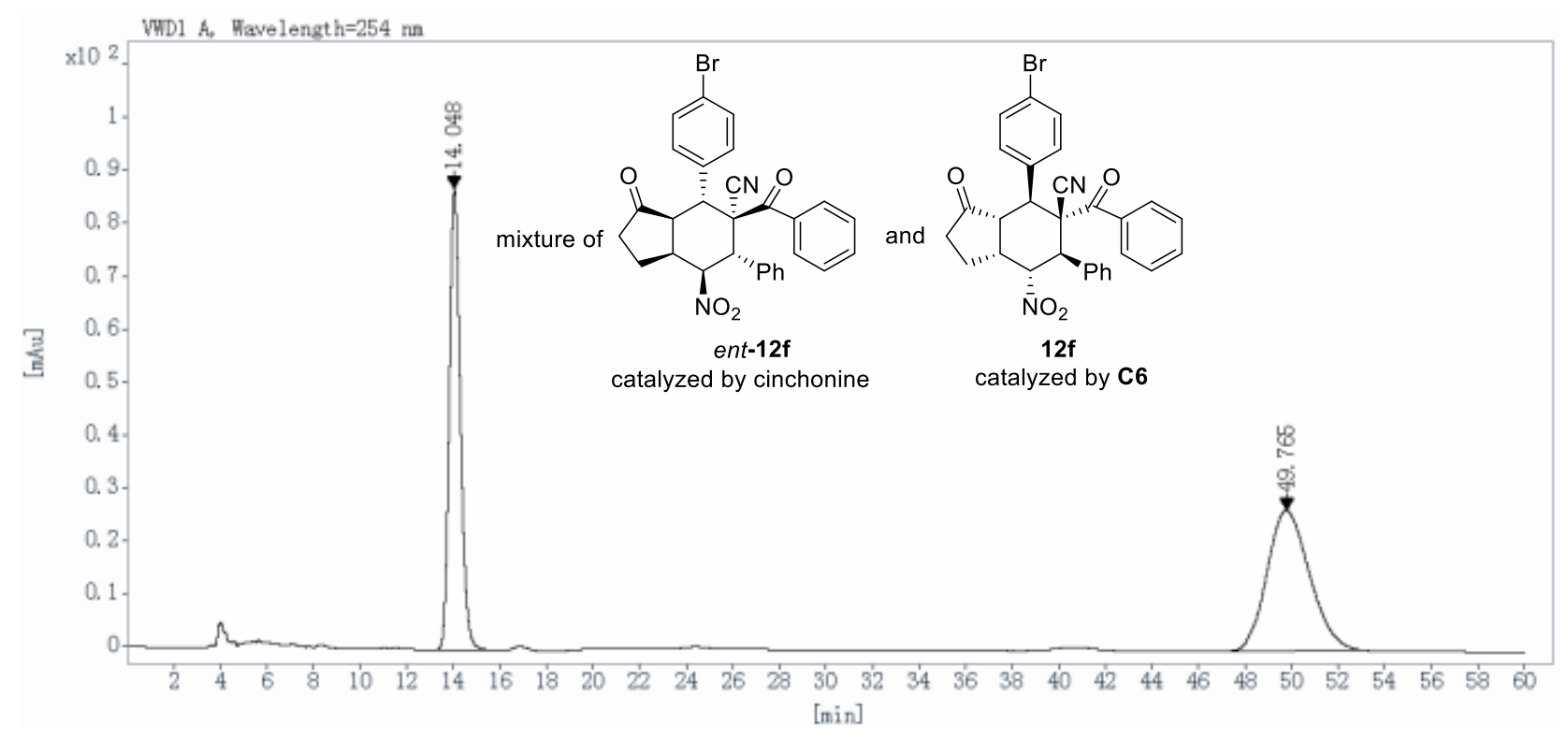

\begin{tabular}{|c|c|c|c|c|c|}
\hline $\begin{array}{l}\text { Ret Time } \\
\text { [min] }\end{array}$ & $\begin{array}{l}\text { Peak } \\
\text { Type }\end{array}$ & $\begin{array}{l}\text { Width } \\
\text { [min] }\end{array}$ & $\begin{array}{l}\text { Height } \\
\text { [mAU] }\end{array}$ & $\begin{array}{c}\text { Area } \\
{\left[\mathbf{m A U}^{*} \mathbf{s}\right]}\end{array}$ & $\begin{array}{l}\text { Area } \\
{[\%]}\end{array}$ \\
\hline 14.048 & BB & 0.51 & 87.0748 & 2878.2803 & 46.1540 \\
\hline 49.765 & BB & 1.82 & 26.4309 & 3357.9717 & 53.8460 \\
\hline & & & Totals: & 6236.2520 & 100.0000 \\
\hline
\end{tabular}

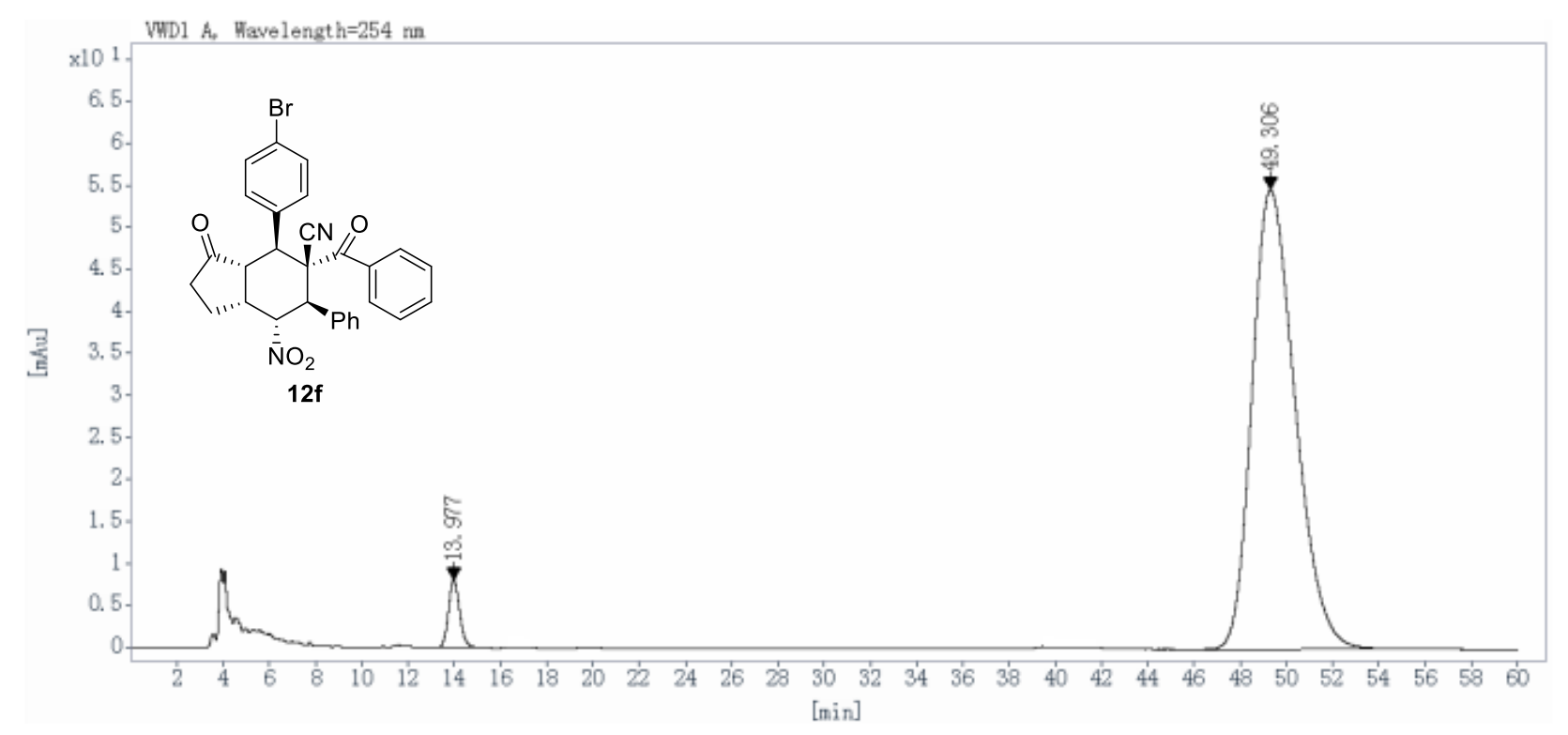

\begin{tabular}{cccccc}
$\begin{array}{c}\text { Ret Time } \\
{[\mathrm{min}]}\end{array}$ & $\begin{array}{c}\text { Peak } \\
\text { Type }\end{array}$ & $\begin{array}{c}\text { Width } \\
{[\mathrm{min}]}\end{array}$ & $\begin{array}{c}\text { Height } \\
{[\mathrm{mAU}]}\end{array}$ & $\begin{array}{c}\text { Area } \\
{\left[\mathrm{mAU}^{*} \mathrm{~s}\right]}\end{array}$ & $\begin{array}{c}\text { Area } \\
{[\%]}\end{array}$ \\
\hline 13.977 & BBA & 0.52 & 8.0256 & 270.7563 & 3.6868 \\
49.306 & BB & 1.90 & 54.6272 & 7073.1079 & 96.3132 \\
& & & Totals: & 7343.8642 & 100.0000
\end{tabular}



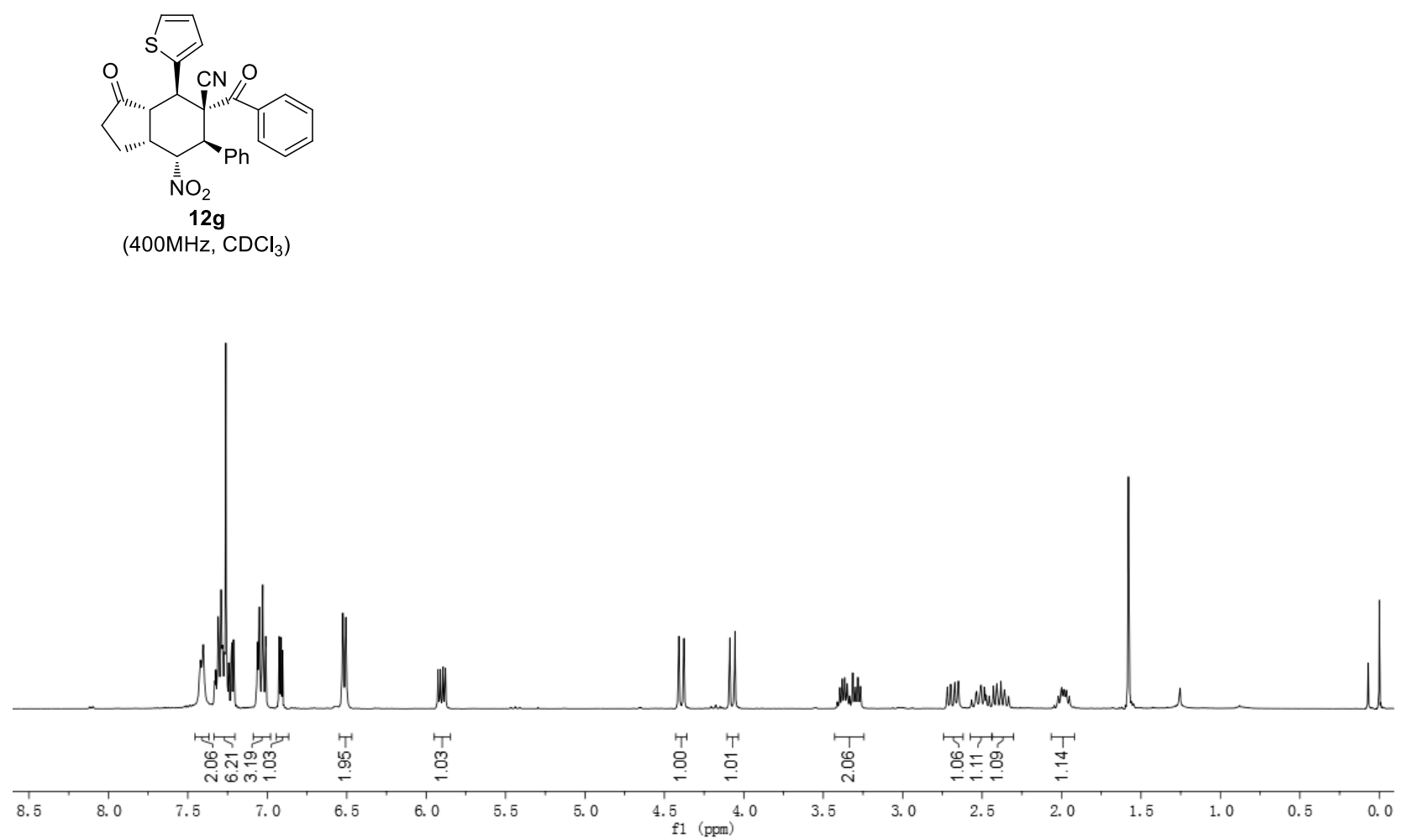

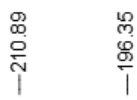

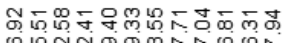

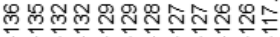

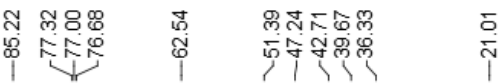

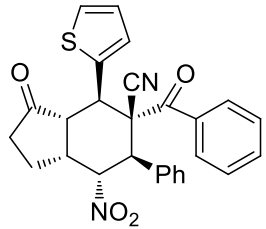

$12 \mathrm{~g}$

$\left(100 \mathrm{MHz}, \mathrm{CDCl}_{3}\right)$

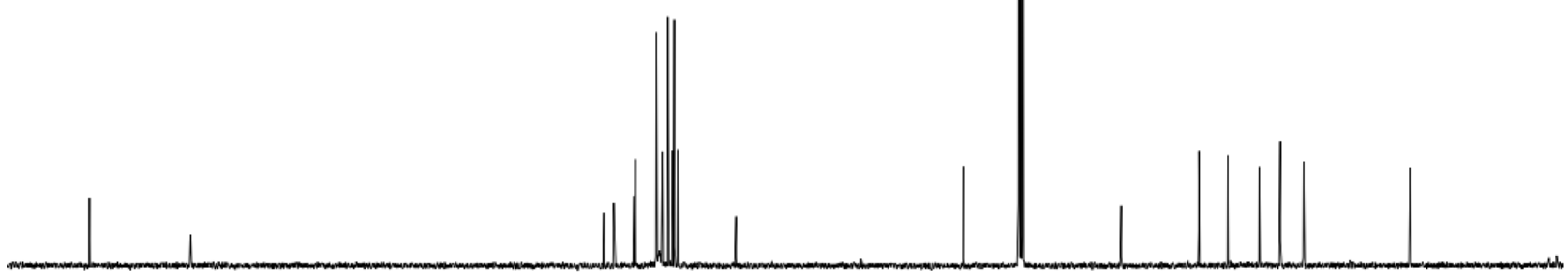

$\begin{array}{lllllllllllllllllllllllll}220 & 210 & 200 & 190 & 180 & 170 & 160 & 150 & 140 & 130 & 120 & 110 & 100 & 90 & 80 & 70 & 60 & 50 & 40 & 30 & 20 & 10 & 0\end{array}$ 


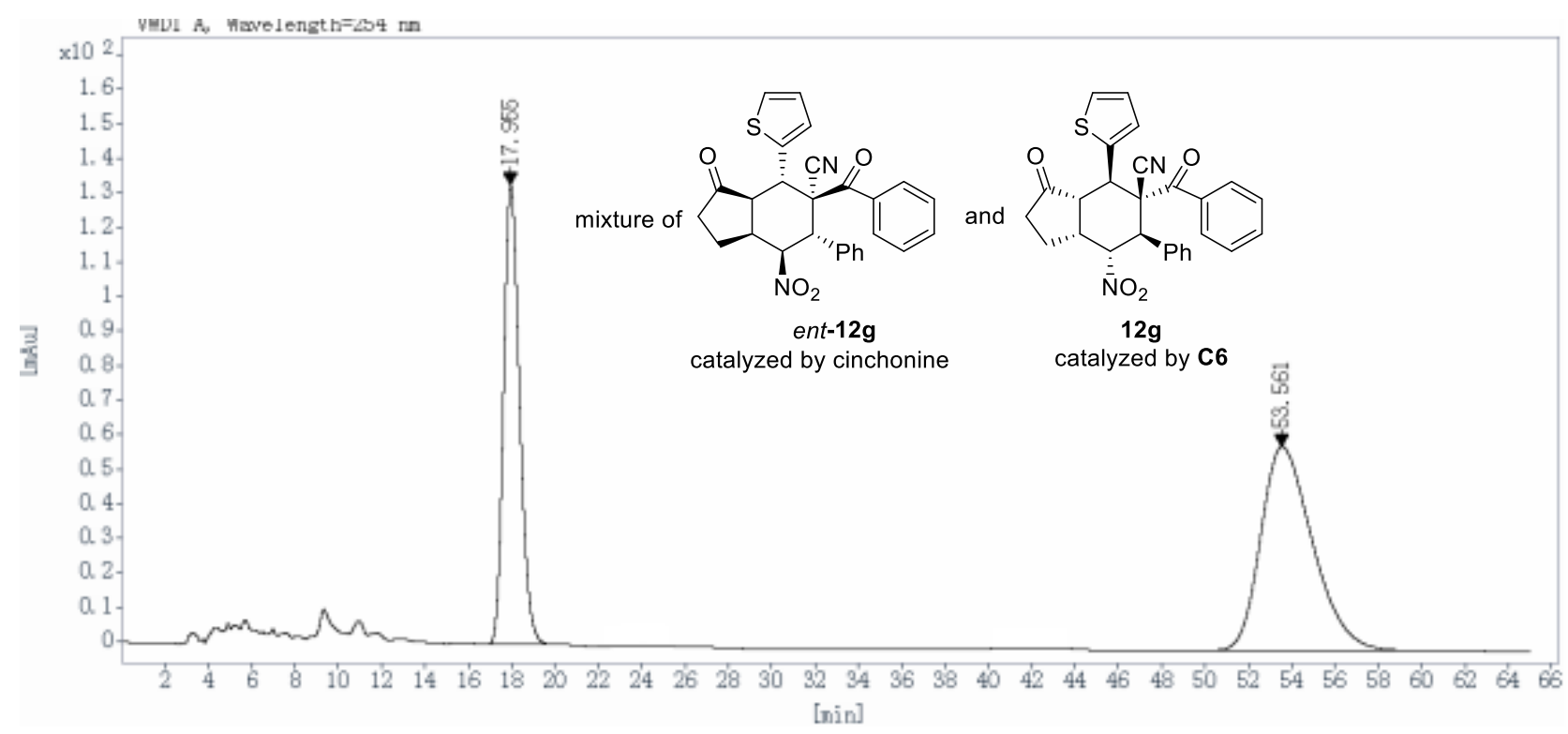

$\begin{array}{cccccc}\begin{array}{c}\text { Ret Time } \\ \text { [min] }\end{array} & \begin{array}{c}\text { Peak } \\ \text { Type }\end{array} & \begin{array}{c}\text { Width } \\ {[\mathrm{min}]}\end{array} & \begin{array}{c}\text { Height } \\ {[\mathrm{mAU}]}\end{array} & \begin{array}{c}\text { Area } \\ {\left[\mathrm{mAU} \mathbf{U}^{*} \mathrm{~s}\right]}\end{array} & \begin{array}{c}\text { Area } \\ {[\%]}\end{array} \\ 17.955 & \text { BB } & 0.79 & 133.0663 & 6816.8457 & 40.9767 \\ 53.561 & \text { BB } & 2.45 & 59.0161 & 9819.0713 & 59.0233 \\ & & & \text { Totals: } & 16635.9170 & 100.0000\end{array}$

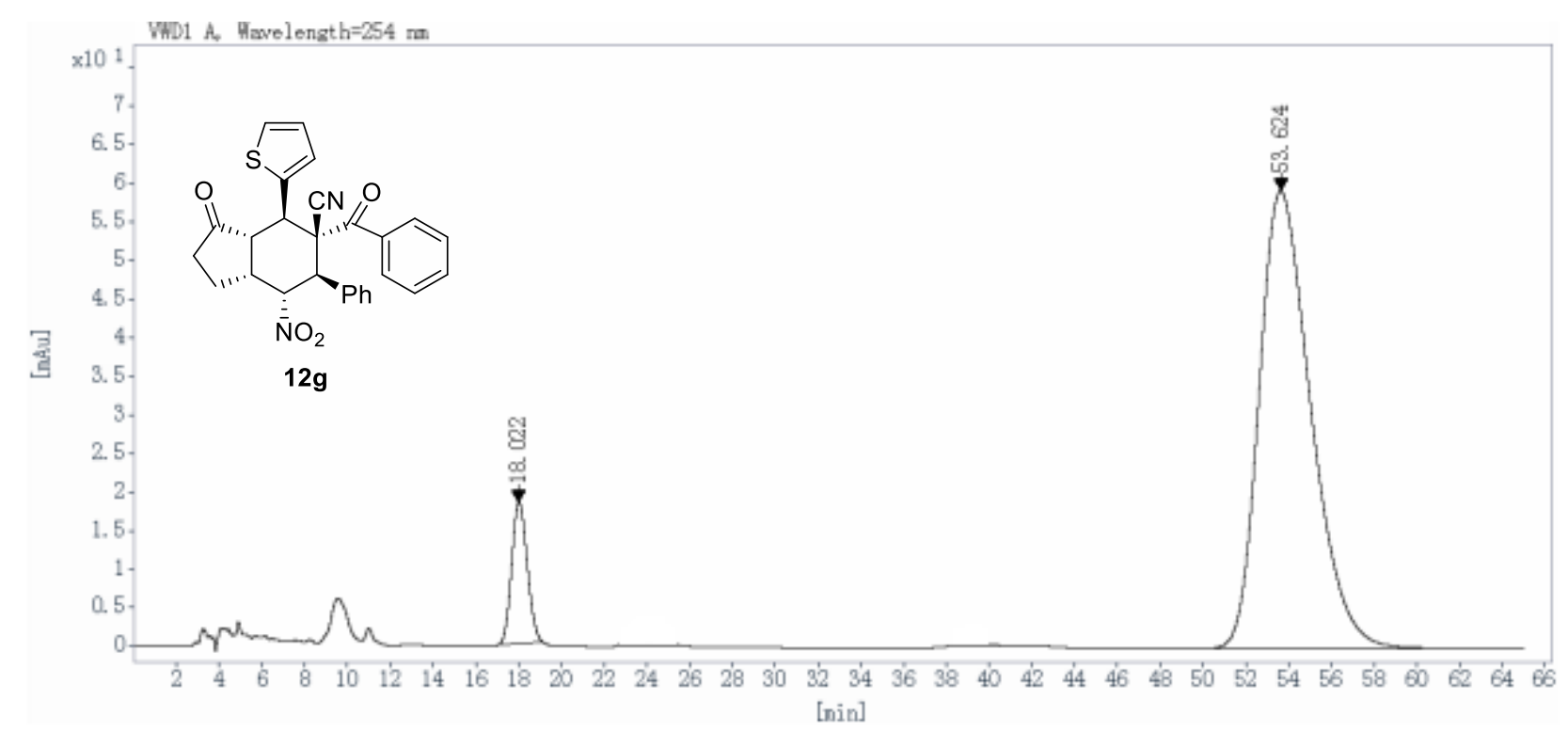

$\begin{array}{cccccc}\begin{array}{c}\text { Ret Time } \\ \text { [min] }\end{array} & \begin{array}{c}\text { Peak } \\ \text { Type }\end{array} & \begin{array}{c}\text { Width } \\ {[\mathrm{min}]}\end{array} & \begin{array}{c}\text { Height } \\ {[\mathrm{mAU}]}\end{array} & \begin{array}{c}\text { Area } \\ {\left[\mathrm{mAU} \mathbf{A}^{*}\right]}\end{array} & \begin{array}{c}\text { Area } \\ {[\%]}\end{array} \\ 18.022 & \text { BBA } & 0.78 & 18.3559 & 926.4836 & 8.5706 \\ 53.624 & \text { BB } & 2.44 & 59.1773 & 9883.5273 & 91.4294 \\ & & & \text { Totals: } & 10810.0110 & 100.0000\end{array}$


<smiles>Cc1ccc(C(=O)N[C@@]2(C(=O)c3ccc(Br)cc3)[C@H](c3ccc(Br)cc3)[C@H]3C(=O)CC[C@H]3[C@H]([N+](=O)[O-])[C@@H]2c2ccccc2)cc1</smiles>

$12 \mathrm{~h}$

(400MHz, $\mathrm{CDCl}_{3}$ )

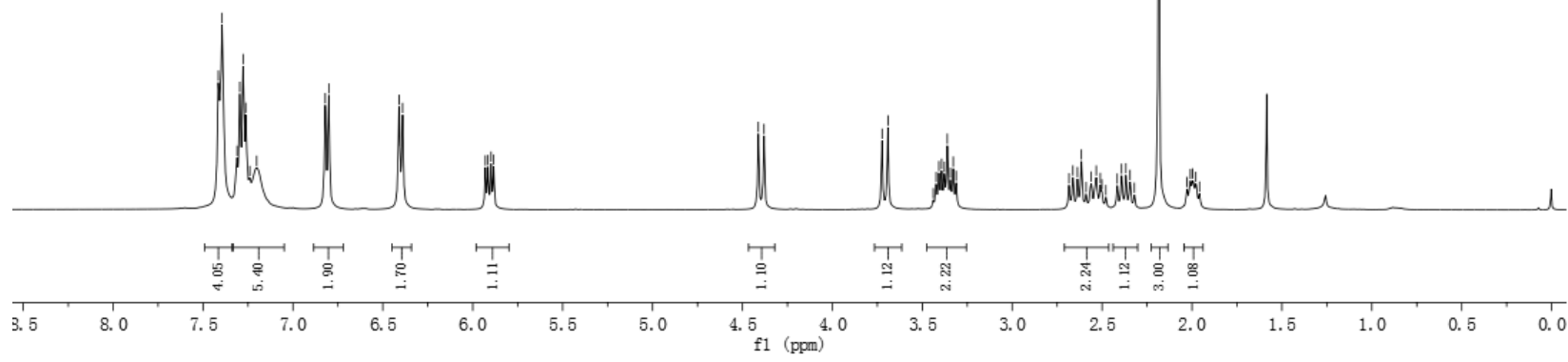

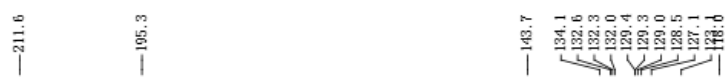<smiles>Cc1ccc(C(=O)N(C)C(C)(C)[C@]2(c3ccc(Br)cc3)[C@H](c3ccc(Br)cc3)[C@H]3CCC(=O)[C@H]3[C@@H]2[N+](=O)[O-])cc1</smiles>

$12 \mathrm{~h}$

$\left(100 \mathrm{MHz} \mathrm{CDCl}_{3}\right)$

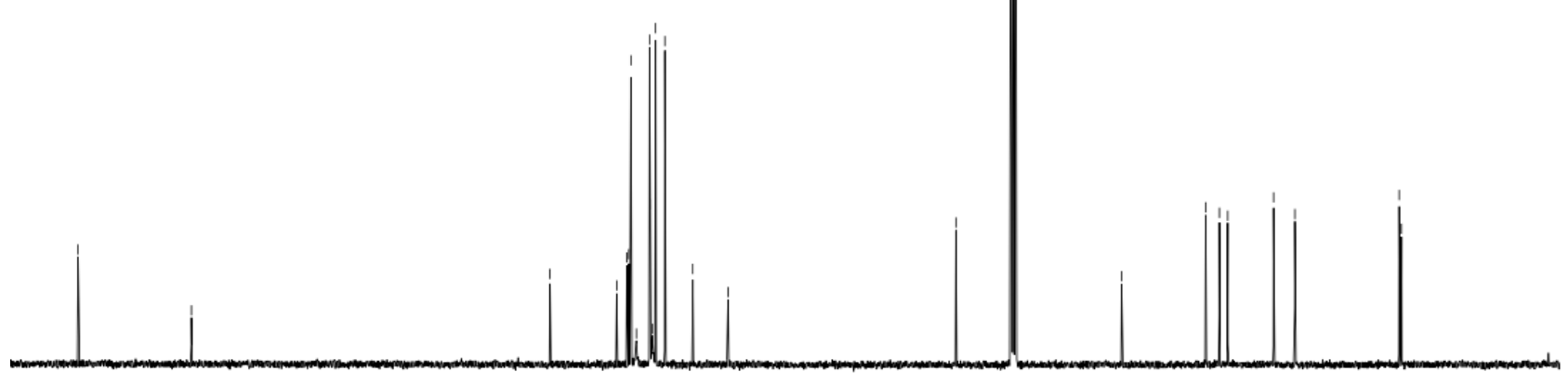

$\begin{array}{lllllllllllllllllllllllll}1 & 1 \\ 220 & 210 & 200 & 190 & 180 & 170 & 160 & 150 & 140 & 130 & 120 & 110 & 100 & 90 & 80 & 70 & 60 & 50 & 40 & 30 & 1 & 1 & 1 & 1 & 1 \\ 1\end{array}$ 


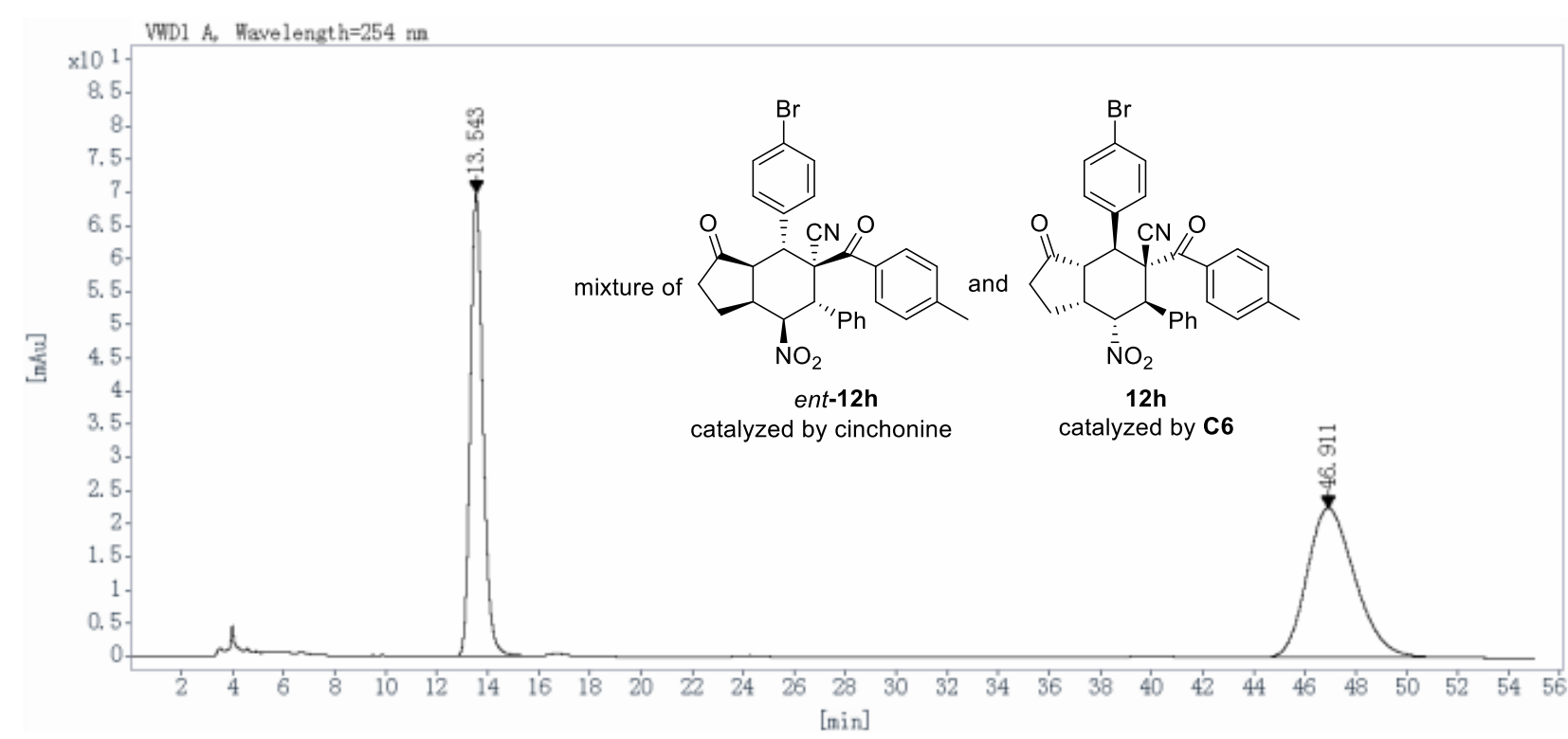

\begin{tabular}{cccccc}
$\begin{array}{c}\text { Ret Time } \\
{[\mathrm{min}]}\end{array}$ & $\begin{array}{c}\text { Peak } \\
\text { Type }\end{array}$ & $\begin{array}{c}\text { Width } \\
{[\mathrm{min}]}\end{array}$ & $\begin{array}{c}\text { Height } \\
{[\mathrm{mAU}]}\end{array}$ & $\begin{array}{c}\text { Area } \\
{\left[\mathrm{mAU}^{*} \mathbf{s}\right]}\end{array}$ & $\begin{array}{c}\text { Area } \\
{[\%]}\end{array}$ \\
\hline 13.543 & BB & 0.53 & 69.7607 & 2395.4045 & 45.0591 \\
46.911 & BB & 1.81 & 22.4417 & 2920.7400 & 54.9409 \\
& & & Totals: & 5316.1445 & 100.0000
\end{tabular}

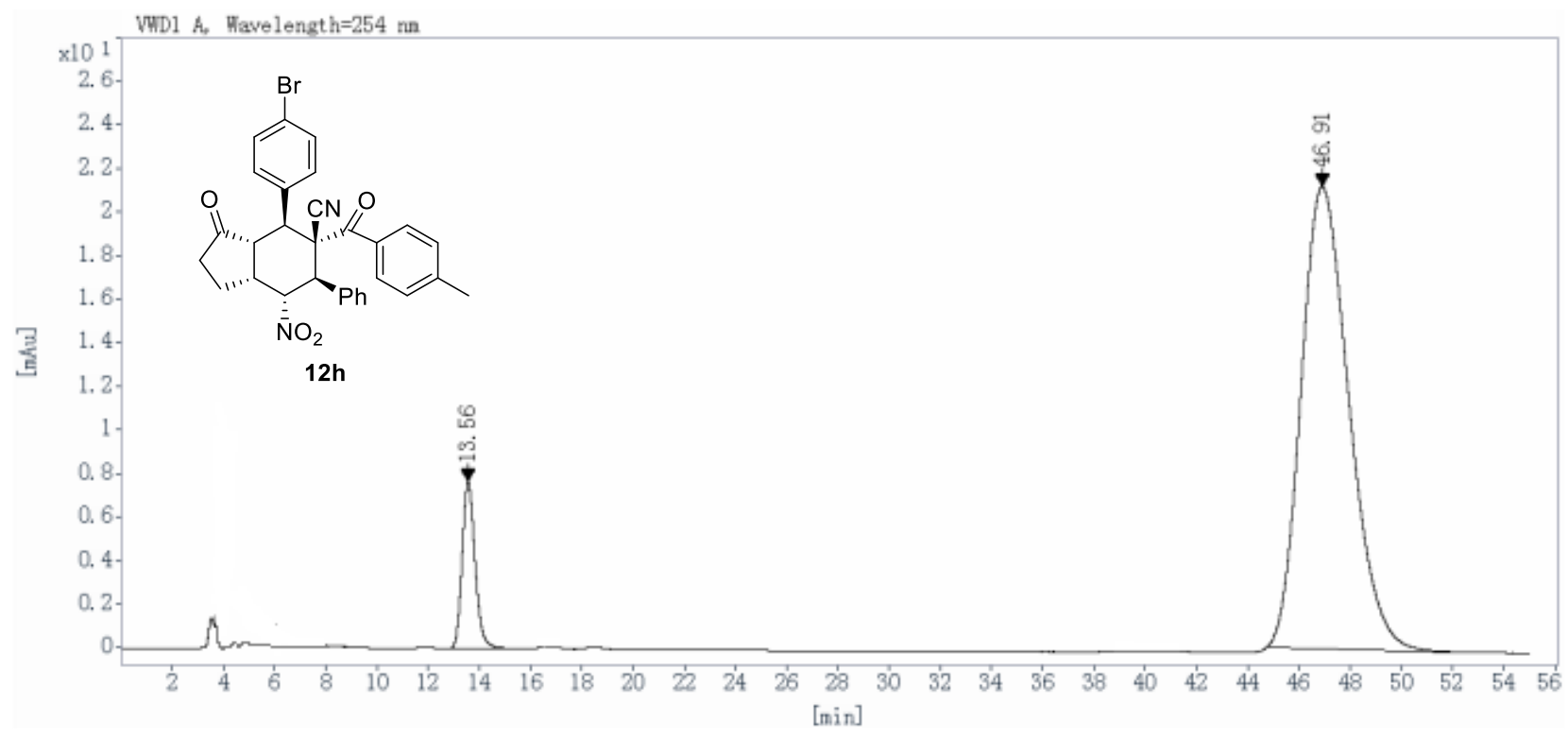

$\begin{array}{cccccc}\begin{array}{c}\text { Ret Time } \\ {[\mathrm{min}]}\end{array} & \begin{array}{c}\text { Peak } \\ \text { Type }\end{array} & \begin{array}{c}\text { Width } \\ {[\mathrm{min}]}\end{array} & \begin{array}{c}\text { Height } \\ {[\mathrm{mAU}]}\end{array} & \begin{array}{c}\text { Area } \\ {\left[\mathrm{mAU} \mathbf{U}^{*}\right]}\end{array} & \begin{array}{c}\text { Area } \\ {[\%]}\end{array} \\ 13.560 & \text { BB } & 0.54 & 7.6879 & 269.0080 & 8.9206 \\ 46.910 & \text { BB } & 1.79 & \begin{array}{c}21.1874 \\ \text { Totals: }\end{array} & 2746.5793 & 91.0794 \\ & & & & & \end{array}$




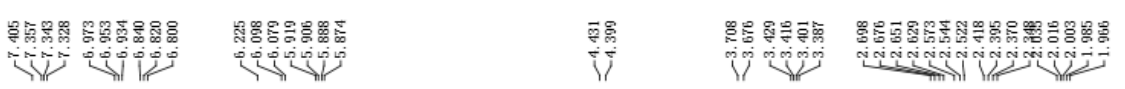

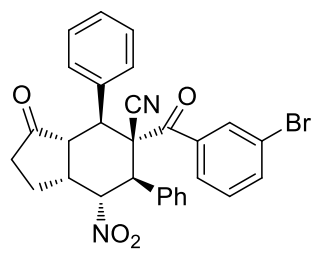

12

$\left(400 \mathrm{MHz}, \mathrm{CDCl}_{3}\right)$

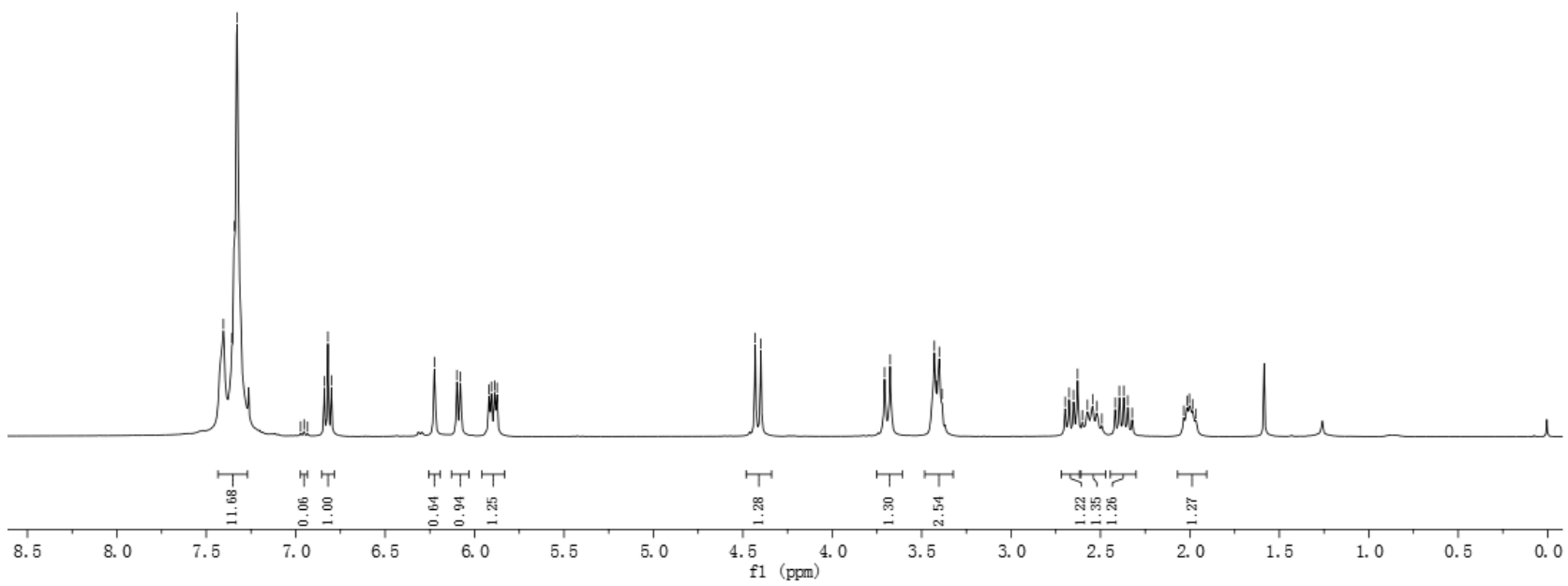

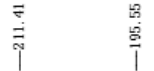

g

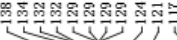

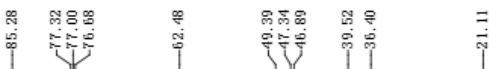

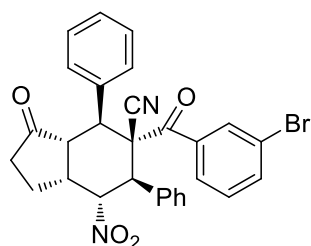

12i

$\left(100 \mathrm{MHz}, \mathrm{CDCl}_{3}\right)$

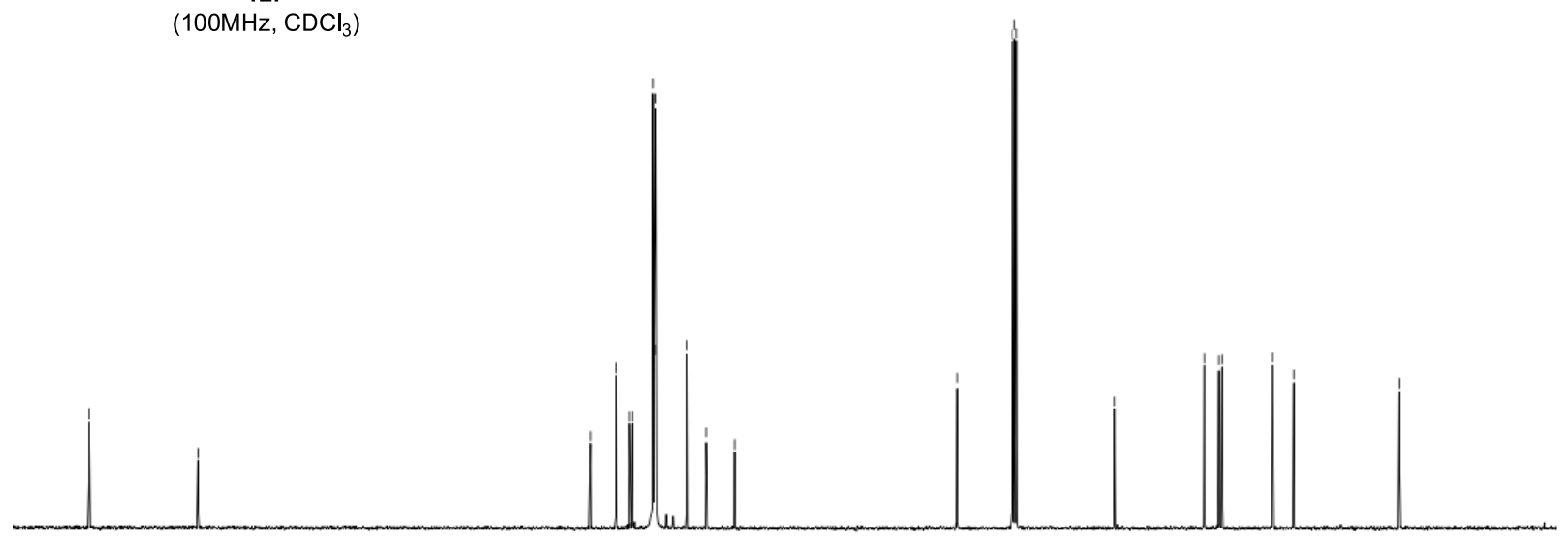

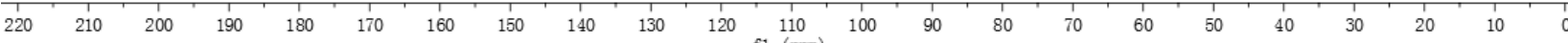




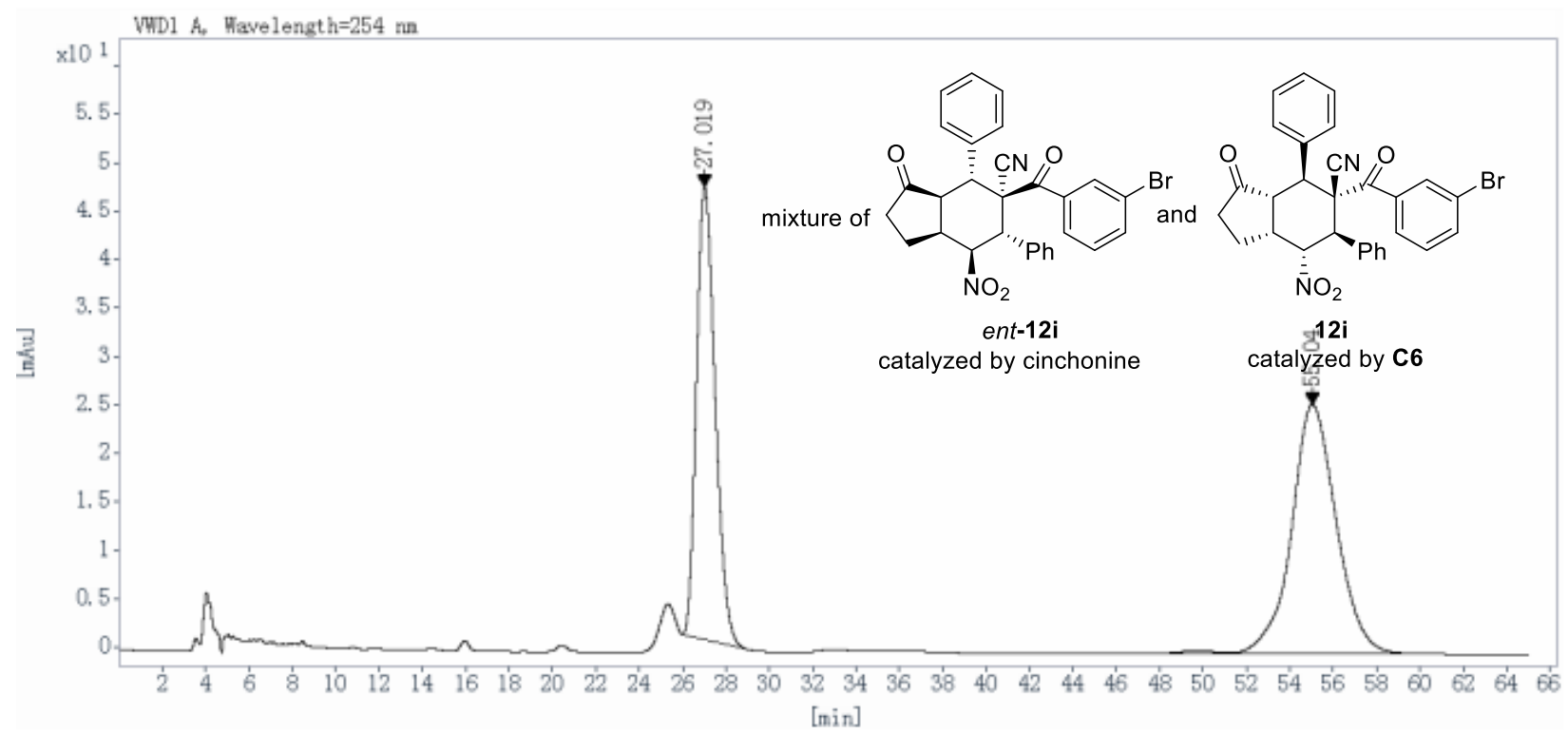

\begin{tabular}{cccccc}
$\begin{array}{c}\text { Ret Time } \\
\text { [min] }\end{array}$ & $\begin{array}{c}\text { Peak } \\
\text { Type }\end{array}$ & $\begin{array}{c}\text { Width } \\
{[\mathrm{min}]}\end{array}$ & $\begin{array}{c}\text { Height } \\
{[\mathrm{mAU}]}\end{array}$ & $\begin{array}{c}\text { Area } \\
{\left[\mathrm{mAU}^{*} \text { s] }\right.}\end{array}$ & $\begin{array}{c}\text { Area } \\
{[\%]}\end{array}$ \\
\hline 27.019 & BB & 0.93 & 46.6330 & 2809.3738 & 42.8499 \\
55.040 & VV R & 2.14 & 25.7241 & 3746.9434 & 57.1501 \\
& & & Totals: & 6556.3171 & 100.0000
\end{tabular}

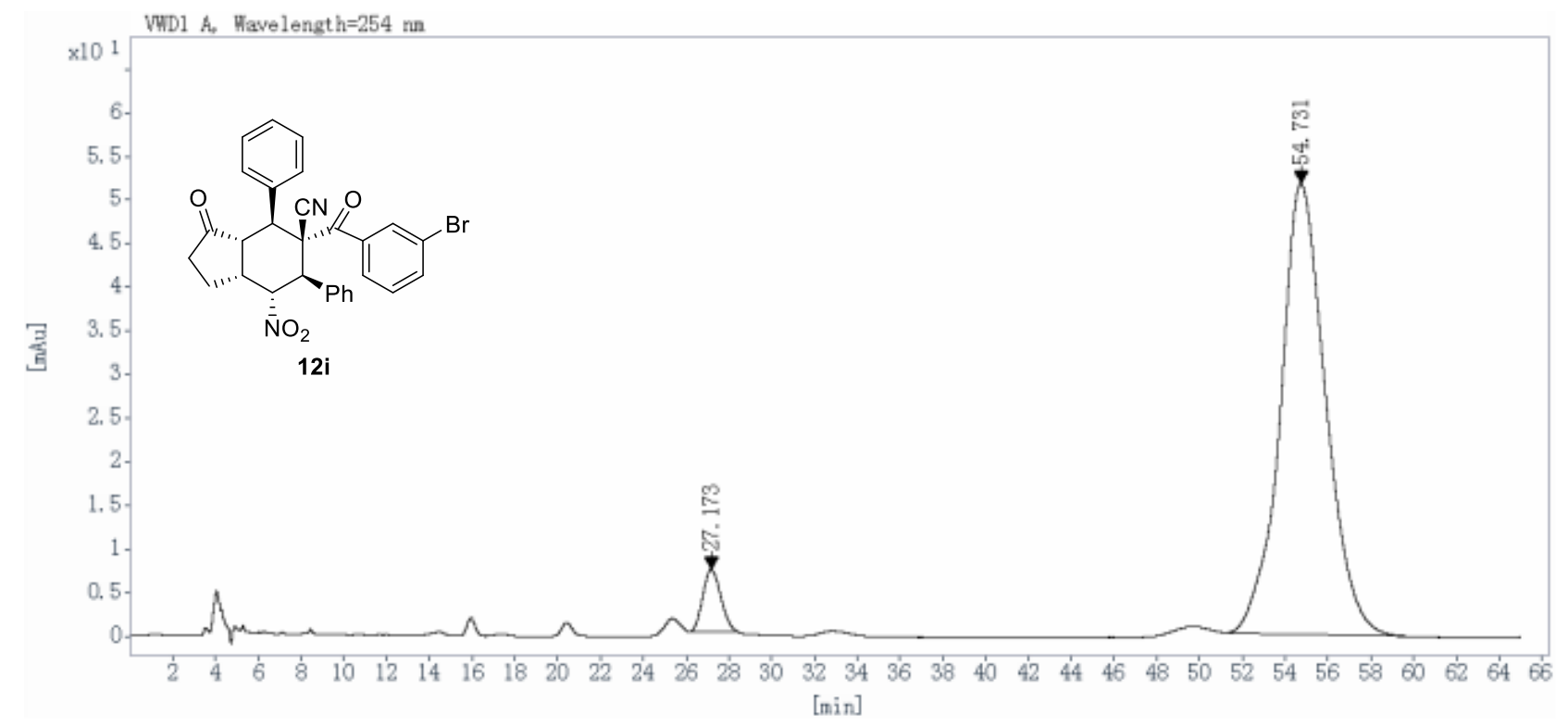

\begin{tabular}{cccccc}
$\begin{array}{c}\text { Ret Time } \\
{[\mathrm{min}]}\end{array}$ & $\begin{array}{c}\text { Peak } \\
\text { Type }\end{array}$ & $\begin{array}{c}\text { Width } \\
{[\mathrm{min}]}\end{array}$ & $\begin{array}{c}\text { Height } \\
{[\mathrm{mAU}]}\end{array}$ & $\begin{array}{c}\text { Area } \\
{\left[\mathrm{mAU}^{*} \mathrm{~s}\right]}\end{array}$ & $\begin{array}{c}\text { Area } \\
{[\%]}\end{array}$ \\
\hline 27.173 & BBA & 0.91 & 7.0865 & 413.7141 & 5.1486 \\
54.731 & BB & 2.17 & 51.6717 & 7621.7832 & 94.8514 \\
& & & Totals: & 8035.4973 & 100.0000
\end{tabular}




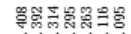

पivivi

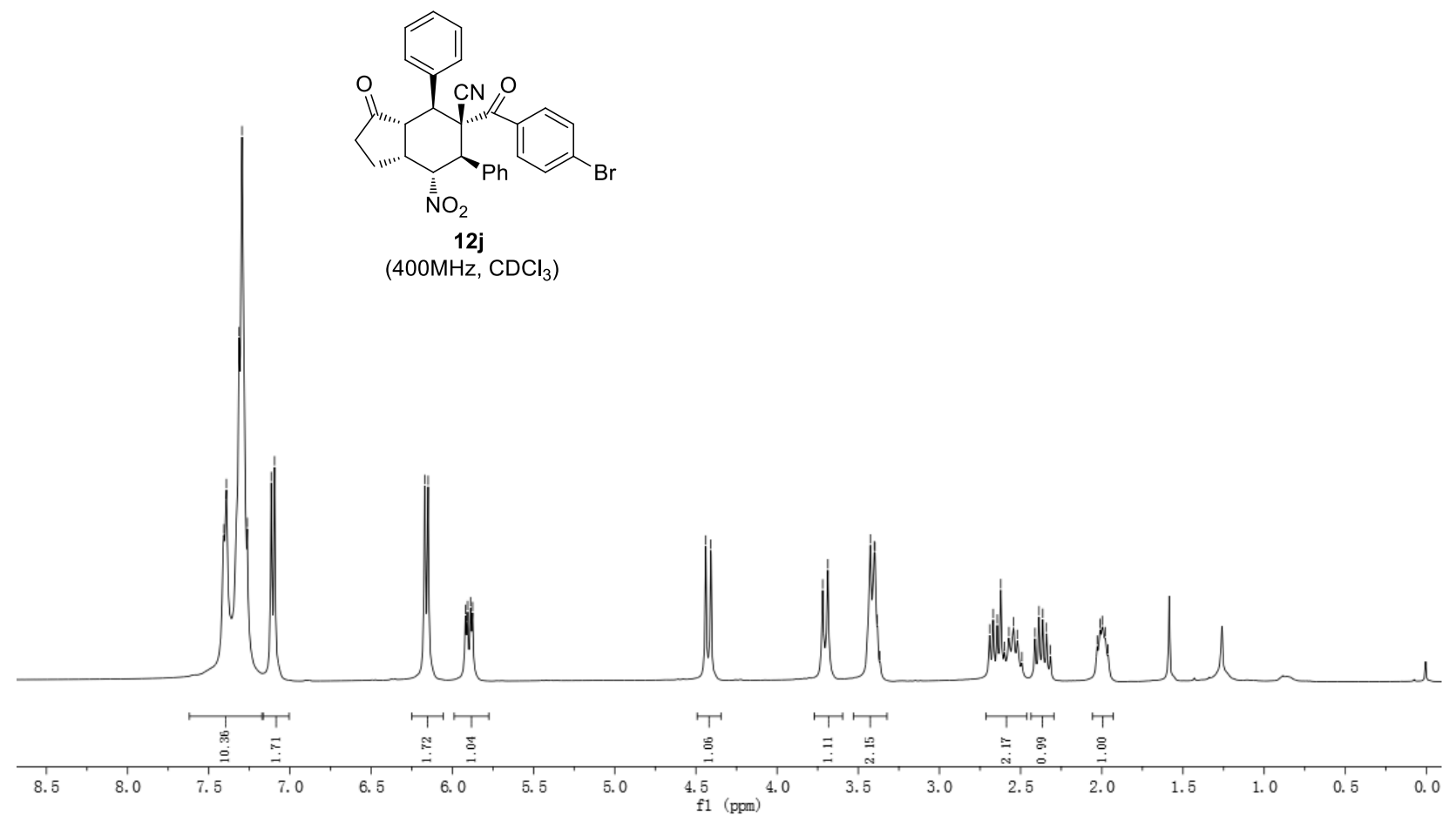

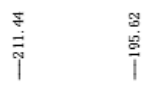

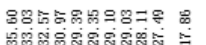

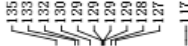

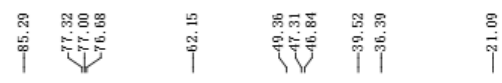

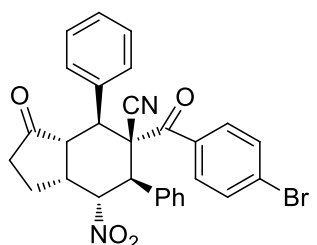

12j

$\left(100 \mathrm{MHz}, \mathrm{CDCl}_{3}\right)$

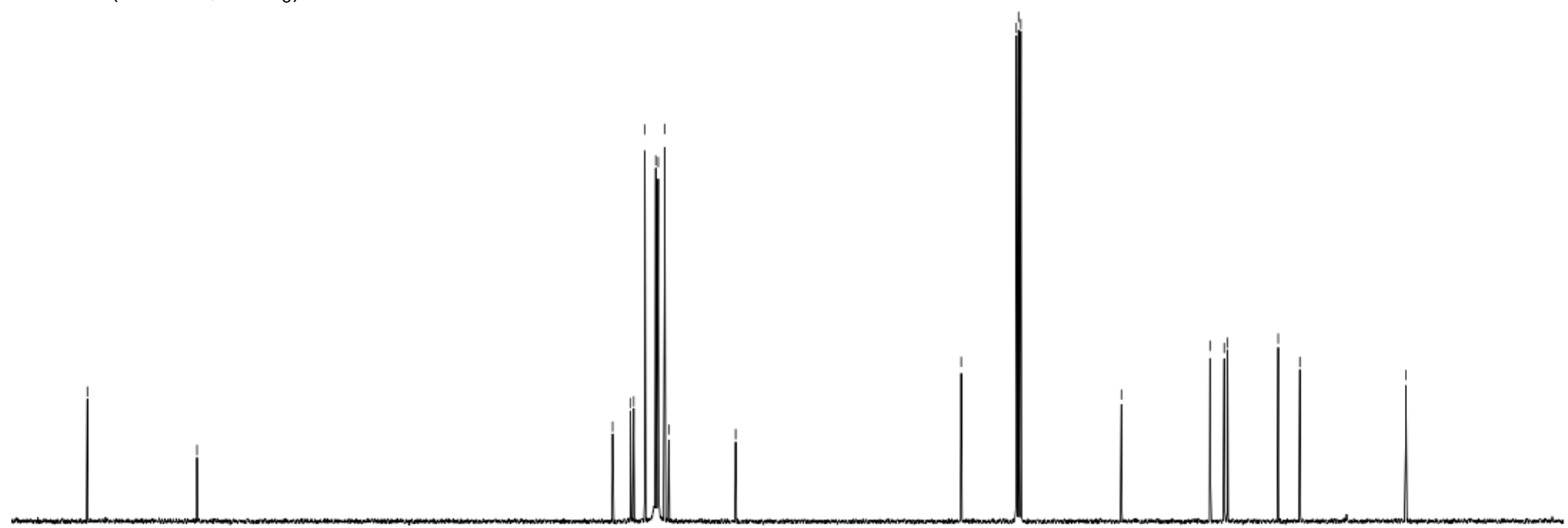

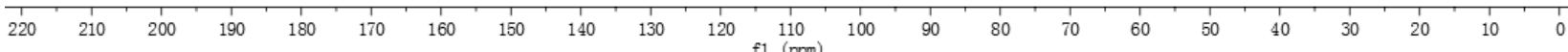




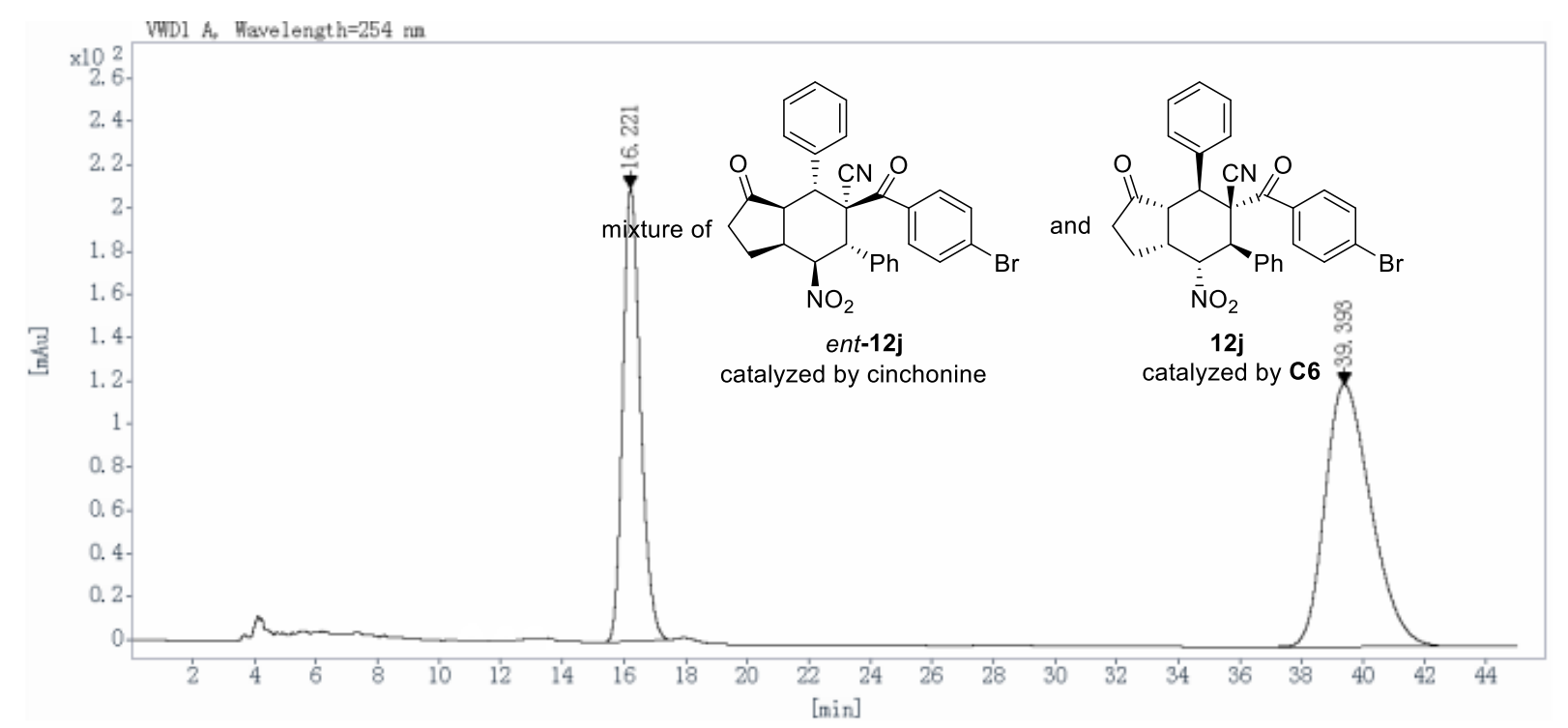

\begin{tabular}{|c|c|c|c|c|c|}
\hline $\begin{array}{l}\text { Ret Time } \\
\text { [min] }\end{array}$ & $\begin{array}{l}\text { Peak } \\
\text { Type }\end{array}$ & $\begin{array}{l}\text { Width } \\
\text { [min] }\end{array}$ & $\begin{array}{l}\text { Height } \\
{[\mathbf{m A U}]}\end{array}$ & $\begin{array}{c}\text { Area } \\
{\left[\mathbf{m A U}^{*} \mathbf{s}\right]}\end{array}$ & $\begin{array}{l}\text { Area } \\
{[\%]}\end{array}$ \\
\hline 16.221 & BB & 0.62 & 209.6729 & 8408.2002 & 40.0232 \\
\hline 39.393 & BB & 1.59 & 121.4745 & 12600.0928 & 59.9768 \\
\hline
\end{tabular}

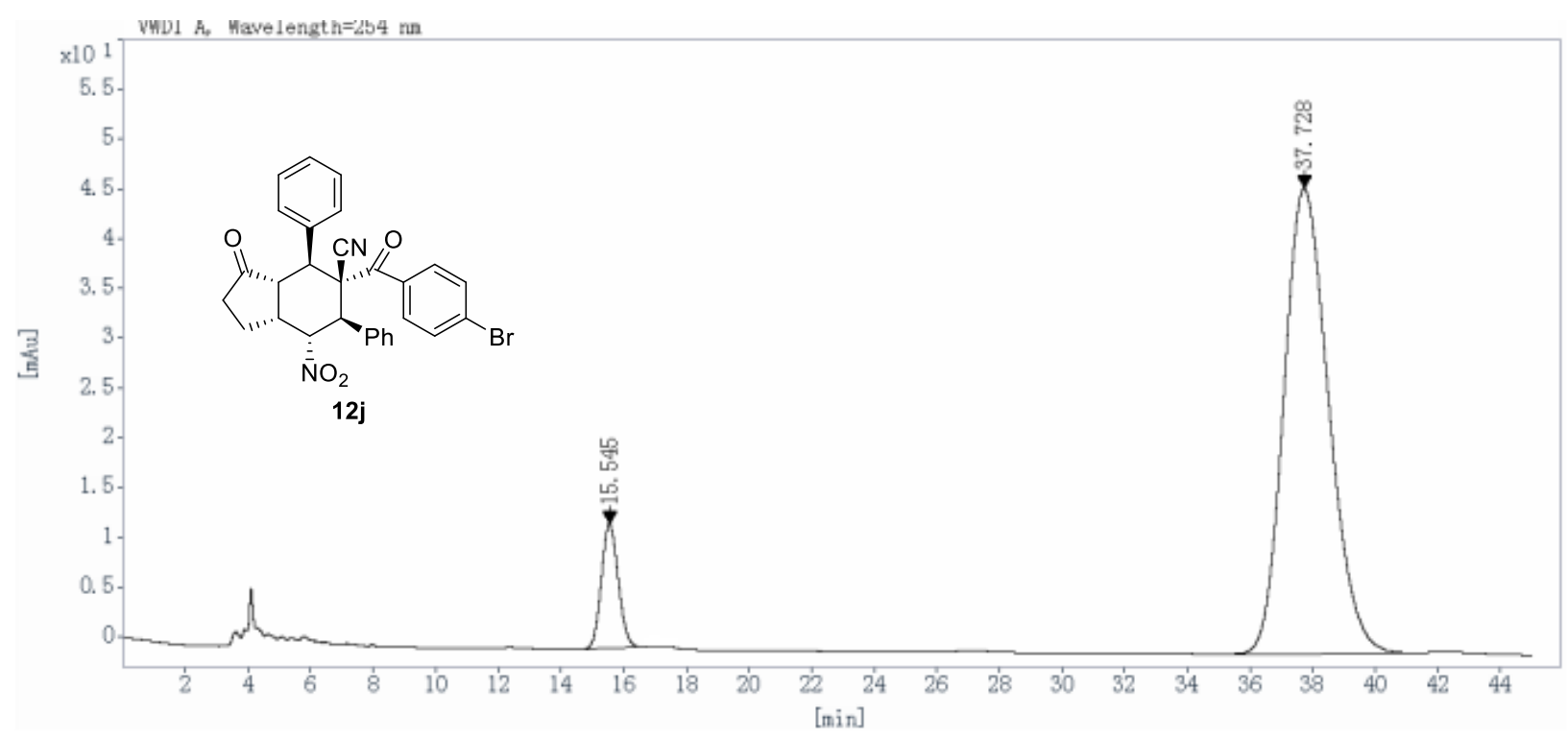

\begin{tabular}{cccccc}
$\begin{array}{c}\text { Ret Time } \\
{[\mathrm{min}]}\end{array}$ & $\begin{array}{c}\text { Peak } \\
\text { Type }\end{array}$ & $\begin{array}{c}\text { Width } \\
{[\mathrm{min}]}\end{array}$ & $\begin{array}{c}\text { Height } \\
{[\mathrm{mAU}]}\end{array}$ & $\begin{array}{c}\text { Area } \\
{[\mathrm{mAU} \text { s] }}\end{array}$ & $\begin{array}{c}\text { Area } \\
{[\%]}\end{array}$ \\
\hline 15.545 & BB & 0.58 & 12.5185 & 468.5338 & 9.1841 \\
37.728 & BB & 1.53 & 46.6987 & 4633.0532 & 90.8159 \\
& & & Totals: & 5101.5871 & 100.0000
\end{tabular}




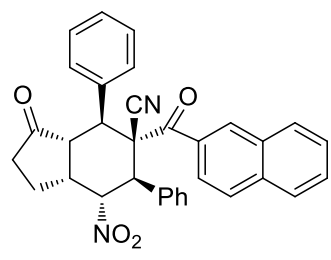

12k

$\left(400 \mathrm{MHz}, \mathrm{CDCl}_{3}\right)$

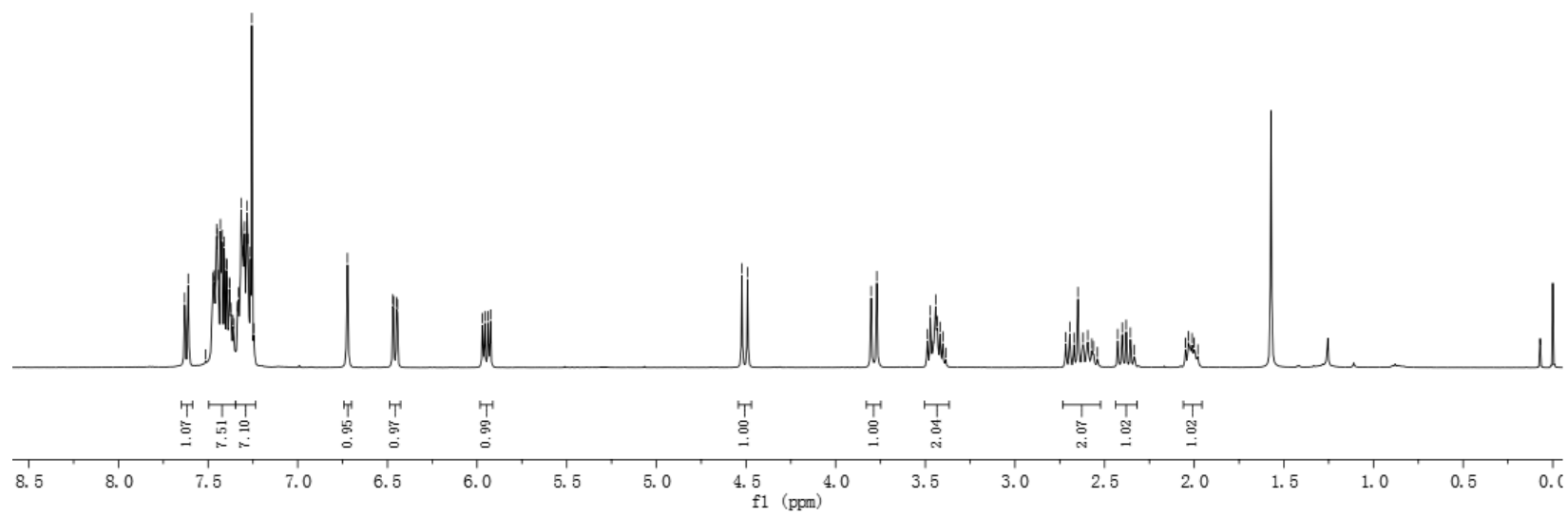

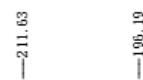

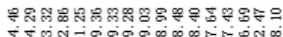

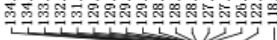

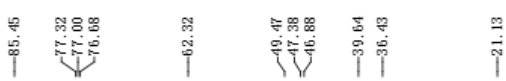

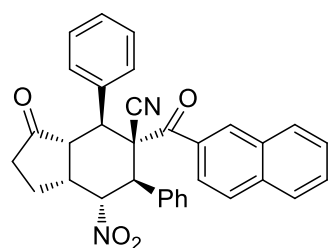

$12 \mathrm{k}$

$\left(100 \mathrm{MHz}, \mathrm{CDCl}_{3}\right)$

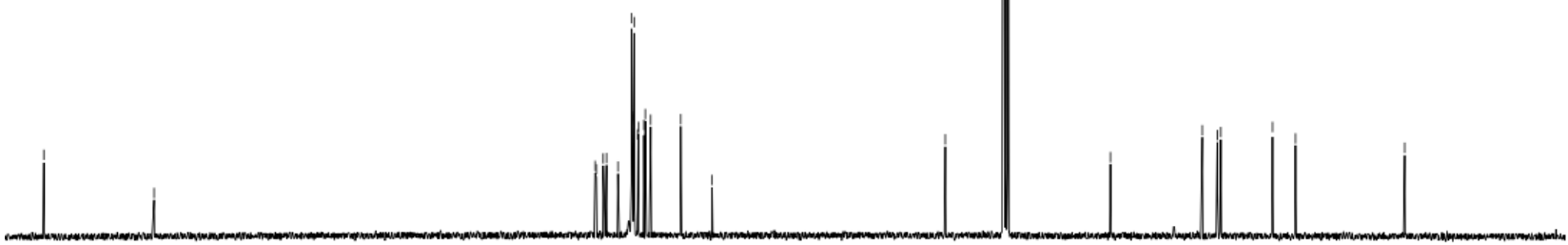




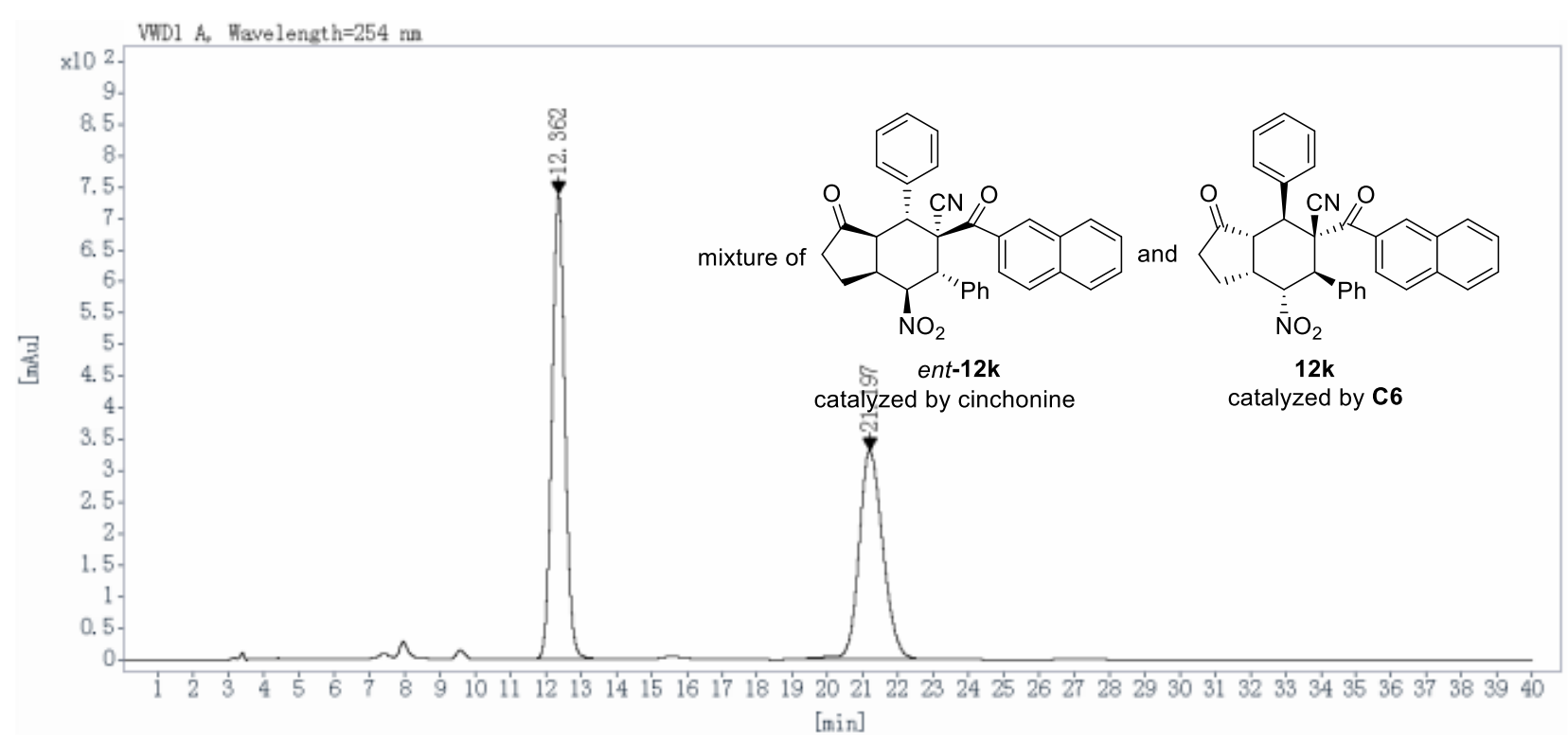

$\begin{array}{cccccc}\begin{array}{c}\text { Ret Time } \\ \text { [min] }\end{array} & \begin{array}{c}\text { Peak } \\ \text { Type }\end{array} & \begin{array}{c}\text { Width } \\ {[\mathrm{min}]}\end{array} & \begin{array}{c}\text { Height } \\ {[\mathrm{mAU}]}\end{array} & \begin{array}{c}\text { Area } \\ {[\mathrm{mAU} \text { *s] }}\end{array} & \begin{array}{c}\text { Area } \\ {[\%]}\end{array} \\ 12.362 & \text { BB } & 0.40 & 737.8484 & 18788.9141 & 54.6366 \\ 21.197 & \text { BB } & 0.73 & 330.5725 & 15599.9814 & 45.3634 \\ & & & \text { Totals: } & 34388.8955 & 100.0000\end{array}$

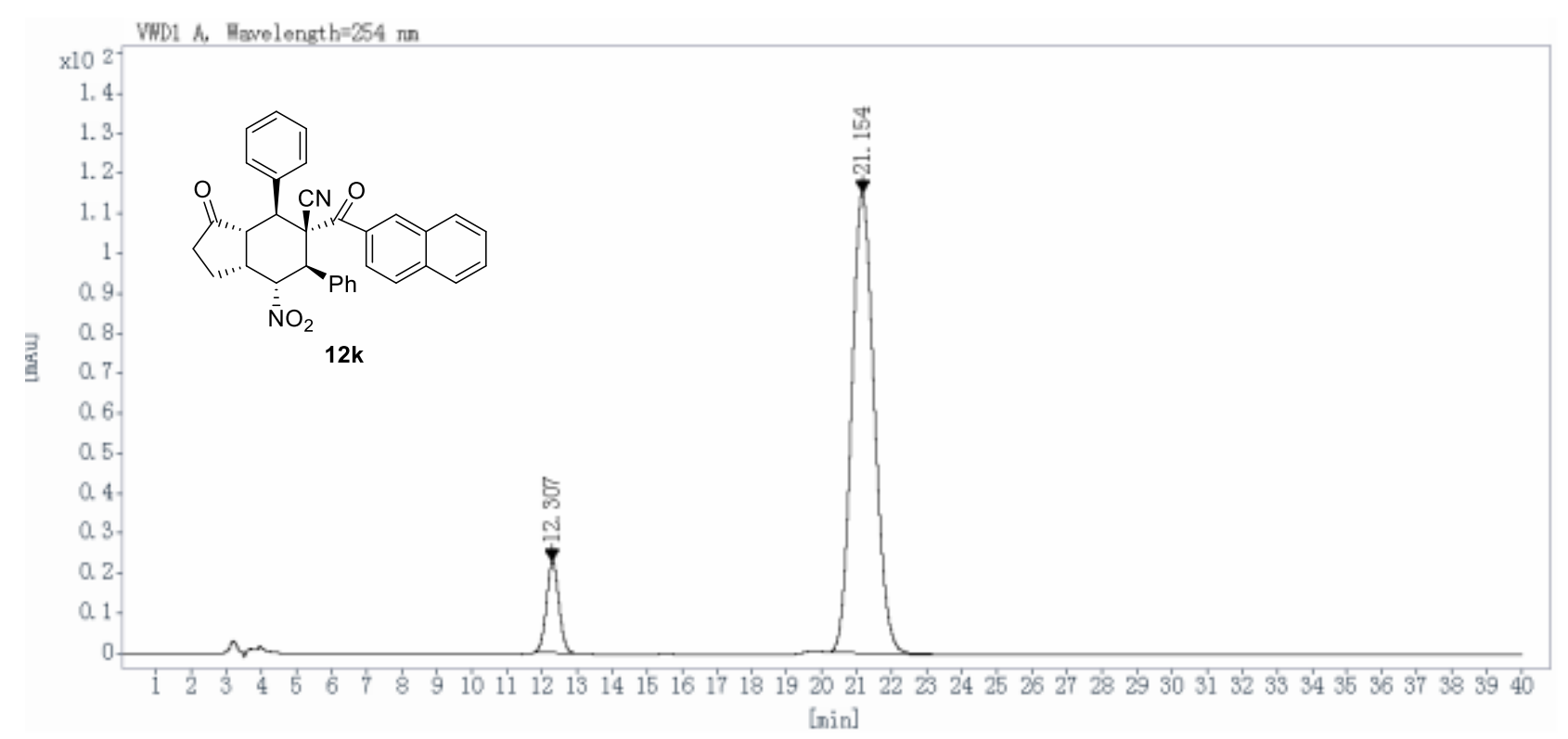

\begin{tabular}{cccccc}
$\begin{array}{c}\text { Ret Time } \\
\text { [min] }\end{array}$ & $\begin{array}{c}\text { Peak } \\
\text { Type }\end{array}$ & $\begin{array}{c}\text { Width } \\
{[\mathrm{min}]}\end{array}$ & $\begin{array}{c}\text { Height } \\
{[\mathrm{mAU} \text { ] }}\end{array}$ & $\begin{array}{c}\text { Area } \\
{[\mathrm{mAU} \text { *s] }}\end{array}$ & $\begin{array}{c}\text { Area } \\
{[\%]}\end{array}$ \\
\hline 12.307 & BBA & 0.38 & 22.7717 & 565.5047 & 9.8843 \\
21.154 & BB & 0.70 & 114.8843 & 5155.7139 & 90.1157 \\
& & & Totals: & 5721.2186 & 100.0000
\end{tabular}




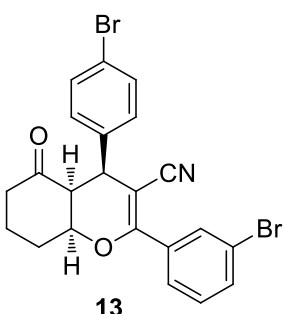

$\left(400 \mathrm{MHz}, \mathrm{CDCl}_{3}\right)$
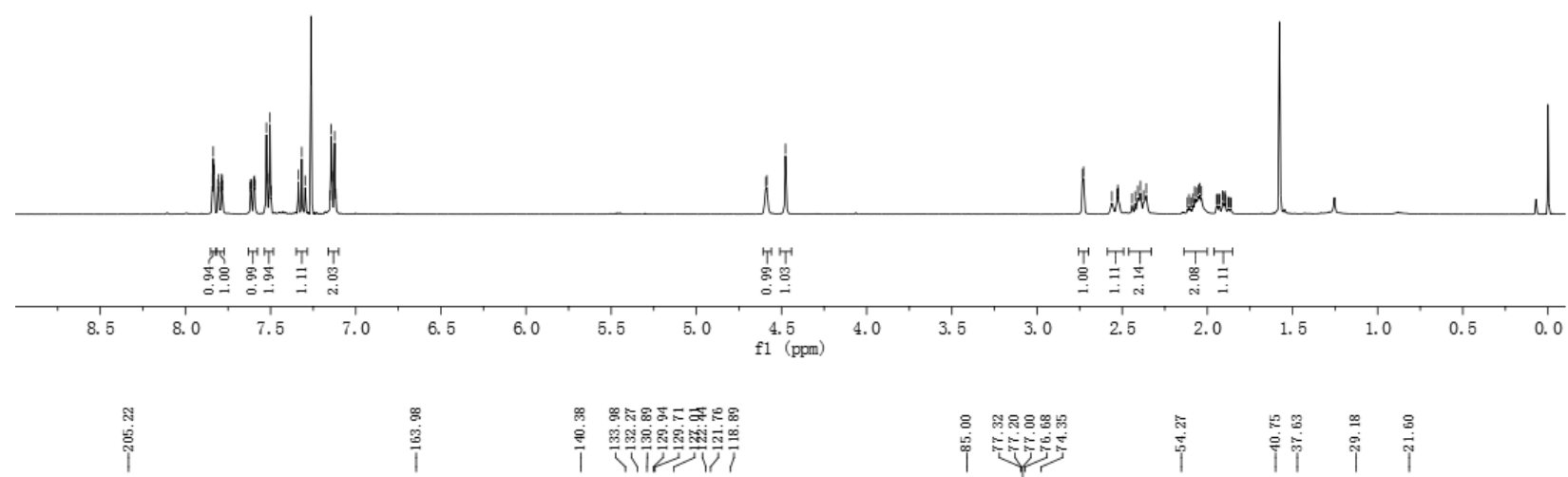

i

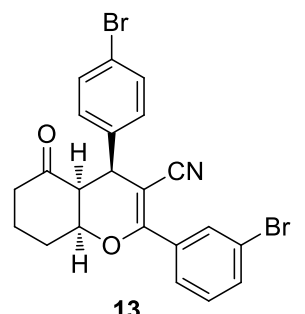

$\left(100 \mathrm{MHz}, \mathrm{CDCl}_{3}\right)$

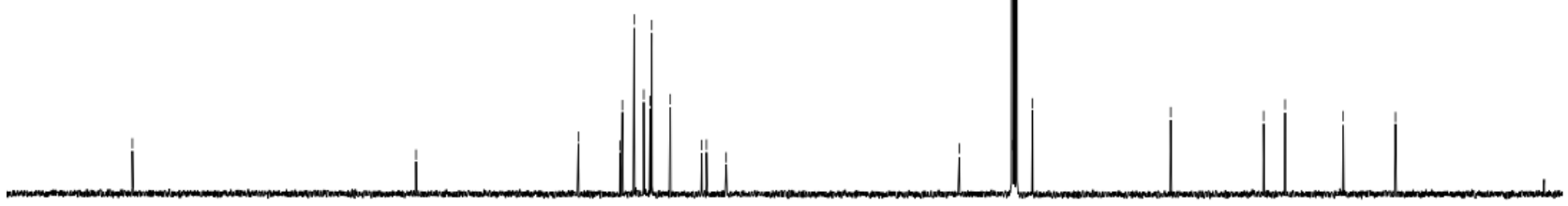

$\begin{array}{llllllllllllllllllllllllllll}220 & 210 & 200 & 190 & 180 & 170 & 160 & 150 & 140 & 130 & 120 & 110 & 100 & 90 & 80 & 70 & 60 & 50 & 40 & 30 & 20 & 10 & 0\end{array}$ 


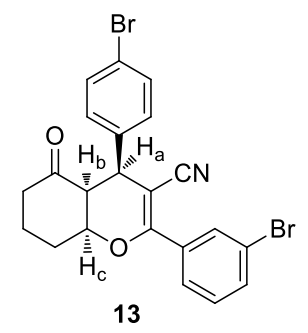

${ }^{1} \mathrm{H}$ NMR $\left(400 \mathrm{MHz}, \mathrm{CDCl}_{3}\right)$ ${ }^{13} \mathrm{C} \mathrm{NMR} \mathrm{(100MHz,} \mathrm{CDCl}_{3}$ )

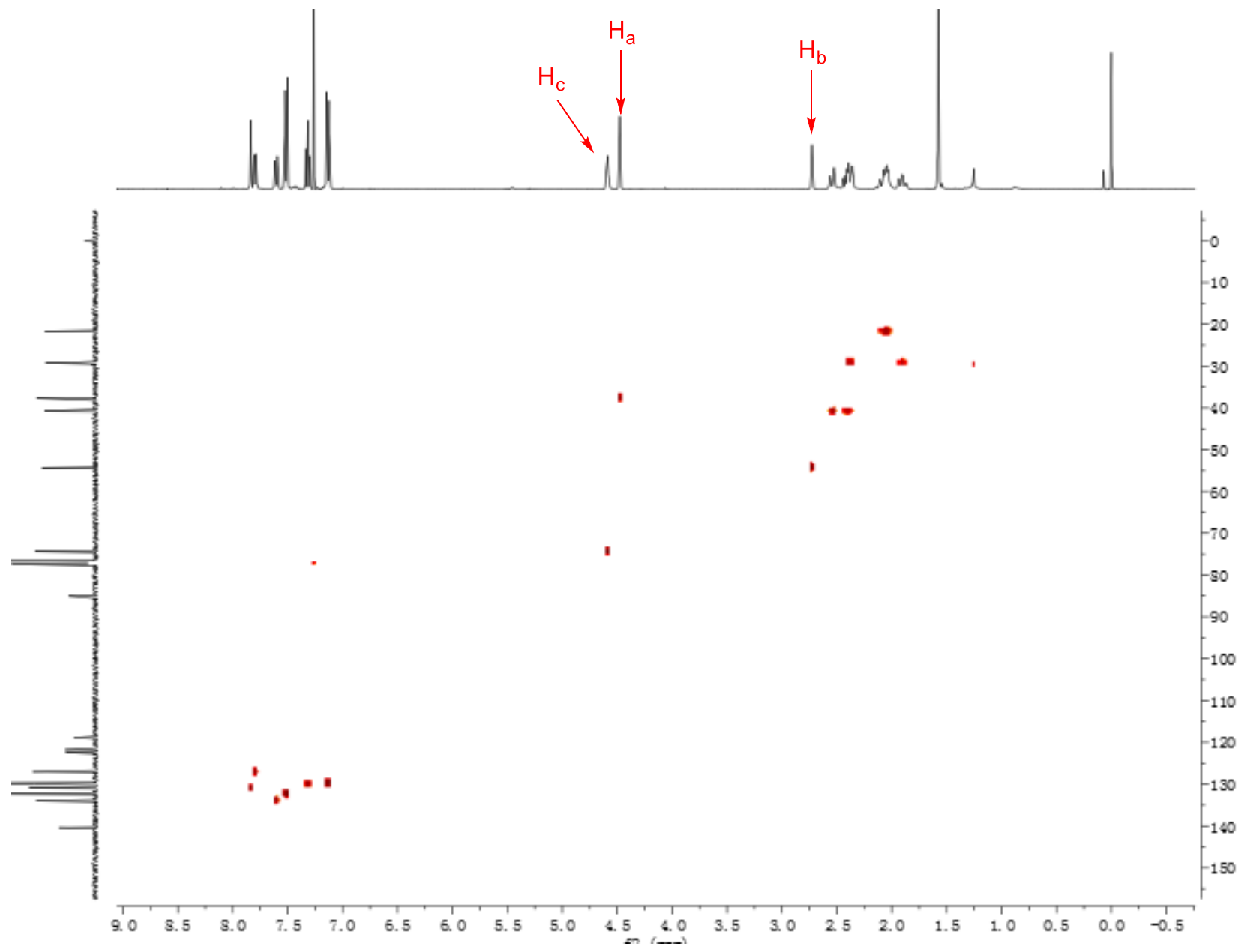

S128 


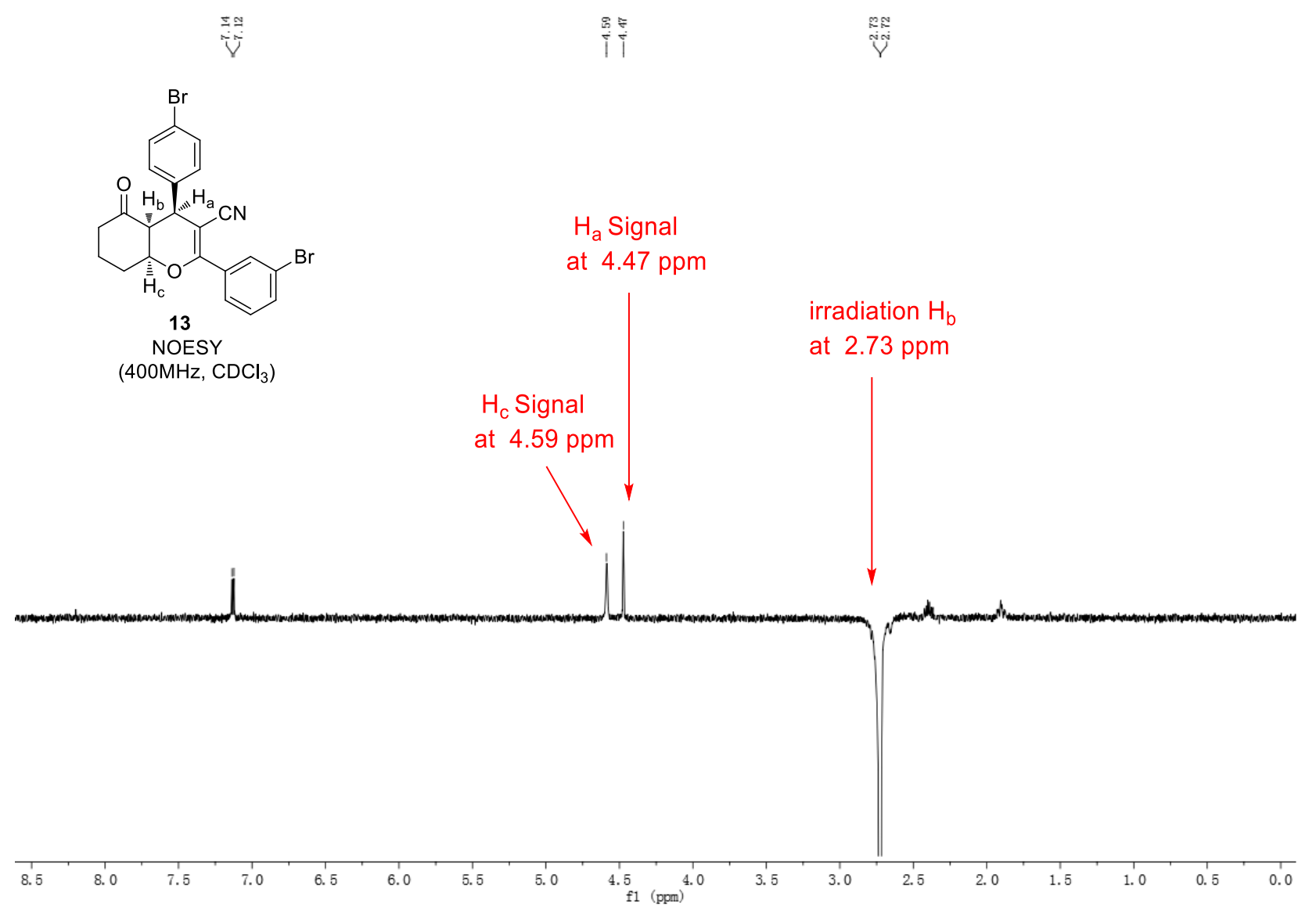




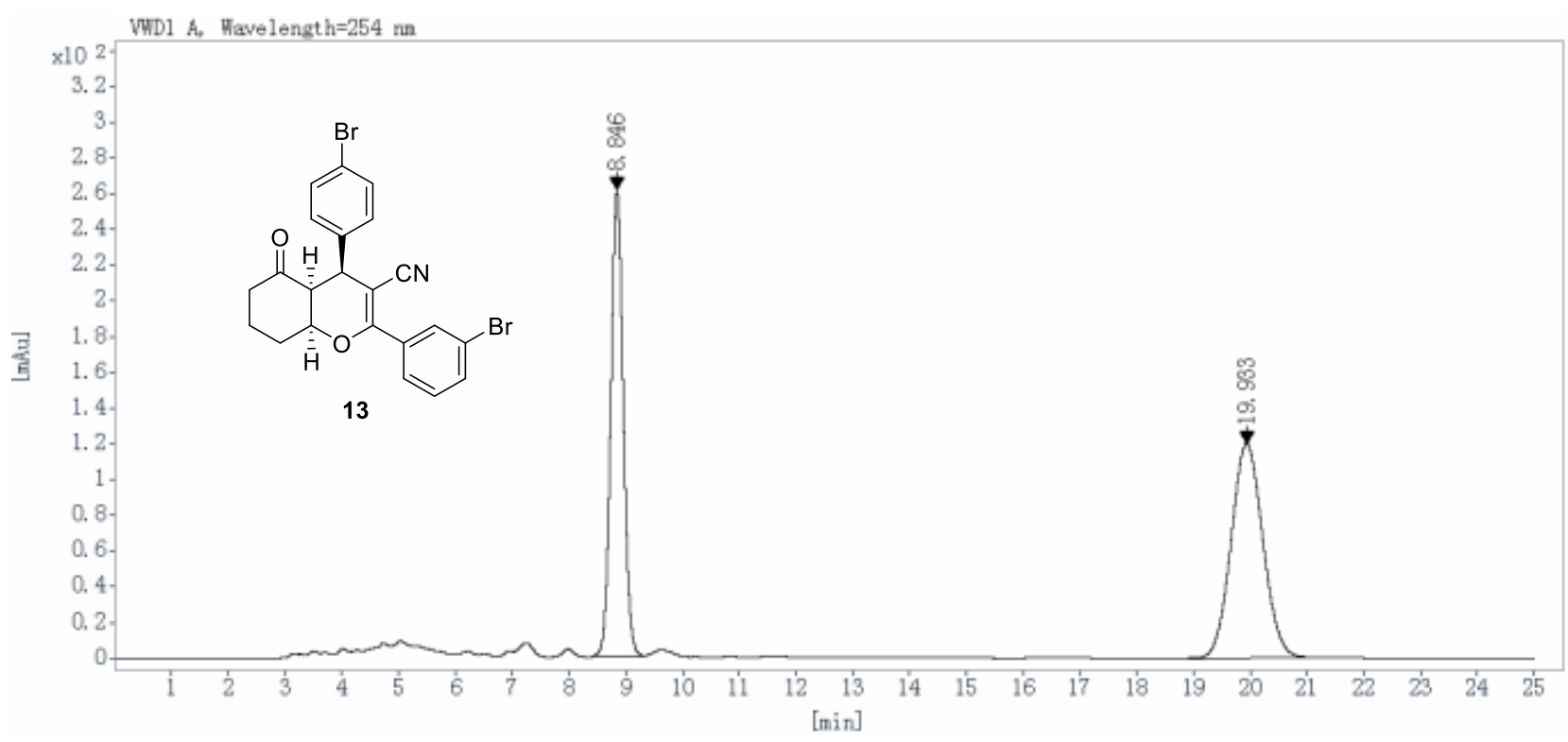

\begin{tabular}{|c|c|c|c|c|c|}
\hline $\begin{array}{l}\text { Ret Time } \\
\text { [min] }\end{array}$ & $\begin{array}{l}\text { Peak } \\
\text { Type }\end{array}$ & $\begin{array}{l}\text { Width } \\
\text { [min] }\end{array}$ & $\begin{array}{l}\text { Height } \\
{[\mathbf{m A U}]}\end{array}$ & $\begin{array}{c}\text { Area } \\
{\left[\mathrm{mAU}^{*} \mathbf{s}\right]}\end{array}$ & $\begin{array}{l}\text { Area } \\
{[\%]}\end{array}$ \\
\hline 8.846 & BB & 0.24 & 260.5586 & 3960.1890 & 46.0579 \\
\hline 19.933 & BB & 0.60 & 119.8027 & 4638.1021 & 53.9421 \\
\hline & & & Totals: & 8598.2910 & 100.0000 \\
\hline
\end{tabular}

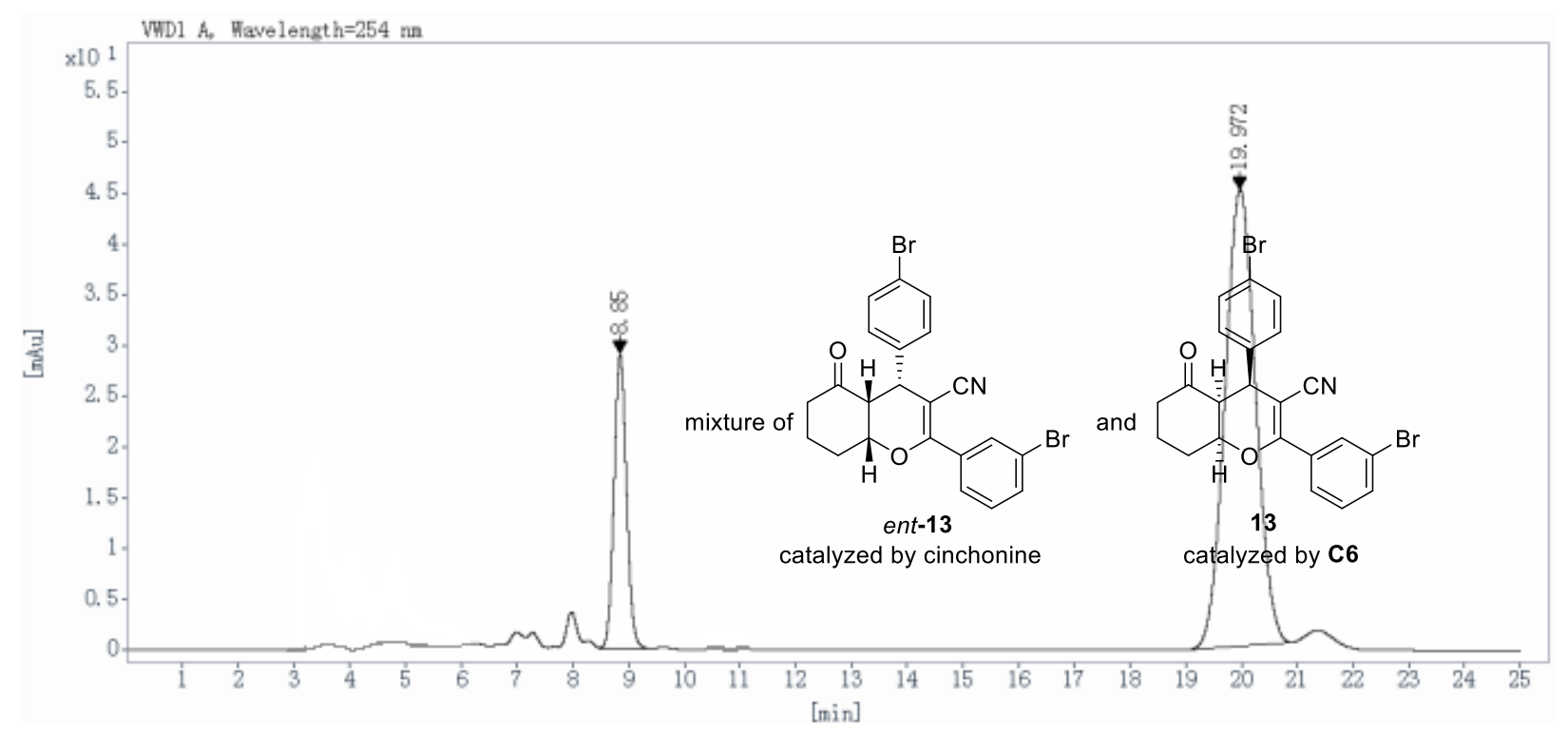

$\begin{array}{cccccc}\begin{array}{c}\text { Ret Time } \\ {[\mathrm{min}]}\end{array} & \begin{array}{c}\text { Peak } \\ \text { Type }\end{array} & \begin{array}{c}\text { Width } \\ {[\mathrm{min}]}\end{array} & \begin{array}{c}\text { Height } \\ {[\mathrm{mAU}]}\end{array} & \begin{array}{c}\text { Area } \\ {\left[\mathrm{mAU}^{*} \mathbf{s}\right]}\end{array} & \begin{array}{c}\text { Area } \\ {[\%]}\end{array} \\ 8.850 & \text { BB } & 0.24 & 28.9652 & 448.3345 & 20.6686 \\ 19.972 & \text { BB } & 0.60 & 44.9013 & 1720.8241 & 79.3314 \\ & & & \text { Totals: } & 2169.1586 & 100.0000\end{array}$




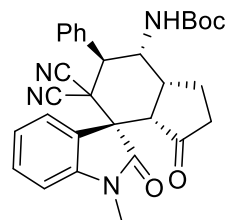

$14\left(400 \mathrm{MHz}, \mathrm{CDCl}_{3}\right)$

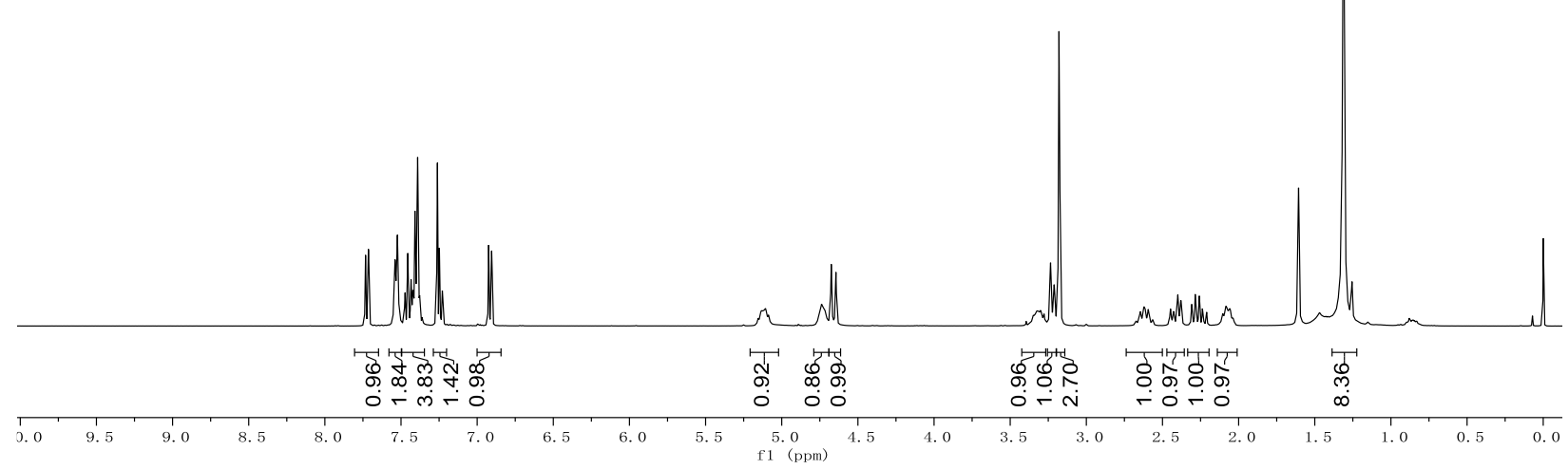

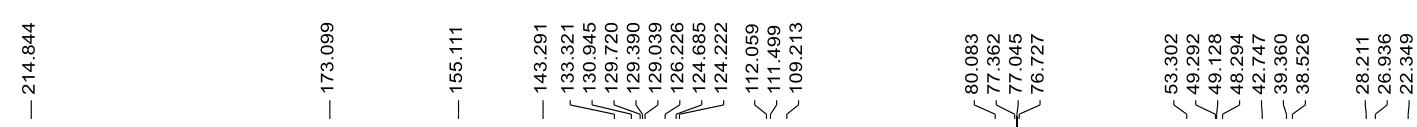

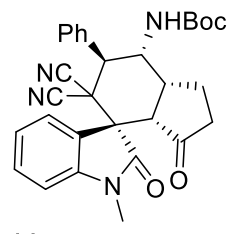

$14\left(100 \mathrm{MHz}, \mathrm{CDCl}_{3}\right)$

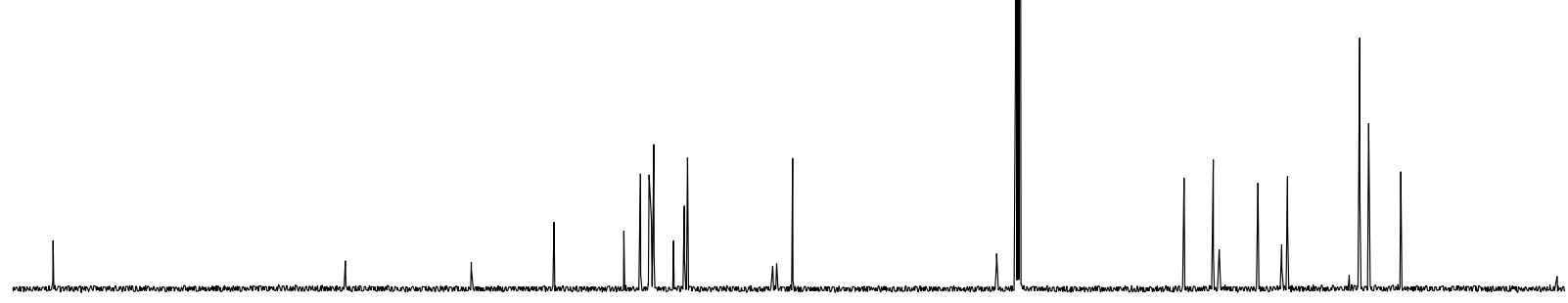




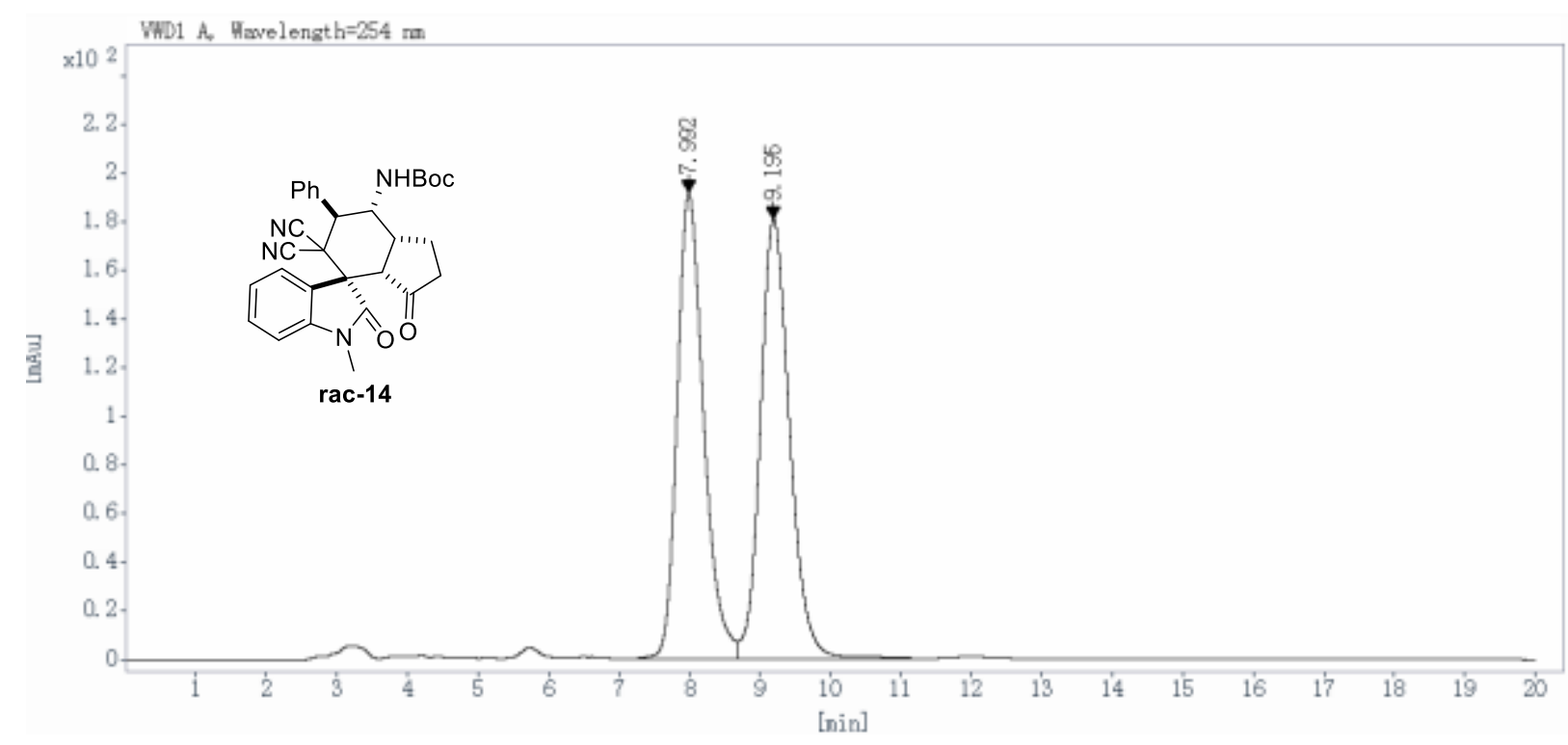

\begin{tabular}{|c|c|c|c|c|c|}
\hline $\begin{array}{l}\text { Ret Time } \\
\text { [min] }\end{array}$ & $\begin{array}{l}\text { Peak } \\
\text { Type }\end{array}$ & $\begin{array}{l}\text { Width } \\
\text { [min] }\end{array}$ & $\begin{array}{l}\text { Height } \\
{[\mathbf{m A U}]}\end{array}$ & $\begin{array}{c}\text { Area } \\
{\left[\mathbf{m A U ^ { * } \mathbf { s } ]}\right.}\end{array}$ & $\begin{array}{c}\text { Area } \\
{[\%]}\end{array}$ \\
\hline 7.992 & BV & 0.40 & 191.4076 & 5003.5518 & 48.9300 \\
\hline \multirow[t]{2}{*}{9.195} & VB & 0.44 & 180.8650 & 5222.3867 & 51.0700 \\
\hline & & & Totals: & 10225.9385 & 100.0000 \\
\hline
\end{tabular}

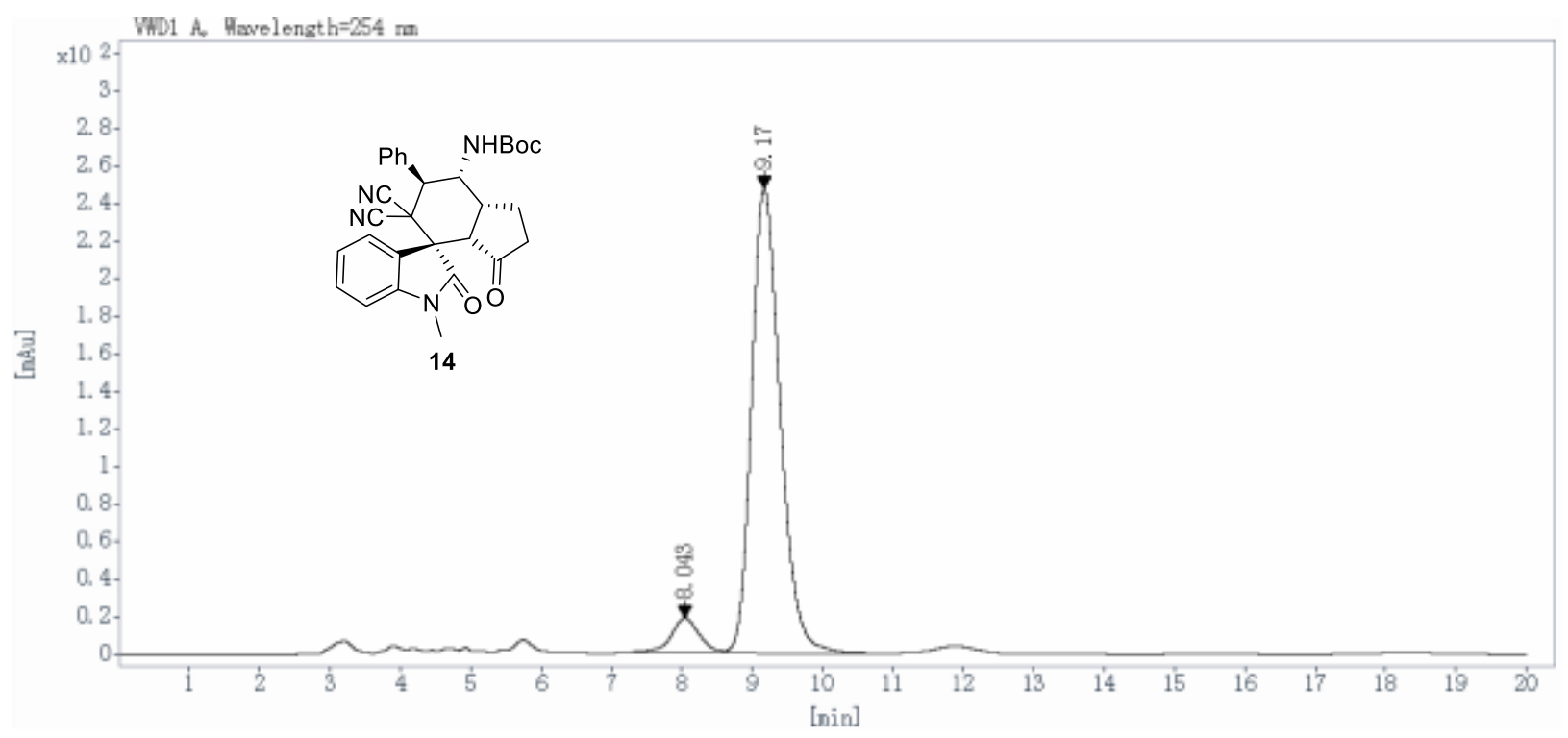

\begin{tabular}{cccccc}
$\begin{array}{c}\text { Ret Time } \\
{[\mathrm{min}]}\end{array}$ & $\begin{array}{c}\text { Peak } \\
\text { Type }\end{array}$ & $\begin{array}{c}\text { Width } \\
{[\mathrm{min}]}\end{array}$ & $\begin{array}{c}\text { Height } \\
{[\mathrm{mAU}]}\end{array}$ & $\begin{array}{c}\text { Area } \\
{[\mathrm{mAU} \text { *s] }}\end{array}$ & $\begin{array}{c}\text { Area } \\
{[\%]}\end{array}$ \\
\hline 8.043 & VV E & 0.42 & 18.4417 & 518.0374 & 6.8868 \\
9.170 & VB R & 0.44 & $\begin{array}{c}247.1892 \\
\text { Totals: }\end{array}$ & 7004.0942 & 93.1132 \\
& & & 7522.1316 & 100.0000
\end{tabular}




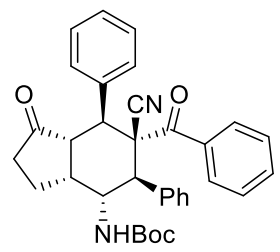

$15\left(100 \mathrm{MHz}, \mathrm{CDCl}_{3}\right)$

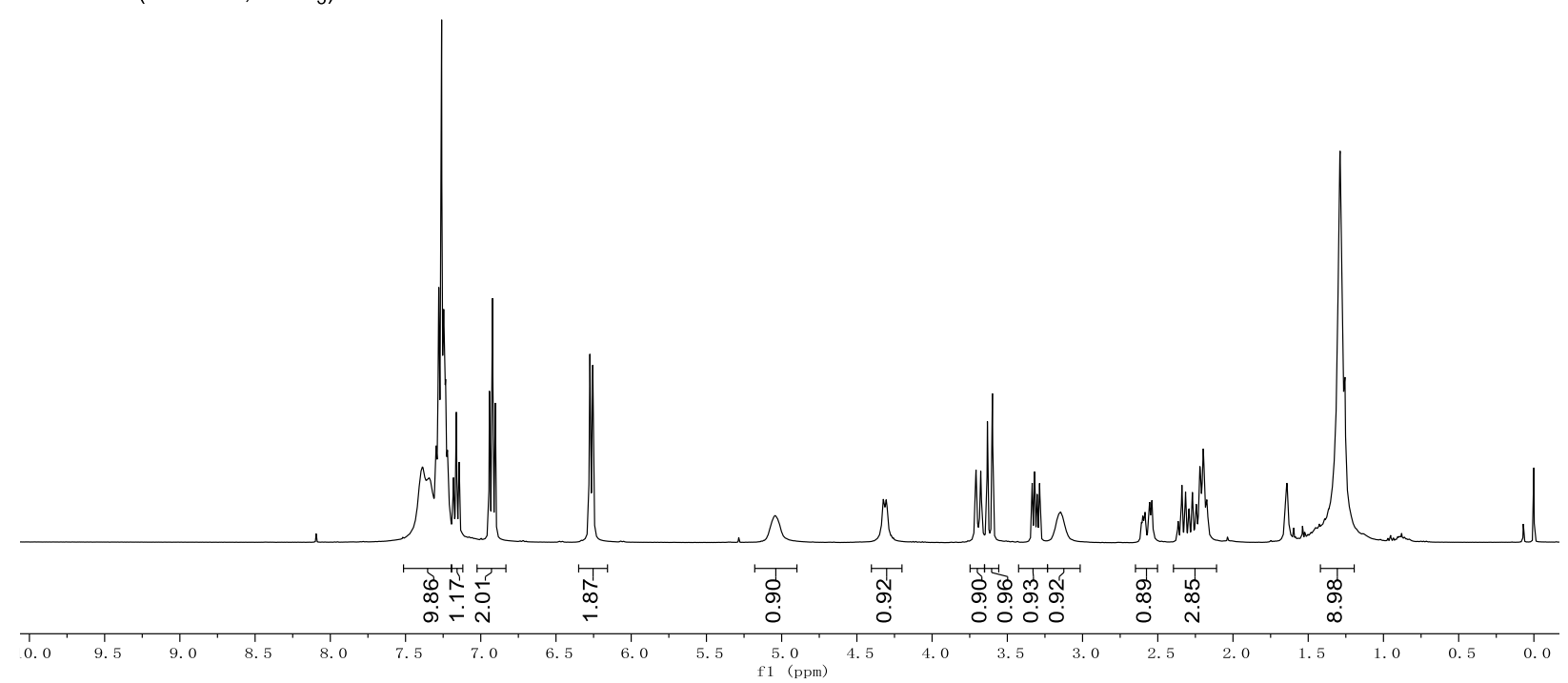

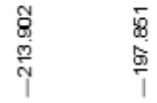

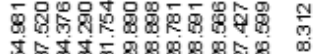

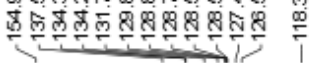

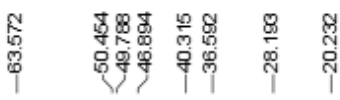

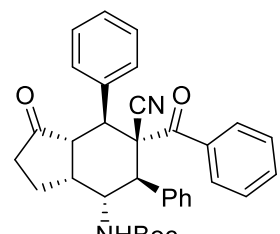

$15\left(400 \mathrm{MHz}, \mathrm{CDCl}_{3}\right)$
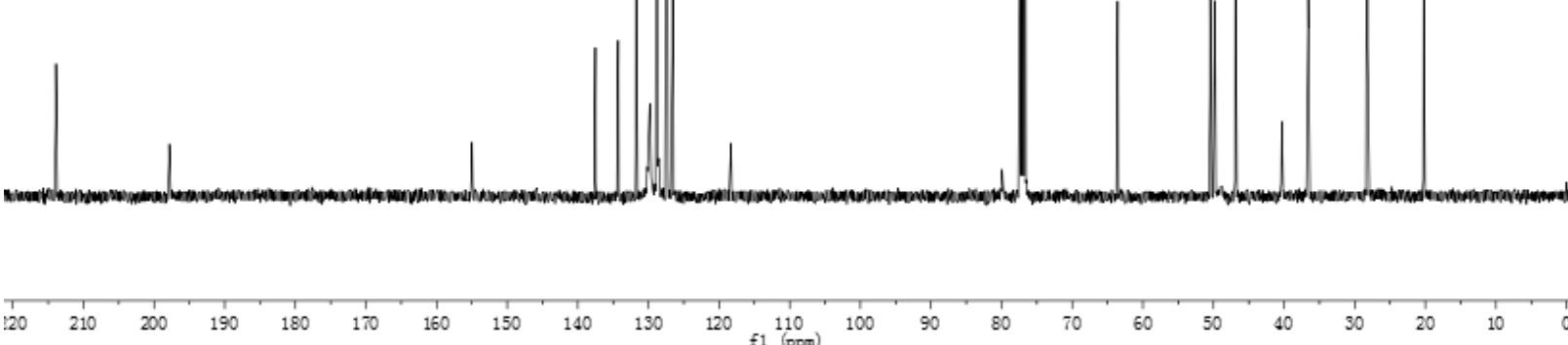


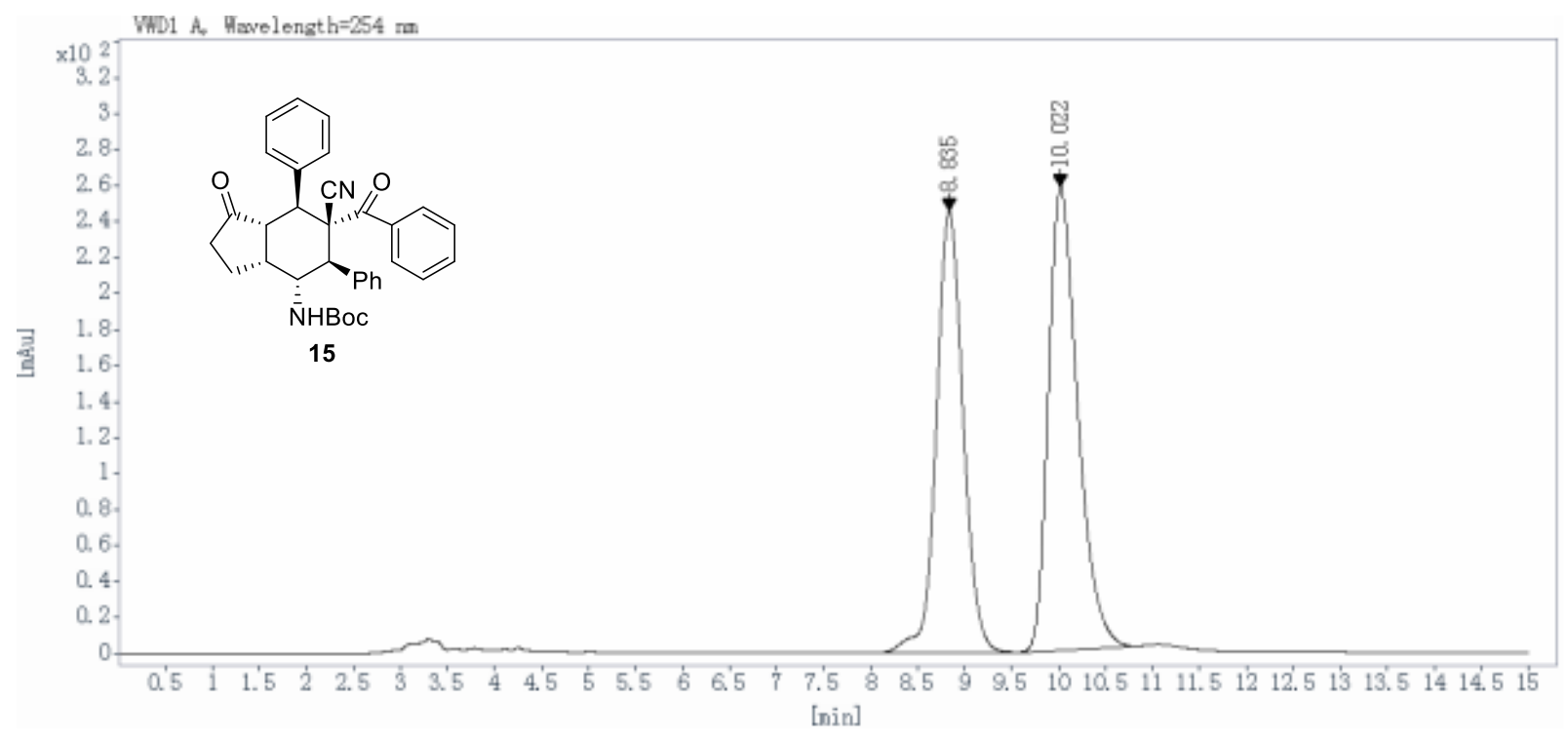

\begin{tabular}{|c|c|c|c|c|c|}
\hline $\begin{array}{l}\text { Ret Time } \\
\text { [min] }\end{array}$ & $\begin{array}{l}\text { Peak } \\
\text { Type }\end{array}$ & $\begin{array}{l}\text { Width } \\
\text { [min] }\end{array}$ & $\begin{array}{l}\text { Height } \\
\text { [mAU] }\end{array}$ & $\begin{array}{c}\text { Area } \\
{\left[\mathrm{mAU}^{* \mathrm{~s}]}\right.}\end{array}$ & $\begin{array}{c}\text { Area } \\
{[\%]}\end{array}$ \\
\hline 8.835 & BB & 0.31 & 244.9575 & 4879.1611 & 46.5816 \\
\hline 10.022 & BB & 0.33 & 257.0048 & 5595.2871 & 53.4184 \\
\hline
\end{tabular}

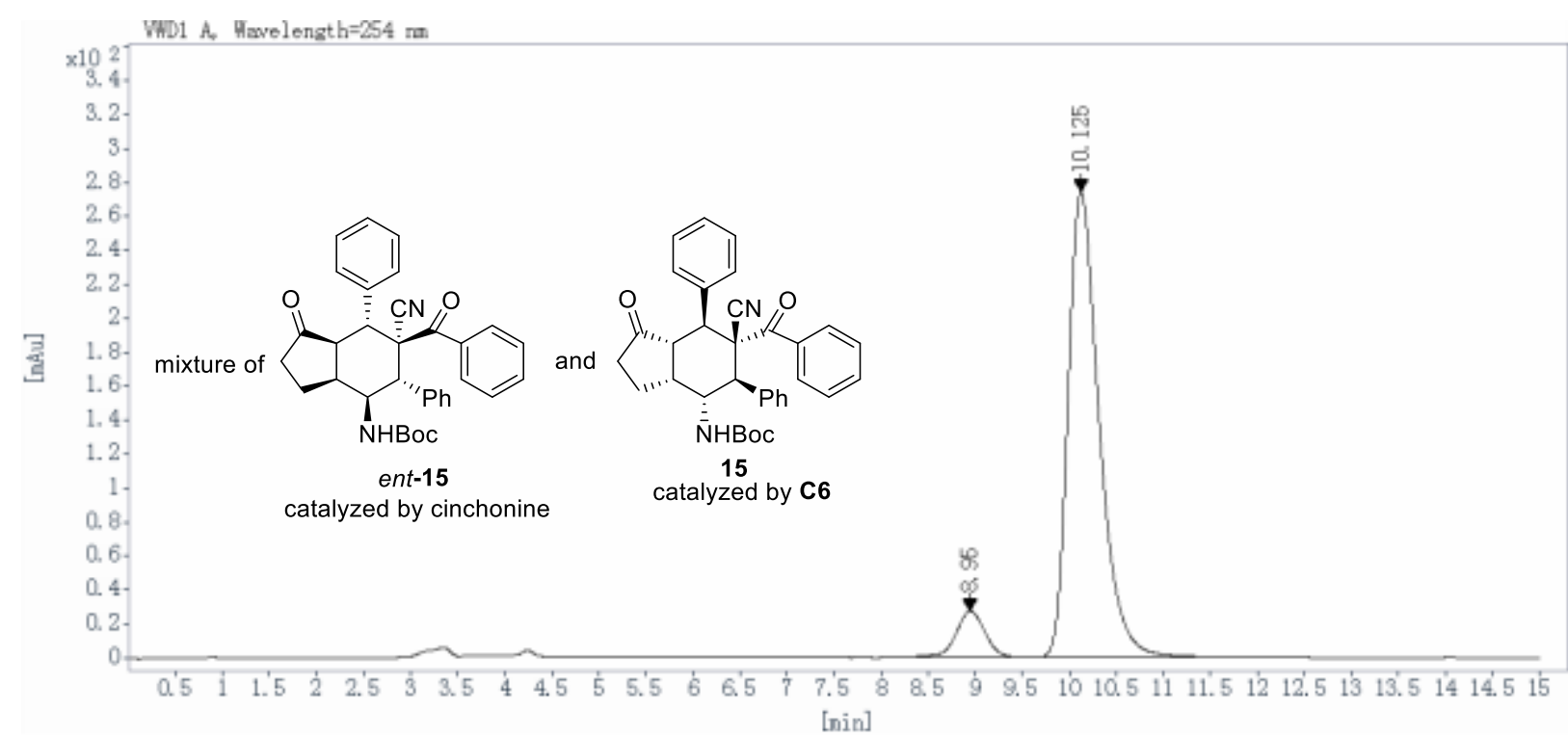

\begin{tabular}{cccccc}
$\begin{array}{c}\text { Ret Time } \\
\text { [min] }\end{array}$ & $\begin{array}{c}\text { Peak } \\
\text { Type }\end{array}$ & $\begin{array}{c}\text { Width } \\
{[\mathrm{min}]}\end{array}$ & $\begin{array}{c}\text { Height } \\
{[\mathrm{mAU}]}\end{array}$ & $\begin{array}{c}\text { Area } \\
{[\mathrm{mAU} \text { *s] }}\end{array}$ & $\begin{array}{c}\text { Area } \\
{[\%]}\end{array}$ \\
\hline 8.950 & BB & 0.33 & 27.2439 & 587.9487 & 8.5186 \\
10.125 & BB & 0.35 & $\begin{array}{c}273.8868 \\
\text { Totals: }\end{array}$ & 6313.9658 & 91.4814 \\
& & & 6901.9145 & 100.0000
\end{tabular}




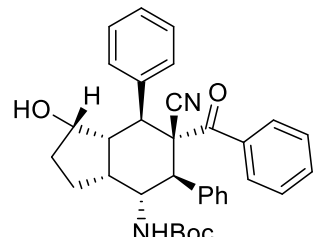

$16\left(100 \mathrm{MHz}, \mathrm{CDCl}_{3}\right)$
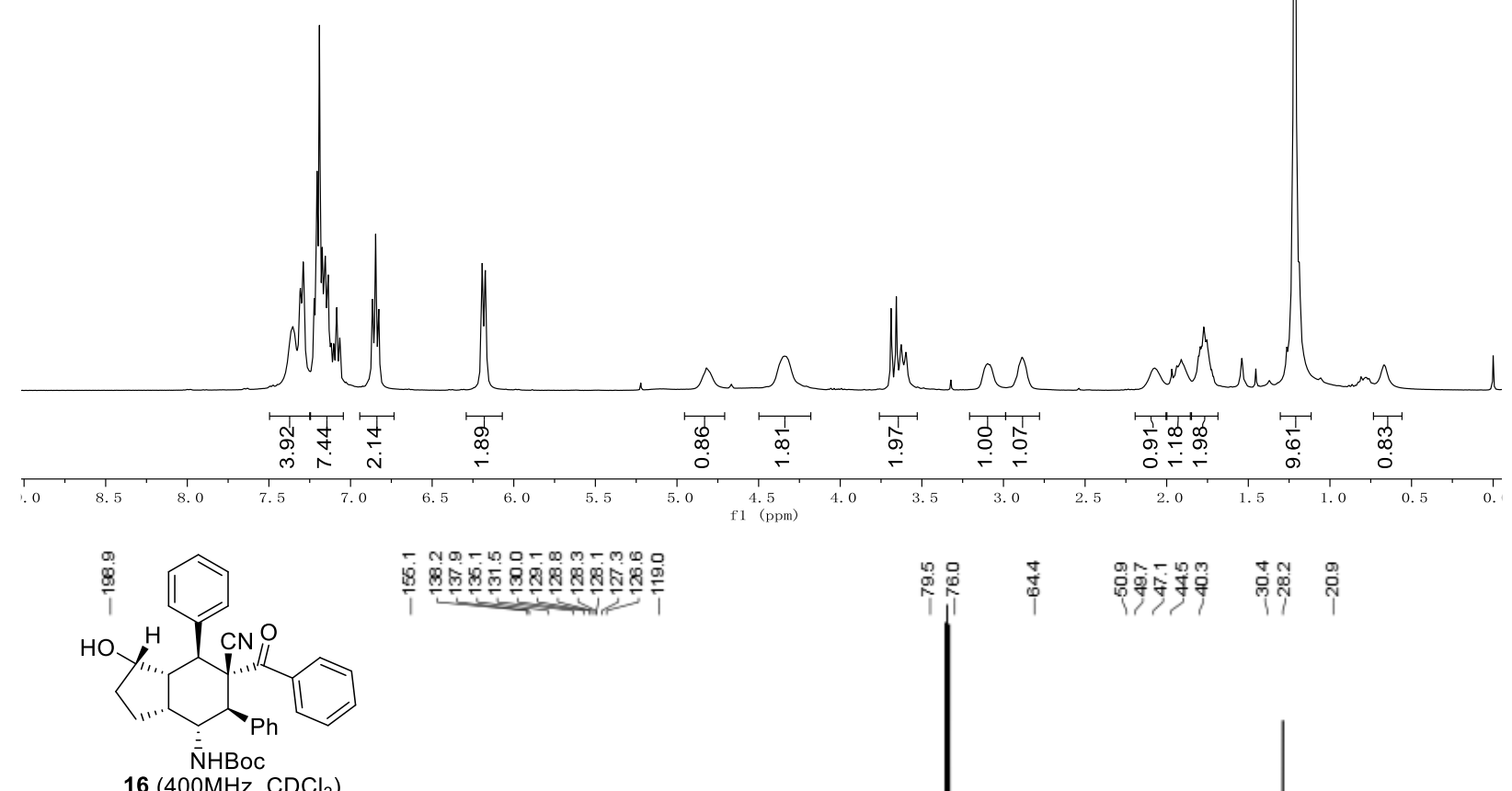

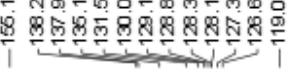

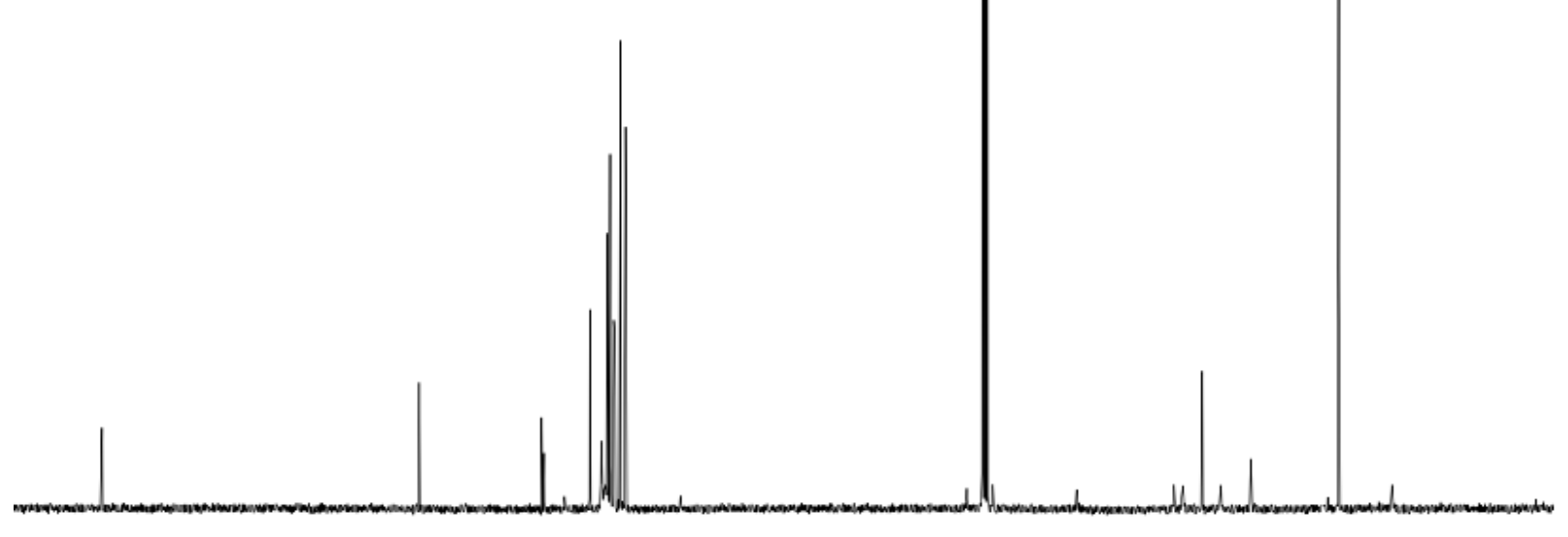

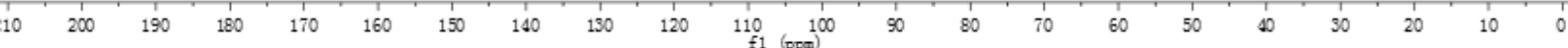




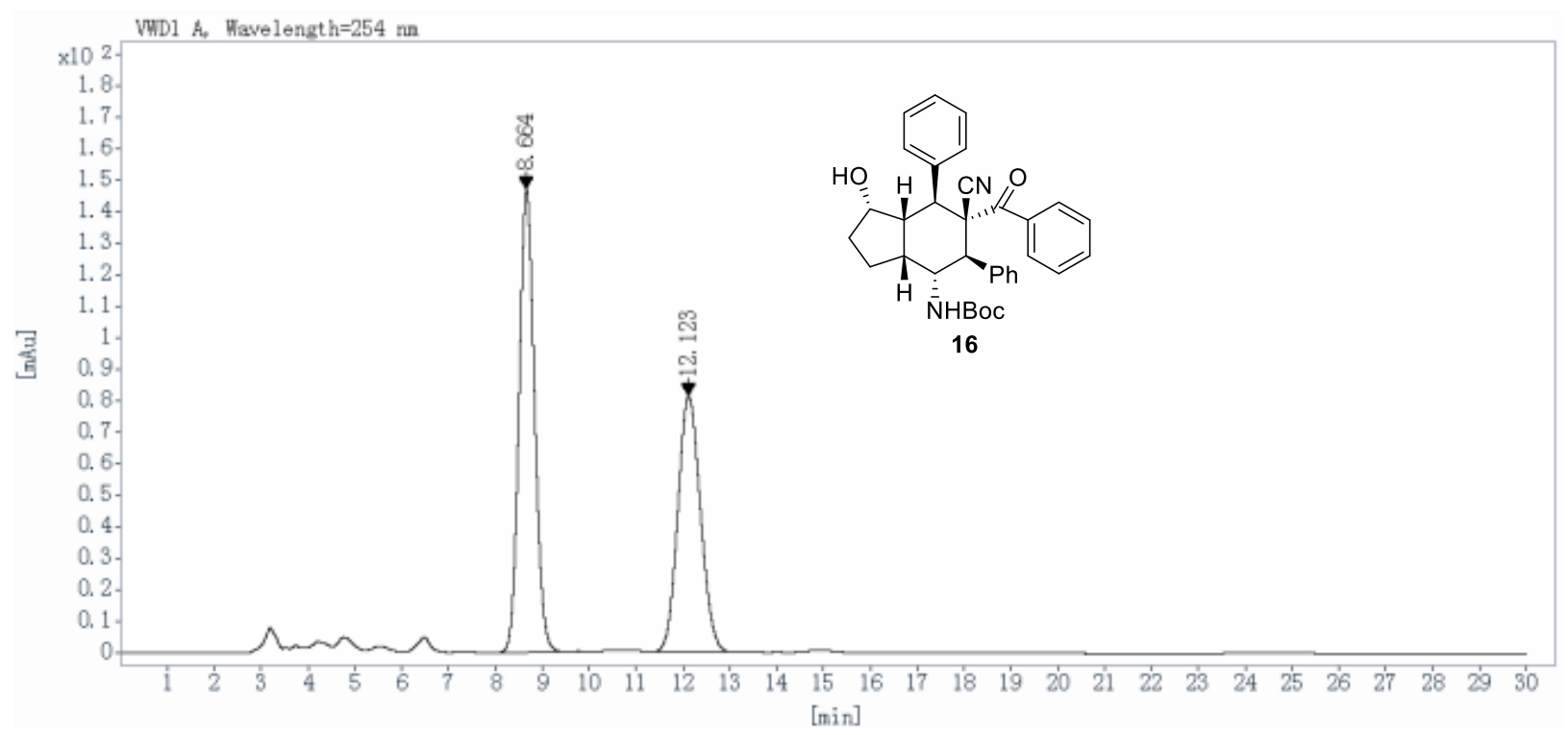

\begin{tabular}{cccccc}
$\begin{array}{c}\text { Ret Time } \\
\text { [min] }\end{array}$ & $\begin{array}{c}\text { Peak } \\
\text { Type }\end{array}$ & $\begin{array}{c}\text { Width } \\
{[\mathrm{min}]}\end{array}$ & $\begin{array}{c}\text { Height } \\
{[\mathrm{mAU}]}\end{array}$ & $\begin{array}{c}\text { Area } \\
{[\mathrm{mAU} \text { *s] }}\end{array}$ & $\begin{array}{c}\text { Area } \\
{[\%]}\end{array}$ \\
\hline 8.664 & BB & 0.36 & 146.8844 & 3369.2568 & 55.3245 \\
12.123 & BB & 0.52 & 81.1911 & 2720.7334 & 44.6755 \\
& & & Totals: & 6089.9902 & 100.0000
\end{tabular}

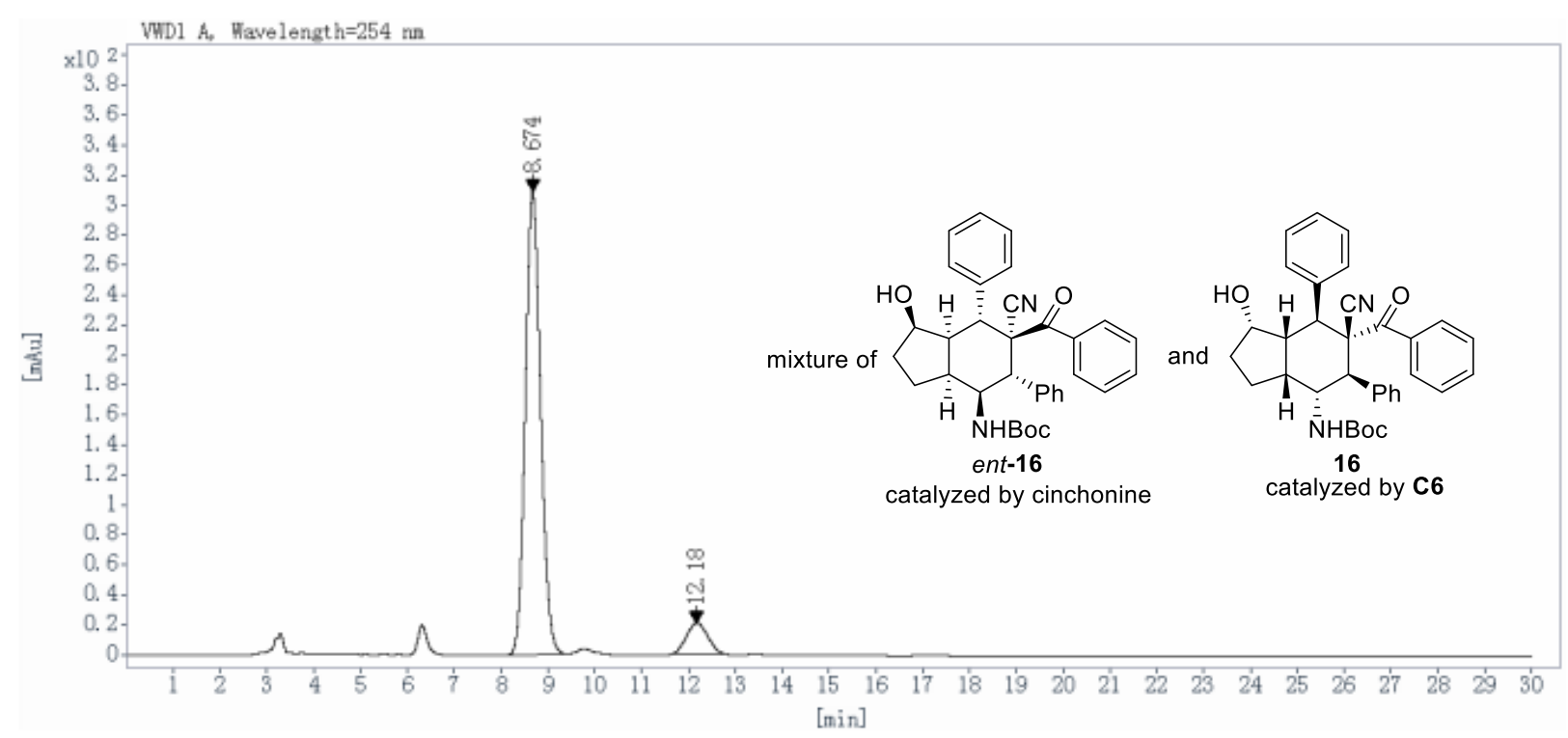

\begin{tabular}{|c|c|c|c|c|c|}
\hline $\begin{array}{l}\text { Ret Time } \\
\text { [min] }\end{array}$ & $\begin{array}{l}\text { Peak } \\
\text { Type }\end{array}$ & $\begin{array}{l}\text { Width } \\
\text { [min] }\end{array}$ & $\begin{array}{l}\text { Height } \\
{[\mathbf{m A U}]}\end{array}$ & $\begin{array}{c}\text { Area } \\
{\left[\mathrm{mAU}^{*} \mathbf{s}\right]}\end{array}$ & $\begin{array}{l}\text { Area } \\
{[\%]}\end{array}$ \\
\hline 8.674 & BB & 0.35 & 308.3178 & 7006.1206 & 91.4741 \\
\hline 12.180 & BBA & 0.50 & 20.6211 & 653.0064 & 8.5259 \\
\hline & & & Totals: & 7659.1270 & 100.0000 \\
\hline
\end{tabular}



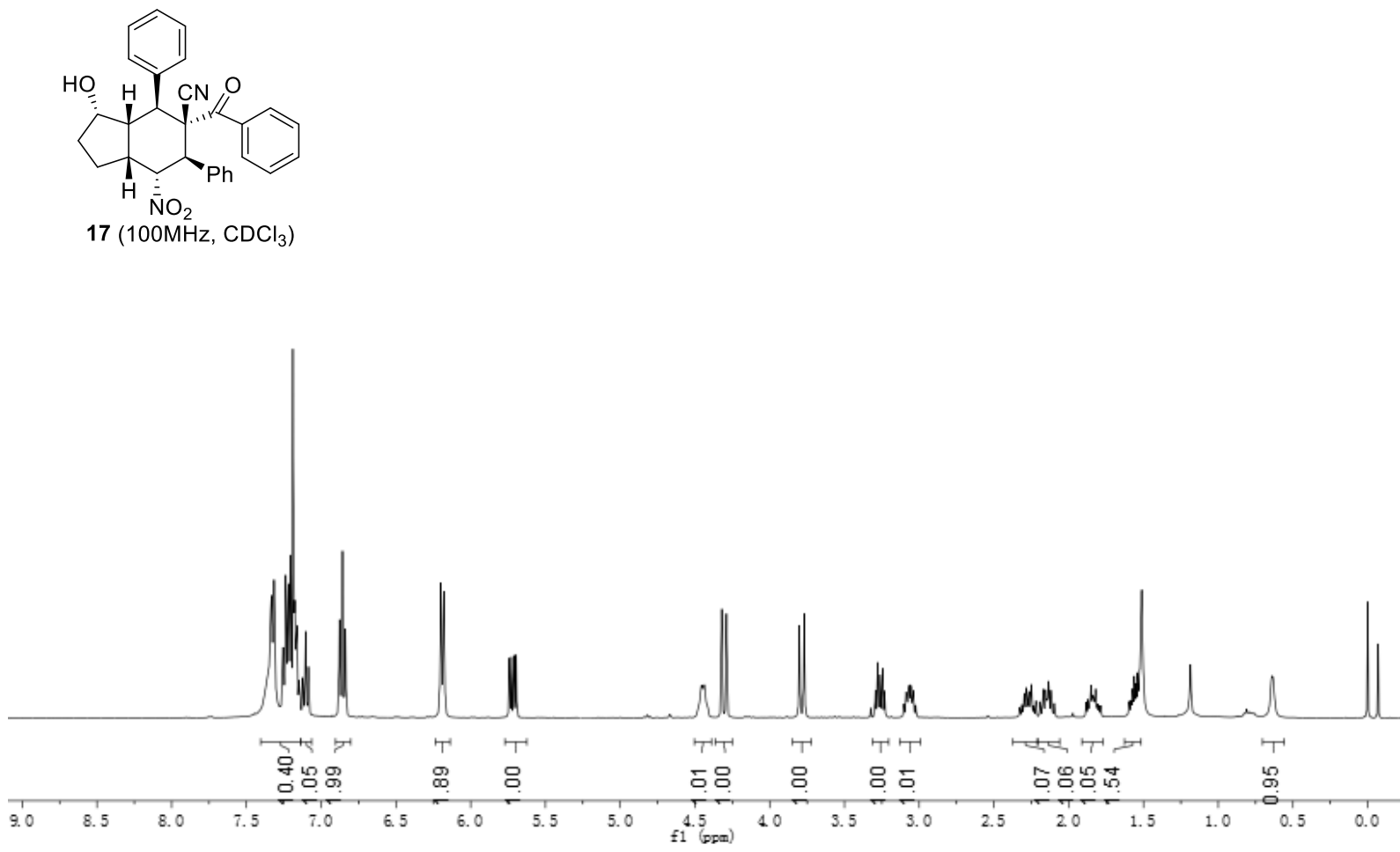

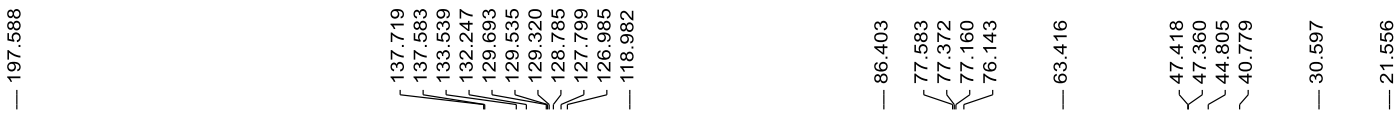

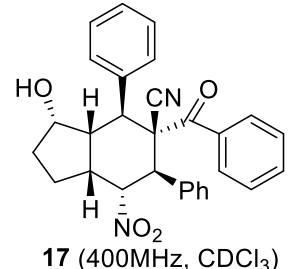

$17\left(400 \mathrm{MHz}, \mathrm{CDCl}_{3}\right)$

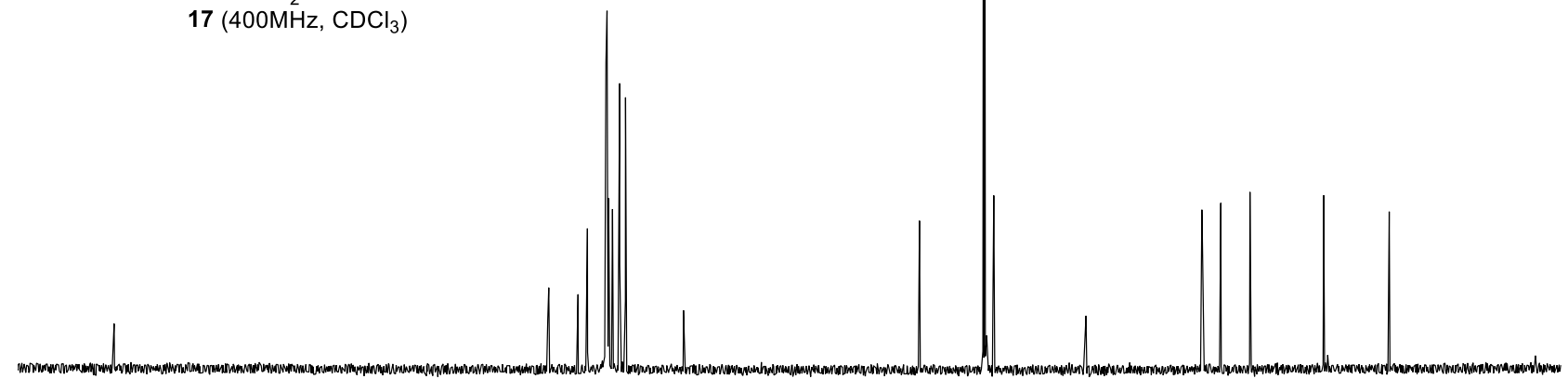

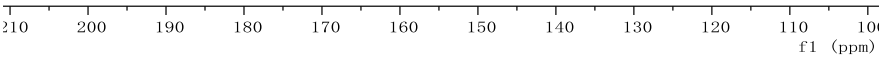




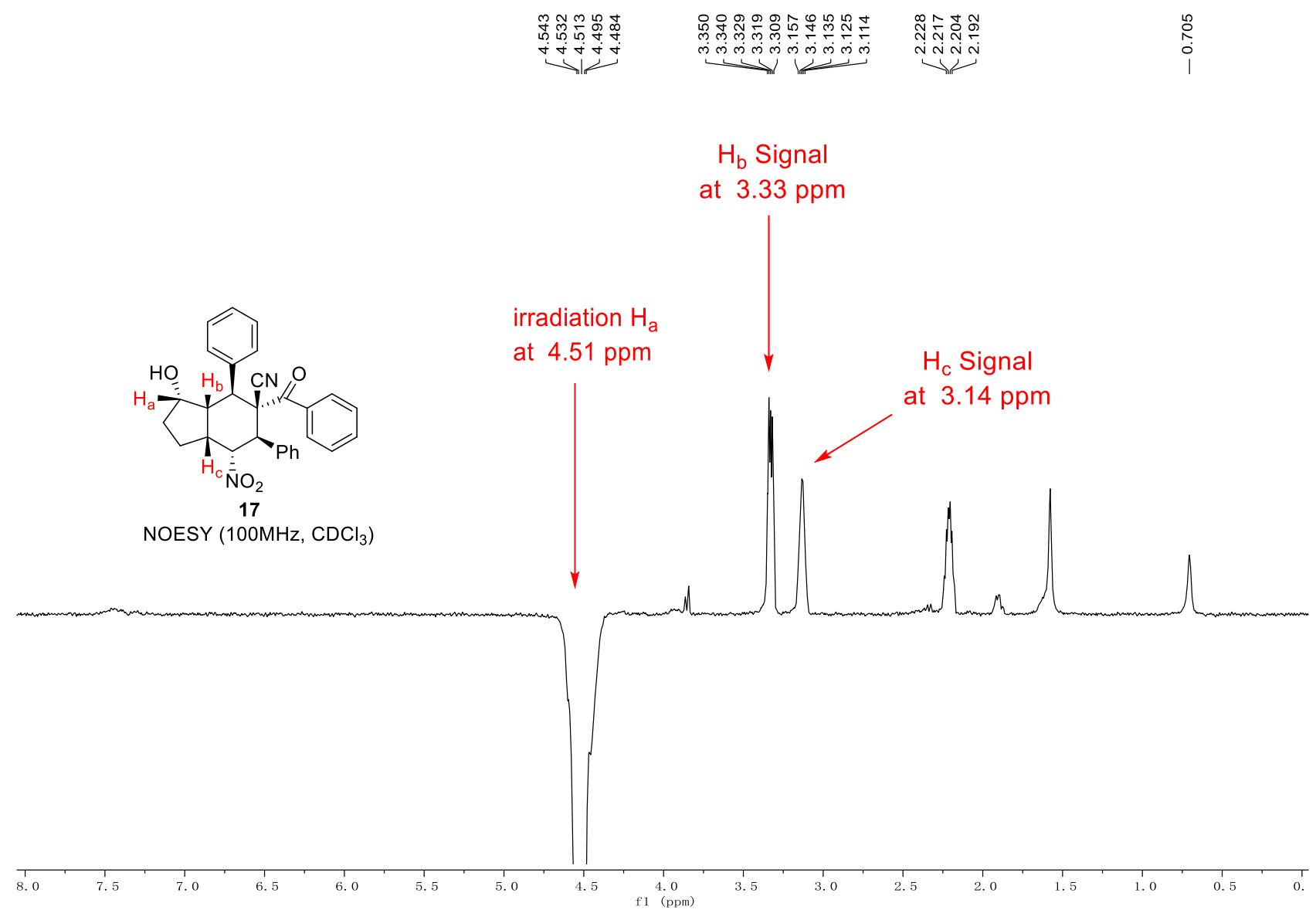




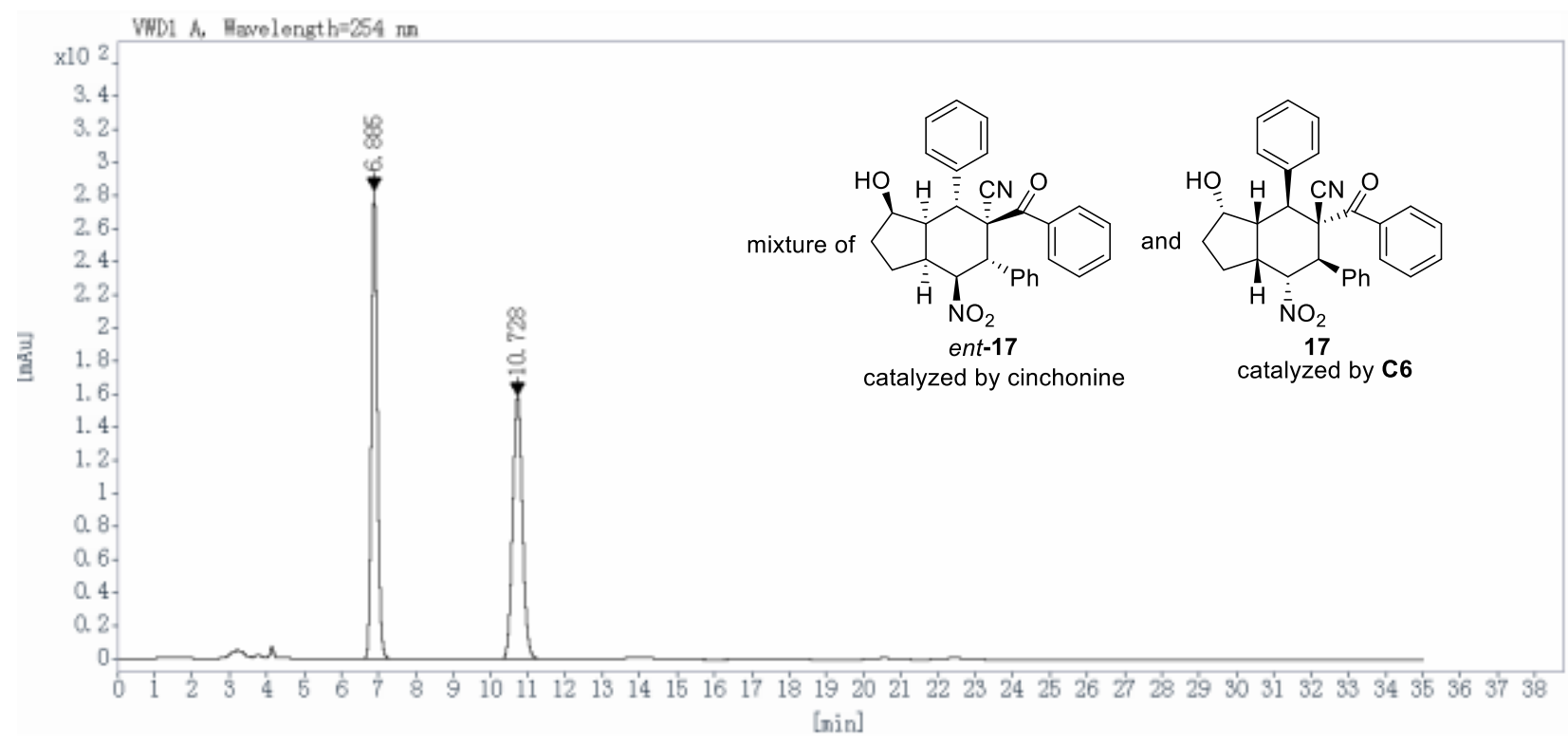

\begin{tabular}{|c|c|c|c|c|c|}
\hline $\begin{array}{l}\text { Ret Time } \\
\text { [min] }\end{array}$ & $\begin{array}{l}\text { Peak } \\
\text { Type }\end{array}$ & $\begin{array}{l}\text { Width } \\
\text { [min] }\end{array}$ & $\begin{array}{l}\text { Height } \\
\text { [mAU] }\end{array}$ & $\begin{array}{c}\text { Area } \\
{\left[\mathbf{m A U} \mathbf{U}^{*} \mathbf{s}\right]}\end{array}$ & $\begin{array}{l}\text { Area } \\
{[\%]}\end{array}$ \\
\hline 6.885 & BB & 0.17 & 282.8138 & 3109.9202 & 51.8464 \\
\hline 10.728 & VB R & 0.28 & 159.7134 & 2888.4150 & 48.1536 \\
\hline & & & Totals: & 5998.3352 & 100.0000 \\
\hline
\end{tabular}

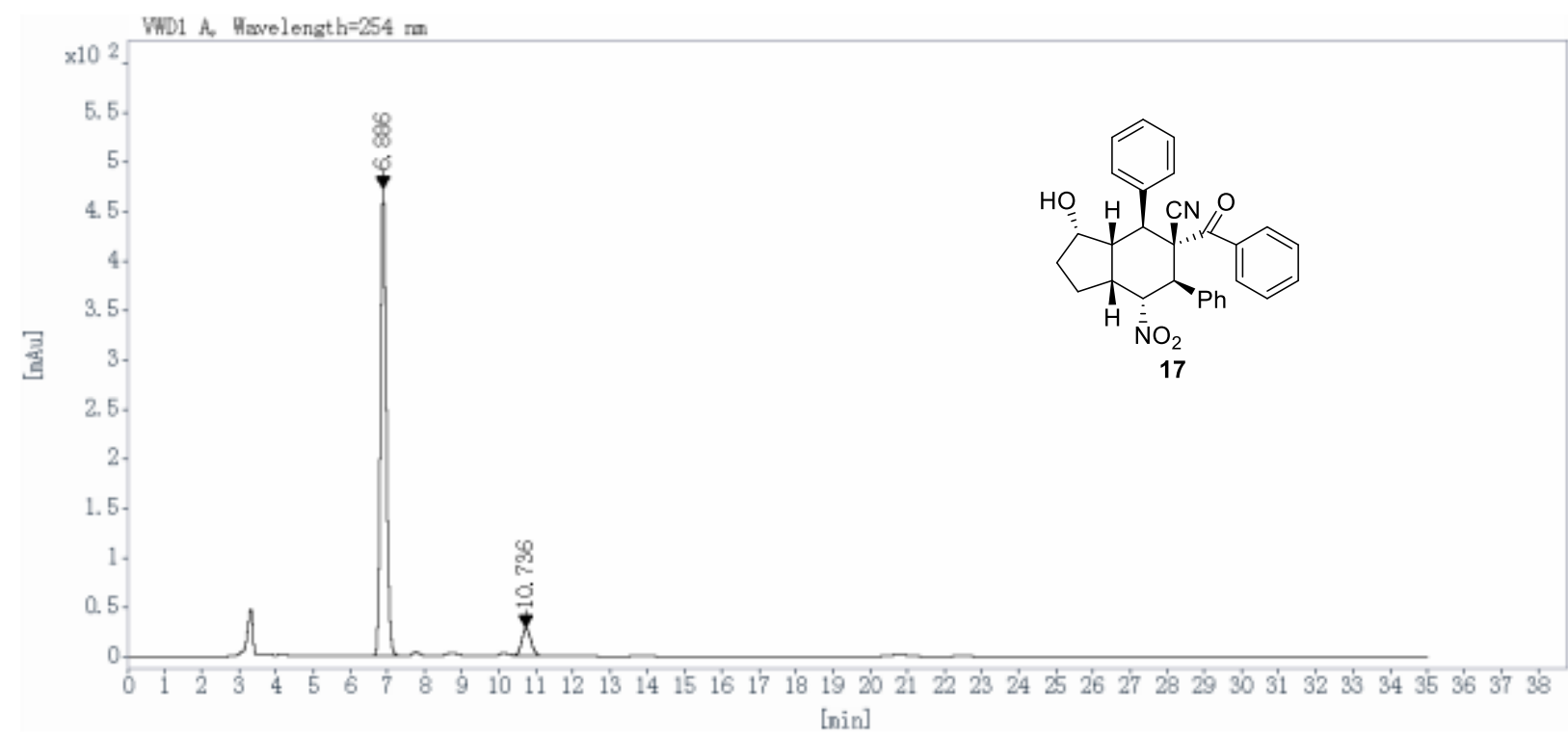

\begin{tabular}{cccccc}
$\begin{array}{c}\text { Ret Time } \\
{[\mathrm{min}]}\end{array}$ & $\begin{array}{c}\text { Peak } \\
\text { Type }\end{array}$ & $\begin{array}{c}\text { Width } \\
{[\mathrm{min}]}\end{array}$ & $\begin{array}{c}\text { Height } \\
{[\mathrm{mAU} \text { ] }}\end{array}$ & $\begin{array}{c}\text { Area } \\
{[\mathrm{mAU} \text { *s] }}\end{array}$ & $\begin{array}{c}\text { Area } \\
{[\%]}\end{array}$ \\
\hline 6.886 & VB R & 0.17 & 471.7472 & 5078.5771 & 91.3756 \\
10.736 & BB & 0.27 & 27.4894 & 479.3391 & 8.6244 \\
& & & Totals: & 5557.9163 & 100.0000
\end{tabular}




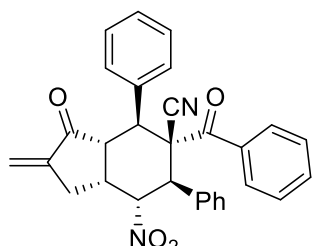

$18\left(400 \mathrm{MHz}, \mathrm{CD}_{3} \mathrm{OD}\right)$

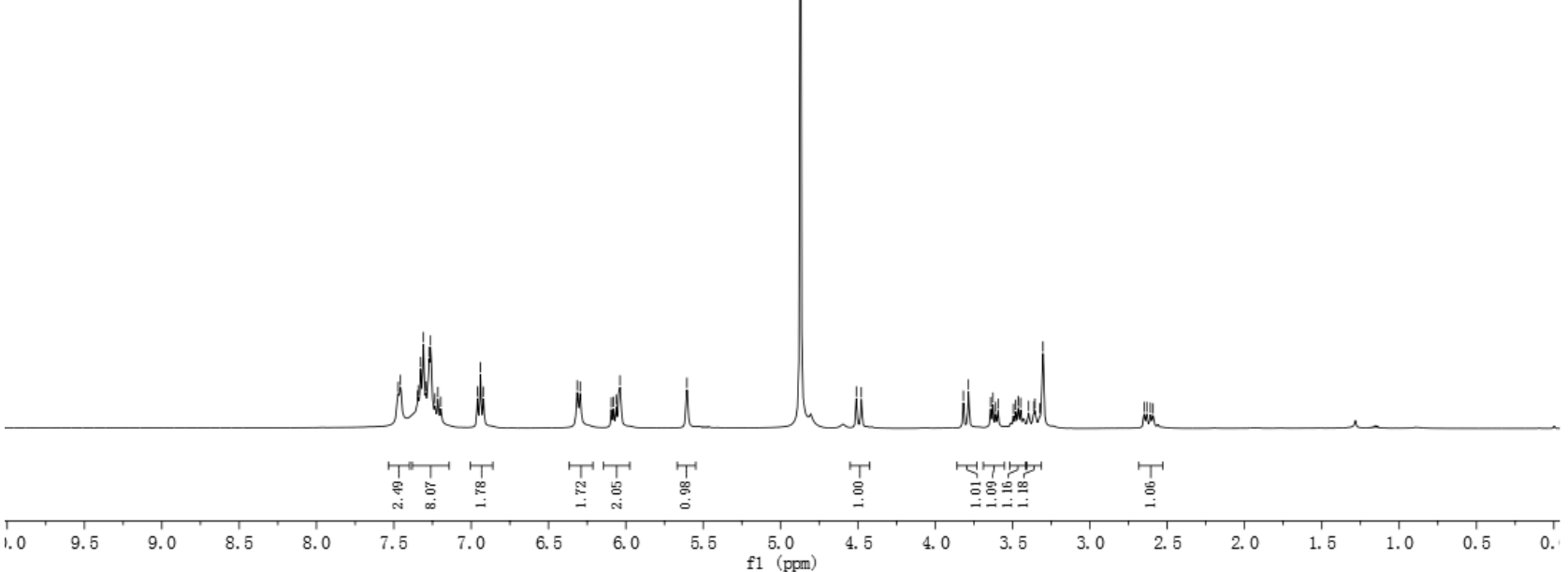

筫

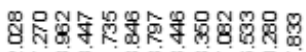
은

\section{$\underset{\frac{8}{6}}{\frac{8}{\infty}}$}

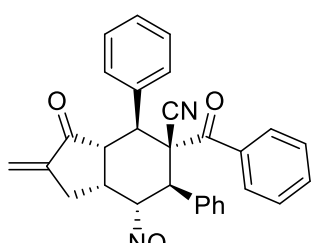

$18\left(100 \mathrm{MHz}, \mathrm{CD}_{3} \mathrm{OD}\right)$

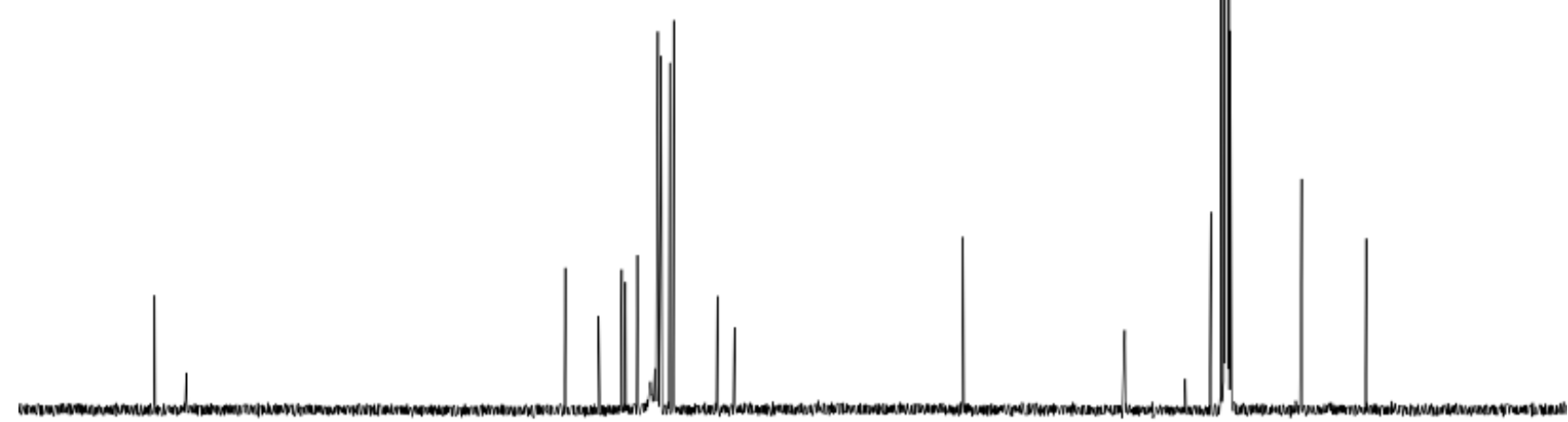

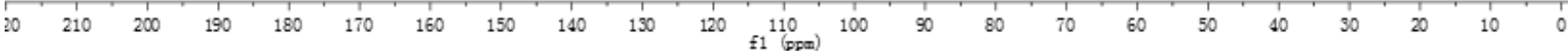




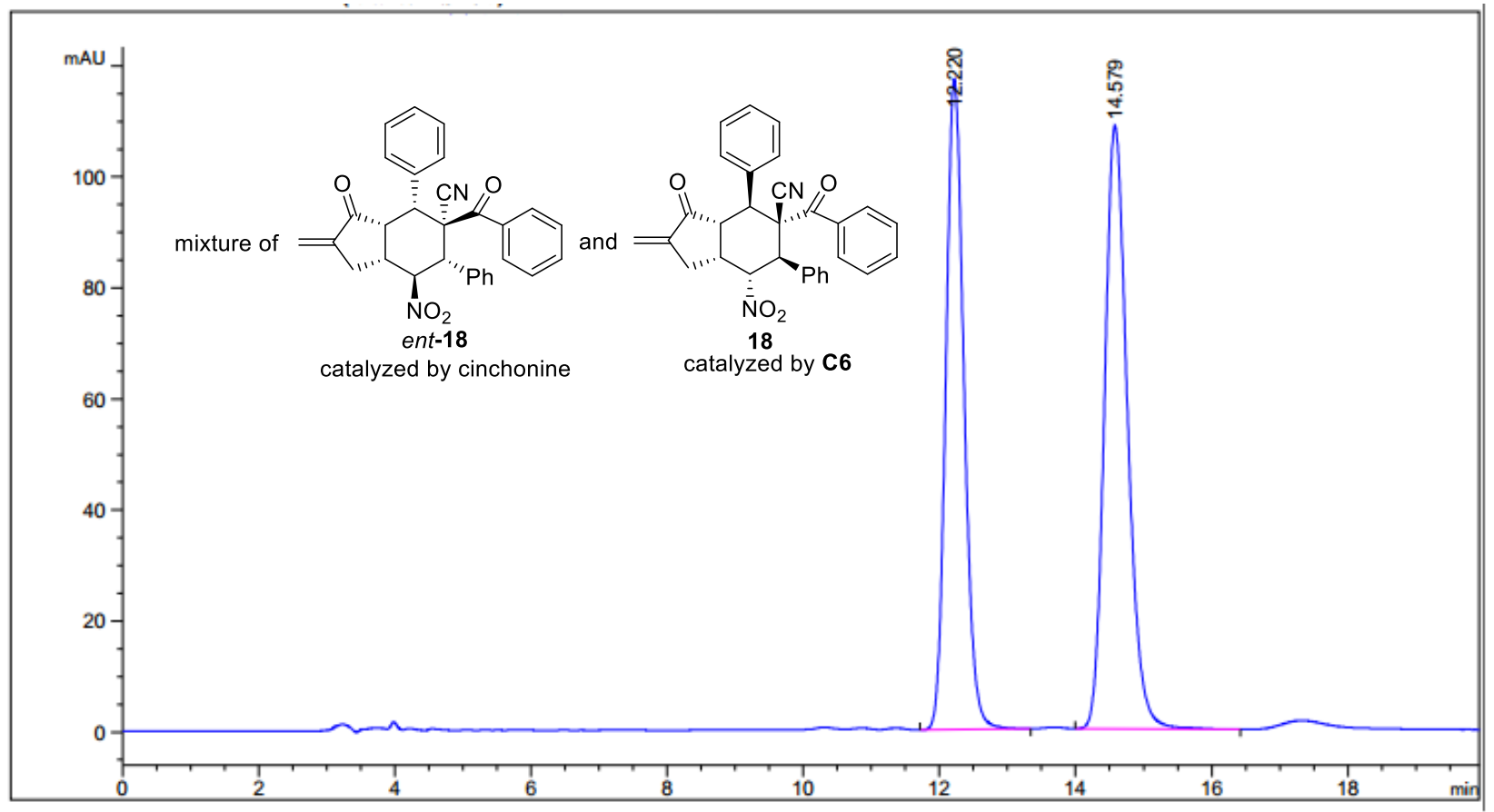

Signal 1: VWD1 A, Wavelength=254 nm
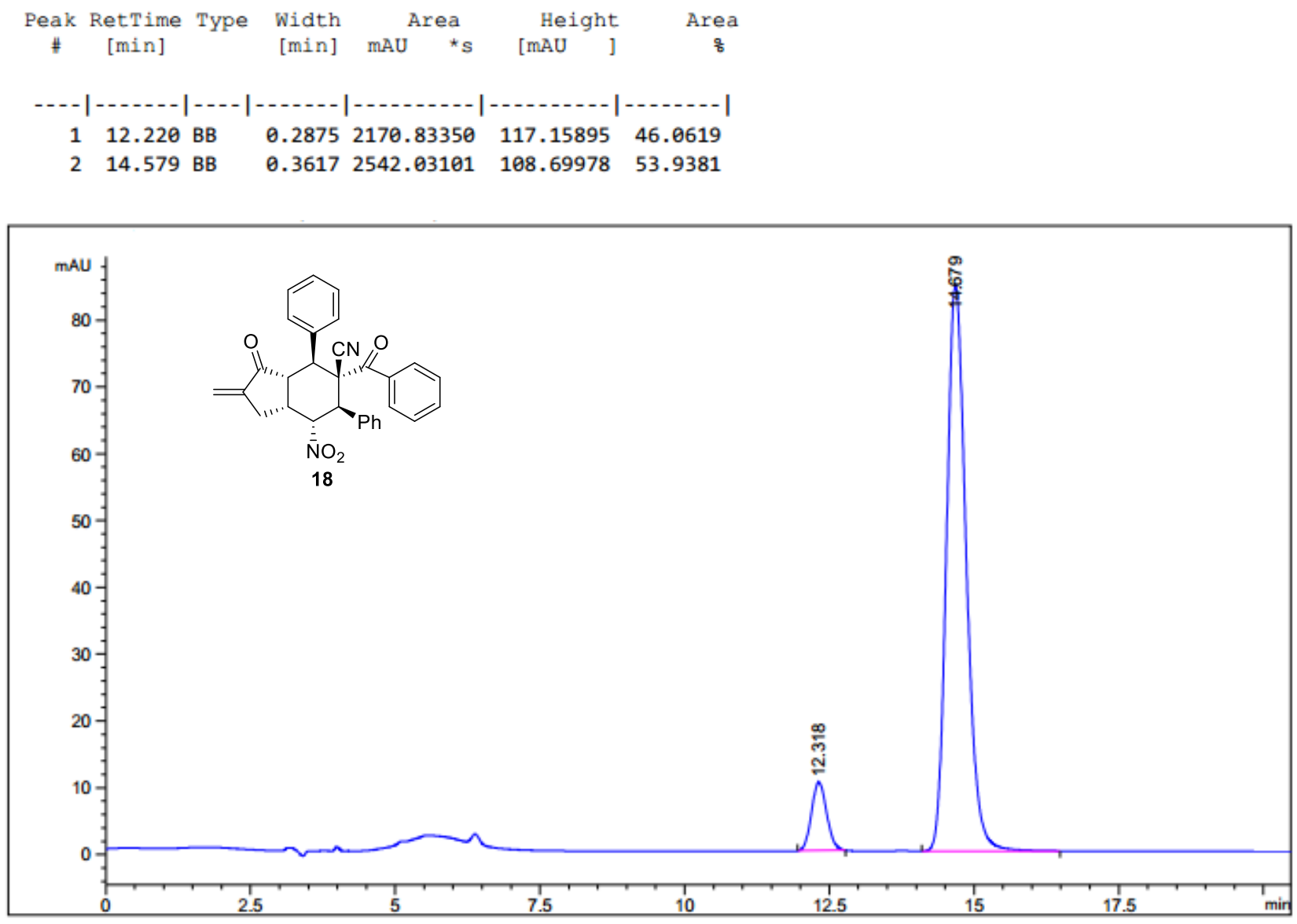

Signal 1: VWD1 A, Wavelength=254 nm

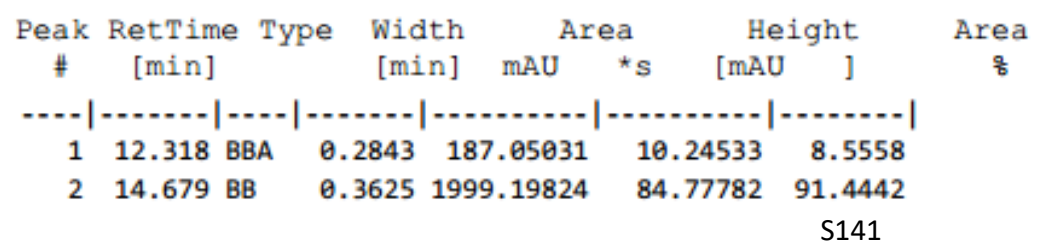

